\title{
PSEUDO INVERSE FILTER DESIGN FOR IMPROVING THE AXIAL RESOLUTION OF ULTRASOUND IMAGES
}

\author{
Warren Frazer Young
}

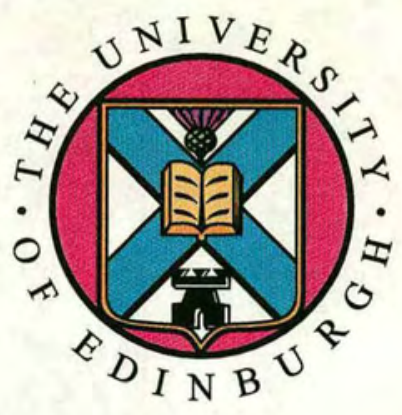

A thesis submitted for the degree of Doctor of Philosophy.

August 1998

The University of Edinburgh,

Department of Electrical Engineering,

King's Buildings,

Mayfield Road,

Edinburgh EH9 3JL 


\section{Abstract}

This thesis is concerned with improving the quality of images from medical ultrasound examinations which often suffer from poor picture quality. In addition, the resolution of ultrasound images is poor compared to other medical imaging techniques. Ultrasound images have comparatively poor resolution (axial, lateral, and contrast), and poor dynamic range.

The axial resolution, and contrast resolution of medical ultrasound images can be improved by appropriate filtering of the RF ultrasound signal, prior to signal demodulation. In this thesis a pseudo inverse filter is presented, suitable for improving the axial and contrast resolution of medical ultrasound images. This filter is based on a sigmoid function, and it approximates an inverse filter - but is less sensitive to noise than an inverse filter.

The filter is designed to provide a trade-off between a matched filter and an inverse filter, by appropriate selection of design parameters. The matched filter is theoretically optimal for signal to noise ratio for a stationary process in the presence of additive white Gaussian noise (AWGN), and the inverse filter is theoretically optimal for image fidelity or resolution for a stationary process in the absence of noise. However, the inverse filter will also amplify any noise in the image, and the matched filter degrades the image resolution. The sigmoid based pseudo inverse filter trades off the characteristics of the matched and inverse filters in that it retains some resolution enhancement, while not allowing the noise to be unduly amplified.

Results from applying the proposed filter are presented, with a variety of parameter settings, to ultrasound images of three target objects: a near perfect plane reflector; a tissue mimicking phantom; and intravascular ultrasound (IVUS) images from the aorta of a rabbit. These three targets provide good test cases for the filter: the near 
perfect plane reflector is a good test of the filter under (near) ideal circumstances; the IVUS images are a realistic test of a medical situation in that they are virtually identical to human IVUS images; and the phantom provides a good midpoint between the first two cases. This midpoint is useful since it allows me to analyse a realistic image, while knowing exactly what the structure and shape of the target object is - this knowledge is not available for the IVUS images.

Lastly, extensive comparisons with other filters are presented that have been proposed for the filtering of ultrasound. These comparisons are both subjective and more importantly - objective. In particular comparisons are made with the following filters: matched, inverse, phase correction, square root based, fourth root based, and Wiener filters. 


\section{ACKNOWLEDGEMENTS}

I wish to acknowledge the assistance given to me by the following people:

Dr. Tim Spencer, and Mr Calum McLean, for providing me with IVUS data, and for useful discussions. Mr. Tom Anderson, and also Lynn Fenn for their assistance in providing me with phantom ultrasound data.

Mr Nick Palmer, Dr. Ken Boddy, and Prof. Norman McDicken (my second supervisor) for useful discussions from a medical perspective.

Dr. Bill Nailon, for introducing me to IVUS, and for his encouragement and many useful discussions.

Angus Condy for his assistance in proof reading this work.

My third supervisor Dr Jimmy Dripps for his many suggestions, and my first supervisor Dr. Steve McLaughlin for the many hours given to this project over three years.

I wish to acknowledge financial assistance I have received from the British Governments ORS award scheme, a University of Edinburgh Postgraduate Studentship, and from my parents, Ken and Carole Young.

I wish to thank my parents and other family members for their continual support and encouragement throughout the time of this work.

Finally, I wish to thank Megan, my wife who has supported me fully throughout this project, and has given birth to Nathan, my first child - whose first recorded likeness can be seen in Figure 1.1, on page 2, and my yet to be born second child whose likeness can be seen in Figure 2.2 on page 12. Thank you Megan for your love and support.

This thesis is dedicated to the glory of God. 


\section{CONTENTS}

AbSTraCT

I

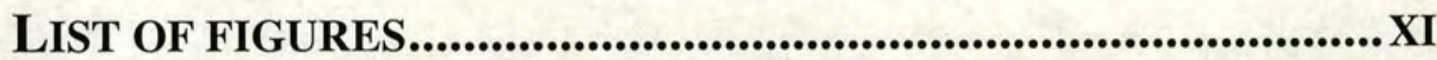

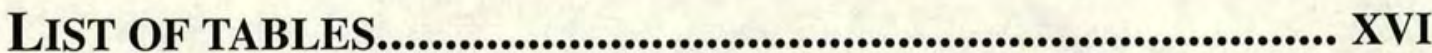

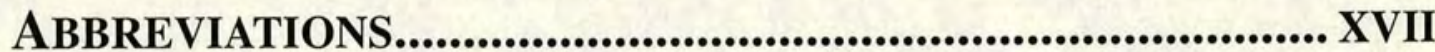

LIST OF PRINCIPAL SYMBOLS .......................................XVIII

MEDICAL TERMINOLOGY ............................................... XIX

Chapter 1. InTROdUCTION ............................................... 1

1.1. Introduction ..................................................................................................1

1.2. Medical ultrasound .................................................................................2

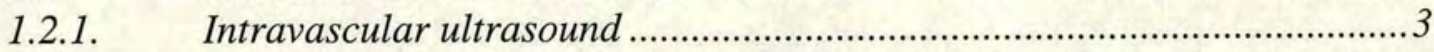

1.3. Ultrasound processing ..............................................................................

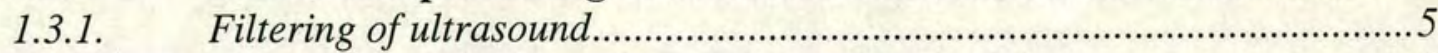

1.3.2. Three dimensional imaging of ultrasound …………………………......

1.4. Organisation of the thesis............................................................................

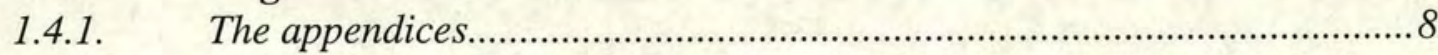

CHAPTER 2. Ultrasound IMAGING ................................. 10

2.1. Introduction..................................................................................................10 


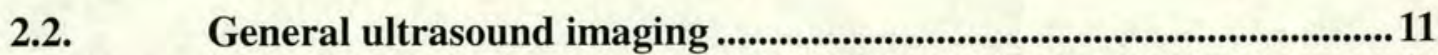

2.2.1. Required system components ............................................................... 13

2.2.2. Differences in medical ultrasound imaging systems...............................13

2.3. Reflection and transmission based ultrasound ....................................14

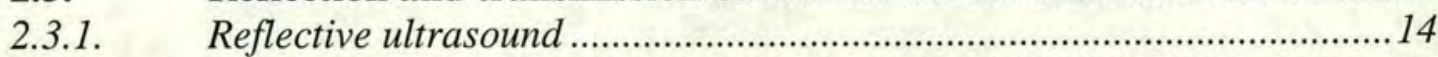

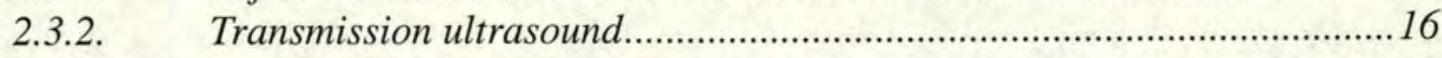

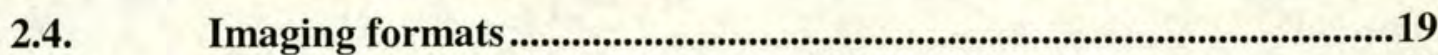

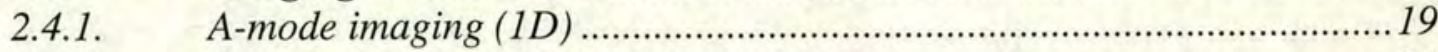

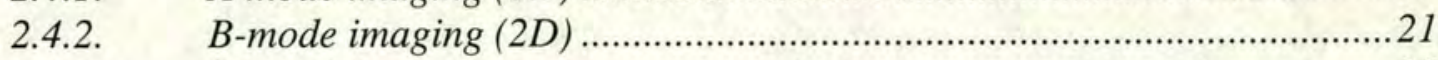

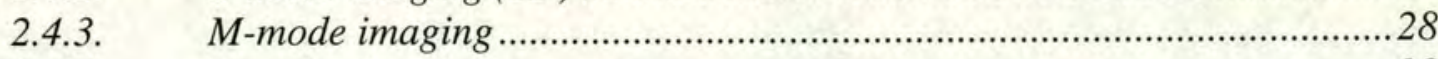

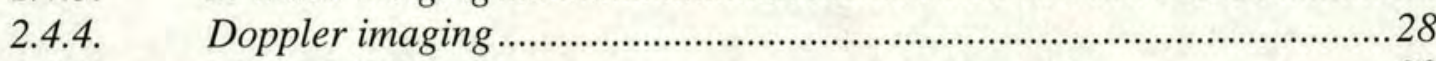

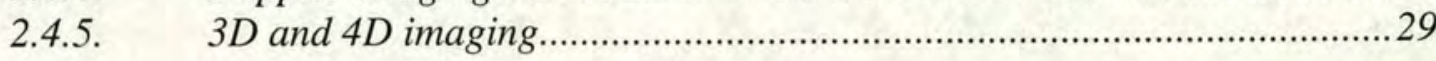

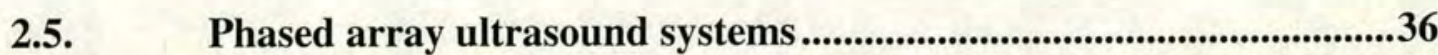

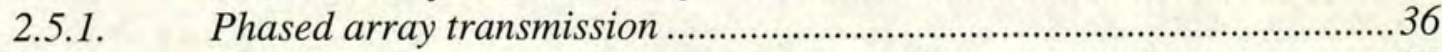

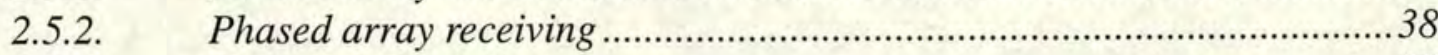

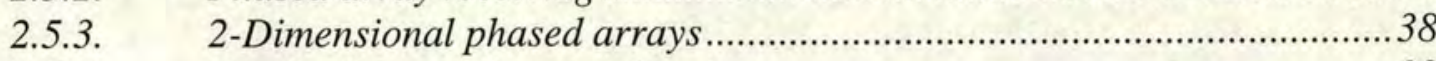

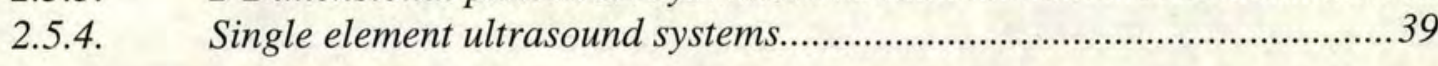

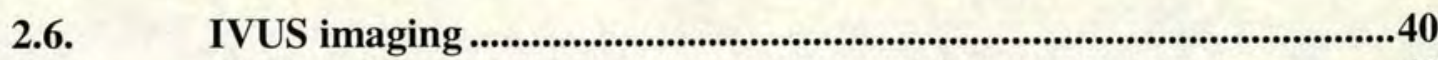

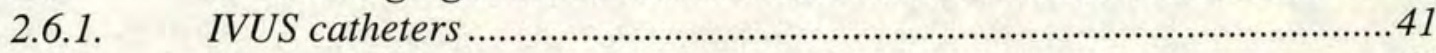

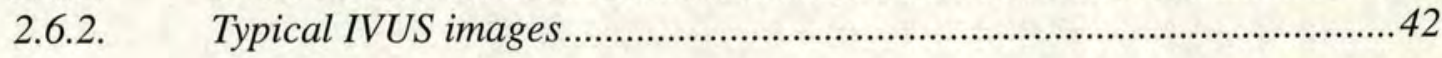

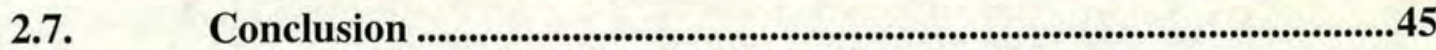

CHAPTER 3. FILTERING ….............................................................. 46

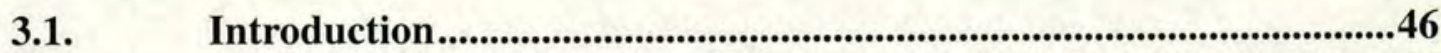

3.1.1. The assumed model for filter design ...................................................... 48

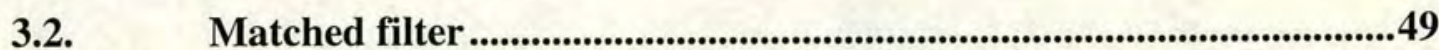

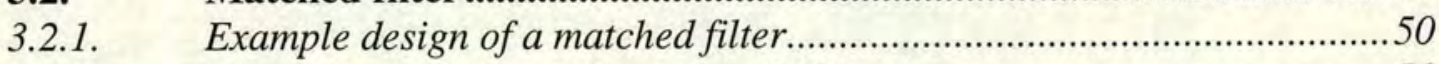

3.2.2. Example application of a matched filter ................................................51

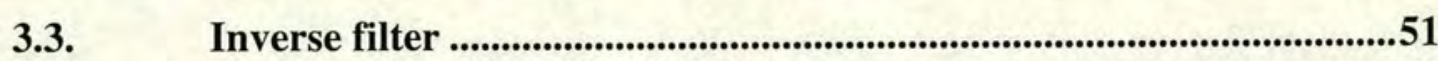

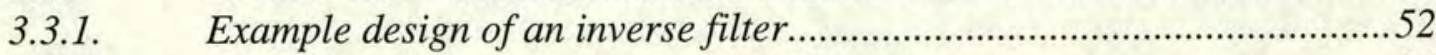

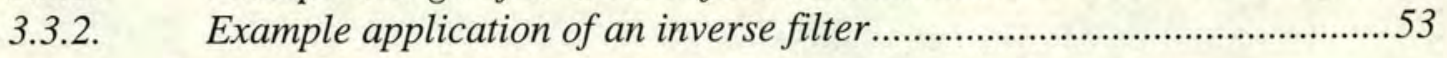

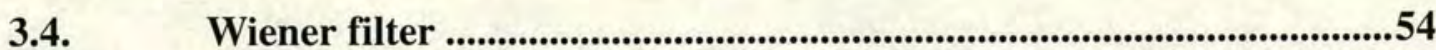

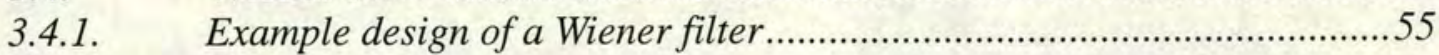

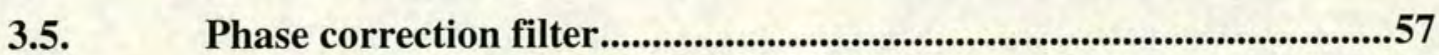

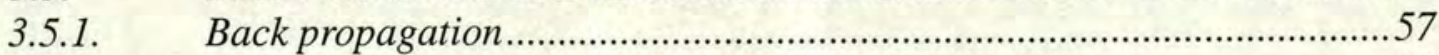

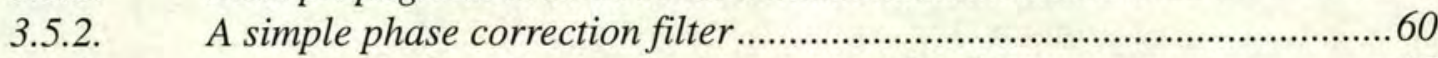

3.5.3. Example design of a simple phase correction filter ................................60

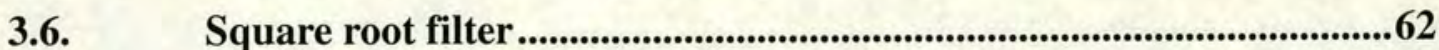

3.6.1. Example design of a square root filter ....................................................63

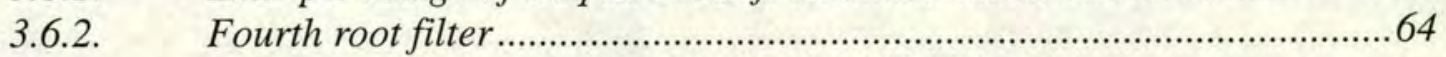


3.6.3. The matched, phase correction, and inverse filters as $n^{\text {th }}$ root filters......65

3.7. Other attempts at pseudo inverse filtering of ultrasound ....................66

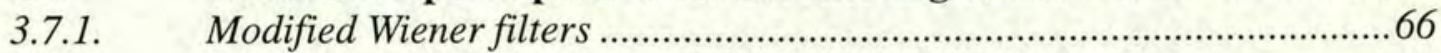

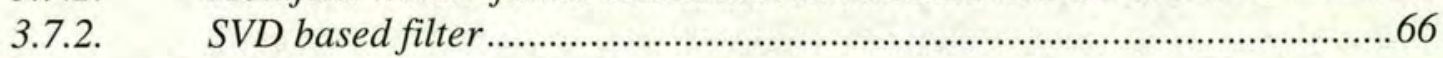

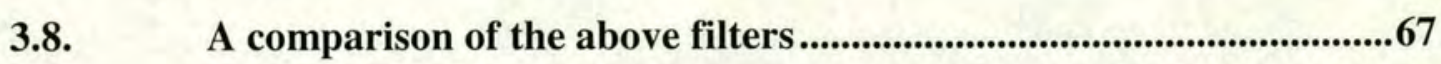

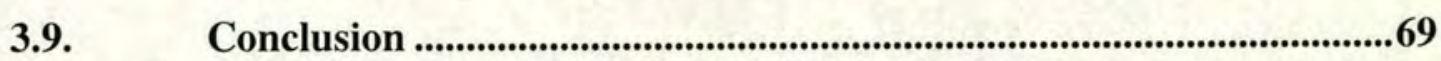

\section{CHAPTER 4. SIGMOID BASED PSEUDO INVERSE FILTER}

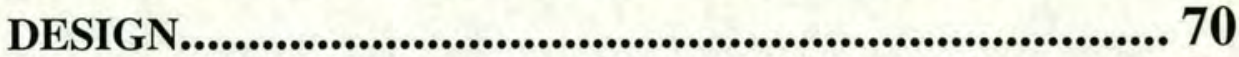

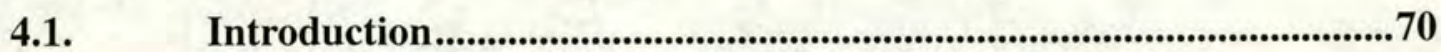

4.2. Background model assumptions ...............................................................70

4.2.1. Measuring the transducer's impulse response ........................................73

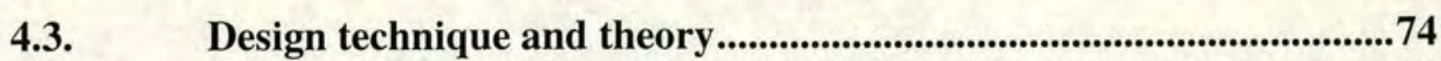

4.3.1. Modifications from previously published specification ..........................77

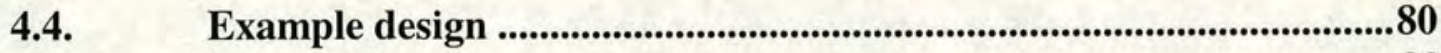

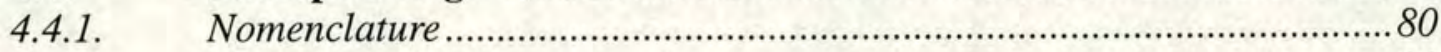

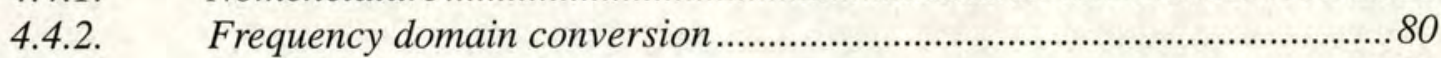

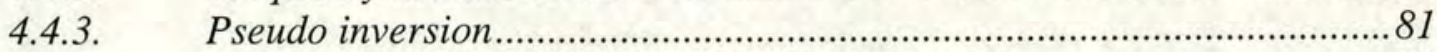

4.4.4. Conversion back to time domain - making the filter taps ........................83

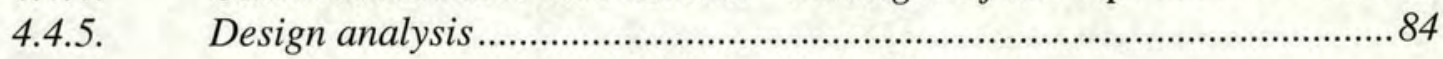

4.5. Windowing effects on filter taps ..........................................................86

4.6. Comparison with other filters................................................................88

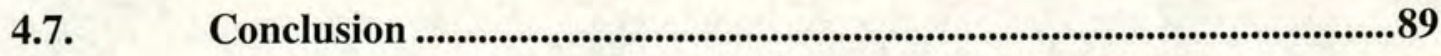

Chapter 5. Ultrasound SETUP ..................................... 91

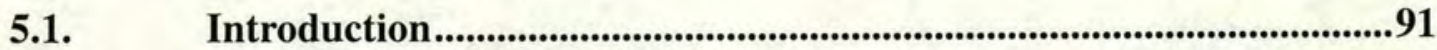

5.2. IVUS data collection system...................................................................91

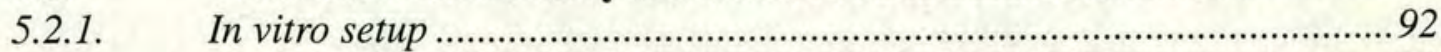

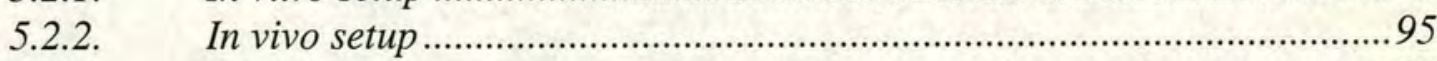

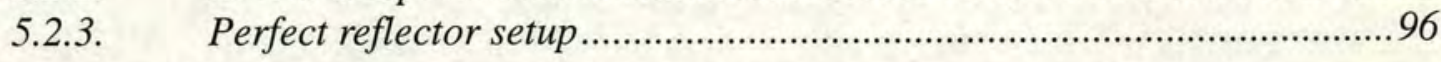

5.3. Phased array (phantom) data collection ................................................97

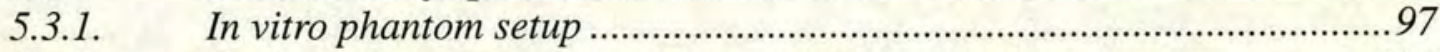

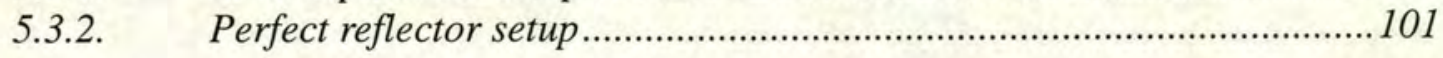

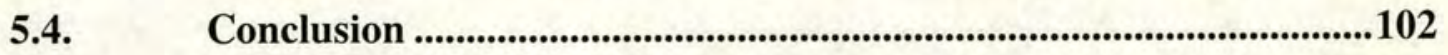


CHAPTER 6. RESUlTS OF PSEUDO INVERSE FILTERS ON ULTRASOUND NEAR PERFECT REFLECTIONS.......... 103

6.1. Introduction .............................................................................................103

6.2. Comparison of filtered near perfect reflections with different parameter values.....................................................................................105

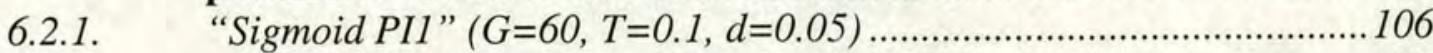

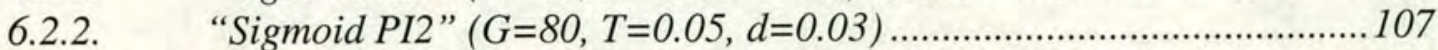

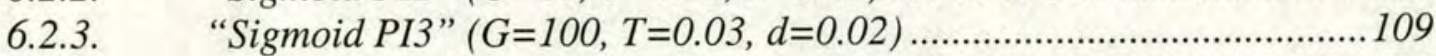

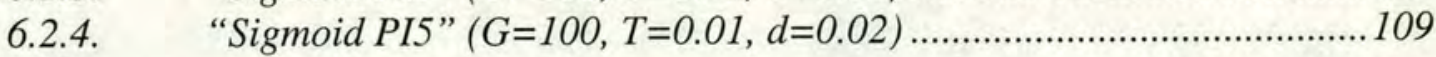

6.3. Comparison with other filters...............................................................110

6.3.1. Comparison with Wiener filters ………...........................................113

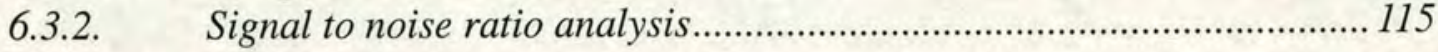

6.4. Conclusion ................................................................................................... 117

CHAPTER 7. RESULTS ON PHANTOM IMAGES ........................118

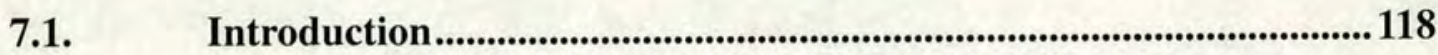

7.2. Application of P.I. filters to phased arrays ..........................................118

7.2.1. Phased array transducer characteristics...............................................119

7.2.2. Phased array variance with axial distance ………………………….....122

7.3. Image reconstruction procedure............................................................123

7.3.1. The example image used …………………......................................123

7.4. Images filtered with sigmoid based PI filters .....................................125

7.4.1. Analysis of main lobe width vs. side lobe suppression ……...................127

7.5. Second analysis with neutral phase.....................................................129

7.5.1. The problem with signal phase from a phased array.............................129

7.5.2. A possible solution ...............................................................................130

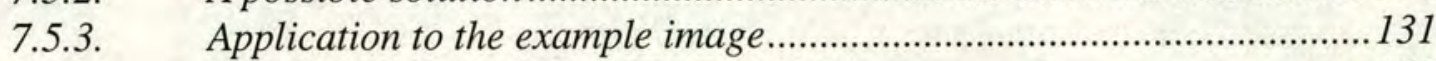

7.5.4. Analysis of main lobe width vs. side lobe suppression ……....................133

7.6. Conclusion ..............................................................................................135

\section{CHAPTER 8. RESUlTS OF PSEUDO INVERSE FILTERS ON} IVUS ULTRASOUND IMAGES.................................. 136

8.1. Introduction ...........................................................................................136

8.2. Image reconstruction procedure..........................................................136

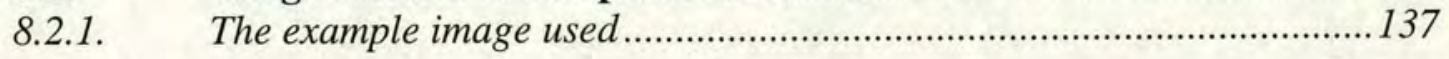

8.3. Regions and lines of interest.................................................................139 
8.4. Images filtered with sigmoid based PI filters ....................................139

8.5. Images filtered with other filters ..........................................................144

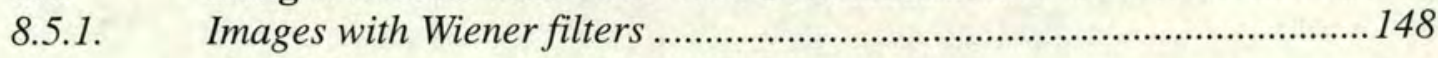

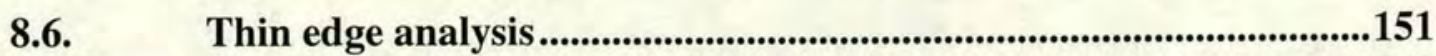

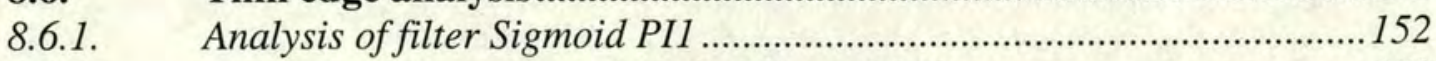

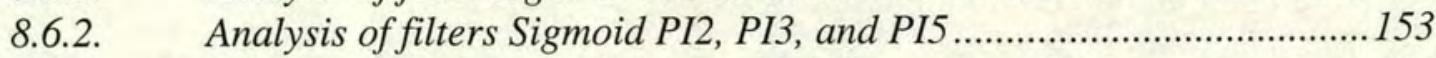

8.6.3. Analysis of main lobe width vs. side lobe suppression ...........................154

8.7. Double edge analysis ............................................................................157

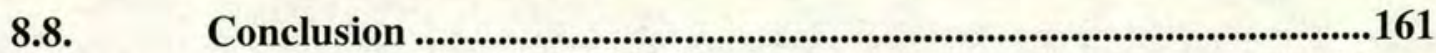

CHAPTER 9. CONCLUSION ................................................ 163

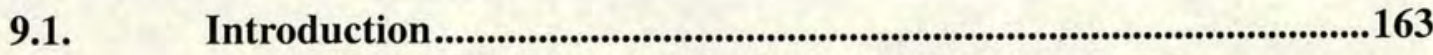

9.2. The main results of this thesis..............................................................163

9.3. Application in a clinical environment ..................................................164

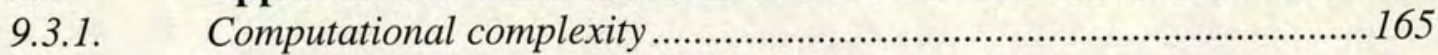

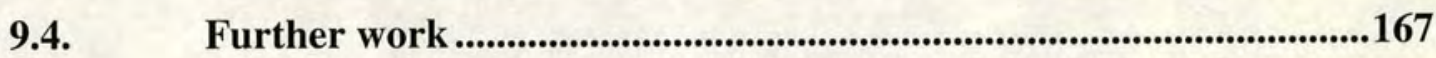

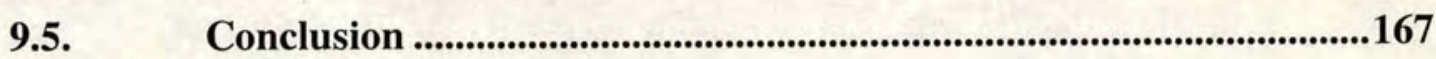

APPENDIX A. BILINEAR INTERPOLATION ............................ 168

A.1. The problem …………............................................................................168

A.2. Definition of the bilinear interpolation ...............................................169

A.3. An alternative approach ........................................................................170

APPENDIX B. ENCLOSED SOFTWARE.................................... 171

APPENDIX C. SOFTWARE FOR IVUS PROCESSING .............. 172

C.1. Main function of program ......................................................................172

C.2. How to make and apply a sigmoid based filter ....................................172

C.2.1. Constructing the filter........................................................................ 173

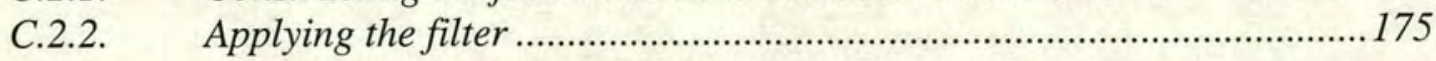

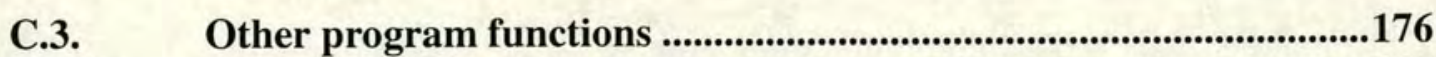

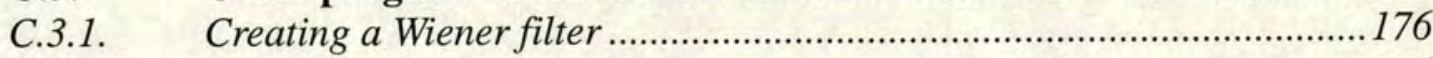

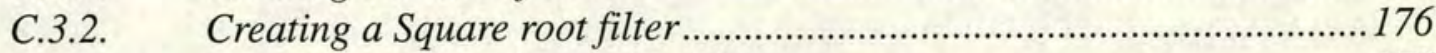

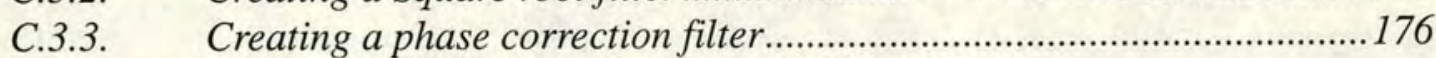


C.3.4. Creating a matched filter

C.3.5. Creating an inverse filter

\section{APPENDIX D. SOFTWARE FOR BITMAP COLOUR} TRANSFORMS

D.1. Operating the program 178

APPENDIX E. SOFTWARE FOR EXTRACTING DATA FROM BITMAPS

E.1. Operating the program 179

E.2. The output format 179

APPENDIX F. IDL SOFTWARE FOR SCAN CONVERSION....... 180

F.1. The different IDL programs .................................................................180

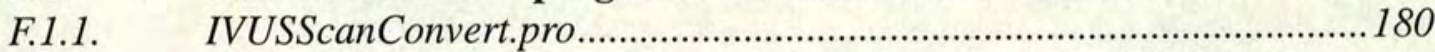

F.1.2. PhantomScanConvert.pro …………………..................................180

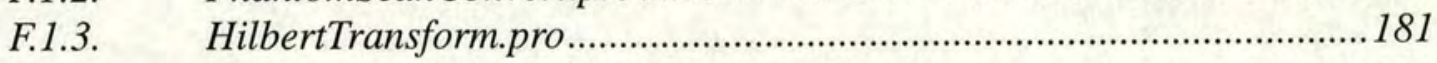

F.2. Characteristics shared by the IDL programs.....................................181

APPENDIX G. OTHER ENCLOSED FILES ............................... 182

G.1. Applications..............................................................................................182

G.2. Ultrasound data samples ......................................................................182

G.2.1. Phantom samples ..........................................................................182

G.2.2. IVUS samples............................................................................183

APPENDIX H. ORIGINAL PUBLICATIONS............................... 184

APPENDIX I. SIGMOID BASED FILTER.................................. 189

REFERENCES ................................................................ 205 


\section{LIST OF FIGURES}

Figure 1.1 Example obstetric ultrasound image ........................................................

Figure 1.2 Example intravascular ultrasound image .................................................... 4

Figure 2.1 Typical medical ultrasound imaging system ............................................ 11

Figure 2.2 Example ultrasound image …................................................................. 12

Figure 2.3 Reflective ultrasound system - transmit (left) and receive (right) ............15

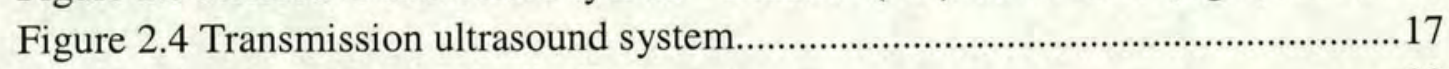

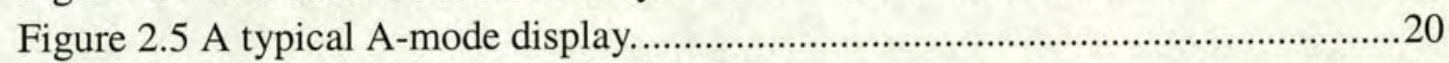

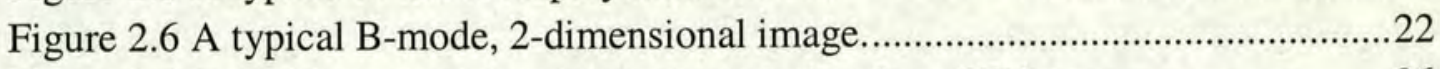

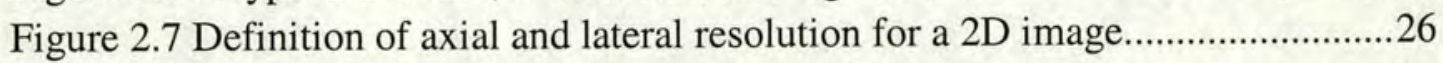

Figure 2.8 Two objects separated by the axial resolution..........................................27

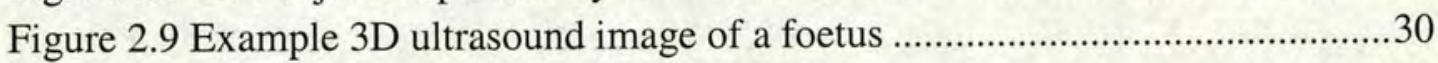

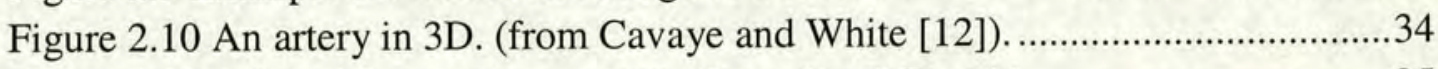

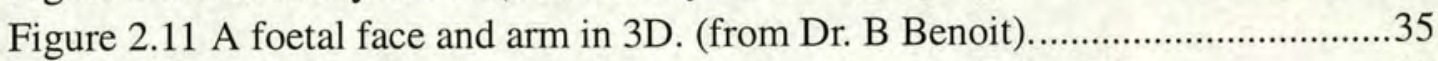

Figure 2.12 A foetal leg in 3D from two angles. (from Dr. B Benoit) ........................ 35

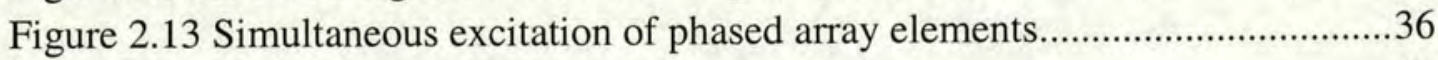

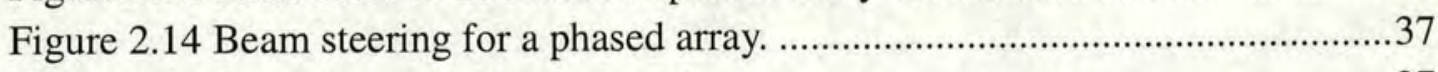

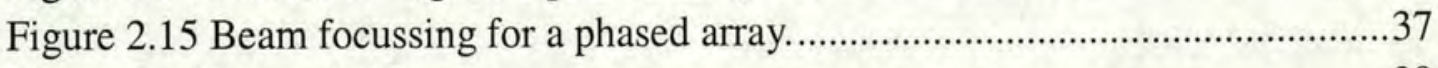

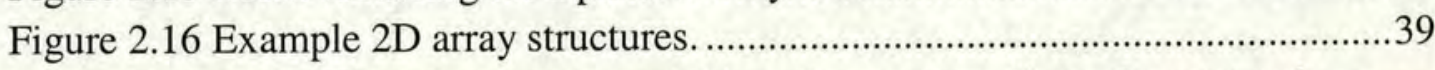

Figure 2.17 An IVUS catheter and arterial imaging system. (from Cavaye and

White [12]).......................................................................................... 40

Figure 2.18 A typical IVUS catheter - slightly forward looking. ..............................41

Figure 2.19 Example IVUS images. (from Cavaye and White [12]). ........................43

Figure 2.20 The structure and some features of arteries. (from Tobis and

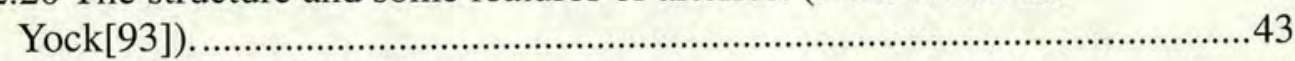

Figure 2.21 Example IVUS image with ringdown and a diverging artery. ................44

Figure 3.1 Typical system to which a filter would be applied...................................47

Figure 3.2 A filter can be used to estimate or reconstruct the original signal. ...........47

Figure 3.3 The assumed model for filter design. ....................................................... 48

Figure 3.4 Matched filter design (right) by time reversal of system impulse response (left). .......................................................................................5

Figure 3.5 Magnitude response of the system impulse response (left), and the matched filter design (right) ......................................................................5 50

Figure 3.6 Example IVUS image (left) and after being filtered with a matched

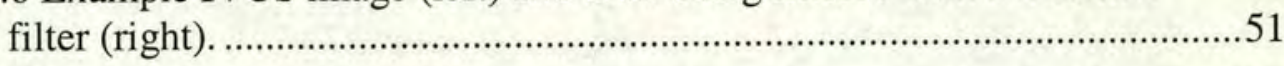


Figure 3.7 An inverse filter in the time domain (right) designed from the system impulse response (left).

Figure 3.8 Magnitude response of an inverse filter (right) designed by inverting the magnitude of the system impulse response (left). .................................52

Figure 3.9 Example IVUS image after being filtered with an inverse filter...............53

Figure 3.10 A Wiener MMSE filter in the time domain (right) assuming SNR = $20 \mathrm{~dB}$ for the system impulse response (left).

Figure 3.11 The magnitude response of a Wiener MMSE filter (right) and the magnitude of the system impulse response (left)

Figure 3.12 Transmission based ultrasound imaging system with an array of receiving elements. .58

Figure 3.13 The model assumed in applying backpropagation. ..................................58

Figure $3.14 \mathrm{~A}$ phase correction filter in the time domain (right) designed from the system impulse response (left).

Figure 3.15 The magnitude response of a phase correction filter (right) designed from the system impulse response (left)....

Figure 3.16 Magnitude response of a phase correction filter response when the filter series is truncated with a rectangular window (left) and a Hann window (right)

Figure 3.17 A square root filter in the time domain (right) designed from the system impulse response (left).

Figure 3.18 The magnitude response of a square root filter (right) designed from the magnitude of the system impulse response (left).

Figure 3.19 Filter Transfer function - designed for the same signal h(n).

Figure 3.20 Filter responses - designed for the same signal h(n)..... .68

Figure 4.1 An ultrasound imaging system (with a perfect reflector).........................71

Figure 4.2 A model of an ultrasound imaging system (as in Figure 4.1) with a perfect reflector. .71

Figure 4.3 A model of an ultrasound imaging system with a target object .72

Figure 4.4 A model of an ultrasound imaging system with a target object with a pseudo inverse filter added to the system .....................................................72

Figure 4.5 A measured system impulse response - for one IVUS system ..................74

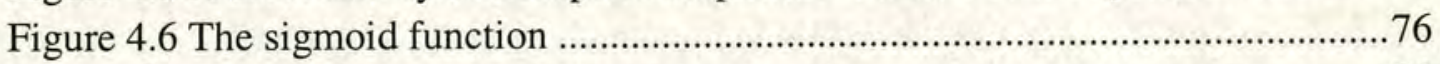

Figure 4.7 Sigmoid based pseudo inversion functions ...............................................76

Figure 4.8 Sigmoid based pseudo inverse functions $(\mathrm{G}=80$ and $\mathrm{T}=0.05)$.................78

Figure 4.9 The lower end of some sigmoid based pseudo inverse functions. $(\mathrm{G}=80$ and $\mathrm{T}=0.05)$ .78

Figure 4.10 The lower end of some modified sigmoid based pseudo inverse

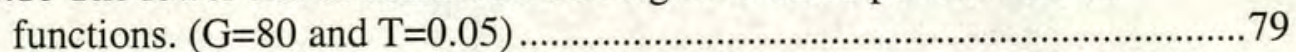

Figure 4.11 Frequency response of an IVUS system frequency response................... 81

Figure 4.12 Filter gain function for two typical sigmoid pseudo-inverse filters .........82 
Figure 4.13 Modified filter gain function for two typical sigmoid pseudoinverse filters

Figure 4.14 Resulting filter impulse responses for two typical sigmoid pseudoinverse filters

Figure 4.15 Resultant power spectra after filtering the system impulse response with two typical sigmoid pseudo-inverse filters

Figure 4.16 Resultant signal after filtering the system impulse response with two typical sigmoid pseudo-inverse filters.

Figure 4.17 An example sigmoid based pseudo inverse filter, truncated with varying length Hann windows

Figure 4.18 Sigmoid filter output functions for a noise free impulse as input, compared with $\mathrm{X}(\mathrm{n}-1) / \mathrm{n}$ filters

Figure 4.19 Sigmoid filter output functions for a noise free impulse as input, compared with Wiener filters

Figure 5.1 In vitro setup for IVUS data collection .....

Figure 5.2 Format of IVUS 2D scan constructed from 1D scanlines .........................93

Figure 5.3 Raw IVUS data in bitmap format..........................................................94

Figure 5.4 IVUS data after being processed for display...........................................95

Figure 5.5 Setup for imaging a perfect reflector (IVUS transducer) .........................96

Figure 5.6 Setup of phantom for data collection ......................................................98

Figure 5.7 Raw phased array data in bitmap format................................................ 100

Figure 5.8 Phantom image after being processed for display (left as displayed by Acuson system), (right as reconstructed by myself)..............................100

Figure 5.9 Reconstructed image of the perfect reflector used ..................................101

Figure 6.1 The perfect reflector as a 2D image of RF ultrasound intensity ..............103

Figure 6.2 The received intensity from a perfect reflector .......................................104

Figure 6.3 The primary pulse received from a perfect reflector................................105

Figure 6.4 The sigmoid based pseudo inverse filter transfer functions presented

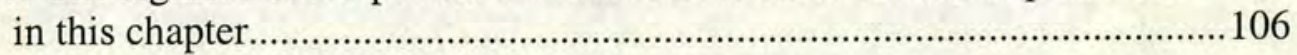

Figure 6.5 Near perfect reflection filtered with modified sigmoid based pseudo inverse filter Sigmoid PI1 ( $\mathrm{G}=60, \mathrm{~T}=0.1, \mathrm{~d}=0.05)$

Figure 6.6 Near perfect reflection filtered with modified sigmoid based pseudo

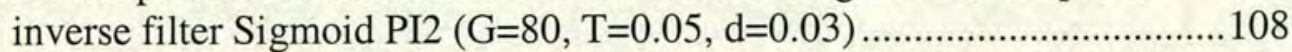

Figure 6.7 Near perfect reflection filtered with modified sigmoid based pseudo

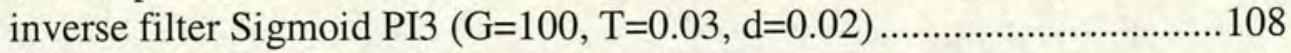

Figure 6.8 Near perfect reflection filtered with modified sigmoid based pseudo inverse filter Sigmoid PI5 ( $\mathrm{G}=100, \mathrm{~T}=0.01, \mathrm{~d}=0.02)$.

Figure 6.9 Near perfect reflection filtered with a matched filter .............................. 111

Figure 6.10 Near perfect reflection filtered with an inverse filter ........................... 111

Figure 6.11 Near perfect reflection filtered with a phase correction filter ...............112

Figure 6.12 Near perfect reflection filtered with a square root based filter..............113 
Figure 6.13 Near perfect reflection filtered with a Wiener filter designed for a noise power level of $100 \%$ of the signal power.

Figure 6.14 Near perfect reflection filtered with a Wiener filter designed for a noise power level of $10 \%$ of the signal power.

Figure 6.15 Near perfect reflection filtered with a Wiener filter designed for a noise power level of $1 \%$ of the signal power.

Figure 6.16 Signal to noise ratios for different filters applied to a near perfect reflection.

Figure 7.1 The angular and distance variation in the perfect reflector image the setup (left) and the reconstructed image (right).

Figure 7.2 Impulse responses of sequential scan lines from a phased array transducer.

Figure 7.3 Power spectra of the impulse response of sequential scan lines from a phased array transducer

Figure 7.4 The example image with a chosen scanline highlighted (the $72^{\text {nd }}$ scanline)

Figure 7.5 The $72^{\text {nd }}$ scanline (after demodulation with a Hilbert transform)

Figure 7.6 The phantom edge in the $72^{\text {nd }}$ scanline (after demodulation with a Hilbert transform)

Figure 7.7 The $72^{\text {nd }}$ scanline filtered with Sigmoid PI1 (after demodulation with a Hilbert transform).

Figure 7.8 The $72^{\text {nd }}$ scanline filtered with Sigmoid PI3 (after demodulation with a Hilbert transform).

Figure 7.9 Right hand side lobe vs. Main lobe width

Figure 7.10 The example image filtered with filters Sigmoid PI1 - left, and Sigmoid PI3 - right (designed with an averaged spectrum and no phase correction).

Figure 7.11 The $72^{\text {nd }}$ scanline filtered with Sigmoid PI1 designed with an averaged spectrum and no phase correction (after demodulation with a Hilbert transform)

Figure 7.12 The $72^{\text {nd }}$ scanline filtered with Sigmoid PI3 designed with an averaged spectrum and no phase correction (after demodulation with a Hilbert transform)

Figure 7.13 Right hand side lobe vs. Main lobe width for filters designed with an averaged spectrum and no phase correction

Figure 8.1 The example image with no filtering applied

Figure 8.2 Example image with lines of interest highlighted

Figure 8.3 Example image after being filtered with filter Sigmoid PI1 140

Figure 8.4 Example image after being filtered with filter Sigmoid PI2 141

Figure 8.5 Example image after being filtered with filter Sigmoid PI3 142

Figure 8.6 Example image after being filtered with filter Sigmoid PI5

Figure 8.7 Example image after being filtered with a matched filter 144

Figure 8.8 Example image after being filtered with a phase correction filter 145 
Figure 8.9 Example image after being filtered with a square root based filter.........146

Figure 8.10 Example image after being filtered with an inverse filter ......................147

Figure 8.11 Example image after being filtered with a Wiener filter designed for $100 \%$ noise power

Figure 8.12 Example image after being filtered with a Wiener filter designed for $10 \%$ noise power.

Figure 8.13 Example image after being filtered with a Wiener filter designed for $1 \%$ noise power.

Figure 8.14 Intensity of reconstructed image in region 1 - with no filtering applied

Figure 8.15 Intensity of reconstructed image in region 1 - filtered with filter Sigmoid PI1

Figure 8.16 Intensity of reconstructed image in region 1 - filtered with filter Sigmoid PI2

Figure 8.17 Intensity of reconstructed image in region 1 - filtered with filter Sigmoid PI3

Figure 8.18 Intensity of reconstructed image in region 1 - filtered with filter Sigmoid PI5

Figure 8.19 Left hand side lobe vs. Main lobe width .156

Figure 8.20 Right hand side lobe vs. Main lobe width .157

Figure 8.21 Intensity of reconstructed image in region 4 - with no filtering applied .158

Figure 8.22 Intensity of reconstructed image in region 4 - filtered with filter Sigmoid PI1

Figure 8.23 Intensity of reconstructed image in region $4-$ filtered with filter Sigmoid PI2

Figure 8.24 Intensity of reconstructed image in region 4 - filtered with filter Sigmoid PI3

Figure 8.25 Intensity of reconstructed image in region 4 - filtered with filter Sigmoid PI5

Figure A.1 Possible arrangements of 1D scanlines on a polar grid

Figure A.2 Bilinear interpolation problem on a polar grid 


\section{LIST OF TABLES}

Table 3.1 Exponents of Fourier components for various filters. .................................65

Table 4.1 Names assigned to parameter sets for sigmoid based PI filters ....................80

Table 7.1 Main lobe width vs. side lobe suppression ............................................128

Table 7.2 Main lobe width vs. side lobe suppression for filters with an averaged spectrum and no phase correction ............................................................134

Table 8.1 Main lobe width vs. side lobe suppression ................................................156 


\section{ABBREVIATIONS}

AWGN Additive, white, Gaussian noise.

CAT Computerised axial tomography.

FIR Finite impulse response.

FPS Frames per second.

IIR Infinite impulse response.

MMSE Minimum mean square error.

MRI Magnetic resonance imaging.

NMR Nuclear magnetic resonance.

PCG Phonocardiogram.

PET Positron emission tomography.

P.I. filter Pseudo inverse filter.

PSP Paint Shop Pro, a shareware graphics manipulation program.

RMS Root mean square.

SNR Signal to noise ratio.

SVD Singular value decomposition.

TGC Time gain compensation.

VCL Visual component library, user interface code supplied with Delphi. 


\section{LIST OF PRINCIPAL SYMBOLS}

$\delta(n) \quad$ A dirac delta function. A perfect impulse of infinitely short duration.

$\mathrm{F}(\omega) \quad$ The value of a Fourier component of a signal, at angular frequency $\omega$.

$\mathrm{h}(\mathrm{n}) \quad$ The impulse response of a distorting channel.

$H(\omega) \quad$ The frequency response of a distorting channel.

$\mathrm{x}(\mathrm{n}) \quad$ The taps of a filter.

$\mathrm{X}(\omega) \quad$ The frequency response of a filter.

$y(n) \quad$ The output of a filter $x(n)$, given $h(n)$ as the input to the filter.

$\mathrm{Y}(\omega) \quad$ The frequency response of a distorting channel plus a filter. 


\section{Medical TERMINOLOGY}

Balloon angioplasty The process of inserting a balloon in an artery in order to increase the width of the artery.

Atherectomy A procedure to remove plaque from the inside of an artery, using a special cutting device.

Thrombus A blood clot inside an artery, which can restrict the area inside the artery that is available for blood flow.

Intima The inner most layer on a healthy artery.

Media An arterial layer, between the intima and the adventitia.

Adventitia An arterial layer, outside of the intima and media. This is made of fibrous tissue.

Atherosclerosis A disease affecting arteries, causing the build up of deposits on the intimal surface.

Plaque The collective name given to atherosclerotic build ups on the inner intimal surface of the artery.

Electrocardiogram The monitoring of electrical signals from the heart. Used principally for monitoring the rate of heartbeats. 


\section{Chapter 1.}

\section{INTRODUCTION}

\subsection{Introduction}

Ultrasound imaging is widely used in many areas in medicine [1]. It provides a safe [2] and efficient means for medical personnel to obtain information about a patient. Unfortunately the images produced by ultrasound are often of a poor quality when compared to other imaging systems such as X-rays, magnetic resonance imaging (MRI), (or nuclear magnetic resonance (NMR) imaging), positron emission tomography (PET), and computerised axial tomography (CAT) [3, 4].

This work details the novel development and application of a filter structure to a new area - medical ultrasound images, in an attempt to improve the quality of the ultrasound images. This filter is a pseudo inverse filter, based on a sigmoid function which was developed for use with foetal phonocardiograms (PCG). Results are presented from modifying this filter and applying it to medical ultrasound images. Comparisons with the results of other filters previously proposed for application to medical ultrasound images are also presented. This work constitutes a contribution to knowledge, in that a filter design technique has been modified and adapted in a new way. The modified filter has been applied to a new area, and extensive comparisons with other filtering techniques have been performed. These comparisons are subjective and objective - using measures such as the apparent widths of surfaces, the (axial) separation of adjacent surfaces, and image contrast. These objective measures give quantifiable measures of the performance of the various filters, and provide a solid basis for comparisons. These comparisons show that the proposed filter performs well with medical ultrasound images. 


\subsection{Medical ultrasound}

Medical ultrasound is used to image many different parts of the human body $[1,5]$, such as the foetus $[5,6,7,8]$, the heart $[5,9]$, liver and other small parts $[1,5,10$, $11]$, and arteries $[5,12,13]$ such as the carotid artery $[14,15]$. It is attractive as a diagnostic tool because it is cheaper than other imaging techniques [16] and is also considered to be safe [2]. An example of a medical ultrasound image is shown in Figure 1.1. This is an obstetric image of a foetus.

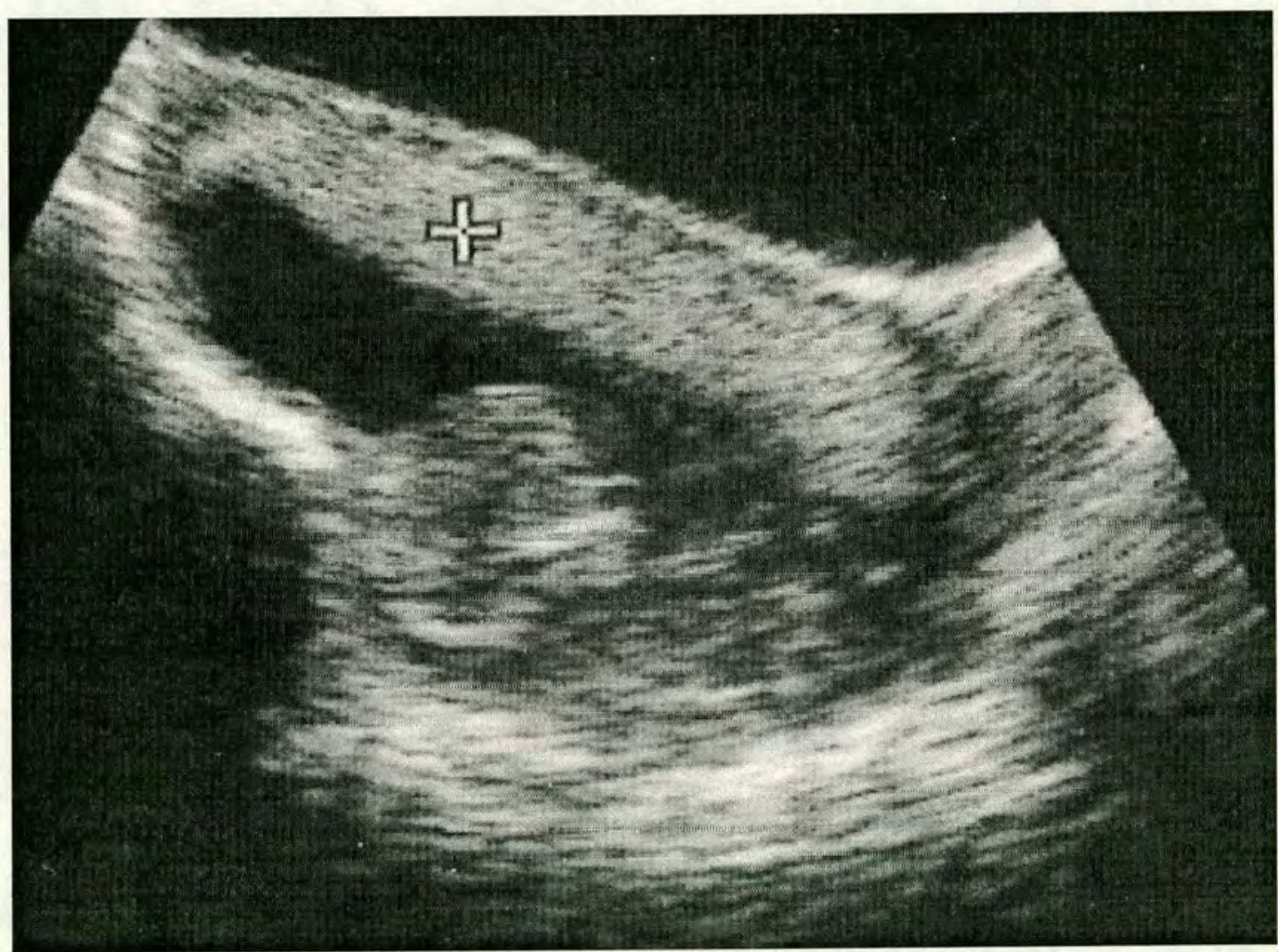

Figure 1.1 Example obstetric ultrasound image

Medical ultrasound can provide medical personnel with information about the texture of the target objects $[17,18,19]$ (e.g. arterial walls [20, 21, 22]), whether certain objects are present or not (e.g. kidney stones [1], calcified plaque on arterial walls [23]), and the size of the objects. Example areas where measurements from ultrasound are used include foetal dimensions [1, 6] (e.g. head width, head 
circumference, and crown-rump length), and measurements of the width (or occlusion) of the arteries [24, 25, 26, 27] such as the carotid artery [15, 28]. The accuracy of these measurements is important for clinical decision making.

The accuracy of measurements from medical ultrasound images is limited by the resolution of the image. The resolution of the image may be expressed in each of 3 dimensions, axial (also called 'range'), lateral, and the second lateral dimension which in $2 \mathrm{D}$ images is measured only by analysing multiple images. Of these dimensions, the best resolution is obtained in the axial dimension [29], since that is not affected by beam spreading; it is limited primarily by the duration of the transmitted pulse. Therefore in order to improve the accuracy of any measurements, a clinician can make the beam perpendicular to the objects or surfaces being measured [29].

However it is possible to improve the axial resolution below the limit imposed by the duration of the transmitted pulse. This is possible by appropriate filtering of the ultrasound pulse to shorten the effective pulse duration. This filtering is usually done after the signal is received and prior to display, but it can potentially be done prior to transmission. This thesis is concerned with the design of appropriate filters to reduce the duration of the transmitted ultrasound pulse (by filtering after the signal is received), and thereby improving the (axial) resolution of the resulting image.

\subsubsection{Intravascular ultrasound}

Intravascular ultrasound (IVUS) is one example of the many medical uses of ultrasound [12, 13, 30, 31, 32]. IVUS is an invasive technique, where the ultrasound catheter is inserted into the patient's artery, and images are obtained from within the artery (looking outwards). This technique has become widely accepted [16], particularly for the monitoring of invasive surgical techniques e.g. balloon angioplasty and atherectomy. An example IVUS image is shown in Figure 1.2. 


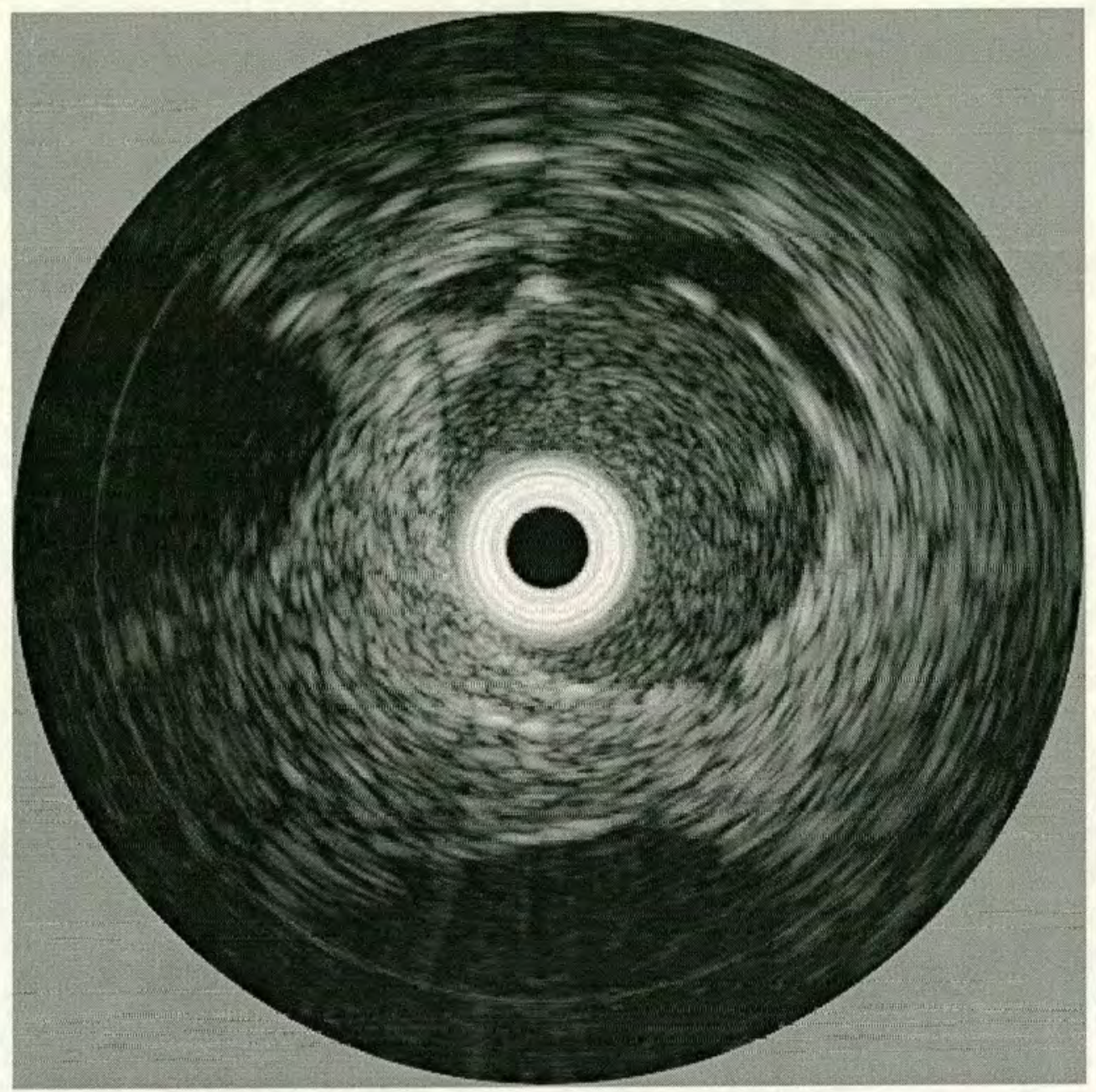

Figure 1.2 Example intravascular ultrasound image

IVUS is usually performed at high frequencies (e.g. $30 \mathrm{MHz}$ ), in order to increase the resolution of the ultrasound image. Most medical ultrasound images use frequencies between $1 \mathrm{MHz}$ and $10 \mathrm{MHz}$. The higher frequency also causes greater attenuation which gives a reduced depth of vision in the image - making it unsuitable for many ultrasound applications. In IVUS the ultrasound does not need to penetrate far, and the extra resolution of the image is important [33] since the target object (the artery) is small and the details of the artery are even smaller. 


\subsection{Ultrasound processing}

In order to improve the quality of medical ultrasound images, various filtering techniques have been employed to alter the appearance of the image $[34,35,36]$. There are also ongoing attempts at constructing real time three dimensional images $[37,38,39]$ which provides a greater degree of visualisation in some applications.

\subsubsection{Filtering of ultrasound}

Many attempts have been made at filtering ultrasound. Some techniques attempt to smooth the speckle in the images $[40,41,42]$. This technique has been refined so that it attempts to preserve the edges while smoothing the speckle between the edges $[43,44,45,46,47]$. Some of these algorithms rely on the detection or estimation of edge locations. However medical personnel are reluctant to have the speckle removed from the images, as the speckle often gives an indication of the texture of the imaged objects - which can potentially provide valuable clinical information. This has been the topic of some research $[17,18,19,20,21,22,48,49,50,51,52$, 53].

Other attempts at filtering ultrasound have concentrated on the resolution of the image. This has been applied successfully in the three following areas:

- to non medical applications of ultrasound, such as non destructive testing and fault detection [54];

- to images of wires using medical scanning [55];

- to transmission based ultrasound (e.g. Zhu and Steinberg [56]).

However each of these three situations is a significantly easier problem than reflective ultrasound in a medical environment, since each of these situations gives only one pulse at a time - which is well isolated from other pulses. In medical applications of reflective ultrasound the pulses are continually reflected and scattered 
from every boundary in the target region (such as cellular walls), this causes a significant amount of interference which appears in the image as speckle.

Some other researchers (e.g. Shen and Ebbini $[57,58,59,60,61]$ ) have attempted forms of pseudo inverse filtering on reflective ultrasound after deliberately spreading the ultrasound pulse (using spreading codes, in a manner similar to a spread spectrum system). This resulted in a pulse duration, after filtering, that was approximately equal to its original duration. The motivation for spreading the pulse was to allow simultaneous acquisition of multiple scanlines for faster 3D imaging - with each scanline encoded with a given spreading code. In this application the filters were required to isolate the given scanlines rather than to reduce the pulse duration.

\subsubsection{Three dimensional imaging of ultrasound}

Three dimensional images constructed from ultrasound images can be useful in many medical situations, and can provide additional information that is not readily (and accurately) available from conventional $2 \mathrm{D}$ images [27, 62]. The estimation of foetal volume (indicating foetal growth) is one such example. The largest benefits of $3 \mathrm{D}$ images for medical personnel are likely be in the ease of visualisation, and the reduced training time due to the images being more intuitive to understand than $2 \mathrm{D}$ images. In particular, the spatial relationship between objects can be observed with greater accuracy and ease from a 3D image, than from a collection of 2D images.

Two large problems with generating 3D images from medical ultrasound are the need for the acquisition of large amounts of ultrasound data (which is limited in part by the speed of sound), and the difficulty of extracting the edge or surface information from the ultrasound $[23,25,26,63,64,65]$. The second problem arises due to the poor signal qualities of ultrasound when compared to other forms of medical imaging (such as MRI). These poor qualities include:

- poor signal to noise ratio; 
- poor dynamic range;

- variations in attenuation with propagation through different materials;

- variations in speed with propagation through different materials.

These problems present a significant challenge to the construction of 3D images. False edges are often detected in the ultrasound data, which are caused by the high noise levels [66]. The accurate and reliable automatic detection of surfaces and edges from within medical ultrasound images remains a challenge $[23,25,26,65,67,68$, $69,70,71]$.

Despite the problems with medical ultrasound 3D images, systems have been developed that will provide 3D images - but not in real time $[53,59,60,61,72,73$, $74,75,76,77,78,79]$. Also, the systems often require some degree of manual control, such as: setting the thresholds for registering the presence of an edge (or not); finding the first edge in the image; and sometimes the manual removal of erroneous edges, and edges outside the region of interest [75].

\subsection{Organisation of the thesis}

Chapter 2 of this thesis details the types of systems used with medical ultrasound imaging systems, and in particular with intravascular ultrasound (IVUS). It also covers various ultrasound image display modes. Chapter 5 describes the particular systems that were used to collect the ultrasound data that is presented in this thesis.

Chapter 3 describes a variety of linear filtering techniques that have been applied to medical ultrasound imaging systems by various researchers, and the theory behind these filters. Some of their limitations in this application are also described. Chapter 4 describes an alternative filter design technique that is proposed for application to medical ultrasound imaging systems. This filter is a sigmoid based pseudo inverse filter, which has never before been applied to ultrasound. 
Chapter 6, 7, and 8 report results from applying the proposed filter to various ultrasound signals. These are: near perfect ultrasound reflections (Chapter 6); an ultrasound image of a phantom (Chapter 7); and intravascular ultrasound images from a rabbit (Chapter 8). This provides a variety of tests for the filter, with varying levels of prior knowledge about the image target (allowing good comparisons between the final image and the known target object), and varying levels of similarity to a clinical imaging environment (where the image target details are largely unknown). It is important to test the filter with clinical data (or a good approximation such as the rabbit IVUS data), but this has the difficulty that the exact pathology of the target in the image is not known. The phantom and the near perfect reflector images therefore serve the purpose of allowing the filter to be tested in an environment where it is known for certain what the image should contain, and therefore comparisons between the filtered image and the "ideal" image can be made with greater confidence.

\subsubsection{The appendices}

Appendix A describes the bilinear interpolation algorithm, which is used in the image reconstruction process.

Appendix B provides an overview of the software that implements the algorithms presented in this thesis. This software is included (with source code) on an accompanying compact disc (CD), and includes all software necessary for duplicating the results presented in this thesis.

Appendix $\mathrm{C}$ provides instructions on using the principal program utilised in this thesis. This program is able to construct, apply, and analyse each of the filters described in this thesis.

Appendix D and Appendix E describe additional programs used in this thesis. They perform only data format conversions, and are provided and described only for completeness. 
Appendix F describes a program that implements the image construction algorithm, known as "scan conversion".

Appendix $\mathrm{G}$ describes remaining files on the $\mathrm{CD}$ (those not already described in previous appendices). This includes samples of ultrasound data from near perfect reflectors, a phantom, and some IVUS data.

Appendix $\mathrm{H}$ reproduces previously published papers by the author, related to the work presented in this thesis. Appendix I reproduces a section from a Ph.D. previously published by $\mathrm{H}$. Basil [80] that describes the original presentation of the sigmoid based pseudo inverse filter. 


\section{Chapter 2. ULTRASOUND IMAGING}

\subsection{Introduction}

Ultrasound imaging is widely used in medicine $[1,5]$. Other imaging systems are also used such as X-rays, MRI (or NMR), PET, and CAT [3]. Despite the availability of alternative imaging systems, ultrasound continues to enjoy wide acceptance and usage [81]. The principal attractions of ultrasonic imaging are:

- it is considered by medical experts to represent no hazard to the health of a patient - unlike other systems [82];

- it is cheaper than other imaging systems [16];

- it is good for imaging soft tissue structures in detail. In this regard ultrasound outperforms X-rays;

- portable ultrasound imaging systems are available [29];

- measurements of movement can be made - e.g. rates of blood flow.

Ultrasound imaging is considered to be safe. Although some reports have been presented to indicate that ultrasound is hazardous, these reports have proven to be unfounded [2]. The current prevailing medical opinion is that exposing a patient to typical clinical levels of ultrasound represents no danger to a patient's health. This provides the opportunity for repeated clinical examination with ultrasound - whereas other systems with a higher level of danger cannot safely examine patients multiple times. 


\subsection{General ultrasound imaging}

Ultrasound can be used to construct images of human anatomy due to the interaction between the body tissue and the ultrasound vibrations [83, 84, 85]. It is used for imaging many parts of the human body $[1,5,65]$ including the heart $[9,86]$, foetus $[1,7]$, vascular systems $[3,12,14]$, and other "small parts" (e.g. kidneys, liver) [1, 5, 10].

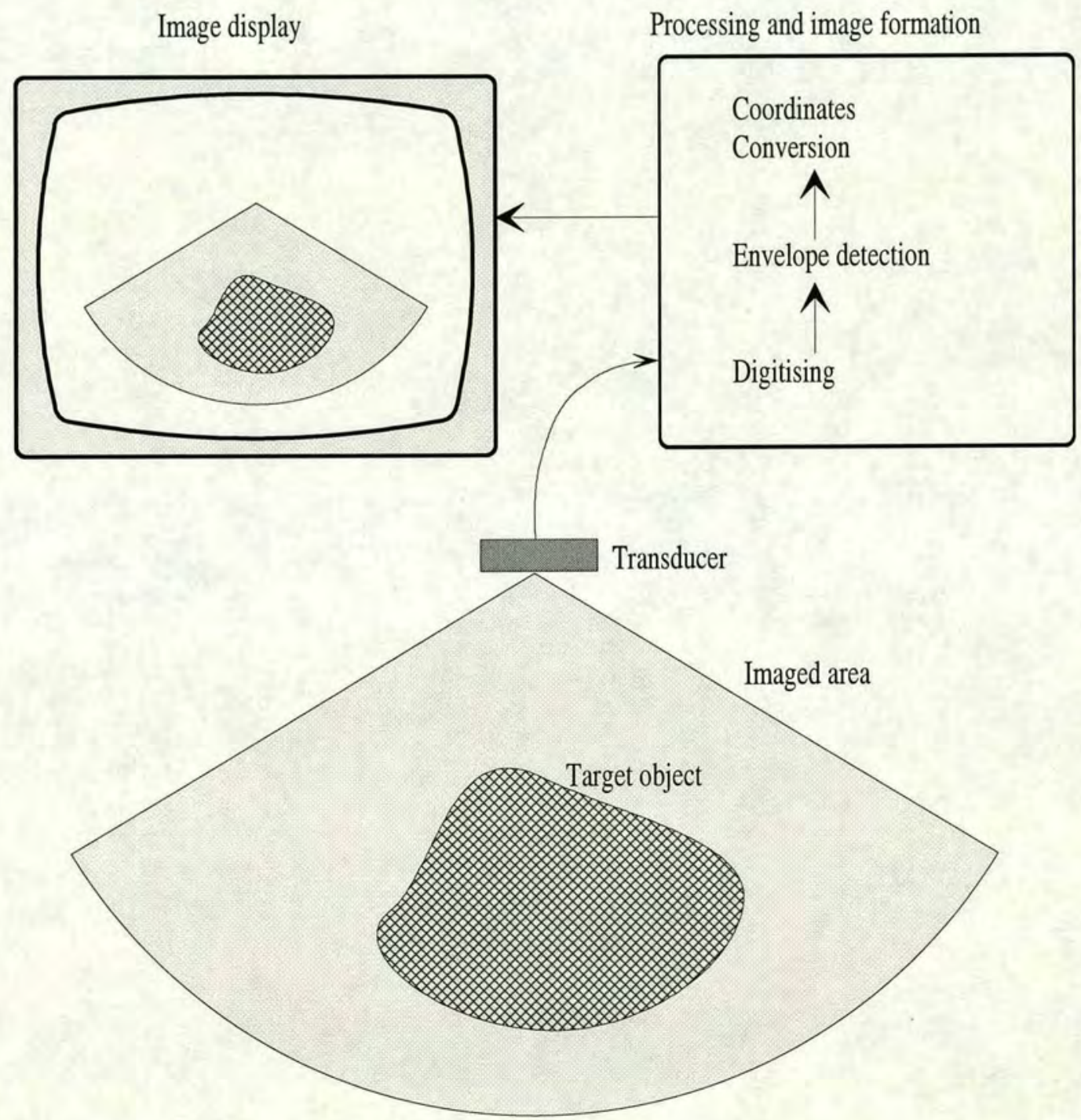

Figure 2.1 Typical medical ultrasound imaging system

A typical ultrasonic imaging system as used in medical applications is shown in Figure 2.1. Normally the target object would be inside a human patient. 
Ultrasound vibrations are most commonly created by applying a short duration voltage pulse to a piezoelectric crystal, which then vibrates at an ultrasonic frequency (in medical applications this is typically $1 \mathrm{MHz}$ to $30 \mathrm{MHz}$ ). The vibrations are typically damped such that they will continue for a few cycles (or wavelengths) only.

Piezoelectric crystals can also be used to detect ultrasound vibrations through the reverse mechanism, of the ultrasonic vibration creating a voltage.

The ultrasound is used to scan the object, and then the received signals are used to construct and display an image for the user. The user must interpret the image to obtain clinical information. The extraction of features and edge locations, and the interpretation of textural information is a non-trivial task as illustrated by the example ultrasound image in Figure 2.2.

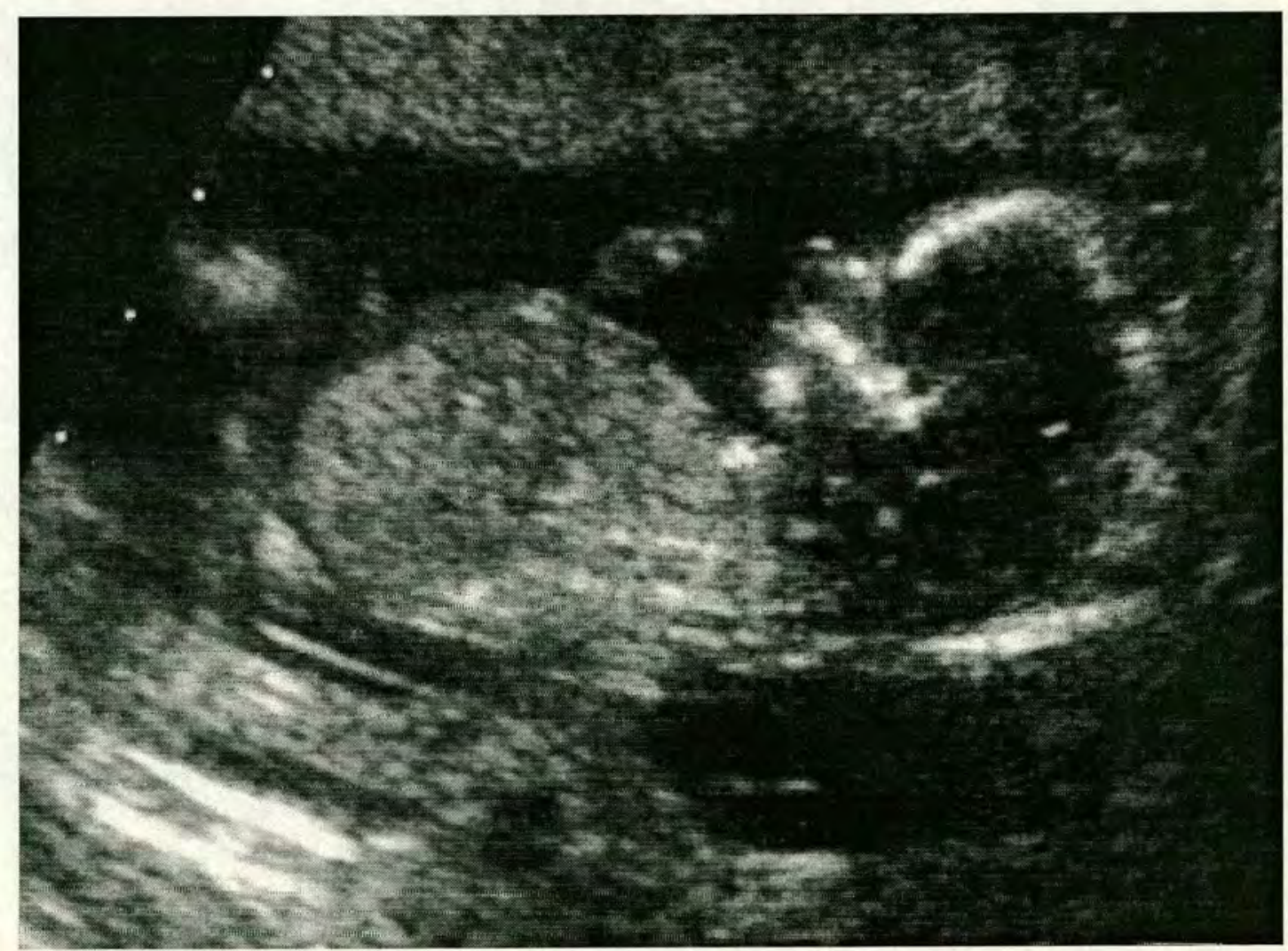

Figure 2.2 Example ultrasound image 


\subsubsection{Required system components}

Several components are required in order to have a functioning ultrasonic imaging system - such as in Figure 2.1. The components are:

- a source or transmitter of ultrasonic vibration. Typically this is one or more piezoelectric crystals with applied voltage pulses of short duration. In Figure 2.1 the transducer is the transmitter;

- a detector or receiver of ultrasonic vibration. Typically this is one or more piezoelectric crystals. The received voltages are typically amplified, and digitised. If there is more than one receiving element then the signal from the various receiving elements may be combined (usually by delaying and summing the signals). In Figure 2.1 the transducer is the receiver - as well as the transmitter;

- a good acoustic coupling between the transmitter and the medium, and also between the receiver and the medium.

- a processing unit to convert the received ultrasound vibrations into an image suitable for display and human interpretation. On different systems this section can vary greatly in its complexity;

- a visual display unit on which to display the images.

In some systems (e.g. Figure 2.1), the receiver and the transmitter may be the same crystal. In this case, the transducer will alternately work as first the transmitter, and then the receiver.

\subsubsection{Differences in medical ultrasound imaging systems}

There are many variations in the characteristics of medical ultrasonic imaging systems. Often the differences are designed to make a particular system well suited to 
one particular medical application. Some of the important distinguishing characteristics in medical imaging systems are:

- whether the system uses ultrasound transmission or reflection;

- the imaging display mode (e.g. A-mode, B-mode, M-mode, 3D);

- the type of transducer (particularly whether it has only a single receiving element, or an array of elements).

Other factors also distinguish different medical systems such as the frequency used, and whether the system is invasive or not. These differences are less important from an engineering and signal processing standpoint. The following sections look at these differences, and their importance and relevance in ultrasonic imaging.

\subsection{Reflection and transmission based ultrasound}

\subsubsection{Reflective ultrasound}

Reflective ultrasound imaging is based on the pulse echo principle. In order for the ultrasound to be received by the receiving transducer element(s) the ultrasound must be reflected (or scattered) by some object(s). Therefore any object that does not reflect ultrasound will not appear in a reflective ultrasound image (that is, it will appear black or invisible). The amount of incident ultrasound that is reflected from any boundary where there is a change in acoustic density is given [87] by equation 2.1:

$\left|A_{\text {reflected }}\right|=\left|\frac{c_{2}-c_{1}}{c_{1}+c_{2}}\right| \cdot\left|A_{\text {incident }}\right|$

where $c_{2}$ is the speed of sound in the material that the ultrasound pulse is trying to enter, and $c_{1}$ is the speed of sound in the material that the ultrasound pulse is 
currently (or previously) in. The symbols $A_{\text {incident }}$ and $A_{\text {reflected }}$ represent the amplitudes of the incident and reflected ultrasound waves respectively.

Erroneous multiple reflections can occur and be detected [29]. Distinguishing multiple reflections from (normal) single reflections can be difficult or impossible, so all reflective ultrasound imaging systems assume that all received signals are reflected once only. With multiple reflections this assumption is false, which causes non-existent surfaces to be displayed. These reflections can be called "ghost" surfaces.

The principal advantage of reflective ultrasound systems (over transmission based systems) is that a single piezoelectric crystal element (or an array of crystal elements) can be used as both the transmitter and the receiver. This allows a system to be somewhat easier to use clinically, in particular the transducer can be handheld and have greater freedom of movement. It also has the significant clinical advantage that the ultrasound signals do not need to penetrate fully through the body. This means that if a signal is severely attenuated at some point in the target, then all points between the transducer and the attenuation point can still be imaged.
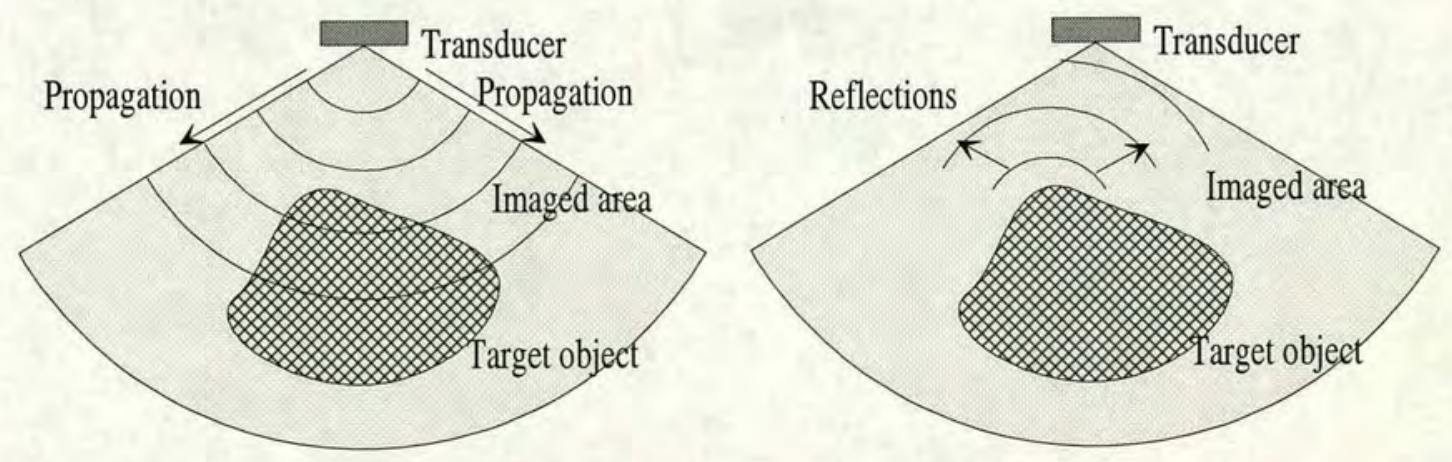

Figure 2.3 Reflective ultrasound system - transmit (left) and receive (right)

The transducer must alternately transmit and then receive. It switches between the two operations. After transmitting a pulse, it operates in receive mode until the signals from the furthest imaged distance have returned. The time $t$, in seconds, for a 
signal to return to the transducer from an object can be calculated using the simple equation given in equation 2.2 :

$t=2 d / c$

The factor 2 in equation 2.2 is due to the fact that for an object at a distance $d$, in metres from the transducer, the ultrasound must travel to the object, and back to the transducer - a return journey. The speed of sound in the body $c$, in $\mathrm{m} / \mathrm{s}$, will vary somewhat with the density of the tissue it passes through. However the value of 1540 $\mathrm{m} / \mathrm{s}$ is widely used as an average speed [29, 88], and this serves as an adequate approximation. Therefore, if we use the average speed of $c=1540 \mathrm{~m} / \mathrm{s}$, and the largest distance $d$ that we wish to image, then we can calculate how long the transducer must operate as a receiver, before it can again operate as a transmitter.

The transducer will receive returning ultrasound signals for the duration of the time $t$ in equation 2.2. Whether the received signal is larger (or smaller) will be determined by the presence (or absence) of strong reflecting elements within the imaged area.

It is possible for some residual ultrasound to reach the transducer after a longer time than is anticipated - causing an imaging error by adding to the signal of a subsequent transmit-receive cycle. Such components could come from multiply reflected ultrasound, or from very distant reflecting objects. These erroneous reflections will usually be weak compared to the primary reflections, and will usually be classified as noise although they may appear as "ghost" surfaces.

\subsubsection{Transmission ultrasound}

Transmission based ultrasound imaging is based on detecting the transmission of ultrasound, and not reflection. The component of ultrasound that is received by the receiving transducer element(s) is the transmitted component, that is, the component of the ultrasound that passes through the target object. Therefore any object that does 
not transmit ultrasound will not appear in a transmission ultrasound image (that is, it will appear black or invisible). A simplified transmission system is shown in Figure 2.4 .

In transmission based ultrasound, there is always a separate receiver and transmitter. The imaged object must be able to be placed between the transmitter and the receiver.

Also, the imaged object (and any surrounding body of material) must be small enough that the ultrasound can pass through the body and the object without being severely attenuated. Some objects may fail to transmit ultrasound due to their structure. One example of this is bone, another is an interface between human tissue and gas or air.

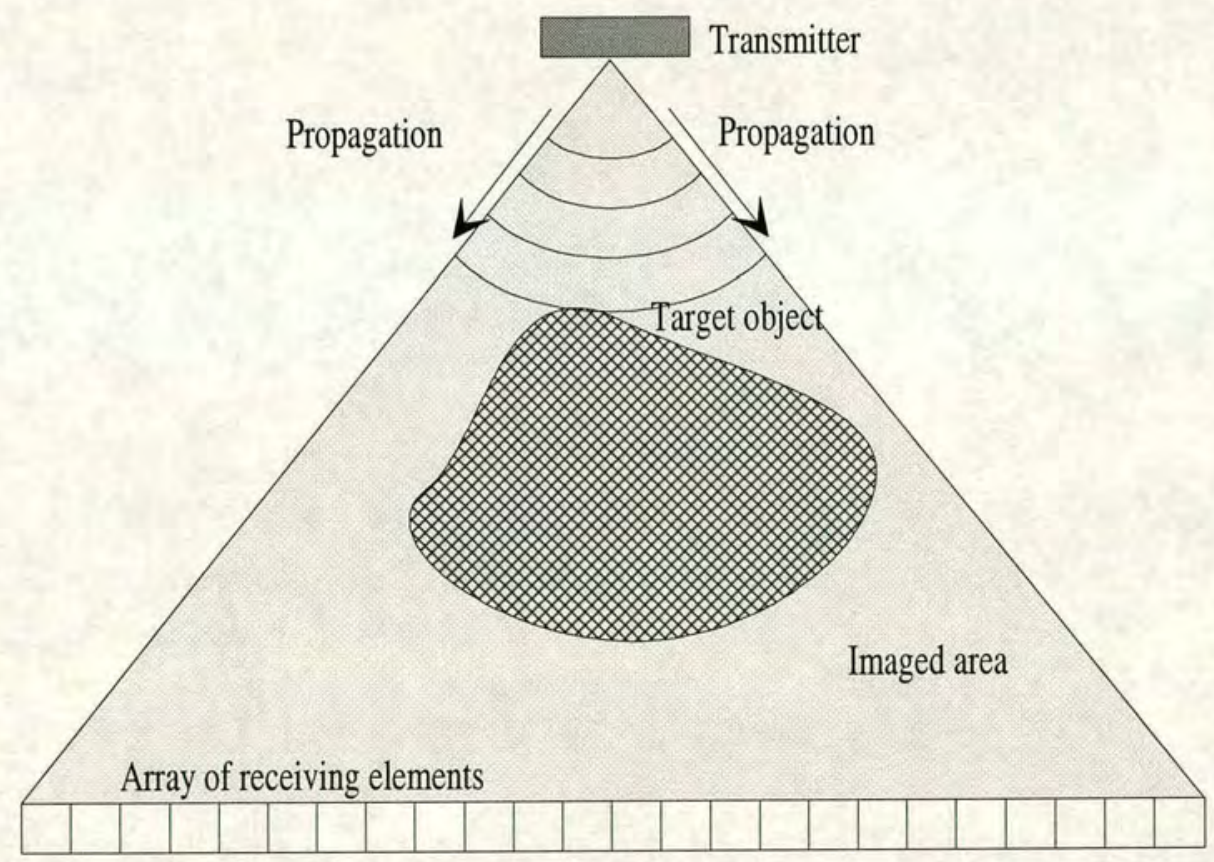

Figure 2.4 Transmission ultrasound system.

Figure 2.4 shows an array of receiving elements. This is the usual case, although it is possible to construct an image using only a single receiving element, and by repeatedly moving the element to receive ultrasound pulses at different locations. This could be thought of as simulating an array - it will work well only if the target 
is stationary. It is also possible to combine these two methods, and have a moving array of elements e.g. an array constructed in 1 dimension (left to right), with the whole array moving in a $2^{\text {nd }}$ dimension (up and down).

The time taken for an ultrasound pulse to pass through the system (from the transmitter to the receiver) in a transmission based ultrasound system is given by equation 2.3:

$t=d / c$

Where $d$ is the distance between the transmitter and the receiver, and $c$ is the speed of sound in the medium. It is worth noting that the duration for which the receiver will receive any significant signal is determined not by the pulse travel time, but by the duration of the pulse transmitted (generally much less than the pulse travel time). This enables a transmission based ultrasound system to (potentially) have a greater frame rate than a reflective ultrasound system.

The array of receiving elements can be a planar array, or a curved array. The geometry must be accounted for in the processing of the signals, but the basic techniques and image qualities remain the same.

\section{Problems with transmission based ultrasonic imaging}

Medical applications of transmission based ultrasound systems are uncommon [29]. This is due to the difficulties involved with this imaging system. These difficulties include:

- the separate transmitter and receiver make the device less easy to manoeuvre in a "handheld" manner;

- misalignment between the transmitter and receiver can (in the worst case) cause no signal to be received. This makes manoeuvring the transmitter and receiver 
more awkward for clinicians than is the case for many reflective ultrasound systems;

- ultrasound is almost completely reflected by any tissue-gas interfaces in the image target or body. Ultrasound is also severely attenuated by bone. This limits the number of possible medical applications;

- in some applications, the ultrasound has to travel further than in a reflective ultrasound system. This adds extra signal attenuation which in turn restricts the usable ultrasound frequencies (and thereby restricts the image resolution);

- refraction and diffraction of the ultrasound beam may cause greater image inaccuracies than in the reflective ultrasound case.

\subsection{Imaging formats}

There are a variety of image display formats for ultrasound. These formats have evolved over time, and have (to some extent) followed advances in technology particularly the advent of greyscale and colour computer monitors.

Each different imaging modality has its own merits, and areas of application. Each of the following modalities are all currently used in clinical applications.

\subsubsection{A-mode imaging (1D)}

A-mode (or amplitude-mode) imaging is the simplest form of ultrasonic imaging. It is inherently one dimensional, and therefore has only limited applicability. However an understanding of A-mode imaging helps a user to understand all other display modalities.

A-mode image applications include examinations of the eye, and the midline of the brain. They are sometimes available as a secondary display mode on machines with other imaging modalities. 
A-mode images appear on a screen in a similar manner to a voltage signal on an oscilloscope. There is a single trace across the screen at any given time as seen in Figure 2.5. The $x$-axis of the display is time, and the $y$-axis is the received intensity of the ultrasound signal after demodulation.

The displayed trace of an A-mode image represents the ultrasound signal returned from a 1-dimensional line of view. Any reflective objects encountered along this line of view will provide a reflection, which then appears on the display as peaks or elevated regions.

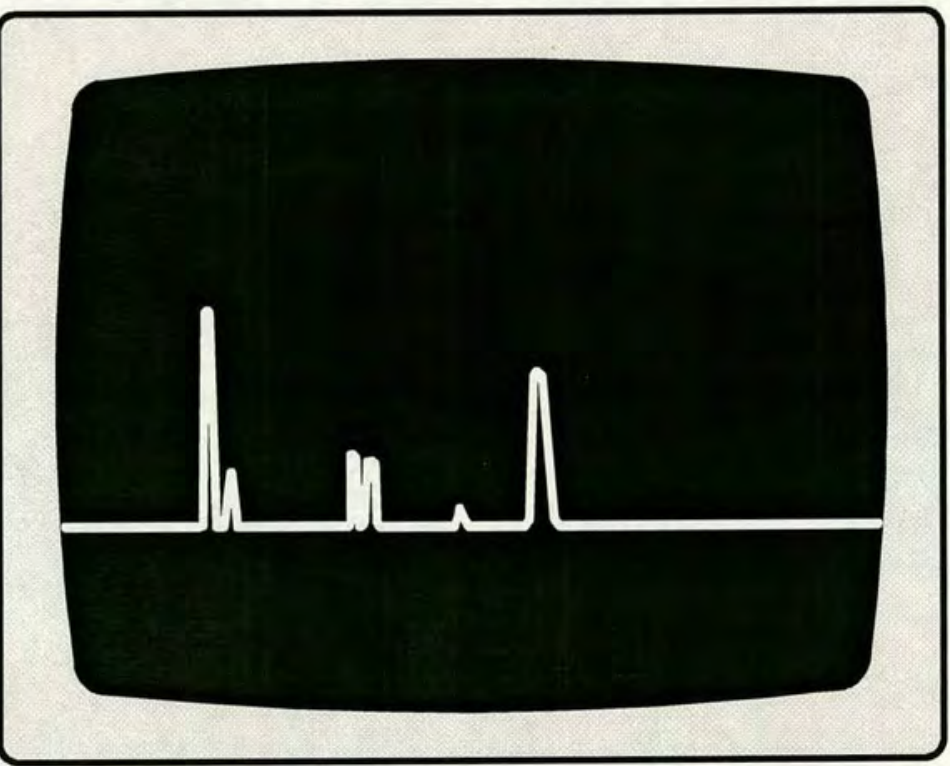

Figure 2.5 A typical A-mode display.

Both the $\mathrm{x}$ and $\mathrm{y}$ axes have linear scales in a typical A-mode image. The $\mathrm{y}$-axis shows the received amplitude (or envelope) of the received signal; hence the name Amplitude-mode. The usage of a linear $y$-axis scale makes it difficult to determine small signal echoes, but easy to determine large echoes.

\section{Time Gain Compensation}

Time gain compensation (TGC) is often applied to A-mode images, and also to all other reflective ultrasound imaging modalities. TGC is motivated by the fact that as 
ultrasound propagates through a body, it is attenuated. Reflections from distant objects are therefore inherently weaker than reflections from (otherwise identical) closer objects. TGC is the process whereby all signals that take longer to return to a transducer are amplified, so that their magnitude becomes similar to the amplitude of those signals that returned to the transducer from closer objects. The amplification is typically applied in the manner of a smooth gain function - not as a step function.

In some systems, the TGC is automated - this is usually done by calculating the average signal level over a range and using this level as an indication of the gain required for that range.

\subsubsection{B-mode imaging (2D)}

B-mode (or brightness mode) imaging is a successor to A-mode imaging. Its motivation is to allow 2-dimensional images to be displayed, although it can be applied to both 1-dimensional and 2-dimensional images.

A 1-dimensional B-mode image would appear on a computer monitor as a single straight line of dots. Each dot would be grey, with the intensity of the dots being determined by the magnitude of reflected ultrasound over time. If the line was horizontal, then the $\mathrm{x}$-axis of the line would be the time axis - in an identical manner to the A-mode image in Figure 2.5. However B-mode images have no apparent yaxis as in the A-mode. Instead of plotting the received magnitude on the $y$-axis, it is plotted as the intensity (or greyscale value) of the line (or dots) at the corresponding point in time.

\section{Limitations of computer monitors (and the human eye)}

Modern computer monitors quantize the colours that they display - the colours are constructed from red, green, and blue components, and each of the red, green, and blue are quantized to 256 levels or shades - making $256^{3}$ (or 16.7 million) colours in all. Greyscales are shown as combinations of red, green, and blue in equal measures 
- so there are 256 available shades of grey. The limited number of available greyscale shades, makes B-mode inferior to A-mode in the case of 1-dimensional images.

It is unlikely that computer monitors will be able to display more colours in future, as it is generally accepted that the human eye can not differentiate any more than 256 shades of each of: red; green; and blue.

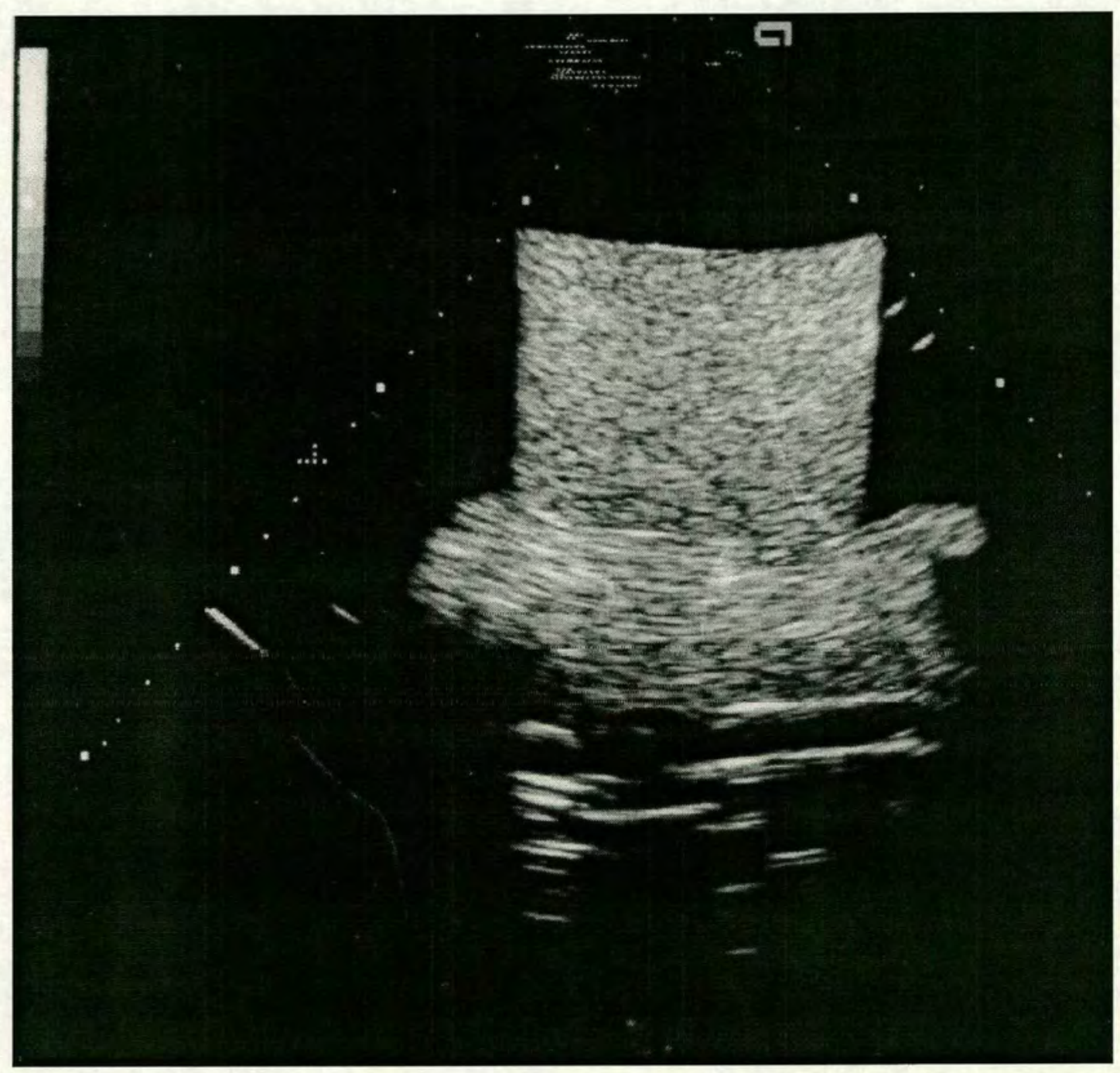

Figure 2.6 A typical B-mode, 2-dimensional image.

A typical B-mode image is shown in Figure 2.6. The object being imaged is a phantom, that is, a cubical block of tissue mimicking material. The material is jelly- 
like in nature. Note the diagonal lines of dots that define the perimeter of the region that is able to be imaged with this system. Note also the dark and light areas showing the contrast between the phantom and the surrounding water. The speckled pattern of the light areas is typical of B-mode medical ultrasound images.

In order to show greater detail in greyscale images, B-mode images are usually log transformed, and scaled so that full scale is between 30 and $60 \mathrm{~dB}$. This effectively amplifies small ultrasound signals so that become more clearly visible. e.g. at $50 \mathrm{~dB}$ full scale, a signal $25 \mathrm{~dB}$ smaller than the strongest signal will appear as $50 \%$ grey after log transforming. If the log transform was omitted, then a signal $25 \mathrm{~dB}$ below the strongest signal would appear as $5 \%$ grey (almost black and barely visible above the black background).

\section{$2 D$ image construction - in real time}

The advantage of B-mode imaging is that successive 1-dimensional images of neighbouring regions can be constructed into a composite image that is 2-dimensional (as in Figure 2.6). This scanning and image construction is able to be performed in real time, and it can appear to the user that the image is truly 2-dimensional.

Many commercial ultrasound systems have frame rates of 25 (or 30) frames per second. This means that every different 1-dimensional scan has been performed 25 times every second, and a new 2-dimensional image has also been constructed and displayed 25 times every second.

\section{$2 D$ image construction - transducer steering}

Between successive 1-dimensional scans, the transducer must somehow steer (or shift) so that a different part of the target image area is scanned. There are several common means of doing this, all of which are automated. The most important differentiating factor in these systems is whether the steering is done mechanically or electronically. 


\section{Mechanically steered transducers}

Mechanically steered transducers can be made with the transducer either rotating (through $360^{\circ}$ for a circular image or a lesser angle for a conical image), or translating (moving linearly back and forth orthogonal to the scan beam).

\section{Electronically steered transducers}

Electronic steered transducers can be made by having an array of transducer elements. The beam can then be steered either by activating only 1 element at a time (or a small group of neighbouring elements), or by activating all the elements but altering the phase or precise time at which each element is activated. The second approach has gained wide acceptance and is normally referred to as a phased array. Section 2.5 will discuss phased array systems in more detail.

\section{$2 D$ image construction - processing of raw RF ultrasound}

The processing that is performed between the scan and the image display is very important to any ultrasound imaging system. The signals are typically filtered, demodulated (or envelope detected), log transformed, and geometrically transformed [89].

\section{Demodulation}

In their raw form, the ultrasound signals are not suitable for display in either A-mode or B-mode. In A-mode the signals are processed by only one step - demodulation. Demodulation attempts to provide an envelope detection function. This is commonly performed with a Hilbert transform [90]. The Hilbert transform is defined as a filter whose transfer function is given in equation 2.4 : 


$$
\mathrm{H}_{\text {Hilbert }}(\omega)=\left\{\begin{array}{cc}
-j=e^{-j \pi / 2} & \omega>0 \\
+j=e^{+j \pi / 2} & \omega<0 \\
1 & \omega=0
\end{array}\right.
$$

The output of this filter is then called Hilbert[y(n)], and is the original signal with the phase of every Fourier component changed by $90^{\circ}$. Hilbert[y(n)] is defined in equation 2.5:

Hilbert $[y(n)]=\mathfrak{I}^{-1}\left\{H_{\text {Hilbert }}(\omega) \cdot \mathfrak{I}\{y(n)\}\right\}$

The final demodulated signal is obtained by applying equation 2.6 to the received signal $y(n)$.

$\mathrm{y}(n)_{\text {demodaduaced }}=\sqrt{\mathrm{y}(n)^{2}+(\text { Hilbert }[\mathrm{y}(n)])^{2}}$

Geometric correction and co-ordinate conversion using bilinear interpolation

Large numbers of transducers operate by rotating the ultrasound beam (either using rotation or a phased array), consequently it is often necessary to perform a geometric conversion (usually involving a conversion from polar to rectangular co-ordinates). The scan data often uses polar co-ordinates and all display monitors use a rectangular display grid. The co-ordinate conversion is typically performed using bilinear interpolation (see Appendix A).

\section{$2 D$ image quality}

There are multiple possible measurements of the quality of a medical ultrasound image. Clinical value is the most important measure in which the subjective assessment of contrast, texture, and boundary detection are important. However, 
objective measures also exist, and an important measure is the image resolution. The resolution must be specified in each dimension of the image, and is specified for a 2-dimensional image in Figure 2.7.

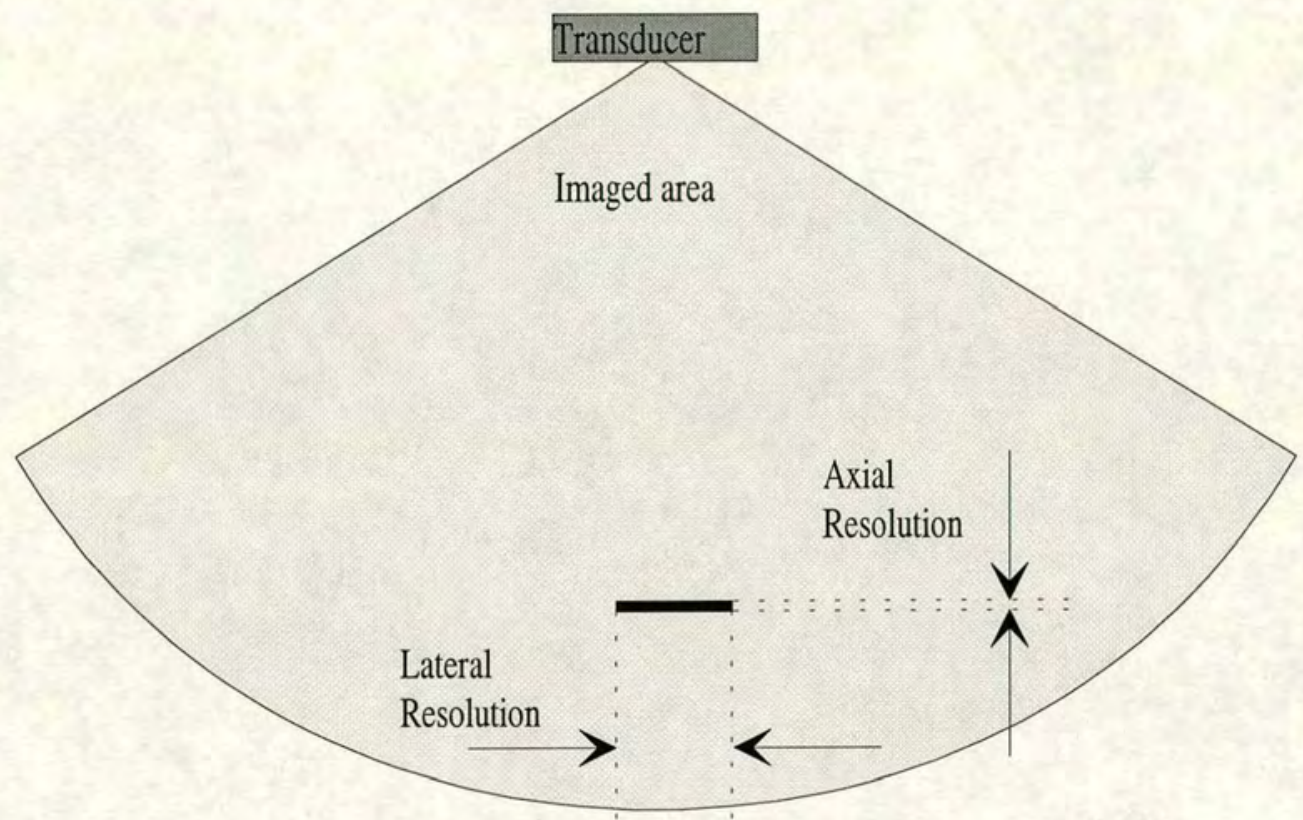

Figure 2.7 Definition of axial and lateral resolution for a $2 \mathrm{D}$ image.

The lateral resolution is limited by the width of the beam that is transmitted by the ultrasound transducer. This varies with distance from the transducer, but it is almost always larger than the axial resolution. This has implications for clinicians who can obtain more accurate geometric information by positioning the transducer to be perpendicular to surfaces of interest.

\section{Definition of axial resolution}

The resolution of an image (in any direction) is defined as the minimum distance by which two objects can be separated while remaining detectable as separate objects $[21,29]$. Therefore by definition, any two objects located closer together than the systems' resolution will appear in the image as being merged into a single object. 
For ultrasound images, the axial resolution is limited by the duration of the transmitted pulse. For reflective ultrasound systems it is equal to half the pulse duration multiplied by the speed of sound in the imaged object, as defined in equation 2.7:

Axial Resolution $=\frac{\tau \cdot c}{2}$

Where $c$ is the speed of sound in the medium, and $\tau$ is the duration of the transmitted ultrasound pulse. For transmission based ultrasound systems the axial resolution is given by equation 2.8 :

Axial Resolution $_{(\text {transmission based system) }}=\tau . c$

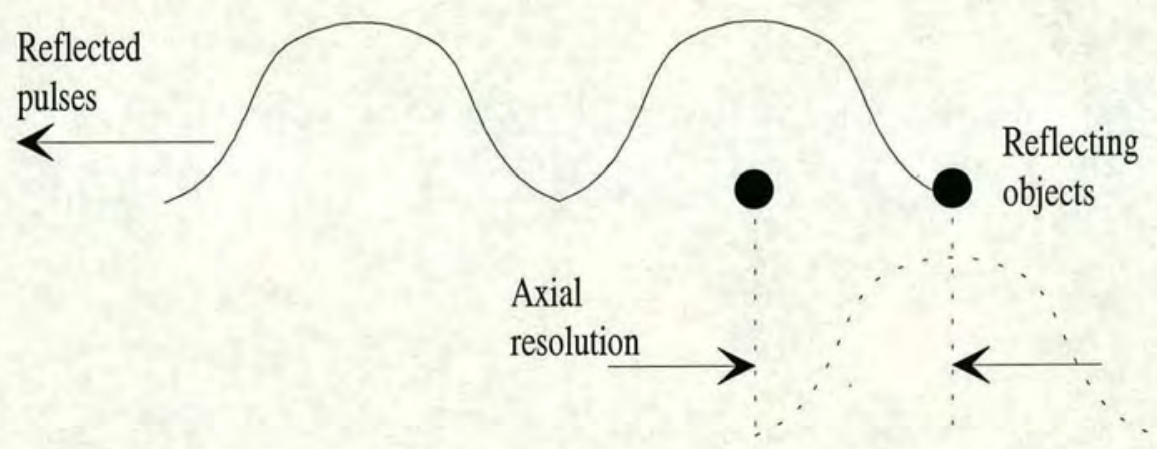

Figure 2.8 Two objects separated by the axial resolution

For the reflective ultrasound case, the axial resolution is illustrated in Figure 2.8 for two objects separated by the axial resolution of the system $(d=\tau \cdot c / 2)$. Here the source of ultrasound is on the left, and reaches the leftmost reflecting object first. The leftmost object reflects (part of) the ultrasound pulse, and after a further time $t=\tau / 2$ has elapsed, the rightmost object also reflects (part of) the ultrasound pulse. Due to the fact that the pulse reflected from the rightmost object has further to travel to the ultrasound receiver (the equivalent of an additional $t=\tau / 2$ time delay), the pulse from the rightmost object, will now reach the ultrasound receiver a time $\tau$ after 
the pulse from the leftmost object. Therefore the two pulses reaching the ultrasound receiver will (only just) not overlap; hence they are resolvable.

\subsubsection{M-mode imaging}

M-mode (or motion mode) images are 1-dimensional scans, that are scrolled across the screen over time (as a series of 1-dimensional B-mode images). The scrolling can be horizontal or vertical. The transducer will generally be held stationary, or near stationary, for this type of scan, and has a very high frame rate e.g. 600 frames per second (fps) [29]. M-mode traces are sometimes available on ultrasound imaging systems, usually as a secondary display mode.

This type of scan is useful for monitoring the motion of a particular anatomical object of interest. It is most often used in non-invasive coronary applications. Note that M-mode imaging is unrelated to intravascular ultrasound (IVUS), M-mode is used for monitoring heart movements, whereas IVUS is invasive and is used for monitoring arterial walls.

\subsubsection{Doppler imaging}

Doppler systems are used to measure movements in the body or object of interest. $[29,86,88]$ Often this is used in detecting and measuring foetal heartbeats and blood flow in major veins and arteries.

In order to obtain Doppler readings, a steady, single frequency, continuous wave must be used. Some systems used pulsed Doppler, but the transmitted signal is still long compared to other ultrasound imaging systems. The pulses must be long enough to stabilise, in order to measure the Doppler effects. The advantage of pulsing the Doppler signal is that depth measurements can also be taken.

Axial resolution in Doppler systems is inherently reduced due to the long duration of the transmitted ultrasound pulse. 
Doppler images are often presented in (false) colour where the different colours indicate magnitude (and direction) of movement (e.g. red and blue can indicate movements towards and away from the transducer respectively). Doppler scans can also be demodulated to the audio range, and observed aurally.

\subsubsection{D and 4D imaging}

An exciting development in ultrasonic imaging is the advent of 3-dimensional imaging, and the so called 4-dimensional imaging. 4-dimensional imaging is the name applied to real-time 3 -dimensional imaging (with time being the $4^{\text {th }}$ dimension).

There are currently no real-time 3D ultrasound imaging systems. Systems that are available physically move the transducer in order to scan the $3 \mathrm{D}$ volume (e.g. a TomTec machine used for data collection in this thesis rotated the transducer through up to $360^{\circ}$ in quantized steps while scanning $2 \mathrm{D}$ images at each step position). This movement is not able to be performed fast enough for the images to be acquired in real time.

There are two common techniques used for three dimensional visualisation (or rendering); surface rendering and volume rendering. These different approaches have very different requirements in how the ultrasound must be processed prior to being displayed. Recently new techniques have emerged, such as "semi-transparent volume rendering"[72], and "augmented reality"[73].

\section{Surface rendering}

This technique of 3D rendering involves defining a series of connected polygons that combine to form the complete shape of the object being rendered. In some graphics packages this lead to a rendering that appears chunky as a result of curved objects not being well suited to modelling with flat polygons. However newer packages attempt to simulate curved objects with curve fitting between the defined points of the 
polygons. This technique is generally successful and provides a realistic looking three dimensional image. An example is shown in Figure 2.9 which uses surface rendering.

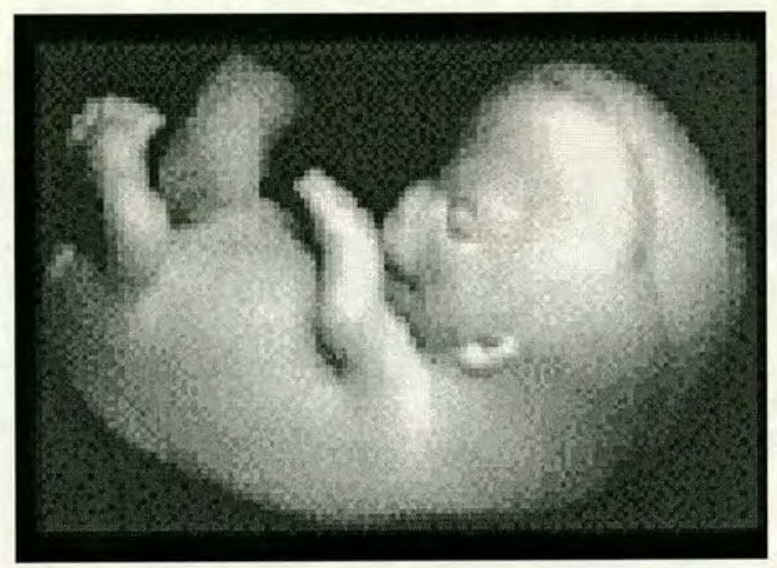

\section{Figure 2.9 Example 3D ultrasound image of a foetus}

The principal advantage of surface rendering of 3D images, is that it is computationally the most efficient known method. For this reason, many companies now produce video cards for IBM compatible PCs, that are optimised for surface rendering - allowing more complicated images to be displayed in real time.

One disadvantage of surface rendering is that the surface details can be lost if too few polygons are used. To avoid this, the number of polygons used may become huge which can become computationally expensive - thus image detail may be traded off against computational load.

A second disadvantage of surface rendering is that the image is presented solely in terms of surfaces, with no structural information available on the objects. Effectively this means every point in space has to be defined as either solid mass or empty space. For medical imaging applications this will result in loss of clinically useful information. This does not make the technique worthless, but it must be considered as an additional imaging mode, and not as a replacement for other imaging modes (such as B-mode). 
Finally, if surface rendering is to be used with ultrasound, then the edges of objects in ultrasound need to be extracted from the ultrasonic scans. Ideally this should be automatic. The accuracy of edge location is also an issue here, that will affect the final image accuracy, and thereby, the accuracy of diagnosis.

\section{Volume rendering}

This technique involves specifying the object to be rendered as a three dimensional set of "voxels" (a VOlume ELement; the 3D equivalent of a 2D pixel). In the simplest case, each voxel is specified as opaque or transparent (that is, as solid mass or empty space). However the technique is easily extended to defining each voxel as a grey level (corresponding to density), or as a colour [53, 76].

Volume rendering is superior to surface rendering for applications requiring tissue characterisation [72] (MRI and CAT and some ultrasound medical imaging systems use volume rendering). There are various methods for displaying a volume rendered image, with variations of ray-tracing being commonly used [74].

One drawback of volume rendering is that noise in the signal tends to be enhanced in the final 3D image. Different methods of ray-tracing attempt to reduce this problem, but this alters the image in other ways that are not always desirable (e.g. blurring).

\section{Semi-transparent volume rendering}

Semi-transparent volume rendering is a refinement of volume rendering proposed for usage in three dimensional medical imaging [73]. This idea proposes to make each volume "semi-transparent", so that although you can see the object, you can also see through it. This creates the possibility of observing structural details that would otherwise be more difficult to observe. This idea is similar to the view provided by $\mathrm{X}$-rays, which are familiar to medical personnel, as well as reasonably intuitive.

This system requires computation of the gradient of the image at each voxel, as well as the intensity of the image. The developers of this technique state that it requires 
randomly oriented $2 \mathrm{D}$ ultrasound images - if this is true, it would be difficult to collect sufficient scan data quickly enough to implement this technique in real time.

The computational demands of this system are not well documented however, and this may further restrict its suitability for real-time applications. Despite these drawbacks, it remains an exciting possibility for future research.

The extra details presented in the image could potentially cause confusion when analysed, suggesting that this technique would benefit significantly from tissue characterisation techniques such as pseudo colour enhancement.

\section{Augmented reality systems}

Another proposal for displaying ultrasound image visualisations is the idea of an augmented reality system [75]. This idea grew out of virtual reality systems, but differs in that the real world forms the basis of the display. This "reality" (usually seen through a video camera) is enhanced or augmented with the ultrasound image. The final image would then be (for example) a pregnant woman seen by a video camera, with a foetus image superimposed on this picture. This is an excellent method of obtaining precise spatial information, and is highly intuitive to use. Current implementations of this idea are nowhere near real-time. They also require manual specification of the region of interest.

\section{The advantages of 3-dimensional visualisations of medical images}

Three dimensional visualisations of medical images offer several advantages over two dimensional visualisations. These advantages include:

- reduced training time for medical personnel;

- extra reliability of diagnosis (abnormal features are easily visually detected);

- more detailed information about spatial relationships between objects; 
- the possibility of volume calculation (or other parameters), which could be automated, giving accurate diagnoses of quantities such as foetal growth.

MRI and CAT analysis already use three dimensional visualisation of medical images. These signals are however easier to work with than ultrasound due to their good signal definition, and low noise content.

\section{Real time rendering of $3 D$ images}

Computer hardware (and software) is emerging that increases the complexity of 3D images that can be rendered in real time. Most 3D software uses the surface rendering technique. The user must specify the shape and layout of each surface.

Real time medical ultrasonic imaging requires that the shape be determined from the ultrasound signals immediately before being rendered. This information is usually obtained by taking a series of 2D images, with a known separation (and angle) between each image. The real-time rendering system must combine the $2 \mathrm{D}$ images to construct the three dimensional image.

\section{Challenges in $3 D$ ultrasound imaging}

Since surface rendering is computationally much more efficient than volume rendering, it is likely that surface rendering will be more feasible than volume rendering - at least in the short to medium term [76].

In surface rendering of medical ultrasound images there is the significant difficulty of extracting the location of the edges automatically (or even manually) from ultrasound. This is due to ultrasound's poor signal to noise ratio and poor dynamic range. Errors may arise in their precise location, and also in their existence or not. These errors would cause inaccuracies in clinical measurements such as foetal volume. Some researchers are currently working on this problem [26, 69, 70, 91]. They have proposed techniques for detecting edges including using a simple intensity threshold (usually after time gain compensation), and using the image gradient 
(where a high gradient indicates a likely edge [71, 72, 77]. However, accurate and reliable edge extraction from ultrasound remains a difficult problem.

The difficulty of data acquisition time (the time required to scan a given volume) is large with conventional transducers which scan 1D at a time. Some researchers have proposed transducer systems that will scan images more quickly by simultaneously scanning 2D images [59, 61, 78, 79].

\section{Example 3D ultrasound images}

Figure 2.10 shows an artery - scanned from the inside but viewed from the outside. This is an IVUS $3 \mathrm{D}$ image, built up with a rotating scanner, that is repeatedly pulled back by a stepping motor.

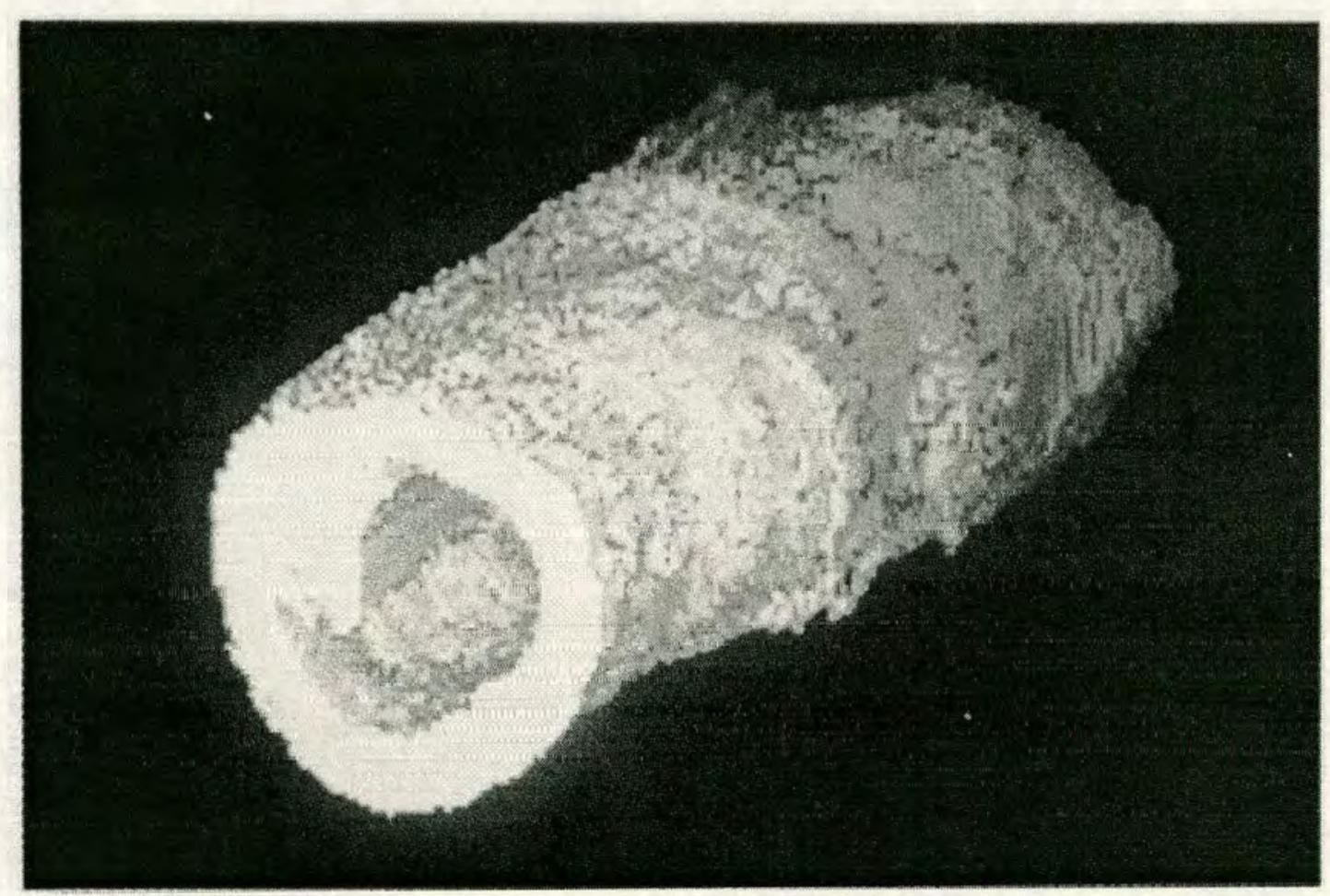

Figure 2.10 An artery in 3D. (from Cavaye and White [12]).

Figure 2.11 and Figure 2.12 are examples of foetal 3D images. This is a common area of 3D image applications, as foetal shape abnormalities are of clinical interest, 
as is foetal volume (for growth estimation). These images were constructed by Dr. B Benoit using surface based rendering.

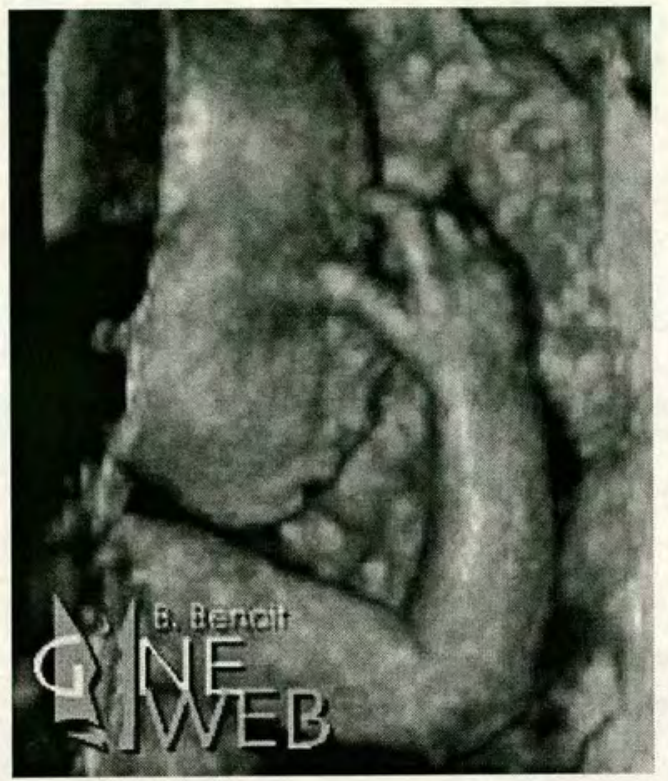

Figure 2.11 A foetal face and arm in 3D. (from Dr. B Benoit)*.

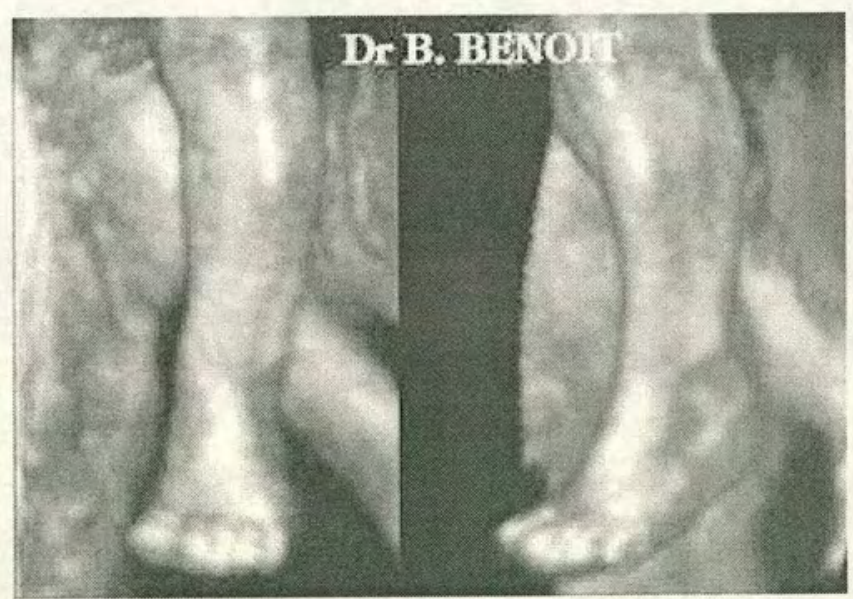

Figure 2.12 A foetal leg in $3 D$ from two angles. (from Dr. B Benoit) ${ }^{*}$

* Published on Dr. B. Benoit's WWW site: http://www.gyneweb.fr/bbenoit/Images3D/Images3D.html. This site was valid at February 1998. 


\subsection{Phased array ultrasound systems}

A phased array is constructed as an array of piezoelectric crystals. These crystals each receive an excitation voltage pulse at (almost) the same time. The slight variation in the excitation times cause the beam to be steered and also focused [92].

\subsubsection{Phased array transmission}

If the piezoelectric crystal elements were all excited simultaneously then the situation in Figure 2.13 results.

The beam of ultrasound can be steered to the left or right by any chosen angle by adjusting the excitation times in a delayed manner from left to right (or right to left) as in Figure 2.14. Note that the wavefront and the beam direction are perpendicular.

The beam can be focussed by adjusting the excitation times to have a certain "curvature" of arrival time as indicated in Figure 2.15. The principles of focussing and steering can be used together to produce a steered and focussed beam of ultrasound.
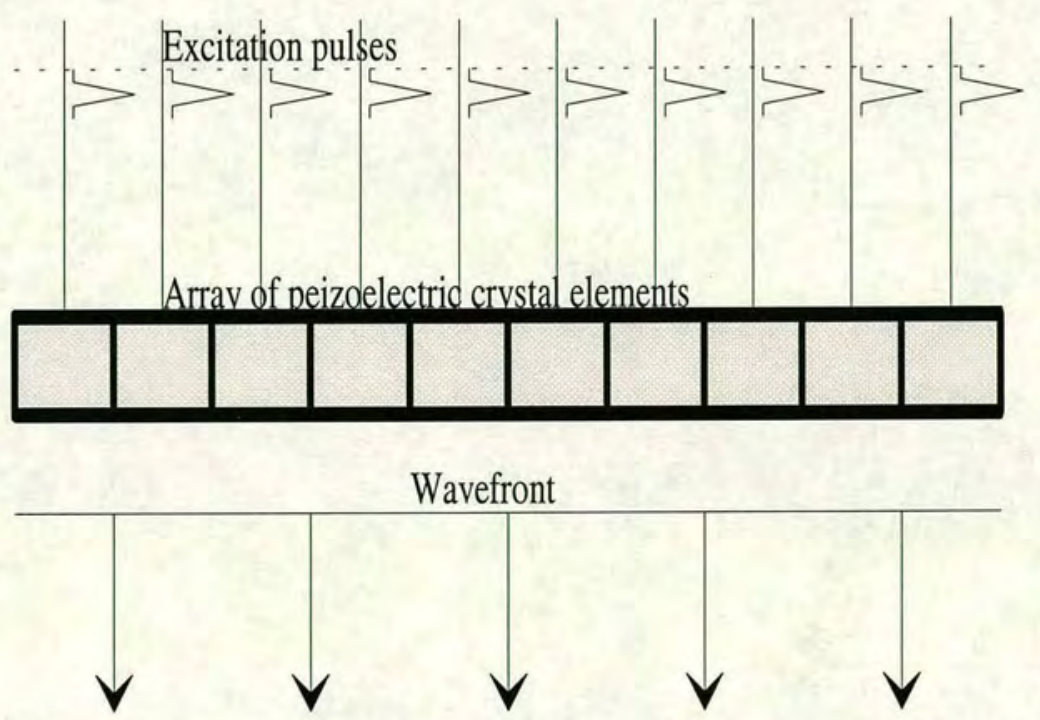

Figure 2.13 Simultaneous excitation of phased array elements. 

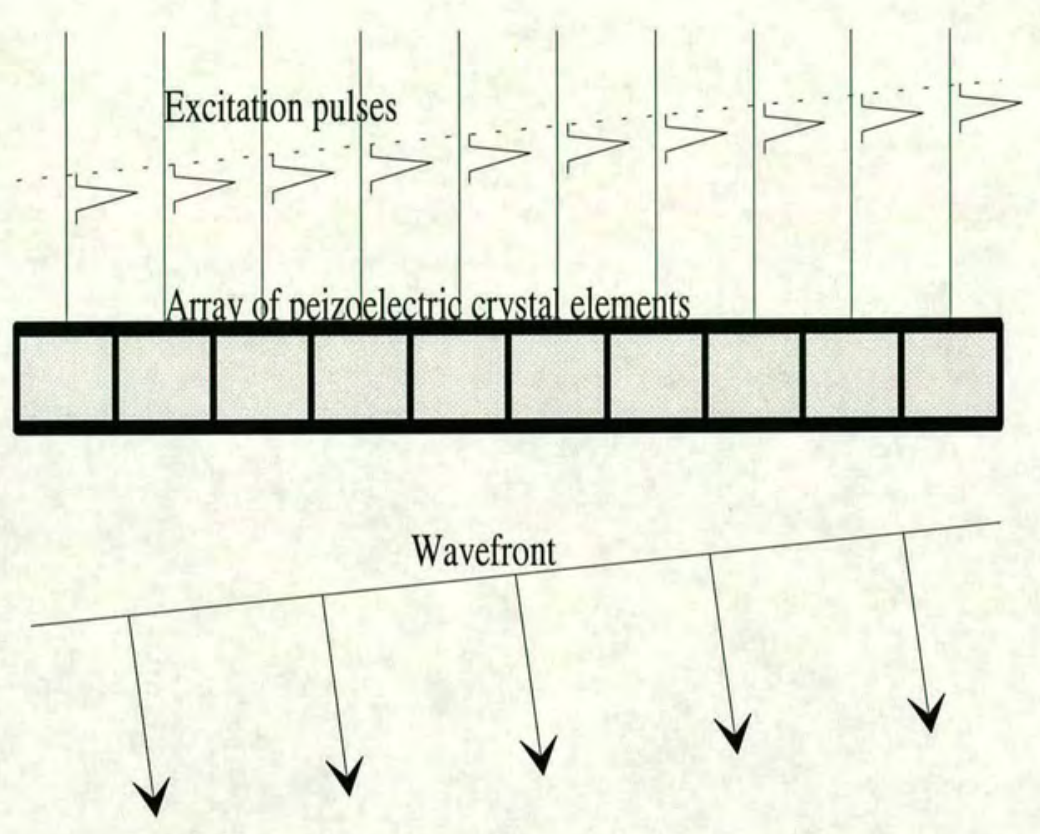

Figure 2.14 Beam steering for a phased array.
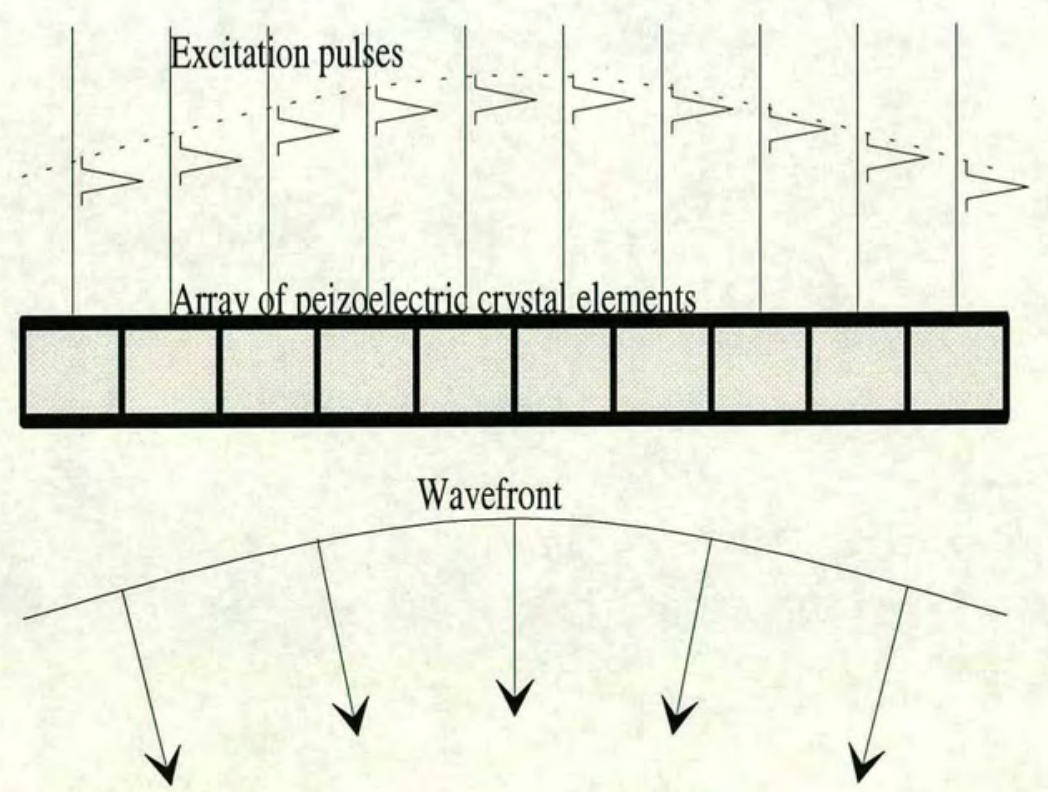

Figure 2.15 Beam focussing for a phased array. 


\subsubsection{Phased array receiving}

Just as an array can have its signals phased (or delayed) during transmission, so it is also possible to perform a similar operation on receive. This is done during the summation process. The simplest form of summation is the receive equivalent of Figure 2.13 - where every element is simply summed with no delays between elements.

In order to highlight the beam or signal coming from a certain direction, the receive equivalent of Figure 2.14 can be used (i.e. delayed summation).

In order to focus the reception at a particular distance, the receive equivalent of Figure 2.15 can be used. This technique turns out to be very powerful, since for pulse-echo scan, the focus depth can be changed dynamically as the beam propagates. In this way, the system can (to a degree) be focussed at all depths. The improvement in image quality resulting from this technique is significant.

\subsubsection{2-Dimensional phased arrays}

In the above sections, a phased array was considered in one dimension only. This is indeed a common implementation and it is widely used in medical imaging. It is however possible to have a 2-dimensional array. This has two principal advantages:

- the beam can be focussed in two dimensions;

- the beam can be steered in two dimensions (only if the grid array in Figure 2.16a is used; this is not possible for the concentric rings array in Figure 2.16b).

2-dimensional arrays are operated in much the same manner as a 1-dimensional array, except that the each element will be delayed by steering and focussing from two dimensions. The delays from each dimension sum together just as the steering and focussing delays sum together in the 1D case. 


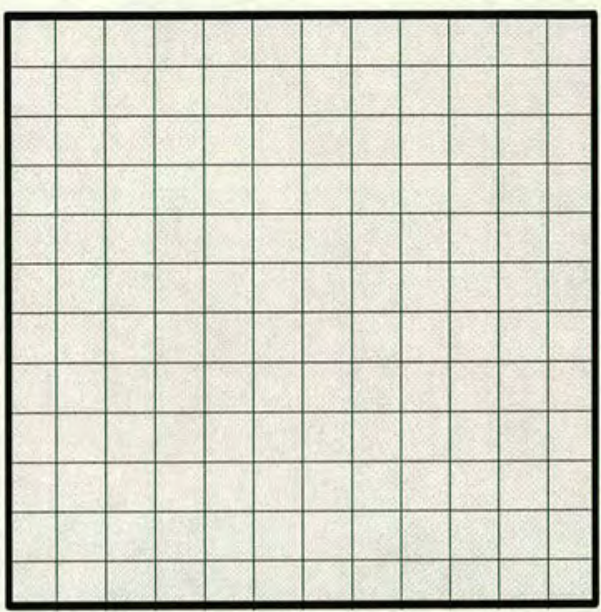

(a)

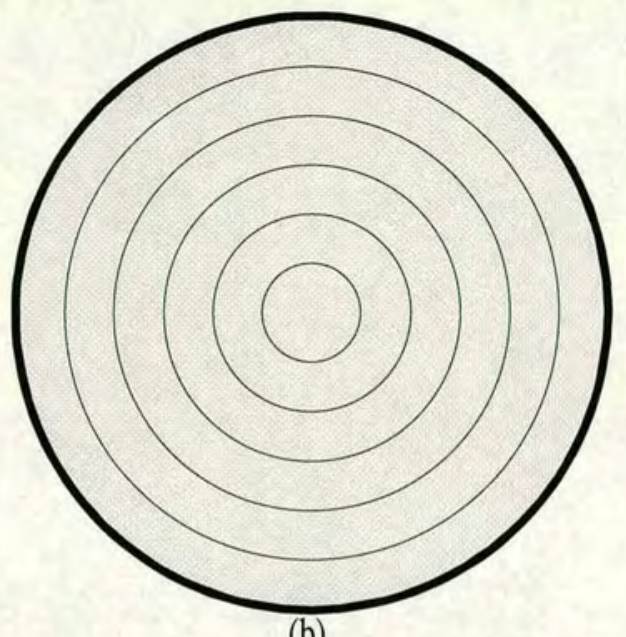

(b)

Figure 2.16 Example 2D array structures.

2-dimensional arrays are the subject of current research into 3D ultrasonic imaging. The motivation for this is that a $2 \mathrm{D}$ grid array can be steered electronically to make a conical imaging volume. This will remove the need for mechanical movements, which will make the system easier to operate - this has been shown to be of great clinical benefit (when 2D B-mode imaging replaced 1D A-mode imaging).

\subsubsection{Single element ultrasound systems}

The alternative system of transducer manufacture and operation is to use only a single element. Single element systems have limitations compared to phased array systems. The beam cannot be steered, and the focus is fixed - controlled only by the geometry of the transducer. In order to generate $2 \mathrm{D}$ or $3 \mathrm{D}$ images, the transducer must be physically moved (translated, rotated, or both). This difficulty means that single element transducers are not widely used in non-invasive ultrasound. They are however still widely used in intravascular ultrasound where the transducer is required to be physically small. 


\subsection{IVUS imaging}

Intravascular ultrasound (IVUS) is an invasive medical imaging technique where a catheter containing an ultrasound transducer is inserted into the subjects artery [93]. The generalised system is shown in Figure 2.17. IVUS is used to obtain information about the condition of the subjects arteries such as atherosclerosis, occlusions, partial occlusions, plaque formations, thrombosis, and arterial thickening in general.

The advantage of using an invasive technique is that the ultrasound has less distance to travel, therefore higher frequencies can be used, allowing a higher image resolution. Invasive imaging also removes other difficulties such as bone or air obstructions that can hinder non-invasive ultrasound imaging - especially beneath the ribcage. IVUS is the only available technique that allows clinicians to see inside arteries, for this reason it is commonly used.

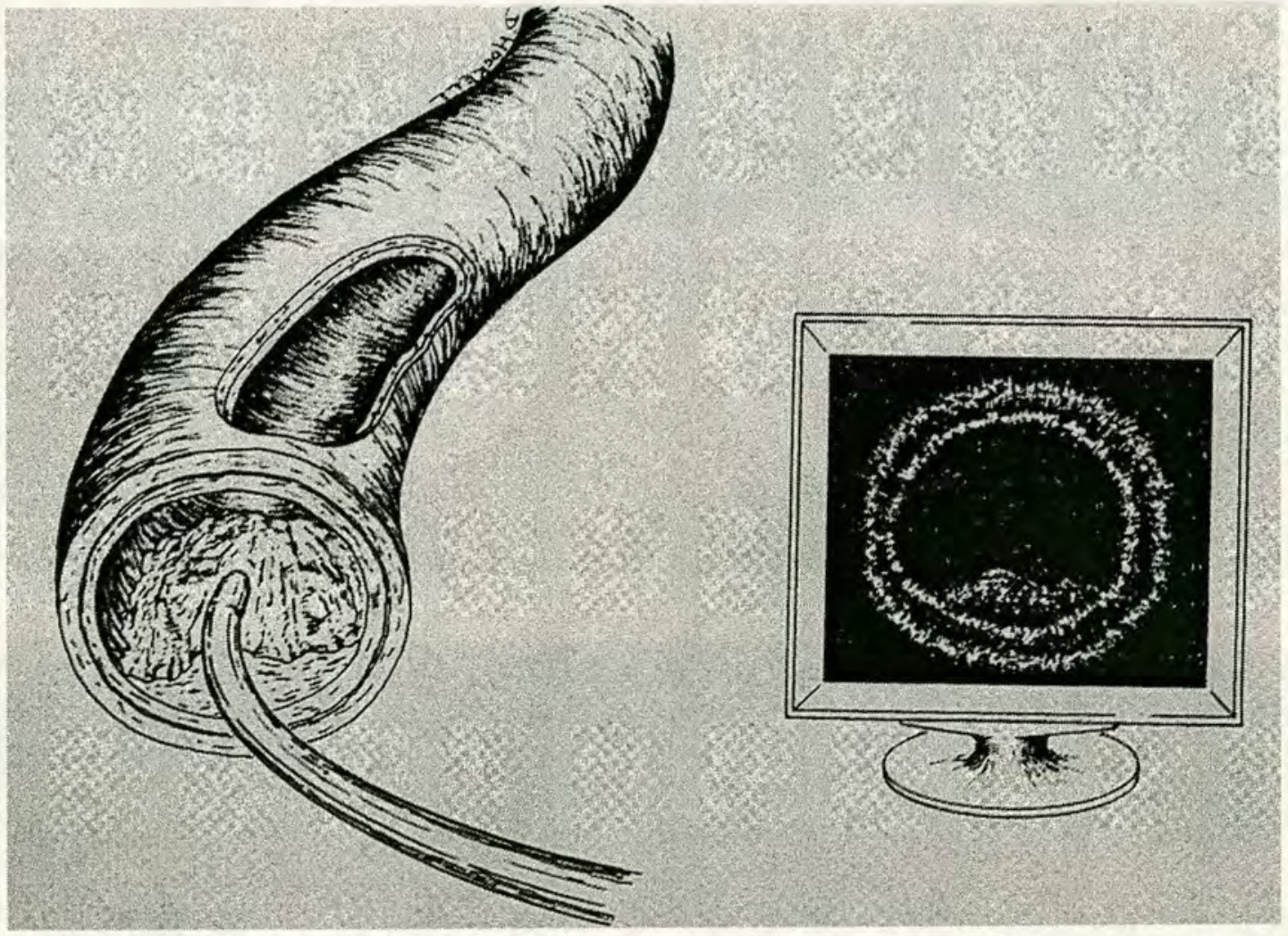

Figure 2.17 An IVUS catheter and arterial imaging system. (from Cavaye and White [12]). 


\subsubsection{IVUS catheters}

IVUS transducers are usually single element transducers. The motivation for this is that the catheters need to be small in order to fit into the arteries. When IVUS imaging of an artery is required, it is often because the artery is blocked or occluded (or partially obstructed). Therefore, a smaller catheter will be able to travel through smaller gaps in the artery and obtain more clinically useful information.

Because IVUS transducers are single element, they cannot steer electronically, therefore they must steer by mechanical movement if the image is to be more than 1D. Typically, IVUS transducers will have two components of mechanical movement (rotation and translation).

The transducer will rotate through $360^{\circ}$, obtaining a series of $1 \mathrm{D}$ images at each position as it rotates. These $1 \mathrm{D}$ images will then be used to construct a $2 \mathrm{D}$ (B-mode) image. The imaged area will be circular, and perpendicular to the direction of the artery (as a first approximation). To be more accurate, most transducers are slightly forward looking, - so the beam actually points forward of perpendicular by a few degrees. This is shown in Figure 2.18. The makes the imaged area actually a (hollow) cone rather than a (planar) circle. Some IVUS transducers are built to point directly forward.

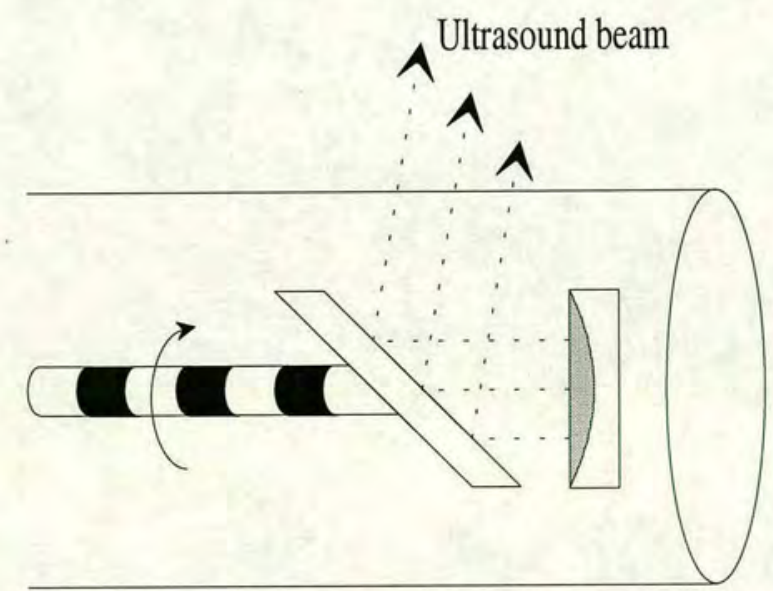

Figure 2.18 A typical IVUS catheter-slightly forward looking. 
After obtaining the first 2D image, the transducer will be "pulled back" by a small distance (using a stepping motor), and a second 2D image will be obtained. Successive "pullbacks" and 2D image acquisitions are performed, giving a full picture of the artery. The catheter can also be pulled back by hand. Although this will not produce evenly spaced $2 \mathrm{D}$ images, it can be useful for coarse positioning or locating regions of interest.

The successive 2D images can be collated to form a 3D image of the artery. Systems that perform this type of function do not operate in real time. This is due not only to the large amount of computer processing that is required, but also to the lengthy time of acquisition for the full 3D dataset.

\subsubsection{Typical IVUS images}

Two IVUS images are shown in Figure 2.19. These images were obtained with very high frequency ultrasound $(40 \mathrm{MHz})$ giving them a superior resolution to many other IVUS images. Visible are the internal elastic lamina (i), media (m), and adventitia (a), plus some intimal thickening ( $t$ ), and plaque formation (p). The three ringed image (light-dark-light) is characteristic of muscular arteries. Figure 2.20 gives some explanation as to the features that are shown in the IVUS images of Figure 2.19. 


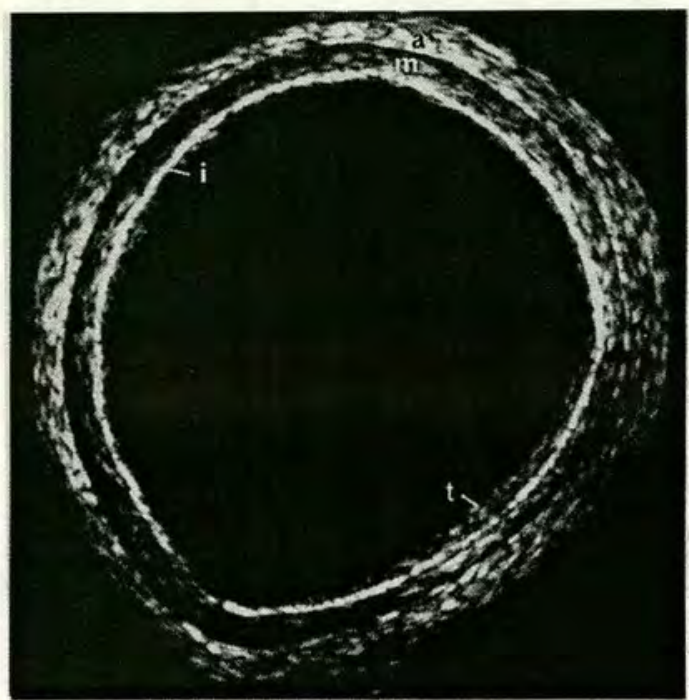

(a)

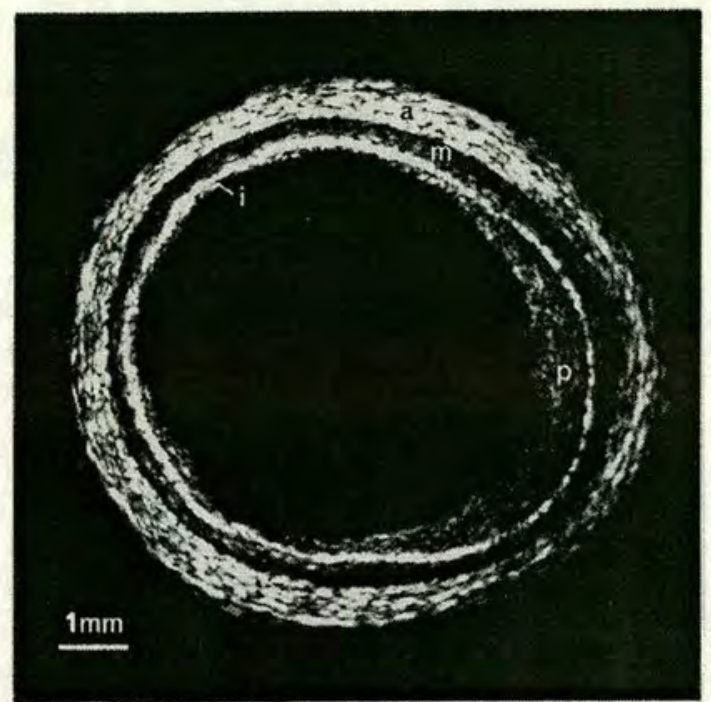

(b)

Figure 2.19 Example IVUS images. (from Cavaye and White [12]).

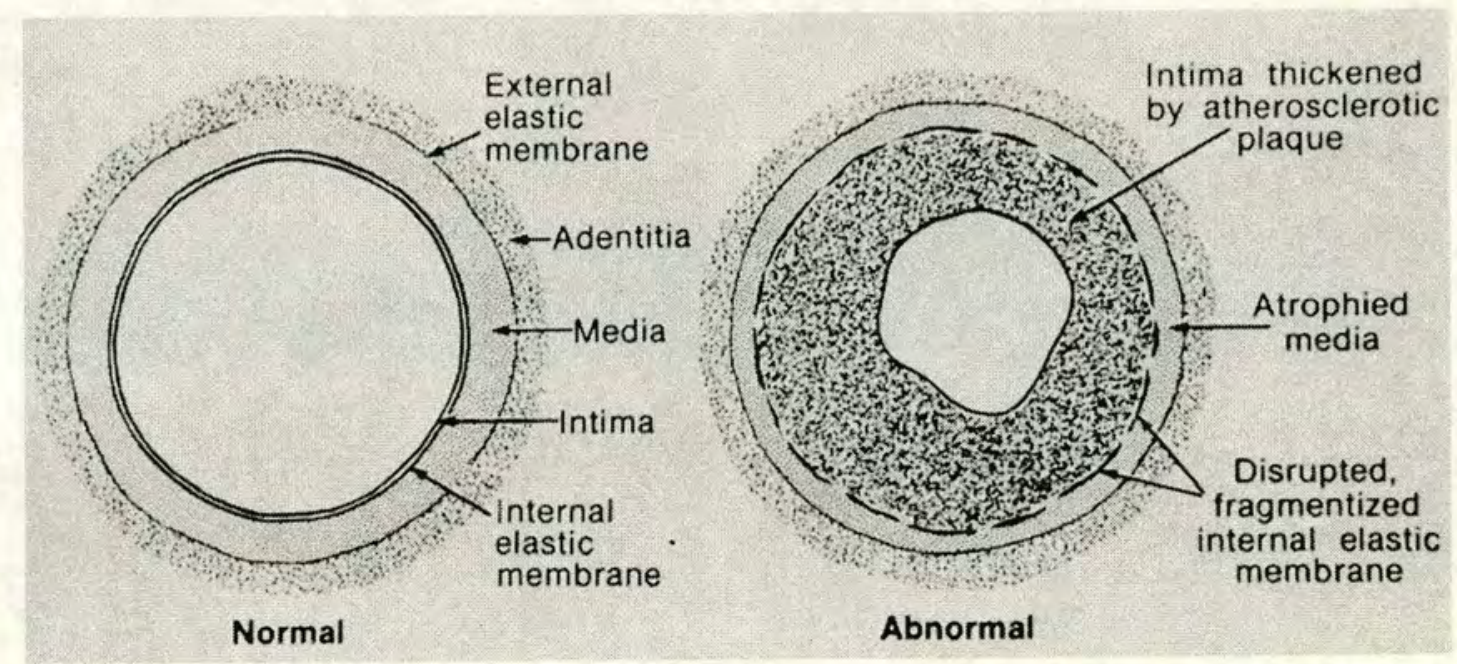

Figure 2.20 The structure and some features of arteries. (from Tobis and Yock[94]).

Another example IVUS image is shown in Figure 2.21. This image is from the Aorta of a rabbit.

The corners of Figure 2.21 do not contain any information - they are filled in only to make the image square to facilitate storage in a computer bitmap file. 


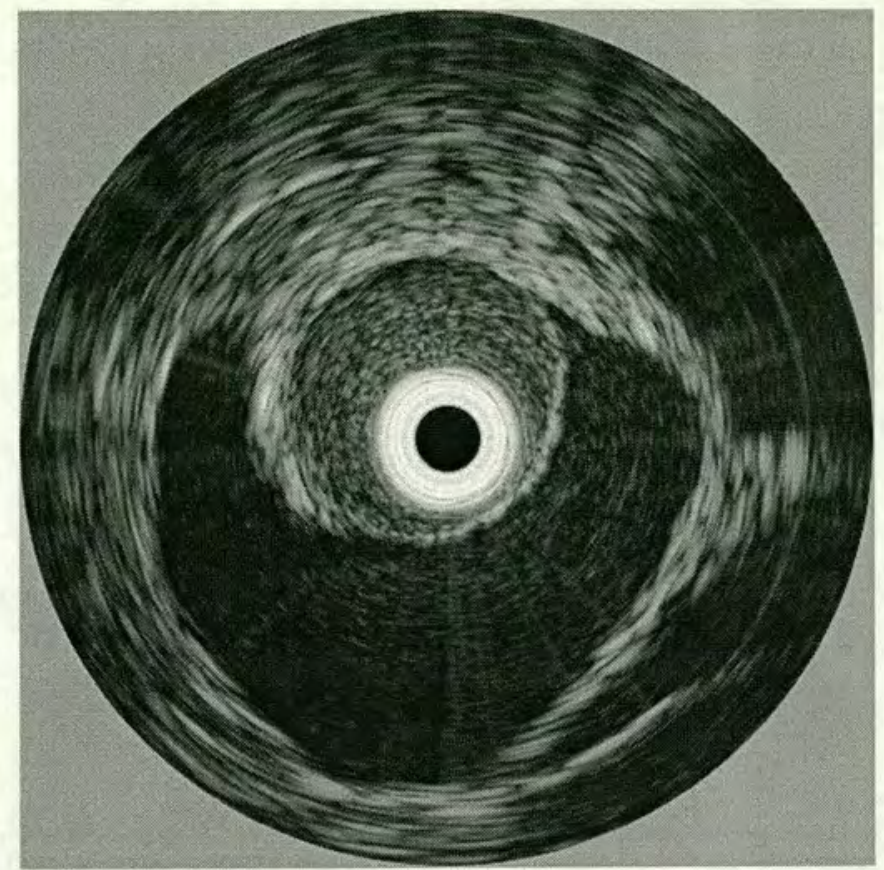

Figure 2.21 Example IVUS image with ringdown and a diverging artery.

In the centre of Figure 2.21, the black region is artificially imposed into the image, and contains no information. This region represents the area physically occupied by the transducer, that was unable to be imaged due to the presence of the transducer itself.

The bright region surrounding the black catheter region is called the "ringdown" region. This is signal that is detected by the transducer that has resulted from reverberations within the transducer itself. This ringdown can be removed from the displayed image when the image depth is sufficiently large in relation to the ringdown, and there is expected to be no clinically useful information within the ringdown region (such as in obstetric ultrasound). The ringdown is usually retained in IVUS imaging due to the artery boundaries sometimes occurring inside the ringdown region (or very close to it).

The clinically useful information in an IVUS image is contained between the ringdown and the outer limit of the image. In this region, clinicians look for the location of edges within the image which result from tissue boundaries. This is 
sometimes presented as a 3D image, which aids a clinician's understanding of the geometry, shapes, sizes and locations of each component part of the artery.

Also, there is the textural information contained within each region (between boundaries). This textural information may indicate to a clinician what type of tissue is present. Some researchers have attempted to determine tissue type automatically from the image $[21,22]$ with some degree of success.

\subsection{Conclusion}

Ultrasound imaging is widely used in medicine, despite producing images that are inferior to other medical imaging techniques.

The poor image quality of ultrasound presents many challenges in interpretation and presentation of the images. This is especially true in 3D images - whether using surface based rendering with edge extraction, or volume rendering where noise is often enhanced. Noise suppression techniques have been tried, but many suffer from the fact that they remove useful texture information from the image (e.g. median filtering) [42,95], or other problems such as blurring (e.g. a matched filter).

In 2D ultrasound images (and 3D), the system resolution remains poor compared to other imaging techniques. Improving the system resolution would improve the usefulness of ultrasound in medical imaging.

Automated edge extraction techniques are the subject of ongoing research [96] in both 2D and 3D (the same techniques are often used for both 2D and 3D). 


\section{Chapter 3.}

\section{FILTERING}

\subsection{Introduction}

Filters are typically used to extract a signal from background noise. This usually increases the signal to noise ratio, and allows the signal to be interpreted or measured with greater accuracy.

The simplest types of filters will pass a chosen bandwidth of signals, while blocking or attenuating any signals outside of the chosen bandwidth. The filter will typically be designed so that the attenuation affects the noise more than the signal.

In the simplest case, noise can be presumed to be additive, white and Gaussian (AWGN). Most filters are designed using an assumption that the noise present is AWGN. In some cases the noise can be non-white, deterministic, or multiplicative. The type of noise can affect the success of a filter, and so different filter designs have been developed - each designed for a certain application or situation.

Filters are always designed in order to form part of a system, and their design is thereby affected by the characteristics of the other components of the system. In the simplified case, we often have the situation shown in Figure 3.1 where a signal passes through a distorting channel, and is altered. The addition of noise makes it impossible to reconstruct completely (and perfectly) the original signal. Although the affect of the channel can be partially reversed or removed by a filter, it can be fully removed only in the absence of noise. We can surmise that the signal reconstruction by means of a filter (see Figure 3.2) will be more accurate if the noise level is low relative to the signal. 


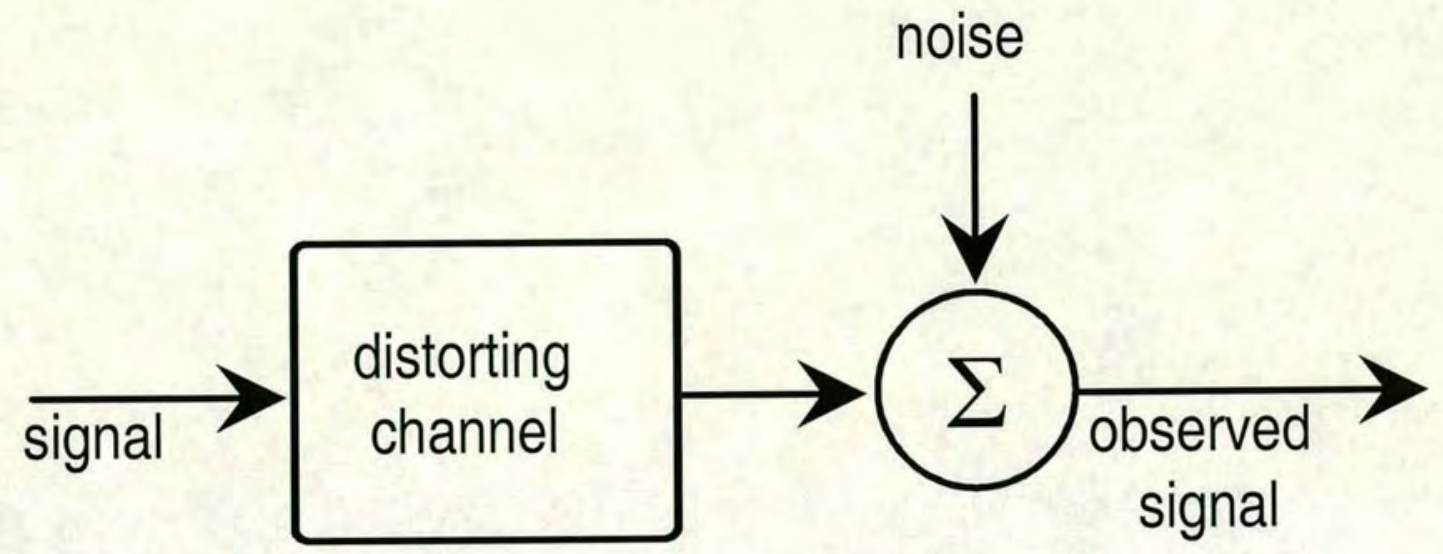

Figure 3.1 Typical system to which a filter would be applied.

If the input signal to the distorting system or channel is a perfect impulse (a dirac delta function) then the observed sequence will be the system impulse response $\mathrm{h}(n)$. The Fourier transform of the system impulse response is the system transfer function $\mathrm{H}(\omega)$.

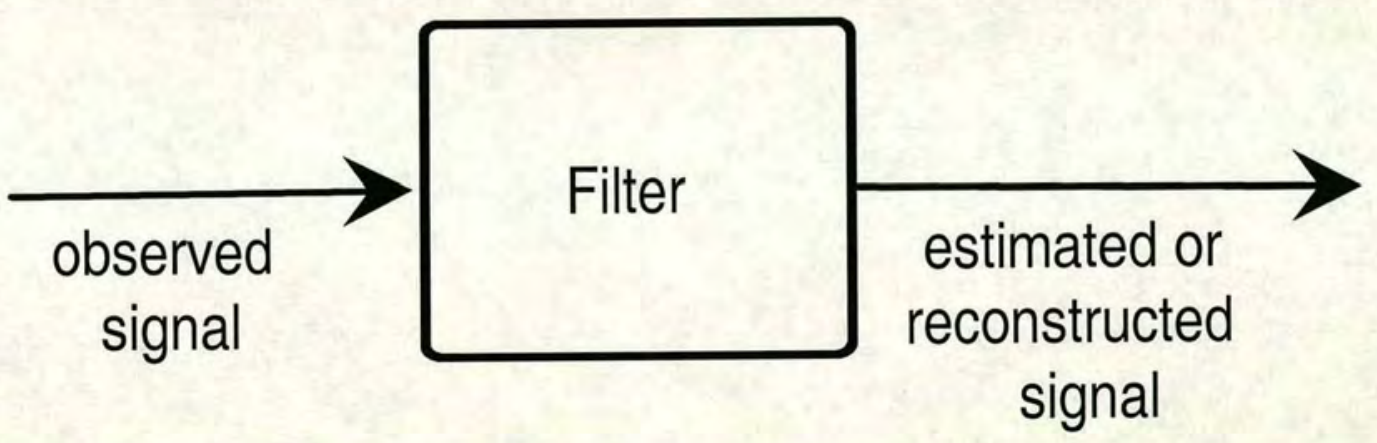

Figure 3.2 A filter can be used to estimate or reconstruct the original signal.

The details of the filter in Figure 3.2 are at the discretion of the system designer. Filters can be classified as linear or non-linear, adaptive or time-invariant, and as finite impulse response (FIR) or infinite impulse response (IIR).

No form of adaptive filters are covered here, because they have not been applied to any form of ultrasonic imaging. 


\subsubsection{The assumed model for filter design}

For this chapter the model given in Figure 3.3 will be used. This shows the relationships between definitions of $\mathrm{h}(n), \mathrm{x}(n), \mathrm{y}(n), \mathrm{H}(\omega), \mathrm{X}(\omega)$, and $\mathrm{Y}(\omega)$ within the system model. Where $\mathrm{H}(\omega), \mathrm{X}(\omega)$, and $\mathrm{Y}(\omega)$ are the Fourier transforms of $\mathrm{h}(n), \mathrm{x}(n)$, and $\mathrm{y}(n)$ respectively.

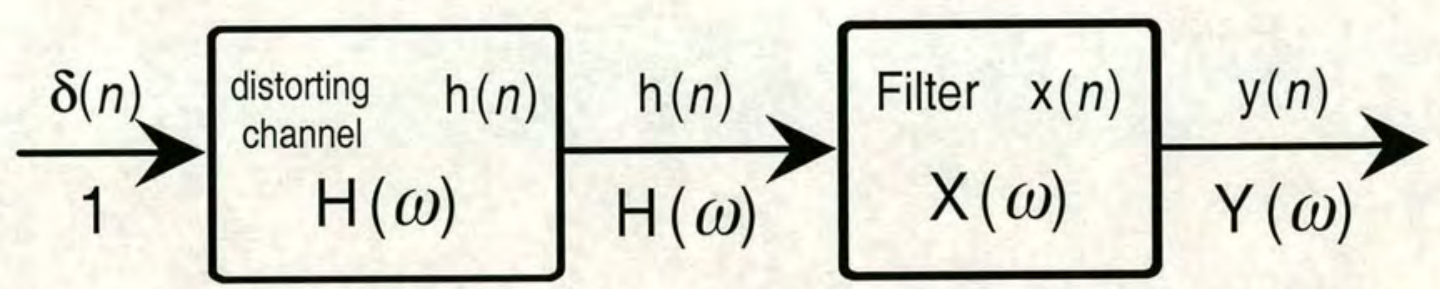

Figure 3.3 The assumed model for filter design.

The estimated reconstructed signal - in the absence of noise and with a perfect impulse $\delta(n)$ as the initial system input - is $\mathrm{y}(n)$. $\mathrm{y}(n)$ is defined in equation 3.1 as the convolution of $\mathrm{x}(n)$ and $\mathrm{h}(n)$. Note that this is only true when the channel $\mathrm{H}(\omega)$ is linear and time-invariant. The frequency domain equivalent of $\mathrm{y}(n)$ is $\mathrm{Y}(\omega)$, which is defined in equation 3.2:

$\mathrm{y}(n)=\mathrm{x}(n) * \mathrm{~h}(n)$

$\mathrm{Y}(\omega)=\mathrm{X}(\omega) \cdot \mathrm{H}(\omega)$

This chapter contains a review of some different filter designs. Many of these designs have been proposed as being useful for filtering medical ultrasound. The example designs for each type of filter are based on an example measurement of an observed signal, which will be assumed (for this chapter) to be the result of applying a dirac delta function (a perfect spike or impulse) at the input to the distorting channel. This means that the observed signal is equal to the system impulse response $\mathrm{h}(n)$. The 
distorting channel used is a medical IVUS imaging system, with the signal reflected by a near perfect plane reflector.

\subsection{Matched filter}

A matched filter is designed to maximise the signal to noise ratio, for a system where the noise level, and the channel or system is stationary, and where the noise is additive, white and Gaussian. The matched filter for a system is designed by time reversing the (digitised) system impulse response. In the frequency domain this yields a filter whose frequency response (in magnitude) is equal to the frequency response of the system - but has its phase conjugated. Mathematically this is expressed in equation 3.3 (for the time domain) and also in equation 3.4 (for the frequency domain):

$$
\mathrm{x}(n)=\mathrm{h}(-n)
$$

$\mathrm{X}(\omega)=\mathrm{H}^{*}(\omega)=\operatorname{Arg}\left\{\mathrm{H}^{*}(\omega)\right\} \cdot|\mathrm{H}(\omega)|$

where $H(\omega)$ is the system transfer function. This is equal to the Fourier transform of the impulse response vector $\mathrm{h}(n) \mathrm{X}(\omega)$ is the frequency response of the filter.

While the matched filter will maximise the signal to noise ratio of a system, it also produces a spreading effect on the system's impulse response. Thus a matched filter causes a effective decrease in the time resolution of the signal. For this reason the matched filter is not the optimal filter for enhancing the resolution of a system.

Since in the frequency domain, the filter's phase response is the conjugate of the phase response of the system, it follows that after any signal passes through the system and its matched filter, the phase of every Fourier component will be the same as the original signal. This phase alignment is a characteristic shared with many other filters reviewed here. 


\subsubsection{Example design of a matched filter}

Figure 3.4 (left) shows the impulse response of an IVUS imaging system. In order to design a matched filter for this system, we must time reverse the impulse response. This is shown in Figure 3.4 (right). The digitised samples of Figure 3.4 (right) then become the filter taps for the matched filter. (For any digital FIR filter, the filter taps are equal to the filter's impulse response).
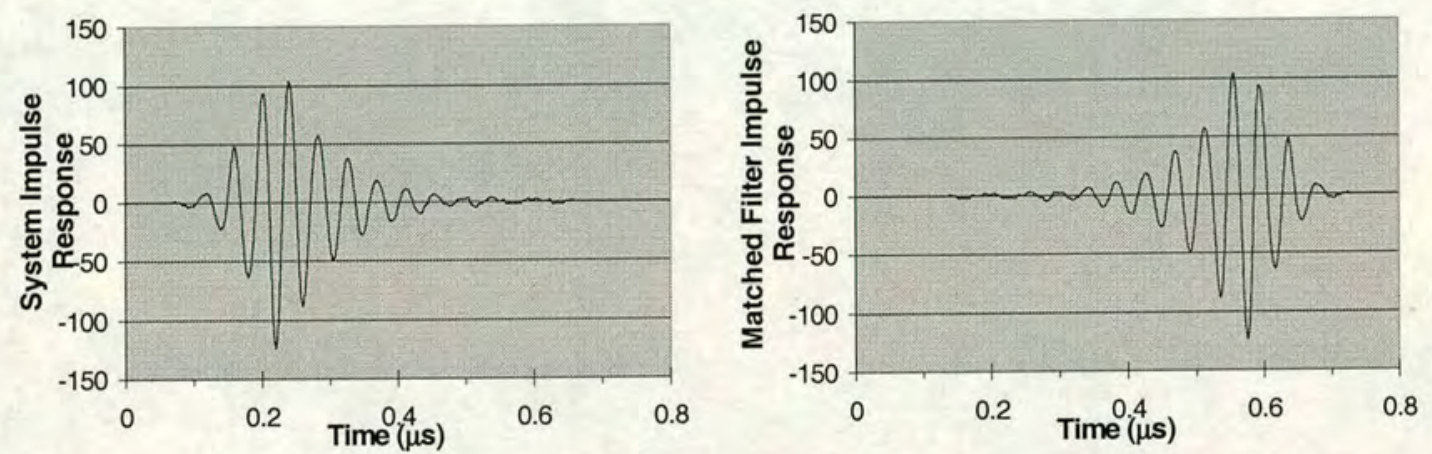

Figure 3.4 Matched filter design (right) by time reversal of system impulse response (left).

Note that in Figure 3.4 (left and right) the y-axes have arbitrary units. The $x$-axis is plotted against time, but could equally well be plotted as discrete digitised sample points. Figure 3.5 shows the relationship between the system impulse response and the corresponding matched filter response in the frequency domain - that is, they are equal in magnitude (the phase of each Fourier component is negated).
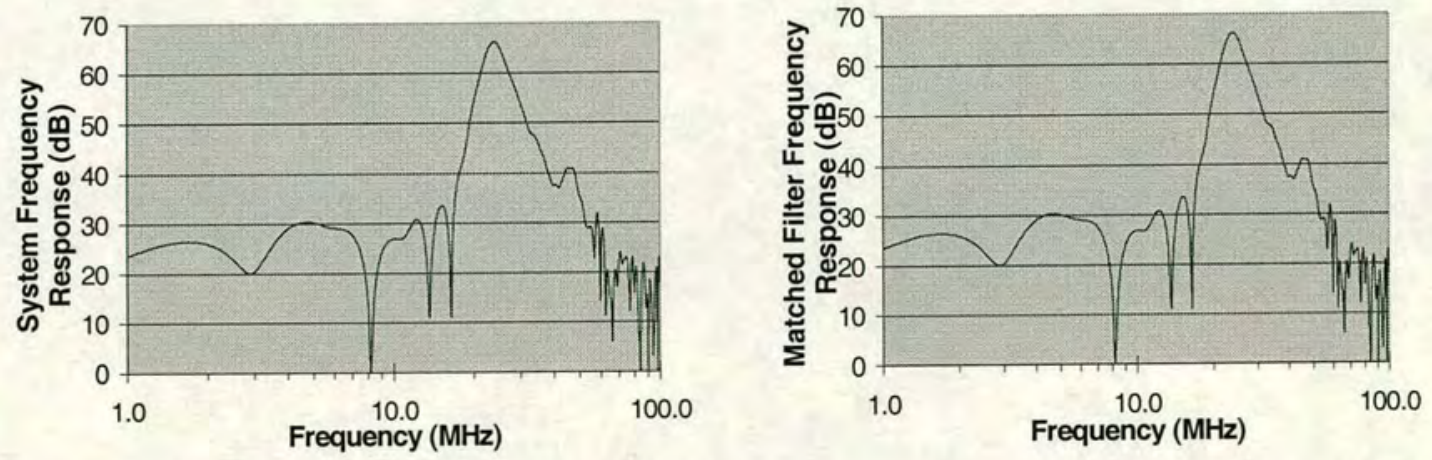

Figure 3.5 Magnitude response of the system impulse response (left), and the matched filter design (right) 


\subsubsection{Example application of a matched filter}

Note that in Figure 3.6, the example IVUS image in Figure 3.6 (left) has been blurred significantly after application of a matched filter (right). This causes a loss of detailed information in the image.
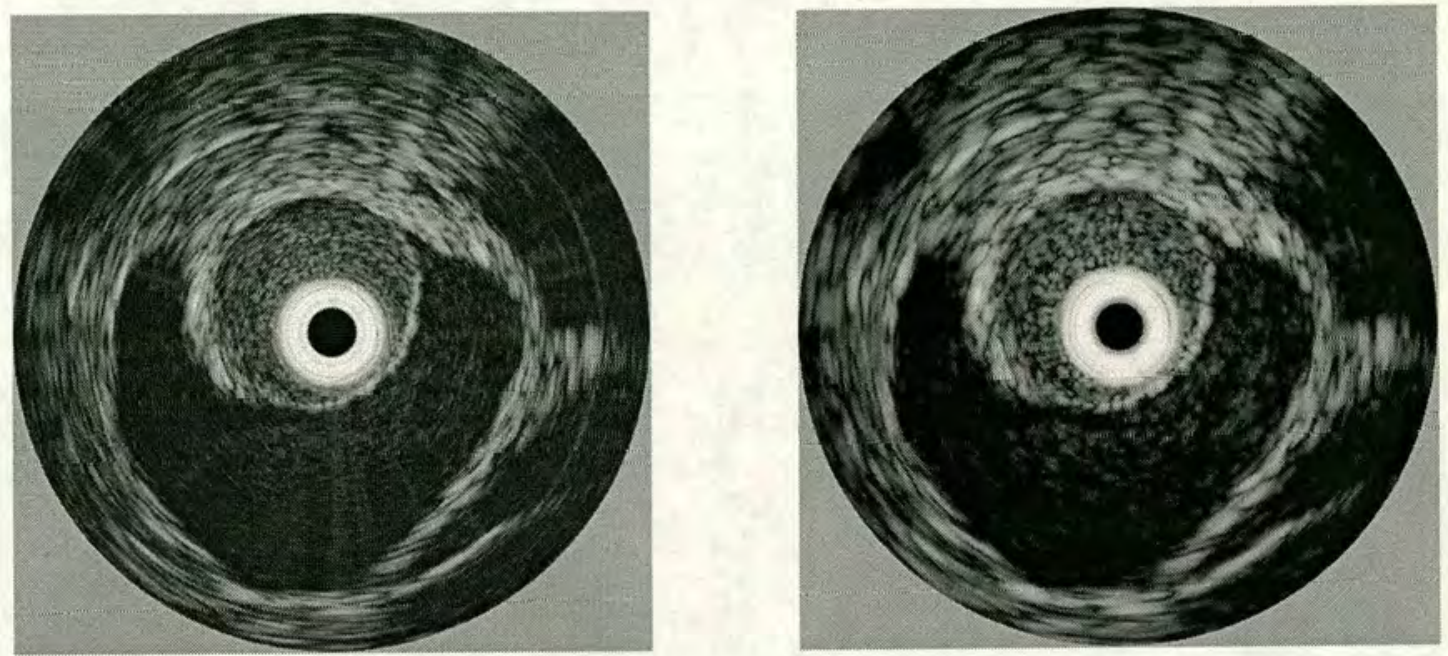

Figure 3.6 Example IVUS image (left) and after being filtered with a matched filter (right).

\subsection{Inverse filter}

An inverse filter is designed to completely reverse (or equalise) the effect of a channel (or any linear filter process) on a signal, and will work perfectly in the absence of noise, for stationary channels - giving an output identical to the initial system input in the case of Figure 3.1 and Figure 3.2.

The filter can be designed in the frequency domain by inverting the magnitude and negating the phase of every frequency component (usually achieved by performing a Fourier transform) as defined in equation 3.5. Once designed in the frequency domain, an inverse Fourier transform is required to convert the result back to the time domain. The result of the inverse Fourier transform will be the filter taps required to form an FIR, inverse filter. 
$\mathrm{X}(\omega)=\frac{\mathrm{H}^{*}(\omega)}{|\mathrm{H}(\omega)|^{2}}=\operatorname{Arg}\left\{\mathrm{H}^{*}(\omega)\right\} \cdot|\mathrm{H}(\omega)|^{-1}$

\subsubsection{Example design of an inverse filter}

This design process is shown below. Figure 3.7 shows a system impulse response and its corresponding inverse filter. The $y$-axis scales in Figure 3.7 are arbitrary. The design for this filter is performed in the frequency domain, as shown in Figure 3.8.
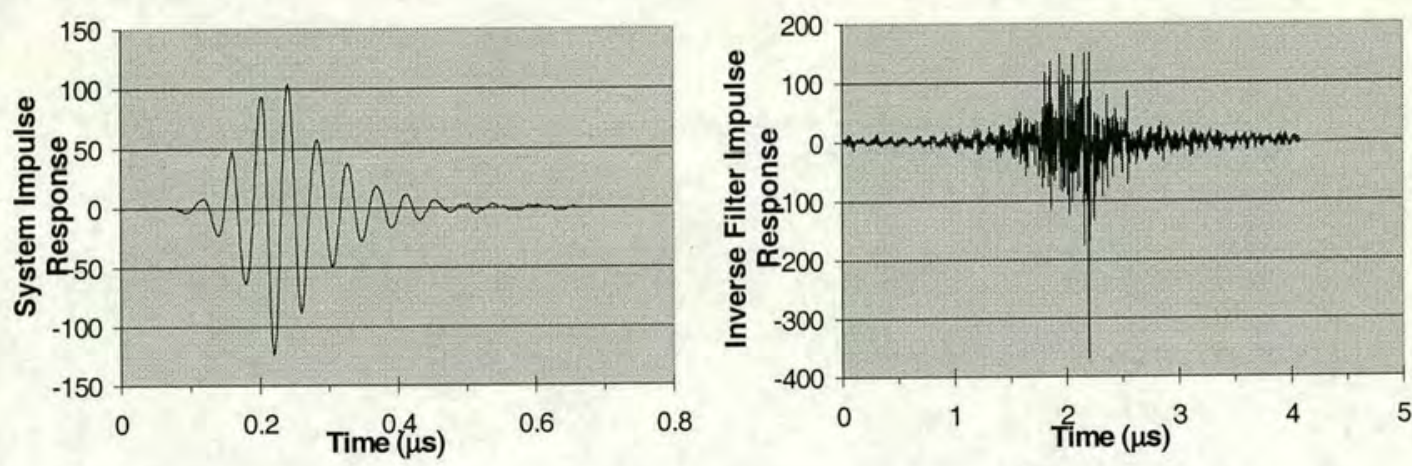

Figure 3.7 An inverse filter in the time domain (right) designed from the system impulse response (left).
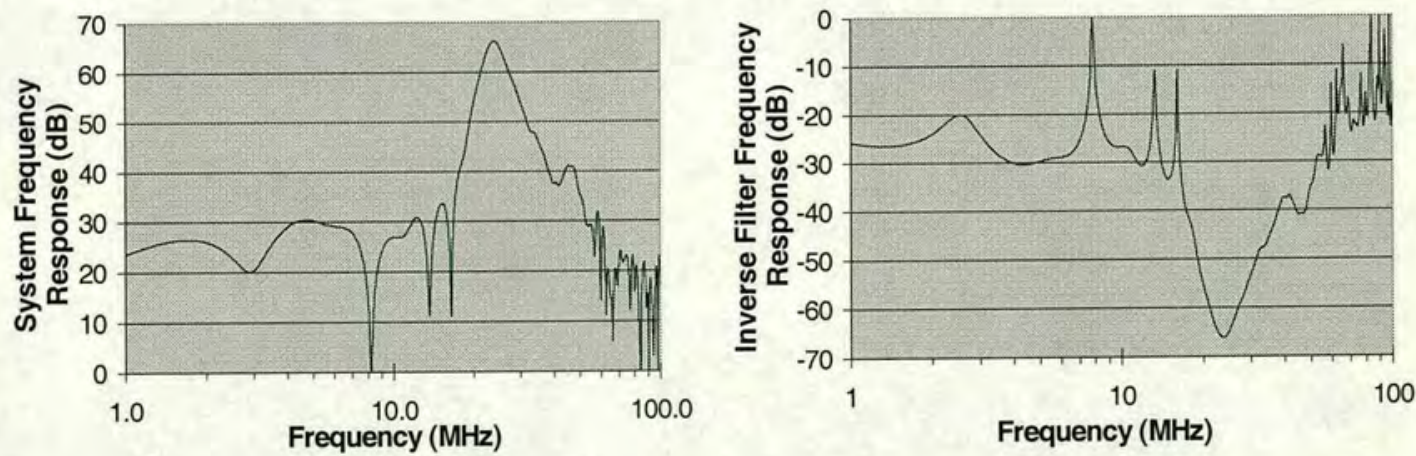

Figure 3.8 Magnitude response of an inverse filter (right) designed by inverting the magnitude of the system impulse response (left).

In the example design performed here, the inverse filter's impulse response is found to be concentrated within a window between 1 and $3 \mu$ s (see Figure 3.7, right). In 
order to construct the inverse filter as an FIR filter, it is required to truncate the filter tap series. This causes this filter to be not a true inverse filter, but only an approximation. The filter will be a close approximation if the tap sequence is long enough to include the majority of the "ideal" inverse filter impulse response. Therefore the sequence of taps in the inverse filter is required to be long enough to include (at least) the window between 1 and $3 \mu \mathrm{s}$ - that is, approximately three times the duration of the system's impulse response. In this instance the filter has been allowed to be approximately six times as long.

In practise an inverse filter will not work perfectly if there is noise entering the system. This is because the inverse filter amplifies Fourier components with low signal levels; the presence of noise at these frequencies will result in the filter amplifying the noise. Also, in any digital system there is always some quantisation noise. Hence a digital inverse filter will inherently never quite achieve a perfect inverse.

\subsubsection{Example application of an inverse filter}

Note that the example IVUS image in Figure 3.6 (left) has had a significant amount of noise amplified after application of an inverse filter - as shown in Figure 3.9.

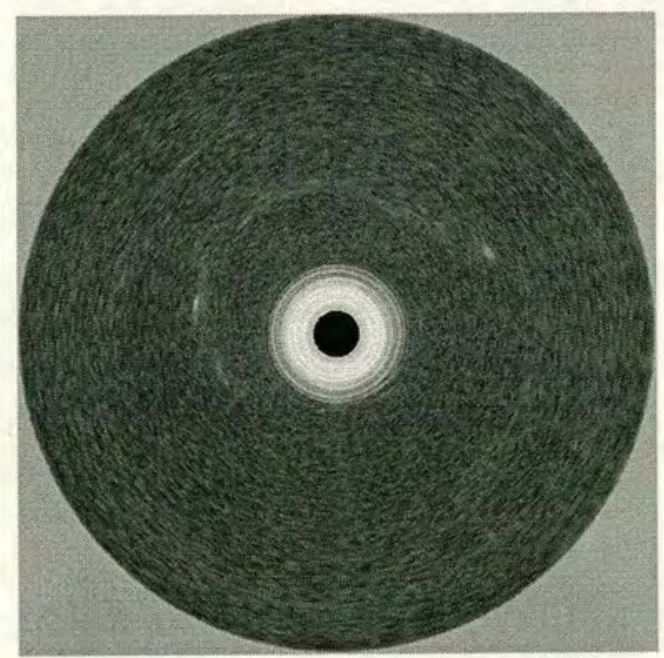

Figure 3.9 Example IVUS image after being filtered with an inverse filter. 


\subsection{Wiener filter}

A Wiener filter [97] is designed to minimise the mean square error between the filter output and the desired output, for any system containing noise such as in Figure 3.1 [98]. The minimum mean square error (MMSE) criteria is called the "cost function". The MMSE cost function is convenient and commonly chosen because its solution is well known and is always solvable. Although other cost functions can be used, they are not always tractable.

Wiener filters can be designed in either the time domain or the frequency domain. The equation for a MMSE Wiener filter is given in equation 3.6 in terms of the crosscorrelation vector (defined in equation 3.8) and the autocorrelation matrix (defined in equation 3.9). The resulting expected mean square error (MSE) from such a filter is given in equation 3.7 .

$$
\mathrm{x}_{\text {opt }}=\Phi_{h h}^{-1} \cdot \Phi_{h \delta}
$$

$$
\xi_{\text {opt }}=\left[\delta^{2}(n)\right]-\mathrm{x}_{\text {opt }}^{T} \Phi_{h \delta}
$$

$$
\Phi_{h \delta}=E[\mathrm{~h}(n) \delta(n)]
$$

$$
\Phi_{h h}=E\left[\mathrm{~h}(n) \mathrm{h}^{T}(n)\right]=E\left[\mathrm{~h}(n) \mathrm{h}^{T}(n)\right]_{\text {Free }}+E\left[\mathrm{~N}_{n}(n) \mathrm{N}_{n}^{T}(n)\right]
$$

For a MMSE filter, the Wiener filter is limited by the matched filter and the inverse filter. The Wiener filter approaches the matched filter when the signal to noise ratio is very low, and approaches the inverse filter when the signal to noise ratio is very high.

In order to design a Wiener MMSE filter, you need to know the autocorrelation of the system impulse response, and the system impulse response cross correlated with the 
desired output (or system input). Alternatively a Wiener MMSE filter can be designed in the frequency domain, in this case you need to know the power spectral density of the system impulse response, and the system's transfer function multiplied by the spectrum of the desired output signal.

The calculation of the autocorrelation matrix in equation 3.9 requires the designer to know or estimate the expected noise level $\mathrm{N}(n)$ in the observed sequence $\mathrm{y}(n)$. If the noise is uncorrelated with the signal $\mathrm{x}(n)$ then the noise will not affect the crosscorrelation vector in equation 3.8. However the noise will always affect the autocorrelation matrix. For uncorrelated noise, the expected value of $\mathrm{N}(n) \mathrm{N}^{\mathrm{T}}(n)$ is zero everywhere except on the leading diagonal of the autocorrelation matrix, where the value is equal to the total power of the noise. In order to be safe, and achieve a better filter performance, Wiener filters are often designed for a noise level slightly higher than what is measured or expected $[99,100]$.

\subsubsection{Example design of a Wiener filter}

Figure 3.10 shows a Wiener MMSE filter designed in the time domain using equation 3.6. In designing this example Wiener MMSE filter, the following assumptions were made:

- the observed system impulse response was a good estimate of the noise free system impulse response;

- the system input was a dirac delta function. (i.e. a perfect impulse);

- the desired output was also a dirac delta function;

- the noise is uncorrelated with the signal and with itself;

- the noise is AWGN.

These assumptions are reasonable in many situations, but will not always be valid. e.g. in ultrasound imaging systems, the noise seems to be highly correlated with the 
signal, and multiplicative rather than additive. This means that the Wiener filter (when designed using the assumptions above) may not be optimal for ultrasound imaging applications.

If the desired output or system input is not a dirac delta function - but is known then the Wiener filter can still be calculated and will be tractable. However, if the noise in the system is correlated then the equations to calculate a Wiener filter may not be solvable.

For this example, it was assumed that the noise power was $1 \%$ of the strength of the signal power $(\mathrm{SNR}=20 \mathrm{~dB})$. In general it is desirable to estimate or measure the noise power level. For the purposes of an example design however, it is sufficient to assume the noise level.

Figure 3.10 shows the system impulse response, and the example Wiener filter response in the time domain. Figure 3.11 shows these in the frequency domain.
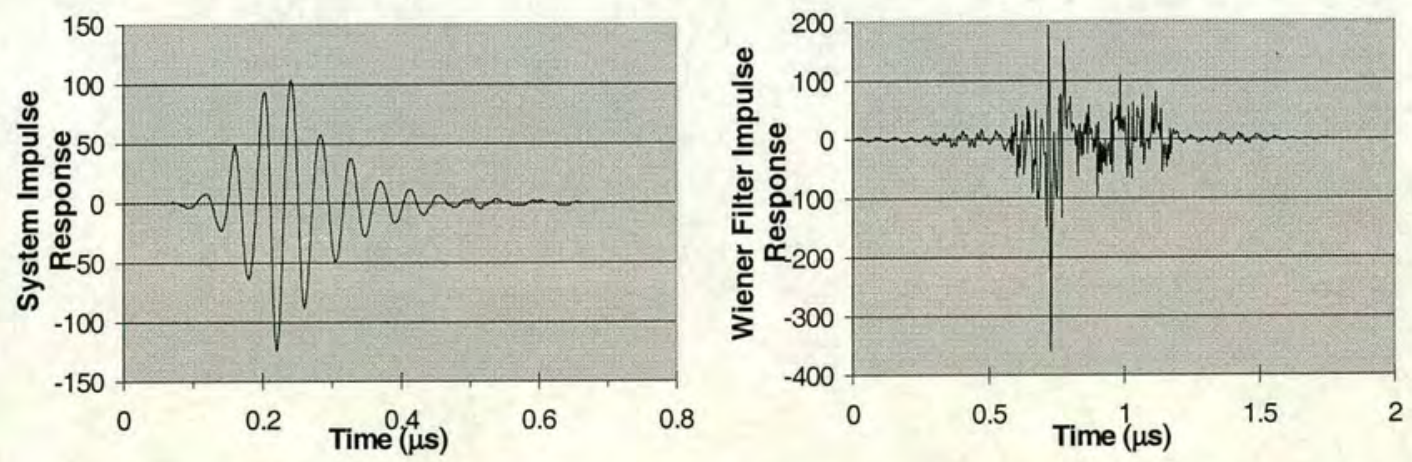

Figure 3.10 A Wiener MMSE filter in the time domain (right) assuming SNR $=20 \mathrm{~dB}$ for the system impulse response (left).

It can be clearly seen from Figure 3.11 that the response of the Wiener filter is somewhat like an inverse filter (in the passband region), and is somewhat like a matched filter (outside the passband region). The width of the passband region referred to here is not well defined; it is dependant on the level of noise specified in the filter design (in this case $1 \%$ or $\mathrm{SNR}=20 \mathrm{~dB}$ ). 

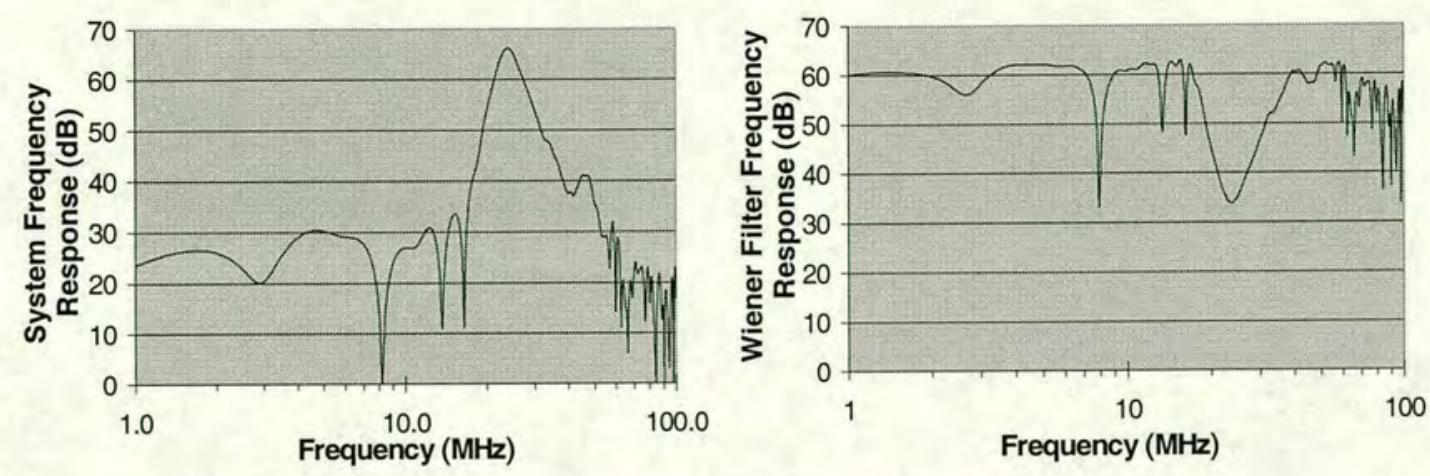

Figure 3.11 The magnitude response of a Wiener MMSE filter (right) and the magnitude of the system impulse response (left)

The Wiener filter has treated the downward notches in the system's impulse response very differently from the inverse filter. The inverse filter amplifies any noise or signal present at these frequencies, but the Wiener filter attenuates it. This makes the Wiener filter perform better than the inverse filter in the presence of noise.

\subsection{Phase correction filter}

A phase correction filter is designed such that the magnitude of any frequency component will remain unchanged after passing through the filter. However the phases of each frequency component will change. The filter will typically be designed to cancel (or conjugate) the phase shift(s) of the system impulse response.

This filter is not widely used, but is useful as a reference. After filtering with this filter the only effects remaining from the distorting channel will be the changes to the magnitude of each Fourier component.

\subsubsection{Back propagation}

Phase correction filters come in several types; a technique called "back propagation" has been shown to be a very useful technique for correcting phase aberrations. 


\section{The backpropagation algorithm}

The model used for implementing back propagation phase correction by Liu and Waag [101] uses a phase screen, and is applied to transmission ultrasound only. The actual imaging situation is shown in Figure 3.12. Figure 3.13 shows the model that is assumed when applying the back propagation algorithm.

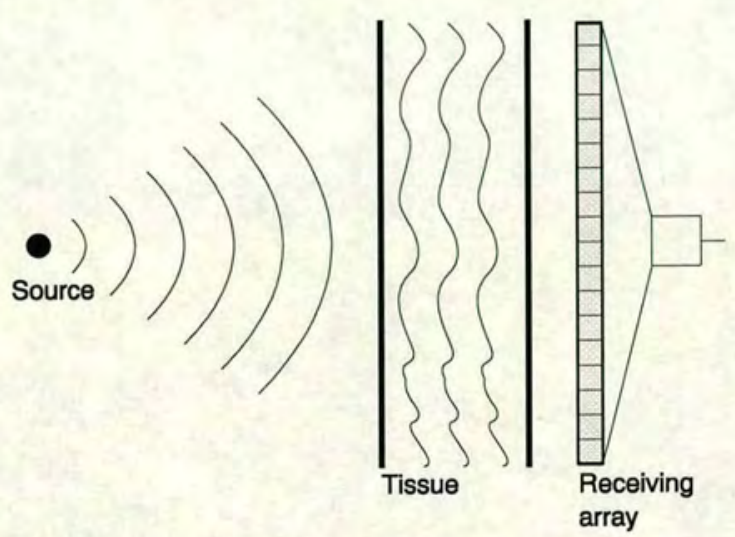

Figure 3.12 Transmission based ultrasound imaging system with an array of receiving elements.

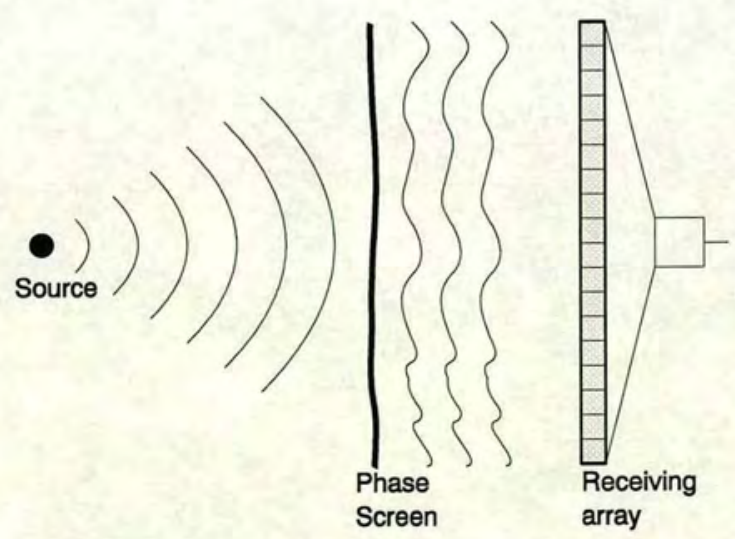

Figure 3.13 The model assumed in applying backpropagation.

In order to apply backpropagation it is essential to have an array of receiving elements so that the received wavefront can be Fourier transformed into an angular spectrum of plane waves. Each plane wave is then backpropagated by a given distance (the distance to the assumed phase screen), and inverse transformed to 
reconstruct the waveform at that point. The waveform is calculated at every point across the phase screen - that is, at as many points as there are receiving elements.

Backpropagated waveforms are calculated for many different distances, (or possible phase screen positions).

At every position on the phase screen, and at every distance, a time shift is applied to the (back propagated) signal, in such as way as to achieve a constant arrival time across the phase screen (allowances for geometric differences may be made). Finally, each different reconstructed waveform (one for each different possible phase screen position) is compared with a reference waveform to see which one is the best available match to the waveform that was expected from the source. In order to provide the reference waveform, the waveform expected from the source must be estimated or measured.

This method has been shown by Liu and Waag [101] to produce results that are superior to the more simple method of applying a time shift to every signal at the receiving array. It has been found by Zhu and Steinberg [56] that this technique also produces results that are superior to phase correction at the receiving elements.

Since the time shifting is done element by element, this effectively makes backpropagation a type of phase manipulation or phase correction. The major difference is that in backpropagation the phase of each Fourier component at each receiving element is being adjusted in a manner that is affected by the signal received at other elements.

\section{Back propagation and IVUS}

Phase correction by back propagation is applied to transmission ultrasound only. The technique as presented will not work with reflective ultrasound due to interference effects obscuring the details of the phase shift. The interference will prohibit estimation of the arrival time of single pulses, so it is not possible to time shift the signal at each element to get a constant arrival time. Because back propagation is 
utilised in transmission ultrasound only (as opposed to reflective ultrasound), this makes its application to conventional IVUS imaging impossible since all IVUS imaging necessarily uses reflective ultrasound.

Another feature of the back propagation algorithm is that it relies on having an array of receiving elements. The algorithm cannot be applied to single element transducer systems where there is only one receiving element. Since most IVUS imaging systems use single element transducers (in order to make the transducer physically small), this is a second reason why back propagation cannot be used with IVUS imaging systems.

\subsubsection{A simple phase correction filter}

A simple phase correction filter can cancel the phase changing effects of a distorting channel, while leaving the amplitude unchanged. In practise, for a digital filter, the amplitude response at all frequencies cannot be precisely unity, but a good approximation to this can be made.

The filter is calculated in the frequency domain by negating the phase of every Fourier component, and setting the magnitude of every Fourier component to a constant (positive and non-zero) value. The filter taps are formed by inverse transforming to the time domain. The design equation is shown in equation 3.10:

$\mathrm{X}(\omega)=\frac{\mathrm{H}^{*}(\omega)}{|\mathrm{H}(\omega)|}=\operatorname{Arg}\left\{\mathrm{H}^{*}(\omega)\right\} \cdot 1$

\subsubsection{Example design of a simple phase correction filter}

The example waveform and filter shown in Figure 3.14 was calculated using a fast Fourier transform (FFT) that was much longer than the duration of the impulse response. This yields greater accuracy, but makes the filter long. The filter shown in 
Figure 3.14 has been truncated to show the main region of interest (taps outside this region are close to zero).
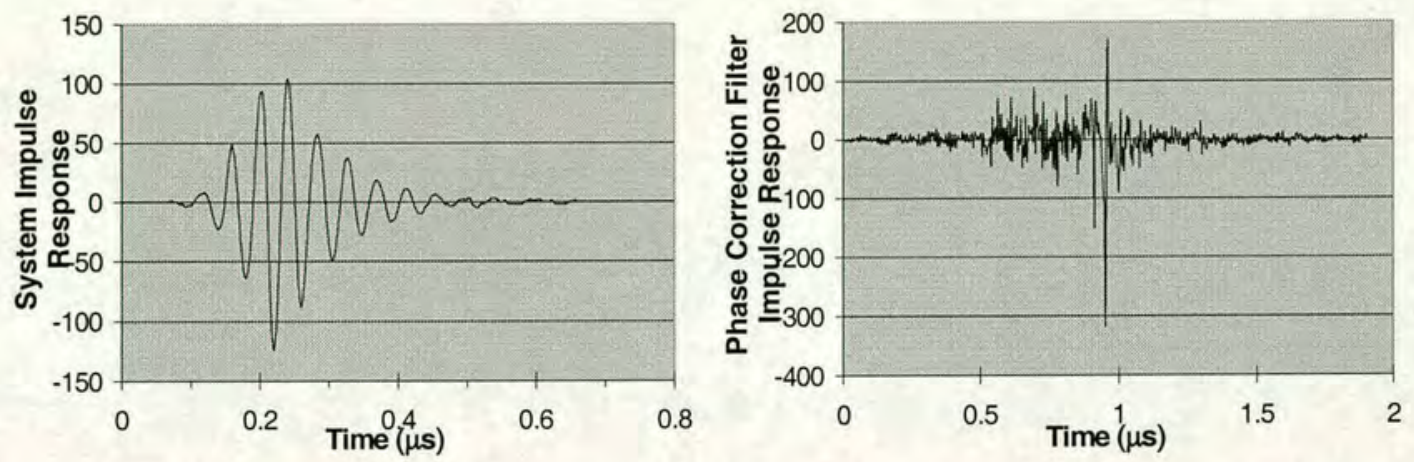

Figure 3.14 A phase correction filter in the time domain (right) designed from the system impulse response (left).
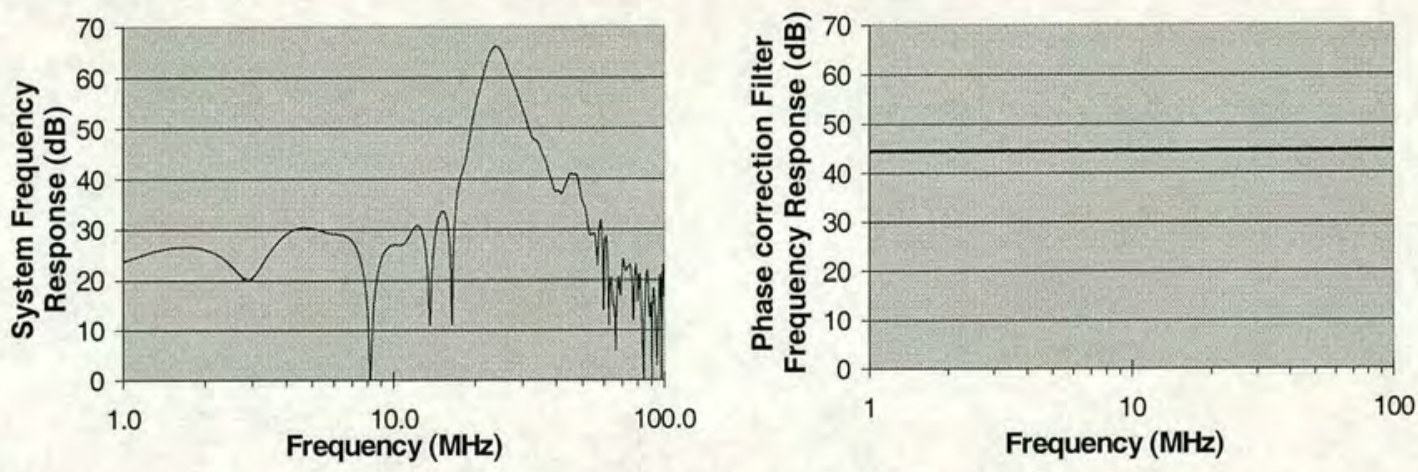

Figure 3.15 The magnitude response of a phase correction filter (right) designed from the system impulse response (left)

Truncating the series of filter taps will alter the filter's frequency response. The truncation can be done either by simply truncating the tap sequence, or by applying a window function to the tap sequence. (The simple case equates to using a rectangular window function). The effects of this are shown in Figure 3.16 for a rectangular and a Hann window. In both cases the length of the filter has been reduced by a factor of approximately five, but is still more than three times as long as the measured impulse response. The frequency response has changed, and is no longer ideal. Although the frequency response curve is smoothed in the case of the Hann window, it is just as far from ideal as the rectangular window case. 

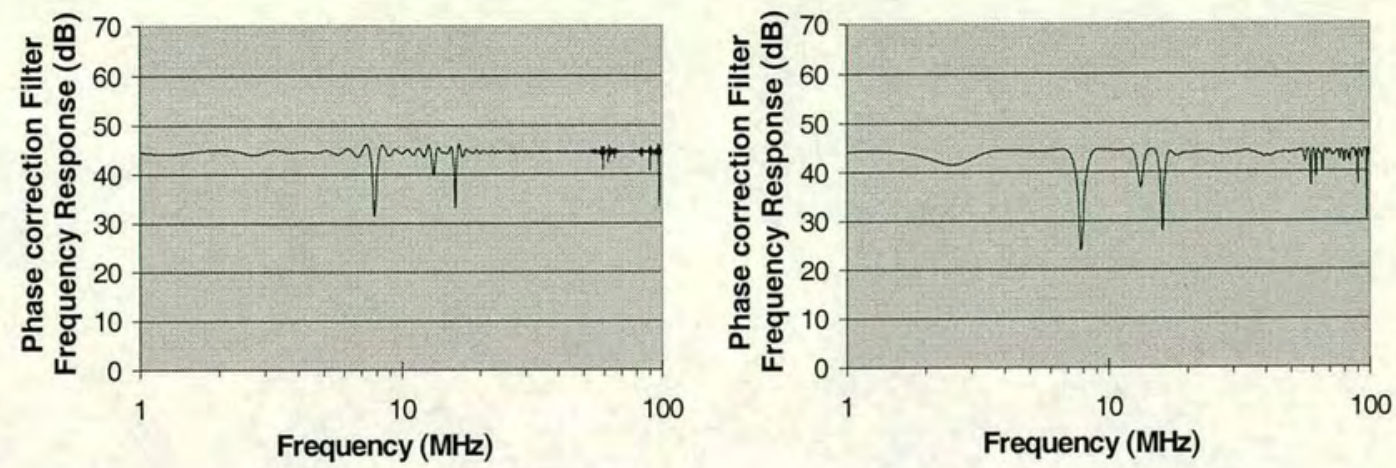

Figure 3.16 Magnitude response of a phase correction filter response when the filter series is truncated with a rectangular window (left) and a Hann window (right).

\subsection{Square root filter}

This filter has been proposed for application to medical ultrasound by Zhu and Steinberg [56]. They propose that the filter will be "towards inverse filtering", by providing amplitude compression i.e. making the magnitudes of all Fourier components closer to unity (after normalisation). They applied this filter to transmission based ultrasound, and they report very favourable results.

The square root filter is designed to be a compromise that is half way between a phase correction filter and an inverse filter - providing spectral flattening, while not excessively amplifying noise. When designing the filter, the magnitude of each Fourier component is inverse square rooted (or raised to the power $-1 / 2$ ) and the phase is conjugated - see equation 3.11 :

$\mathrm{X}(\omega)=\frac{\mathrm{H}^{*}(\omega)}{|\mathrm{H}(\omega)|^{1.5}}=\operatorname{Arg}\left\{\mathrm{H}^{*}(\omega)\right\} \cdot|\mathrm{H}(\omega)|^{-1 / 2}$ 


\subsubsection{Example design of a square root filter}

The example waveform shown in Figure 3.17 (left) was Fourier transformed, inverse square rooted and phase conjugated as in equation 3.11. The Fourier transform was calculated using an FFT that was much longer than the duration of the impulse response (as with the phase correction filter). This yields greater accuracy, but makes the filter long. The filter shown in Figure 3.17 has been truncated to show the main region of interest.
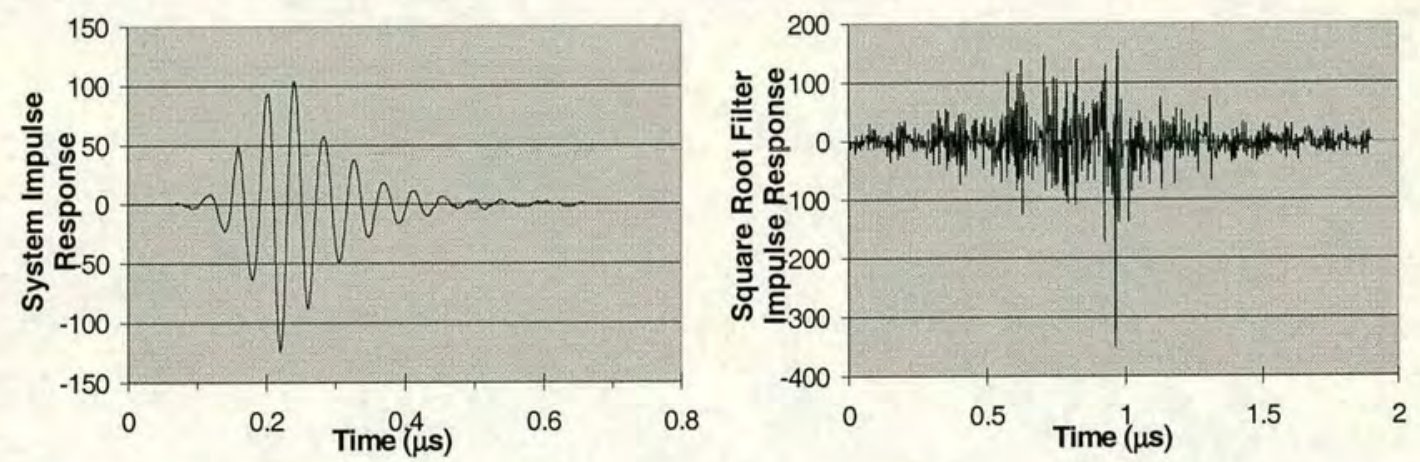

Figure 3.17 A square root filter in the time domain (right) designed from the system impulse response (left).
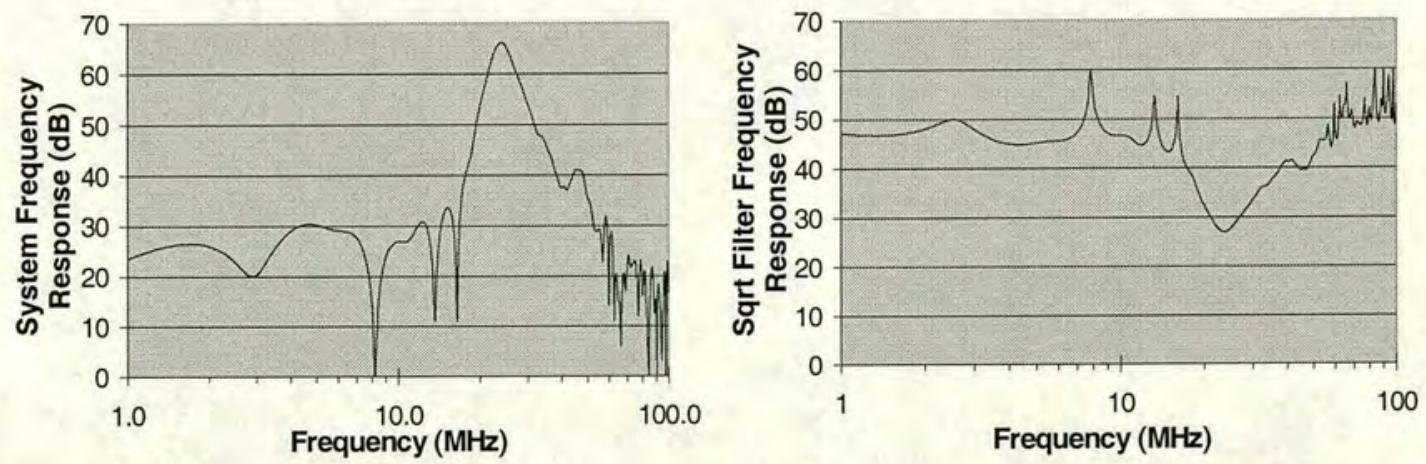

Figure 3.18 The magnitude response of a square root filter (right) designed from the magnitude of the system impulse response (left)

Figure 3.18 shows that the square root filter approaches an inverse filter, but any smaller Fourier components are not fully inverted, they are raised only to the power of $-1 / 2$ instead of being raised to the power -1 as the inverse filter does. This makes 
the filter less sensitive to noise at these frequencies, and provides the motivation for this filter.

Truncating the series of filter taps will alter the filter's frequency response in a similar manner to the effect of truncating the series of taps of the phase correction filter. The number of filter taps could also be shortened by designing the filter with a shorter FFT.

\subsubsection{Fourth root filter}

Zhu and Steinberg [56] extended the concept of a square root based filter to a filter where the magnitude of the Fourier components are raised to any arbitrary power. Thus, the filter can be designed arbitrarily close to (or removed from) the design of an inverse filter which has a power of -1 .

The equation for a filter with Fourier components raised to any arbitrary power is given in equation 3.12 , where $n$ can take on any chosen (non-zero) value. This yields an $n^{\text {th }}$ root filter; the value $n$ is not necessarily restricted to positive integers.

$$
\mathrm{X}(\omega)=\frac{\mathrm{H}^{*}(\omega)}{|\mathrm{H}(\omega)|^{2-1 / n}}=\operatorname{Arg}\left\{\mathrm{H}^{*}(\omega)\right\} \cdot|\mathrm{H}(\omega)|^{-(n-1 / n)}
$$

In particular, Zhu and Steinberg [56] proposed a fourth root filter (setting $n=4$ ), which results in the filter design in equation 3.13 :

$$
\mathrm{X}(\omega)=\frac{\mathrm{H}^{*}(\omega)}{|\mathrm{H}(\omega)|^{1.75}}=\operatorname{Arg}\left\{\mathrm{H}^{*}(\omega)\right\} \cdot|\mathrm{H}(\omega)|^{-3 / 4}
$$

The fourth root filter is closer to an inverse filter than is the case in the square root filter. Zhu and Steinberg [56] reported favourable results from the fourth root filter under some (but not all) conditions. 


\subsubsection{The matched, phase correction, and inverse filters as $n^{\text {th }}$ root filters}

The square root and fourth root based filters use phase correction as found in the matched filter, the phase correction filter and the inverse filter.

The magnitude of the Fourier components of the matched filter, the phase correction filter, the square root filter, the fourth root filter, and the inverse filter can all be described by appropriate values of $n$ in equation 3.12. Table 3.1 shows the values of $n$ required for each filter, the magnitude of the filter response, and the magnitude of a filtered system impulse response.

\begin{tabular}{|l|l|l|l|}
\hline Filter type & $\begin{array}{l}\text { Value } \\
\text { of } n\end{array}$ & $\begin{array}{l}\text { Magnitude of filter } \\
\text { response } \mathrm{X}(\omega)\end{array}$ & $\begin{array}{l}\text { Magnitude of filtered system } \\
\text { impulse response } \mathrm{Y}(\omega)\end{array}$ \\
\hline Matched filter & $1 / 2$ & $\mathrm{X}(\omega)=\mathrm{H}(\omega)^{1}$ & $\mathrm{Y}(\omega)=\mathrm{H}(\omega)^{2}$ \\
\hline Phase correction filter & 1 & $\mathrm{X}(\omega)=\mathrm{H}(\omega)^{0}=1$ & $\mathrm{Y}(\omega)=\mathrm{H}(\omega)^{1}$ \\
\hline Square root filter & 2 & $\mathrm{X}(\omega)=\mathrm{H}(\omega)^{-1 / 2}$ & $\mathrm{Y}(\omega)=\mathrm{H}(\omega)^{1 / 2}$ \\
\hline Fourth root filter & 4 & $\mathrm{X}(\omega)=\mathrm{H}(\omega)^{-3 / 4}$ & $\mathrm{Y}(\omega)=\mathrm{H}(\omega)^{1 / 4}$ \\
\hline Inverse filter & $\infty$ & $\mathrm{X}(\omega)=\mathrm{H}(\omega)^{-1}$ & $\mathrm{Y}(\omega)=\mathrm{H}(\omega)^{0}=1$ \\
\hline
\end{tabular}

Table 3.1 Exponents of Fourier components for various filters.

By the appropriate use of $n$, equation 3.12 can form filters that can range from the inverse filter, through to (and beyond) a matched filter. Therefore it is fair to say that the square root (and fourth root) filter is a trade-off between the inverse and matched filter. 


\subsection{Other attempts at pseudo inverse filtering of ultrasound}

\subsubsection{Modified Wiener filters}

Yamada and Yamabe have presented a technique which is a type of pseudo inverse filter [55]. They fail to specify fully their filter design, but it appears to be based around a Wiener filter with an assumption that the desired output of the filter (for a system impulse response plus noise as input) is not a perfect impulse (dirac delta function), but a band limited impulse, with hard cut-offs. This assumes that the system passes a certain bandwidth perfectly, and frequencies outside the bandwidth are perfectly attenuated. Observations of ultrasonic systems impulse responses indicate that this assumption is not valid, and therefore this will lead to a non-optimal filter. However it is acceptable as a first order approximation - this is verified in that favourable results were reported.

Other researchers have also developed modified Wiener filters, although not for use with medical ultrasound images [102, 103, 104, 105, 106, 107].

\subsubsection{SVD based filter}

Shen and Ebbini $[57,58]$ have proposed a filter for ultrasound applications that is based upon a Singular Value Decomposition (SVD) [98]. It is intended for use with their proposed $3 \mathrm{D}$ coded excitation system $[59,60,61]$, whereby the transmitted ultrasound signals will be deliberately spread in time using coded pulses to a phased array transducer. The design approach uses rank reduction to obtain an approximation to the inverse filter.

The filter presented did provide some degree of inversion with part of the system passband. However not all of the passband frequencies were inverted in the reported results. 
The approach given by Shen and Ebbini is applied strictly to a phased array system, and will not work for a single element transducer system. This filter is not considered here because of its inability to work with single element IVUS systems.

\subsection{A comparison of the above filters}

In order to compare the filters presented in this chapter, the model of Figure 3.3 will be used. In this model, the filter $\mathrm{x}(n)$ or $\mathrm{X}(\omega))$ takes in $\mathrm{h}(n)$ or $\mathrm{H}(\omega)$, and gives out $\mathrm{y}(n)$ or $\mathrm{Y}(\omega)$. The magnitude responses of $\mathrm{H}(\omega), \mathrm{X}(\omega)$ and $\mathrm{Y}(\omega)$ will be compared. This will enable a comparison of how each filter treats different magnitudes of Fourier components.

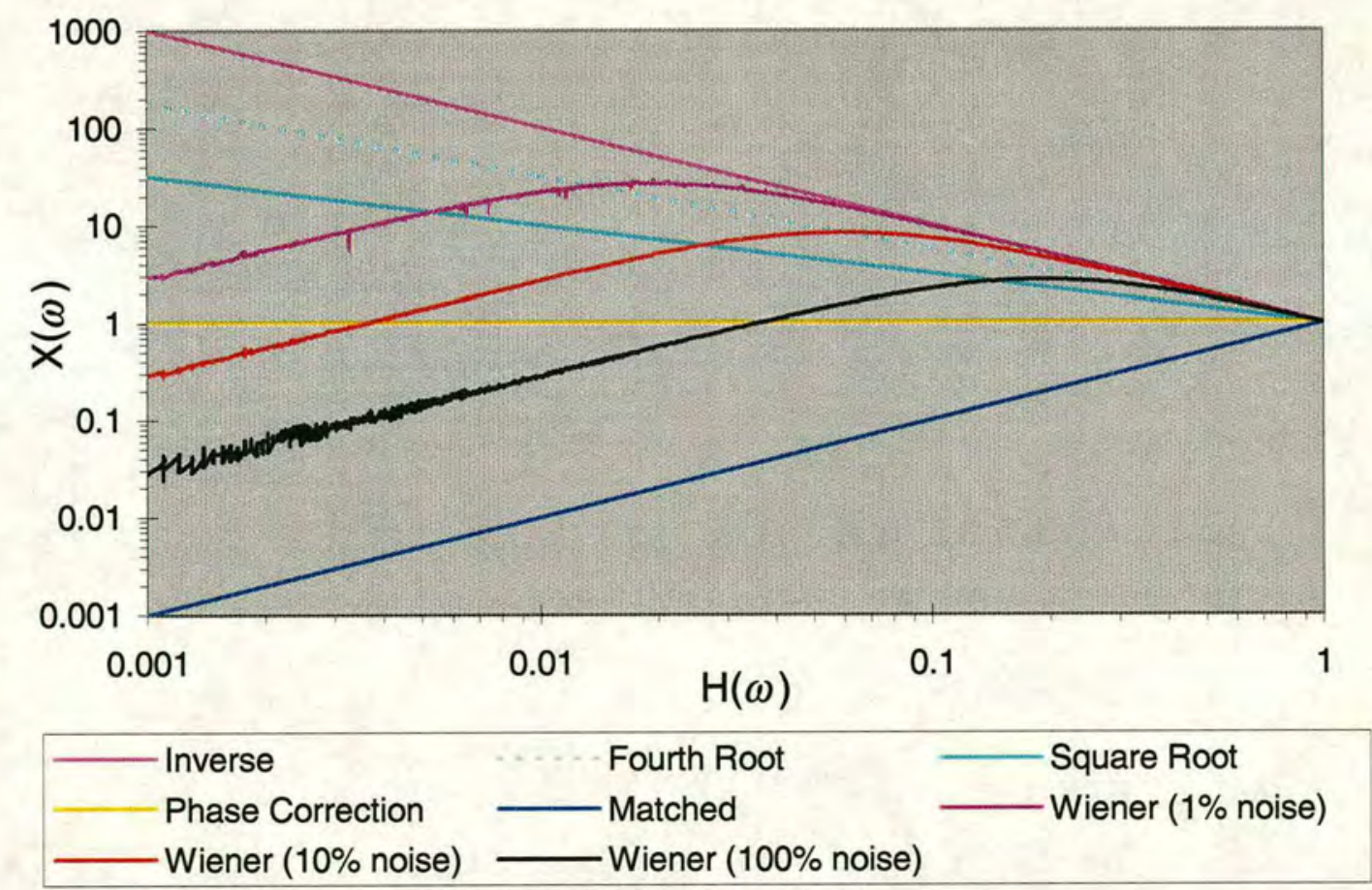

Figure 3.19 Filter Transfer function - designed for the same signal $h(n)$.

For Figure 3.19 and Figure 3.20, $\mathrm{H}(\omega)$ has been normalised. All the filter response curves have been normalised so that the largest component of $\mathrm{H}(\omega)$ gives a filter output of unity. 


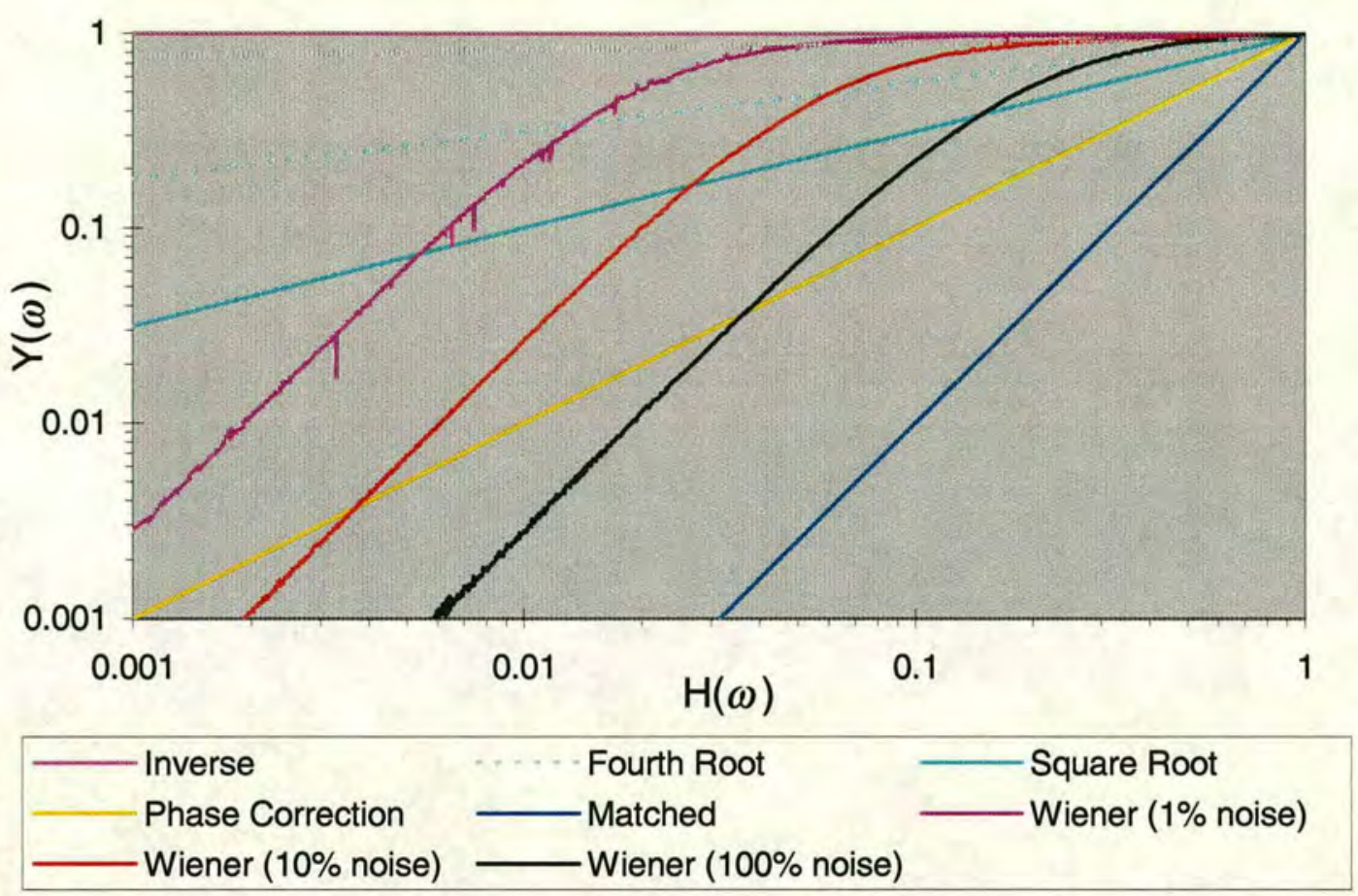

Figure 3.20 Filter responses - designed for the same signal $h(n)$.

Both Figure 3.19 and Figure 3.20 show that where the $H(\omega)$ Fourier components are large, the Wiener filter response is close to an inverse filter; closer than either the square root or fourth root filters. However, as the $H(\omega)$ Fourier components get smaller, the Wiener filter attenuates the signals more than both the fourth root filter, the square root filter, and even the phase correction filter.

The three Wiener MMSE filter curves shown each correspond to a different assumed level of noise being added to the signal in the model of Figure 3.1. The three curves correspond to assumed noise power levels of $1 \%, 10 \%$, and $100 \%$ of the signal power. The Wiener filters with lower assumed noise power levels have a magnitude response that is closer to the inverse filter than the filters with higher assumed noise power levels. If the actual noise power level in the system is different from the assumed level, then the mean square error in the filter output will not minimised.

It is worth noting that in regions of small $\mathrm{H}(\omega)$ Fourier components, the Wiener filters begin to perform the same as a level shifted matched filter. 


\subsection{Conclusion}

There are many different possible methods for designing filters. Each method has its own particular merits, and is useful under some circumstances.

Most of the filters presented here are utilised as comparisons in later chapters. The results of applying these filters to images of a near perfect reflector can be found in Chapter 6. The results of applying these filters to IVUS medical images can be found in Chapter 8.

All of the filters described in this chapter (except for those in section 3.7) are implemented in the software on the accompanying CD. Instructions for the operation of the program that implements the design and application of these filters is described in Appendix C. 


\section{Chapter 4.}

\section{SIGMOID BASED PSEUDO}

INVERSE FILTER DESIGN

\subsection{Introduction}

This chapter describes a sigmoid based pseudo-inverse filter which is tailored to the characteristics of the ultrasound transducer (including phased array beamforming characteristics). This filter is designed to be tolerant of noise, while still performing a pseudo frequency inversion to obtain an approximation to a classical inverse (or deconvolution or spiking) filter. This filter is a modified version of a filter that was first presented [80, 108, 109] and applied in the analysis of foetal heart phonocardiogram (PCG) signals. The original presentation of this filter was in $\mathrm{H}$. Basil's Ph.D. thesis [80]. The relevant section of this thesis is reproduced in Appendix I. The application of this filter to ultrasound images has been recently published by myself [110] - this paper is reproduced in full in Appendix H.

\subsection{Background model assumptions}

It is assumed that the ultrasound transducer is initially excited with an impulse, that would ideally be a dirac delta function of infinitely short duration. However it will in practise be excited by a band-pass filtered dirac delta function as the voltage supply circuits will filter the impulse. The transducer then responds to the excitation, by emitting an ultrasound pulse. The pulse emitted can be considered as a shaped or filtered version of the voltage that excited the transducer. The transmitted pulse then propagates through the imaged medium, is reflected and some of the pulse returns to the transducer. This may also apply further filtering to the impulse. Finally the transducer receives the ultrasound reflections, converts them to a voltage and sends 
them to a digitiser. This receive process also shapes or filters the pulse. The convolution of all these filtering stages can be grouped together to form the system impulse response. This generalised system is shown for the case of imaging a perfect reflector in Figure 4.1 and is simplified by the model shown in Figure 4.2.

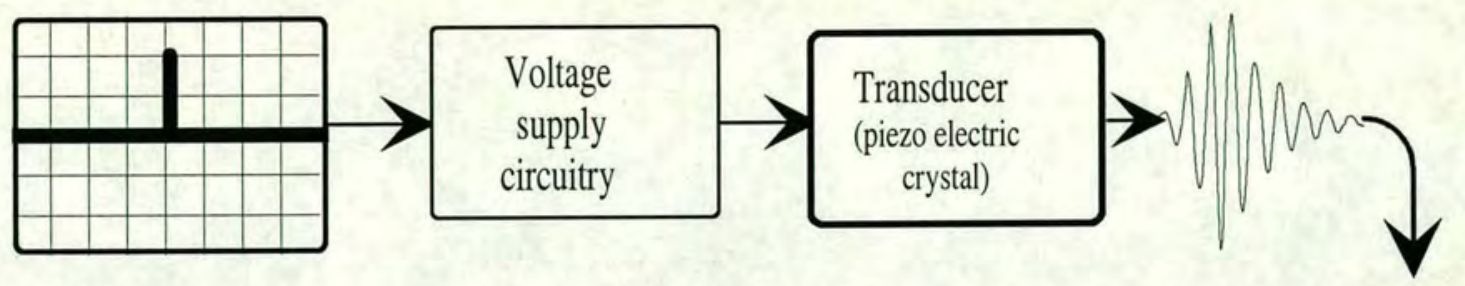

Reflected by a perfect reflector

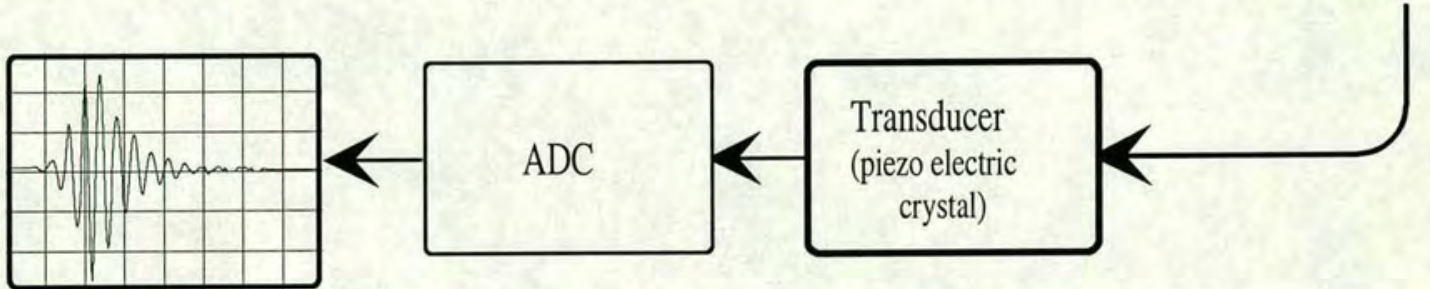

Figure 4.1 An ultrasound imaging system (with a perfect reflector)

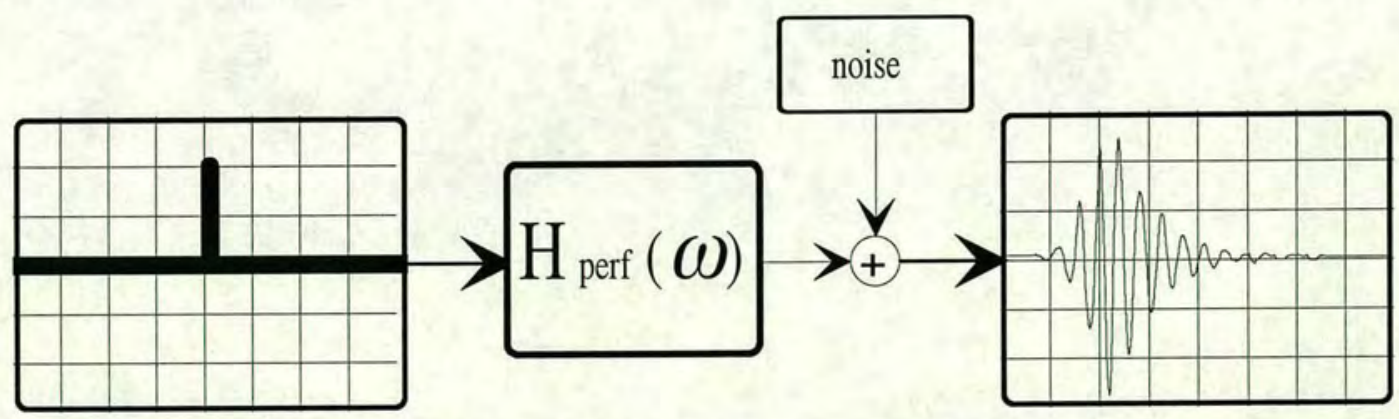

Figure 4.2 A model of an ultrasound imaging system (as in Figure 4.1) with a perfect reflector

Figure 4.2 is an accurate model only for the case of a perfect reflector as the target object. In this case the perfect reflector does not have any effect on the system impulse response $\mathrm{H}_{\text {perf }}(\omega)$. 
To allow for generalised target objects, which do affect the signal that returns to the receiver, the modified model as shown in Figure 4.3 is used. This introduces the target object with a response of $\mathbf{H}_{\text {image }}(\omega)$. $\mathbf{H}_{\text {image }}(\omega)$ will vary with position. In many ultrasound systems, the signal that returns to the receiver is used as an estimate of $\mathbf{H}_{\text {image }}(\omega)$ - after demodulation.

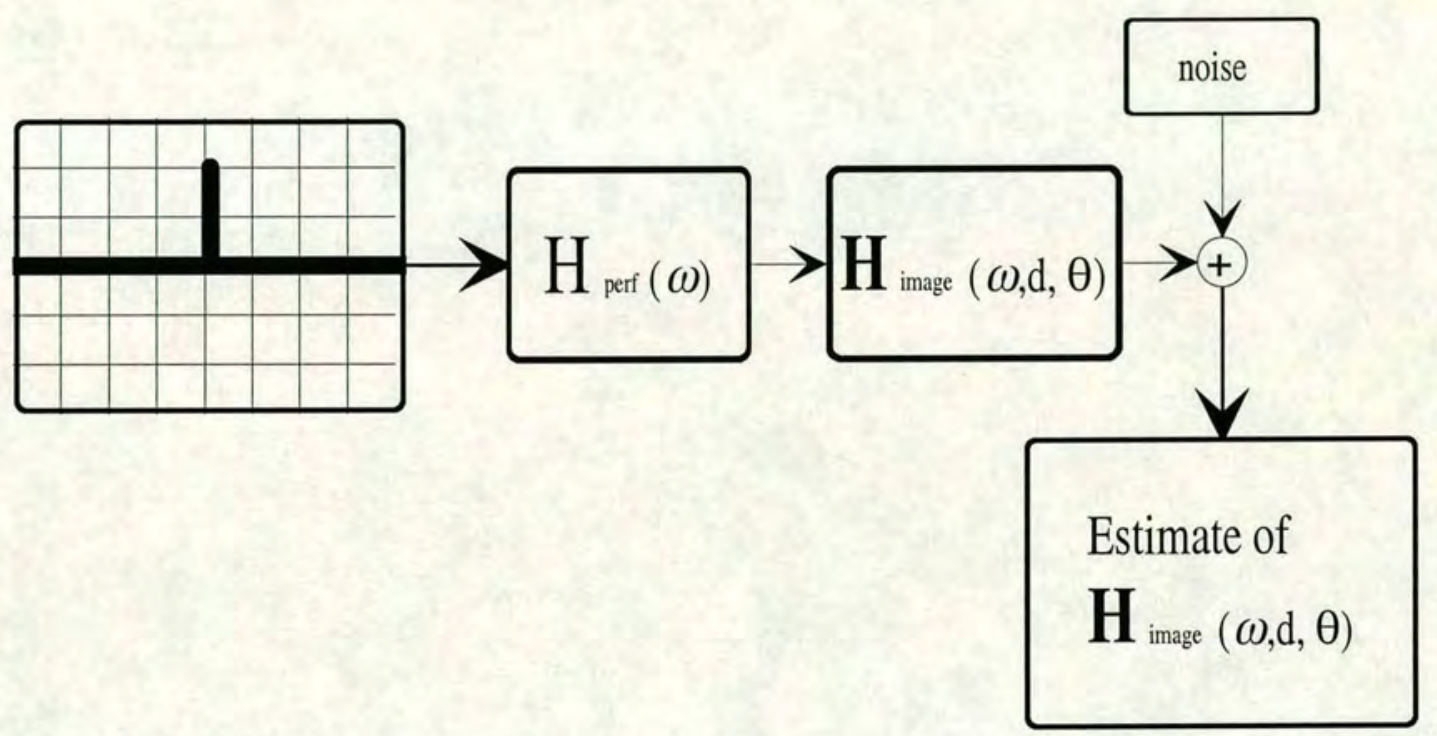

Figure 4.3 A model of an ultrasound imaging system with a target object

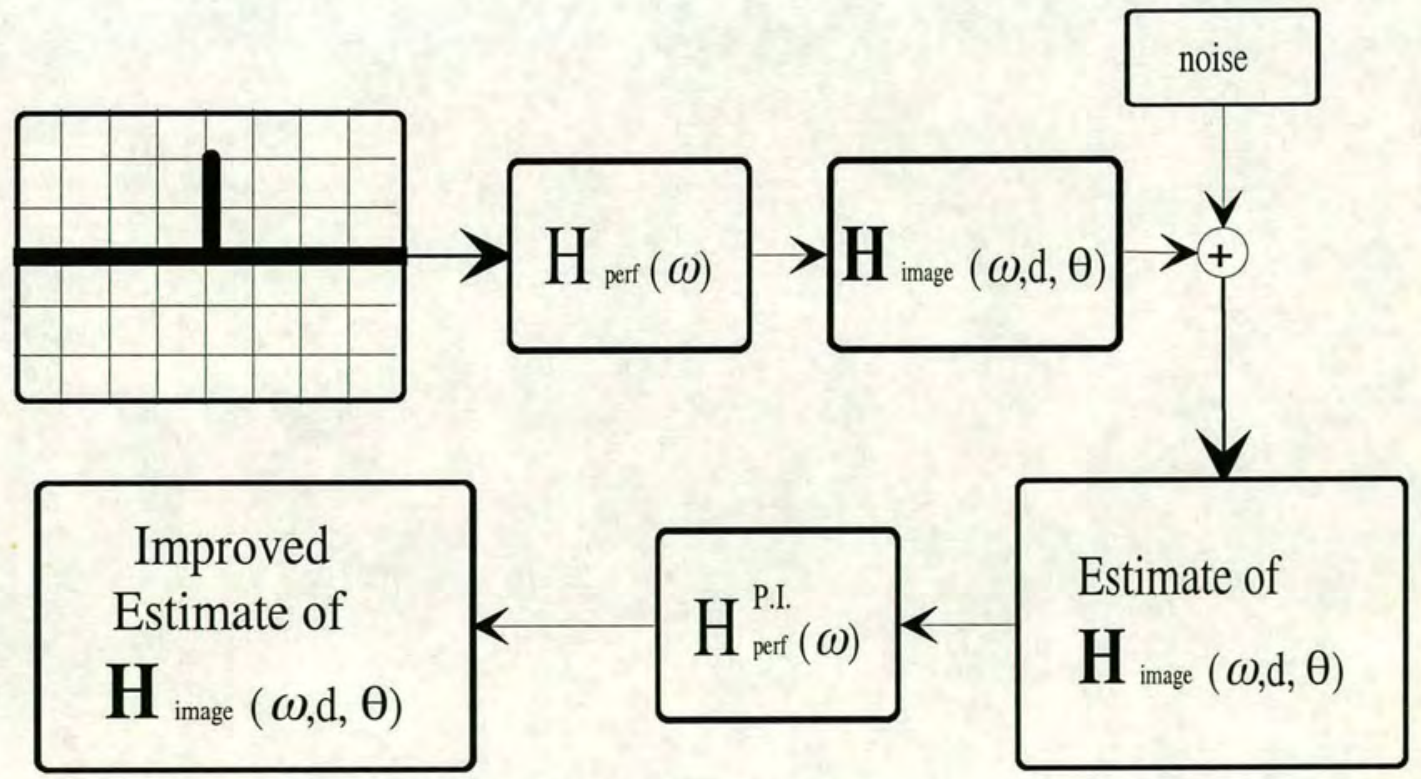

Figure 4.4 A model of an ultrasound imaging system with a target object with a pseudo inverse filter added to the system 
It is proposed to extend the model shown in Figure 4.3 with a pseudo inverse filter, as shown in Figure 4.4. The filter will be designed to partially negate the effects of the $\mathrm{H}_{\text {perf }}(\omega)$, which will result in an improved estimate of the $\mathbf{H}_{\text {image }}(\omega)$.

\subsubsection{Measuring the transducer's impulse response}

The ultrasound reflection from a near perfect plane reflector was measured using the system shown in Figure 4.1 (an example of the observed pulse is shown in Figure 4.5). This may be considered to be the transducer's impulse response (at one particular distance of the reflector). The near perfect plane reflector is made of glass or steel, and reflects the vast majority of the incident ultrasound. Its width is significantly greater than the beam width, and it is flat to within much less than one wavelength. This impulse response is then utilised in the filter design process. This allows a near perfect characterisation of the system impulse response (amplitude and phase).

Some researchers use a wire as a near perfect reflector, whereas a plane reflector was used here. Medical ultrasound imaging is usually concerned with determining 2D surfaces that are not perfectly flat, therefore both the plane reflector and the wire reflector do not fully represent the signals that will occur in practise. However both can offer a sufficiently accurate approximation to allow the construction of a filter to improve the image resolution.

An assumption was made that prior to the onset of the impulse response (at approximately $0.08 \mu$ s in Figure 4.5 ), there should be zero signal, and any received signal should be removed (assumed to be noise or very distant echoes from previously transmitted pulses). It was also assumed that the impulse was finite in length, and should be truncated after it reduced down to the level of the noise occurring prior to the impulse onset (i.e. plus and minus one least significant bit for this example using 8-bit data). 


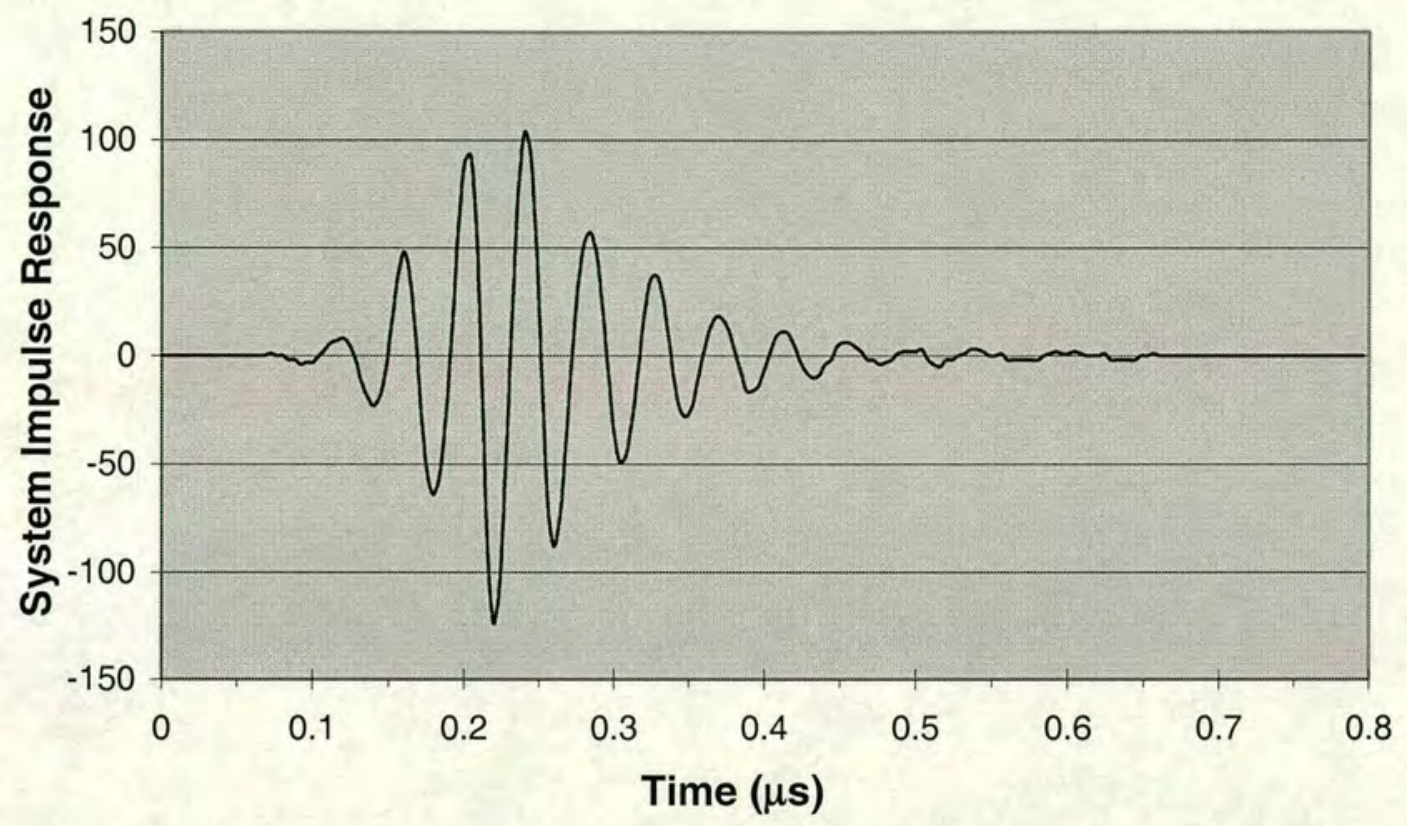

Figure 4.5 A measured system impulse response - for one IVUS system

\subsection{Design technique and theory}

The sigmoid based pseudo inverse filter design is based on the design of an inverse filter, but with reduced amplification of the smaller Fourier components. The amount that the smaller Fourier components are reduced by (relative to a classical inverse filter) is determined by a smooth cutoff function, that is a sigmoid function. Using a smooth cutoff function will cause the filter's frequency response to remain a continuous function. This is necessary because if the filter's frequency response were designed to be discontinuous (as with a hard cutoff) then the required number of filter taps would increase greatly. Further information about the design and original motivation for this filter, can be found in the original presentation of this filter, from H. Basil's Ph.D. thesis, [80] the relevant section of which is reproduced in Appendix I.

The filter is constructed in the frequency domain. Every Fourier component must be normalised prior to pseudo inversion. Each Fourier component of a system's impulse response is pseudo-inversed according to the sigmoid based equation shown in 
equation 4.1. After pseudo inversion, the filter taps are formed by conversion back into the time domain (using an inverse Fourier transform).

$$
\text { P.I. }(\omega)=\frac{\operatorname{Arg}\left(F^{*}(\omega)\right) \cdot \operatorname{Sig}(G \cdot(|F(\omega)|-T))}{|F(\omega)|+d}
$$

In equation $4.1, \mathrm{G}$ is a gain factor (typically 10 to 100 ), $\mathrm{T}$ is a threshold value, typically 0.01 to 0.10 , and $d$ is a small stabilising factor (typically 0.01 to 0.05 ). $F(\omega)$ must be normalised prior to applying equation 4.1 .

The threshold $T$ corresponds loosely to a half power or $3 \mathrm{~dB}$ point. More strictly, it defines the size of a Fourier component that will have a filter response that is half of what the filter response would have been for an inverse filter - as given by equation 4.2:

$$
|F(\omega)|=T \Rightarrow|P . I .(\omega)|=\frac{1}{2} \cdot \frac{1}{|F(\omega)|}
$$

The gain parameter $G$ defines how quickly the filter diverges from the inverse filter on either side of the threshold point.

In equation $4.1, \operatorname{Sig}(x)$ is a sigmoid function, with values ranging between 0.0 and 1.0. The sigmoid equation is shown in equation 4.3 and is plotted in Figure 4.6:

$$
\operatorname{Sig}(x)=\frac{1}{1+e^{-x}}
$$

The sigmoid function provides a smooth transition between an inverse filter for larger Fourier components and a matched filter for very small Fourier components. 


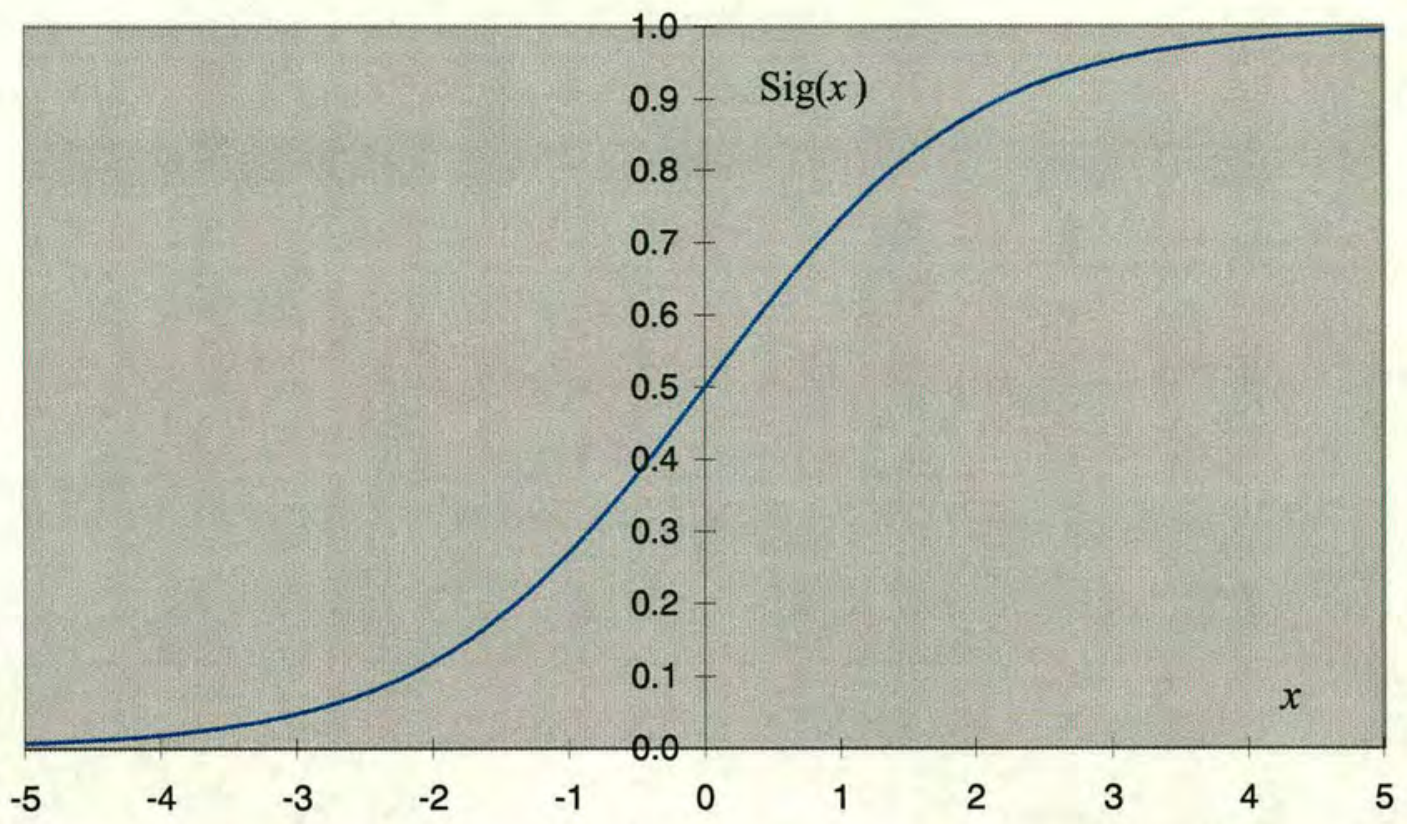

Figure 4.6 The sigmoid function

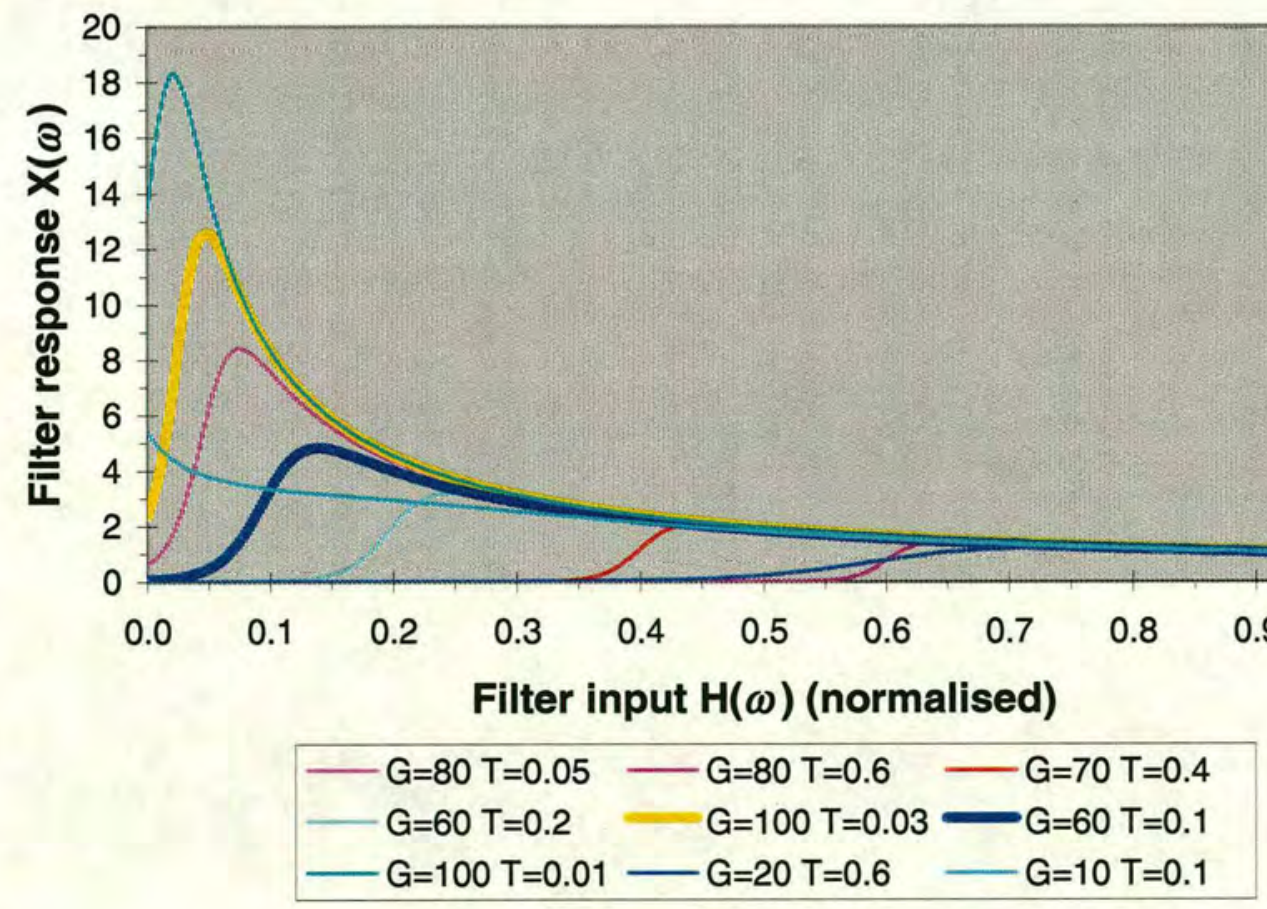

Figure 4.7 Sigmoid based pseudo inversion functions 
Varying the design parameters (particularly the threshold and the gain) yields differing pseudo-inverse functions. Figure 4.7 shows a variety of different sigmoid based pseudo-inverse functions.

Good results were obtained with design parameters of $\mathrm{G}$ between 50 and 150 , T between 0.01 and 0.1 , and $d$ between 0.01 and 0.05 . Using parameters within this range results in a filter that is sufficiently insensitive to noise for the IVUS data studied here. If the filter profile was well removed from an inverse filter, the image resolution improvement was too limited, and if the filter became too close to the profile of the inverse filter, the image noise became excessive. The above ranges were found to provide a good compromise between these two extremes.

\subsubsection{Modifications from previously published specification}

A modification to the previously published specification of the sigmoid based pseudo inversion process is now proposed. The motivation for this change is that when the offset $d$ is small, then small Fourier components are attenuated only slightly. This can be called the lip effect and it can be seen in Figure 4.8 and more clearly seen in the version shown in Figure 4.9. Both Figure 4.8 and Figure 4.9 show a sigmoid based pseudo inverse function with the same gain $G=80$, and the same threshold $T=0.05$. The values of the offset $d$ is different for each line plotted.

The lip effect becomes more important when the normalised Fourier components get smaller - and this will happen when using a higher resolution digitising process. This means that the lip effect is less noticeable with 8-bit data, but is much more noticeable with 12-bit data. If the ultrasound were sampled at 16-bits then the effect would be more noticeable again. 


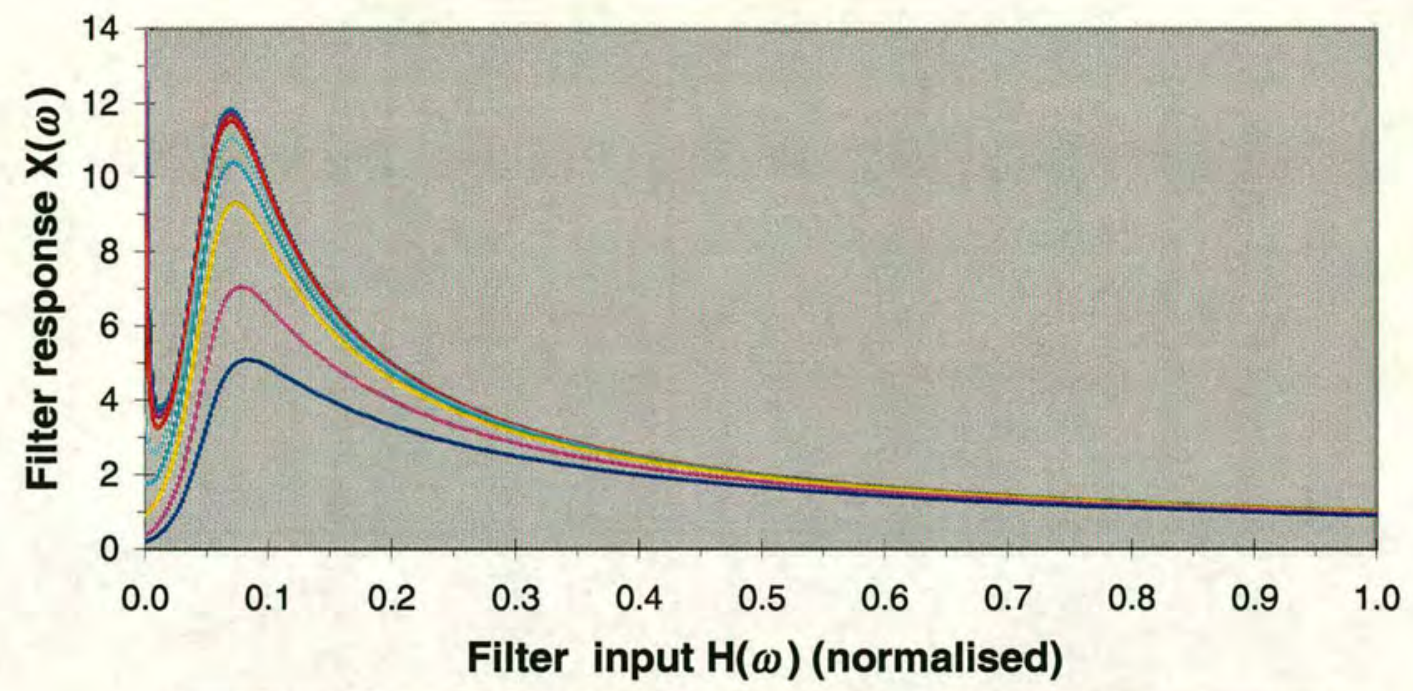

$-\mathrm{d}=0.0002-\mathrm{d}=0.0005-\mathrm{d}=0.001-\mathrm{d}=0.002-\mathrm{d}=0.005$
$\mathrm{~d}=0.01-\mathrm{d}=0.02-\mathrm{d}=0.05-\mathrm{d}=0.1$

Figure 4.8 Sigmoid based pseudo inverse functions ( $G=80$ and $T=0.05)$

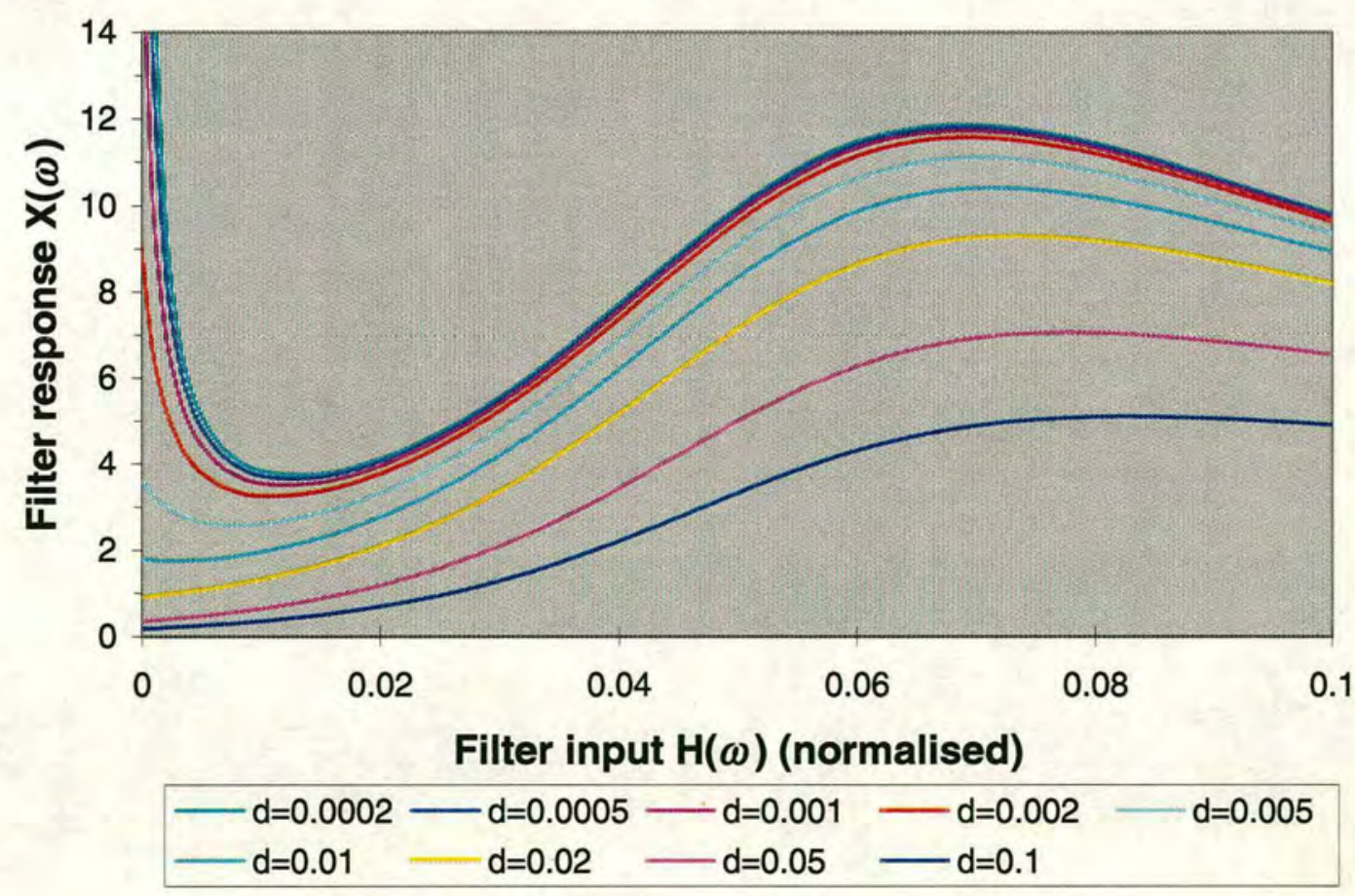

Figure 4.9 The lower end of some sigmoid based pseudo inverse functions. ( $G=80$ and $T=0.05$ ) 
In order to remove the lip effect as seen in Figure 4.9, equation 4.1 was modified so that small value Fourier components were truncated. It is desirable that the resulting pseudo inverse function should be a continuous function, and also give a gain of zero for Fourier components with a magnitude of zero. It was decided to truncate only those Fourier components of magnitude less than half the threshold $T$, and to truncate them in a manner similar to the matched filter - with linear interpolation of the function between the points $T / 2$, and 0 . The new equation is shown in equation 4.4, and is plotted in Figure 4.10:

$$
P . I .(\omega)= \begin{cases}\frac{\operatorname{Arg}\left(F^{*}(\omega)\right) \cdot|F(\omega)| \cdot \mid P . I .(T / 2)}{T / 2} & |F(\omega)|<T / 2 \\ \frac{\operatorname{Arg}\left(F^{*}(\omega)\right) \cdot \operatorname{Sig}(G \cdot(|F(\omega)|-T))}{|F(\omega)|+d} & |F(\omega)| \geq T / 2\end{cases}
$$

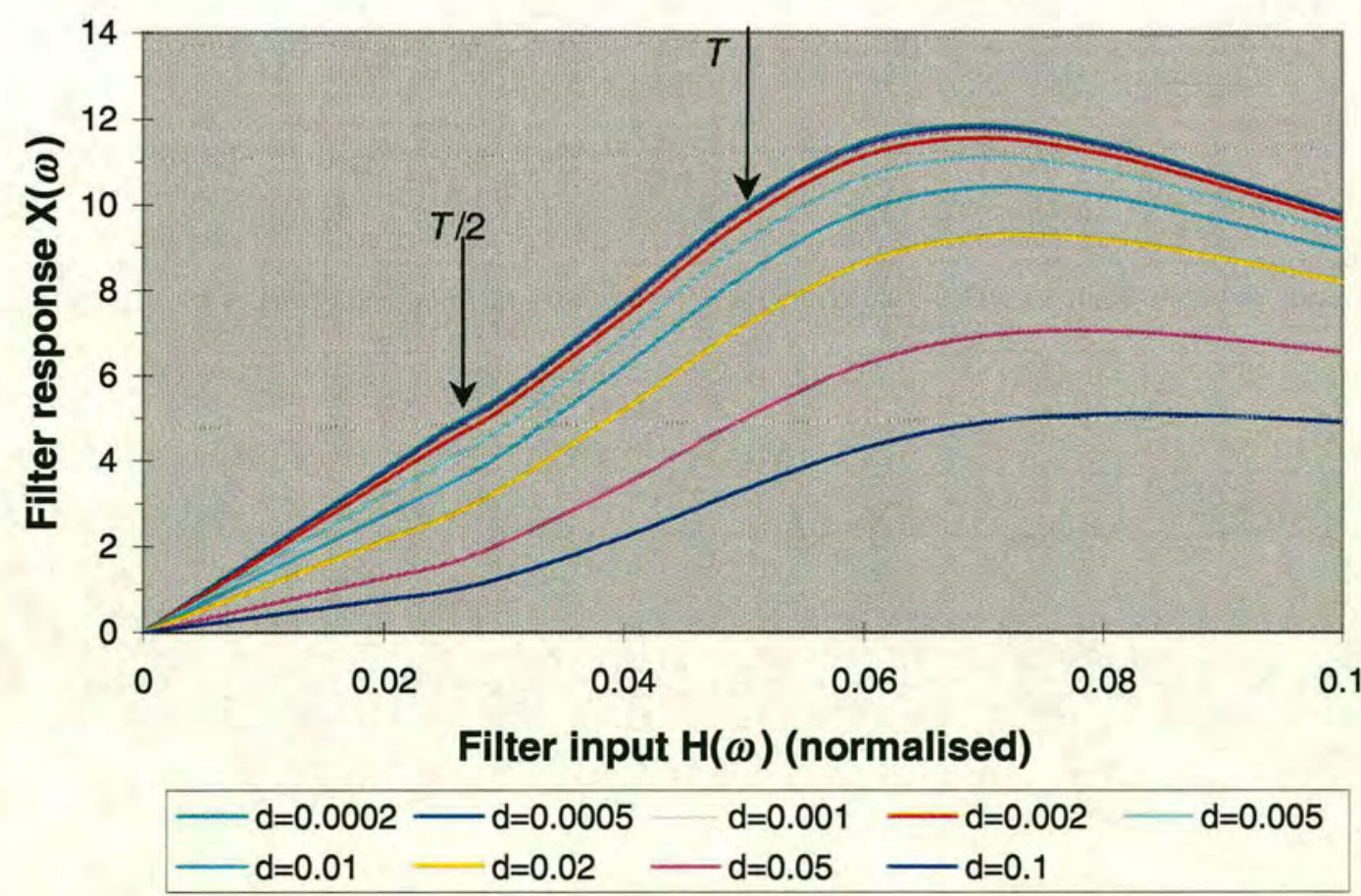

Figure 4.10 The lower end of some modified sigmoid based pseudo inverse functions. ( $G=80$ and $T=0.05$ ) 


\subsection{Example design}

To illustrate the design technique for a sigmoid based pseudo inverse filter, an example design will be performed. This is a design for an IVUS imaging system, whose measured impulse response (from a plane reflector) is shown in Figure 4.5.

\subsubsection{Nomenclature}

For the purposes of this thesis (and also the accompanying software), the following abbreviations will be used for sets of sigmoid based pseudo inverse filter parameters. Each abbreviation (or named filter) is a typical filter for this algorithm. The filters' parameters have been chosen so that when they are applied to ultrasound, they yield reasonable results while also being different enough to demonstrate the trade off between the inverse and matched filters.

\begin{tabular}{|l|c|c|c|}
\hline Given name & Gain $\boldsymbol{G}$ & Threshold $T$ & offset d \\
\hline Sigmoid PI1 & 60 & 0.1 & 0.05 \\
\hline Sigmoid PI2 & 80 & 0.05 & 0.03 \\
\hline Sigmoid PI3 & 100 & 0.03 & 0.02 \\
\hline Sigmoid PI5 & 100 & 0.01 & 0.02 \\
\hline
\end{tabular}

Table 4.1 Names assigned to parameter sets for sigmoid based PI filters

\subsubsection{Frequency domain conversion}

The first step is to perform an FFT on the impulse response. The length of the FFT is not critical. In this example the impulse response was zero padded until it was 2048 sample points long, although the actual impulse is about 146 samples in duration. The spectrum of the impulse response in this example is shown in Figure 4.11. 


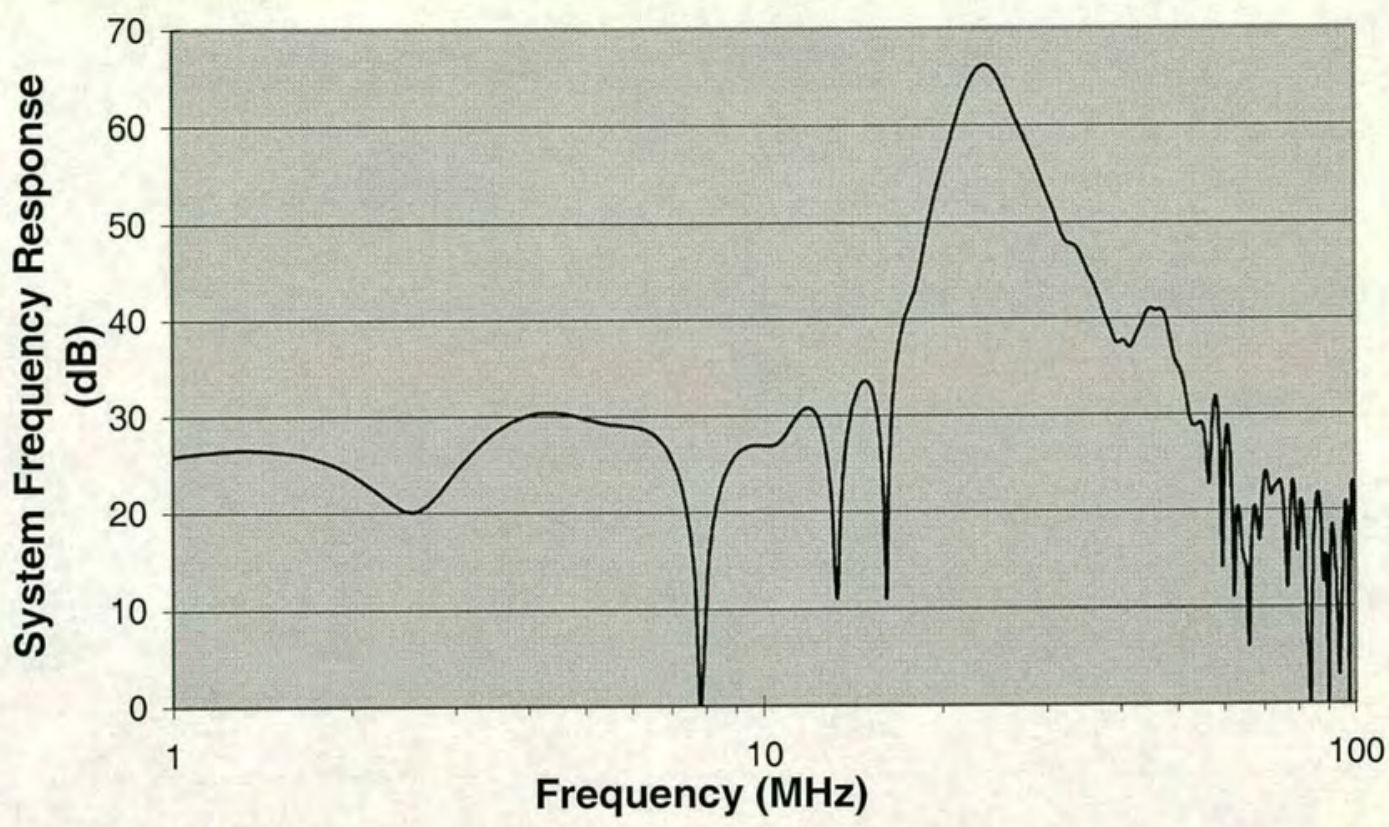

Figure 4.11 Frequency response of an IVUS system frequency response

After performing an FFT, every Fourier component must be normalised (with respect to the largest Fourier component). Thus the largest Fourier component will now have a magnitude of 1 .

\subsubsection{Pseudo inversion}

The sigmoid based pseudo inverse equation has three parameters that must be decided upon, the gain $G$, the inversion threshold $T$, and the offset $d$. For this example two filters will be designed, each with typical parameter values, which are defined as:

- $G=100, T=0.03, d=0.02,(=$ Sigmoid PI3);

- $G=60, T=0.1, d=0.05,(=$ Sigmoid PI1).

The first set of filter parameters has a lower threshold value, a higher gain, and also a smaller offset than the second set of parameters. Therefore, of the two resulting filters, the first will be closer to an inverse filter than the second. This can be seen 
clearly in Figure 4.7 where both the example filter functions are graphed (with the thicker lines).

The inversion performed here is done using both equation 4.1 and equation 4.4. The gain function of the two resulting filters is shown Figure 4.12 (for equation 4.1) and Figure 4.13 (for equation 4.4). Note that the gain function is close to an inverse within the passband", and is close to a level shifted matched filter outside the passband.

Note also that between 30 and $50 \mathrm{MHz}$, the two filters give vastly different responses. The filter Sigmoid PI1 is rolling off and attenuating the signal, where as the Sigmoid PI3 filter is passing the signal, and is still giving a reasonable approximation to an inverse filter. This causes the two filters to have different effective passbands, and yield different results when applied to ultrasound images.

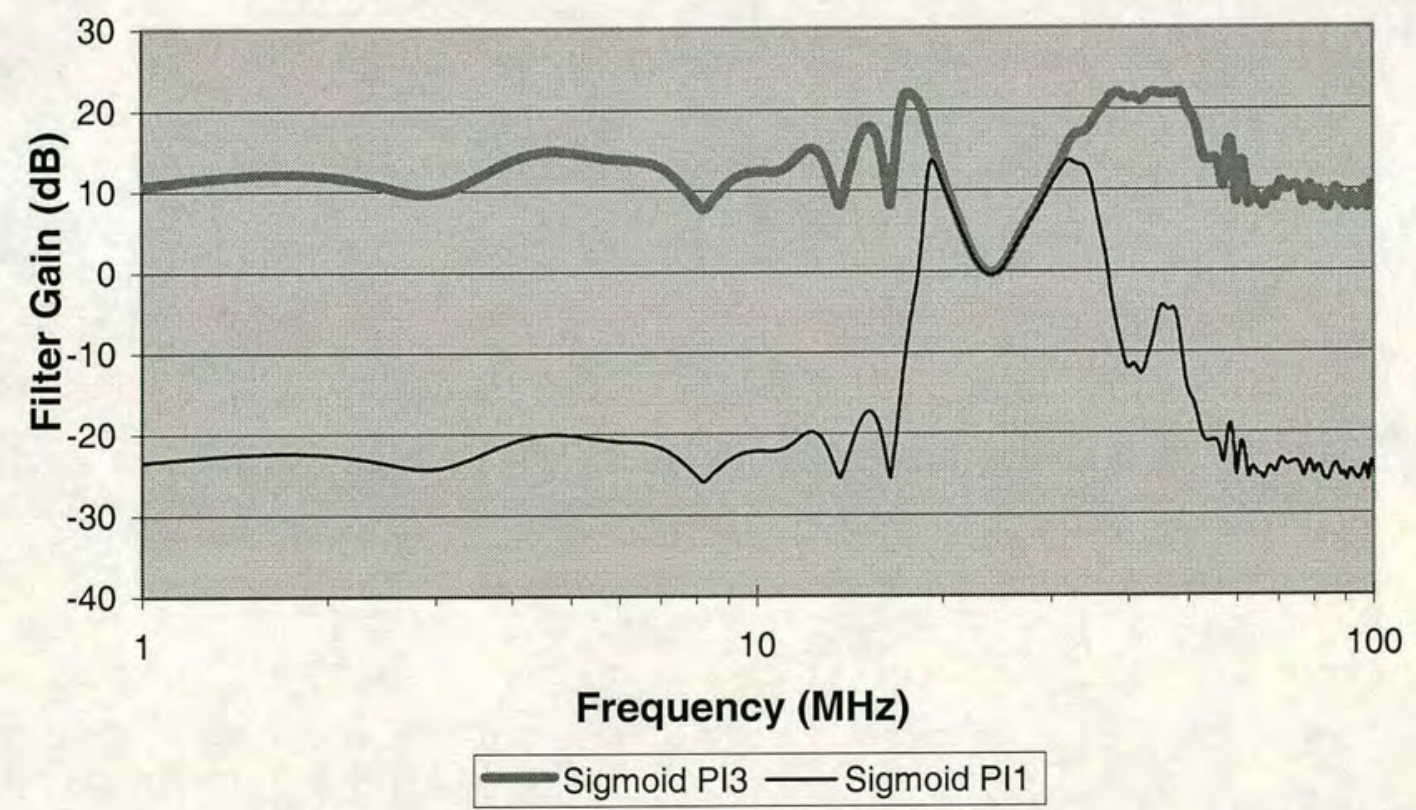

Figure 4.12 Filter gain function for two typical sigmoid pseudo-inverse filters

"The two example filters have different effective passbands. 


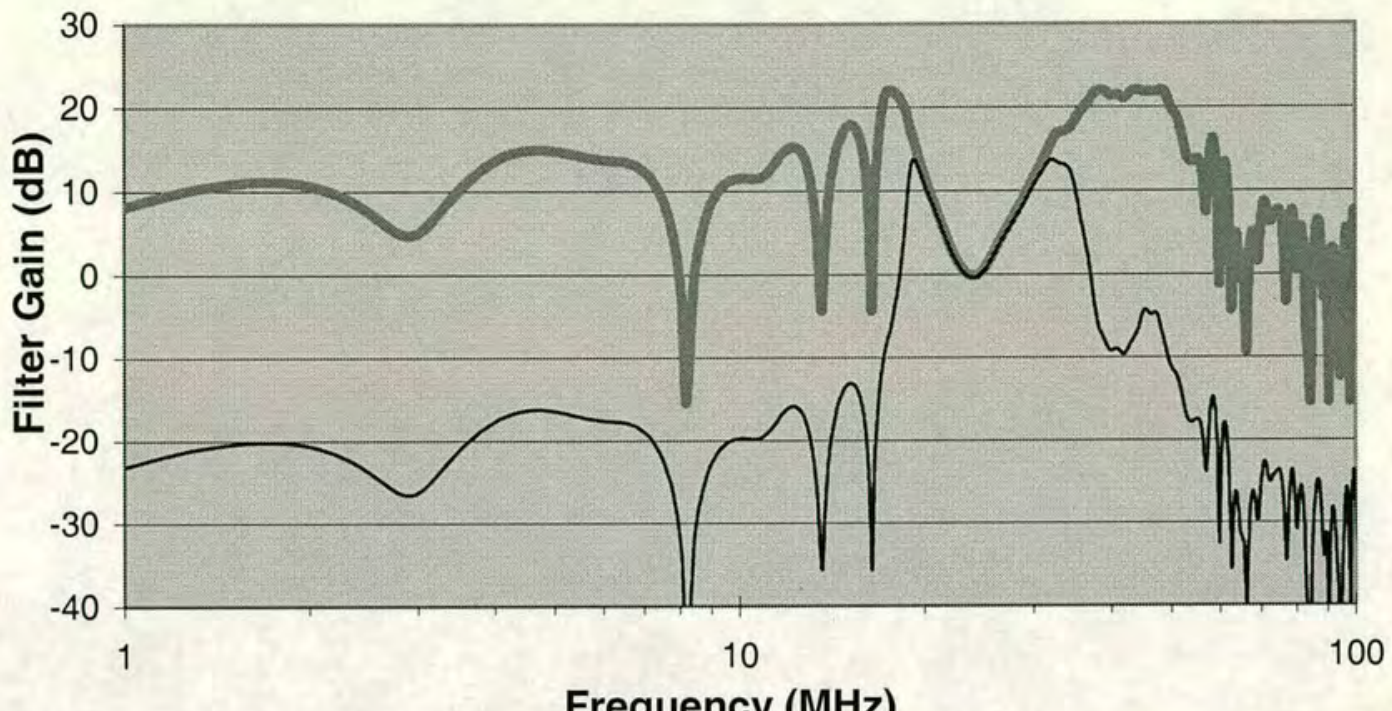

Frequency $(\mathrm{MHz})$

Sigmoid PI3 — Sigmoid PI1

Figure 4.13 Modified filter gain function for two typical sigmoid pseudo-inverse filters

\section{Modification for the lip effect}

In this case, the lip effect is minimal due to the selection of filter parameters. Also the digitised data was only 8-bit. Thus implementing the modified sigmoid pseudo inverse of equation 4.4 in this case will have a small effect only. In fact, in the second example design, the modification will actually increase the response of some Fourier components. The modified filter gain functions are shown in Figure 4.13.

\subsubsection{Conversion back to time domain - making the filter taps}

After the Fourier transform is pseudo inversed, an inverse FFT is performed to take the signal back into the time domain. The result of the IFFT will be the filter taps for the designed filter. For the two example filter designs, the resulting filter taps are shown in Figure 4.14. The filter taps have been normalised. Note that the two filters are plotted on separate axes for clarity. 


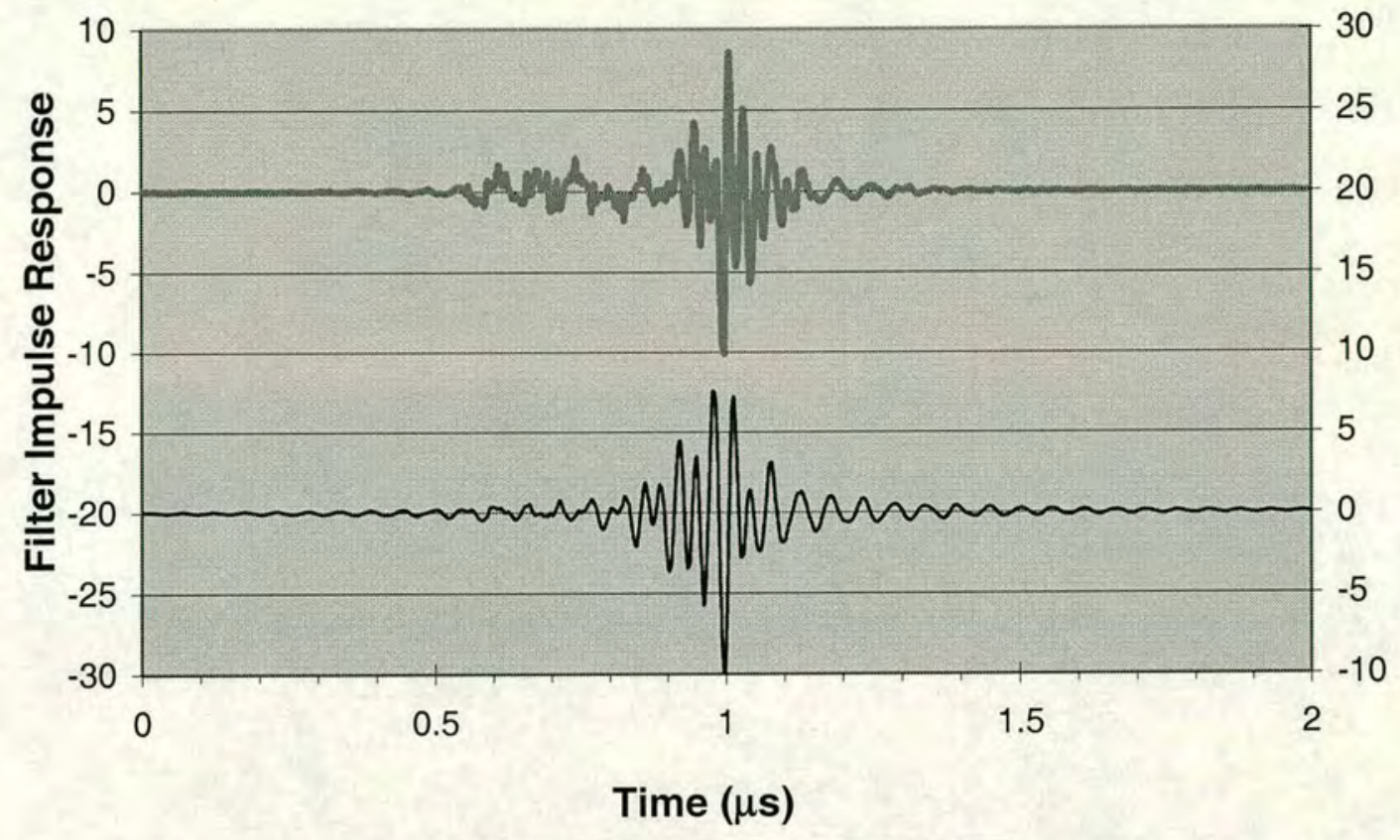

Sigmoid PI3 — Sigmoid PI1

Figure 4.14 Resulting filter impulse responses for two typical sigmoid pseudo-inverse filters

The filter taps shown are the result of the modified sigmoid based pseudo inverse function given in equation 4.4. However, the choice of filter design parameters in this case means that the modification to the sigmoid based pseudo inverse function is minimal. The graph of filter taps would look the same if equation 4.1 had been used, as the differences are too small to observe in this format.

\subsubsection{Design analysis}

\section{Transfer functions}

The transfer function of the two example filter designs are shown in Figure 4.13 (frequency domain) and Figure 4.14 (time domain). Note that the filter plotted higher is for the filter Sigmoid PI3 which has a wider passband than the filter Sigmoid PI1. The wider passband causes the filter taps (or the filter's impulse response) to have more high frequency oscillations. 


\section{Resulting power spectra}

It is useful to analyse the output of the filter, given the system impulse response as an input (with no noise added). Although this is an artificial test, it is useful for verifying the algorithm. The output can be analysed in the time domain and the frequency domain.

The output power spectrum is shown in Figure 4.15. Clearly it is now closer to the spectrum yielded by an inverse filter - especially within the passband. Outside the passband however the spectrum shape is largely unchanged, although its level may change. This shows that the filter has acted like an inverse filter within the passband, outside the passband it has acted a bit like a level shifted matched filter.

It can be clearly seen that the filter Sigmoid PI3 has yielded a significantly wider passband that the Sigmoid PI1 filter. The region from 30 to $50 \mathrm{MHz}$ is within the passband of Sigmoid PI3, but not within the passband of Sigmoid PI1.

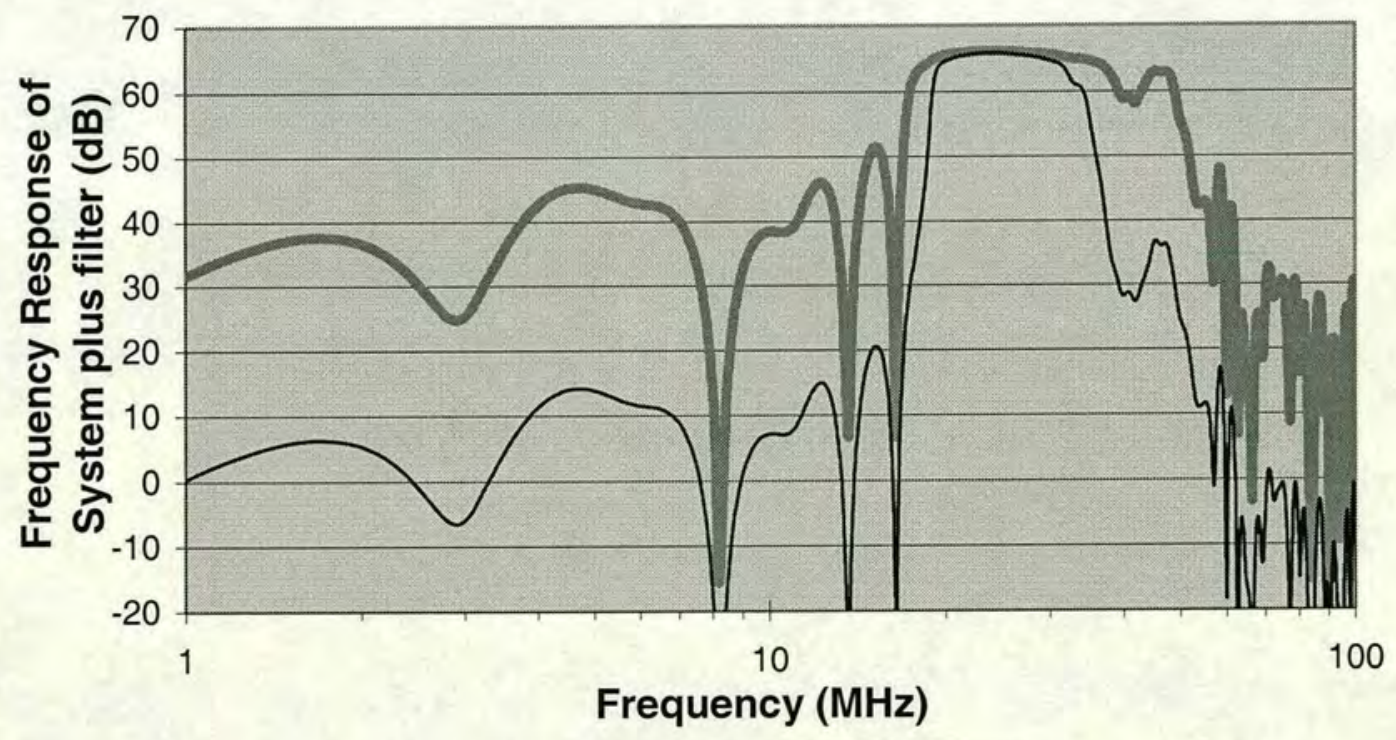

Sigmoid PI3 — Sigmoid PI1

Figure 4.15 Resultant power spectra after filtering the system impulse response with two typical sigmoid pseudo-inverse filters 


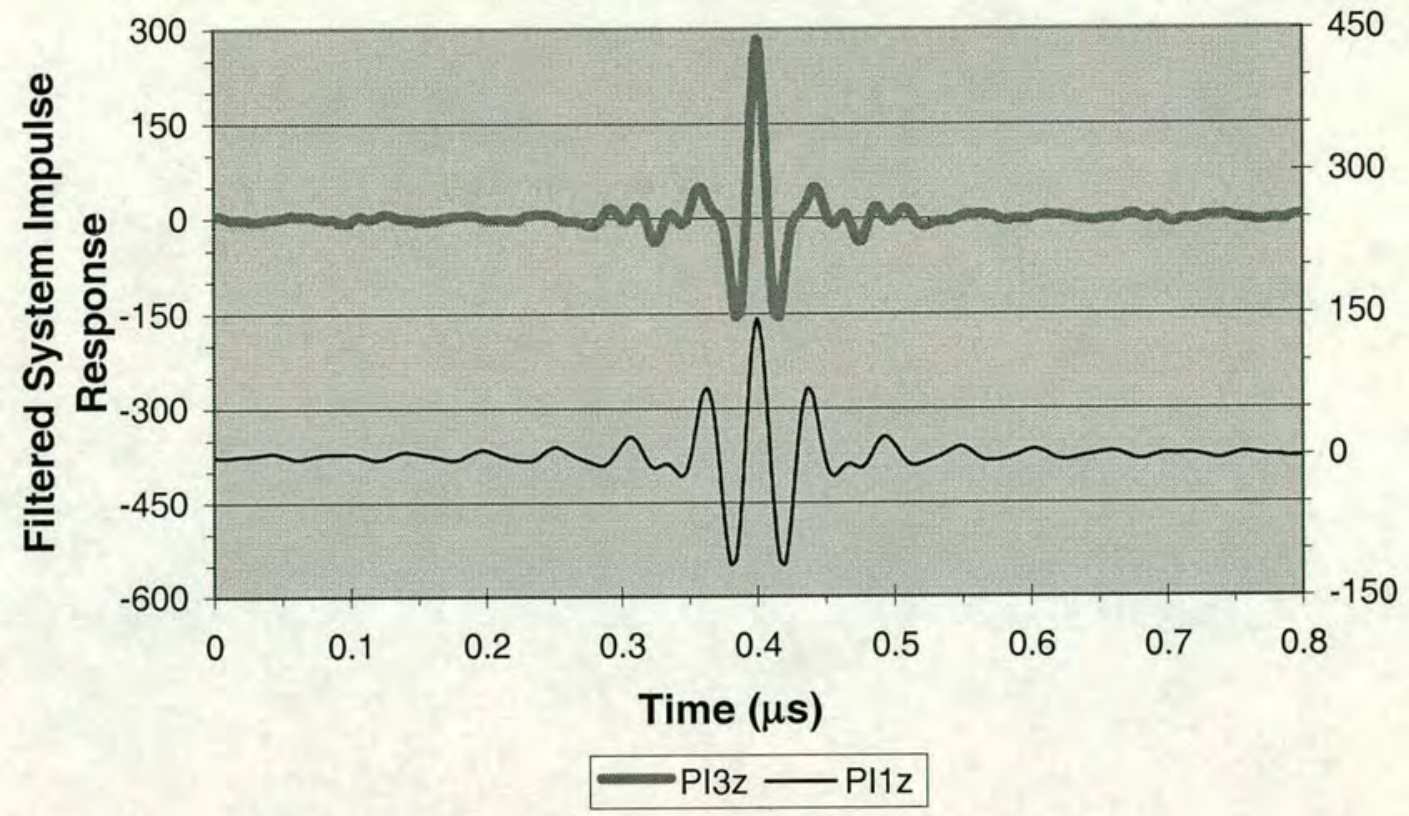

Figure 4.16 Resultant signal after filtering the system impulse response with two typical sigmoid pseudo-inverse filters

The output signal in the time domain is shown in Figure 4.16. The duration of the pulse has been reduced. This shows that the filter has acted as a pseudo inverse filter. The resulting pulse is longer or shorter depending on how close the designed filter is to an inverse filter. Note that this example is noise free, and the introduction of noise will degrade the filters - with more degradation for filters that are close to inverse.

After filtering the system impulse response with two example sigmoid pseudoinverse filter designs, it is clear that the filter is indeed acting as a pseudo inverse filter.

\subsection{Windowing effects on filter taps}

All Fourier transforms were performed with a length of either 2048 or 4096 points (mostly with 2048). This means that after inverse Fourier transforming and obtaining filter taps, the taps were up to 2048 points long. However most of these points were either zero or very close to zero. 
The filter tap series were truncated, and although this will alter the filter's frequency response, in practice the effect is minimal - provided the series is not too short. Figure 4.17 shows the filter gain function designed in Figure 4.13 before and after it has been truncated by applying a Hann window to the taps. The length of the Hann window was: (a) double the length of the impulse response, and (b) the same length as the impulse response. Using a Hann window three times the length of the impulse response yielded a gain function very similar to the original gain function (too similar to differentiate on this graph).

From Figure 4.17 we can see that truncating the filter taps to the same length as the impulse response causes a significant change in the filter's gain function. However truncating the taps to two or three times the impulse response length causes only minor changes.

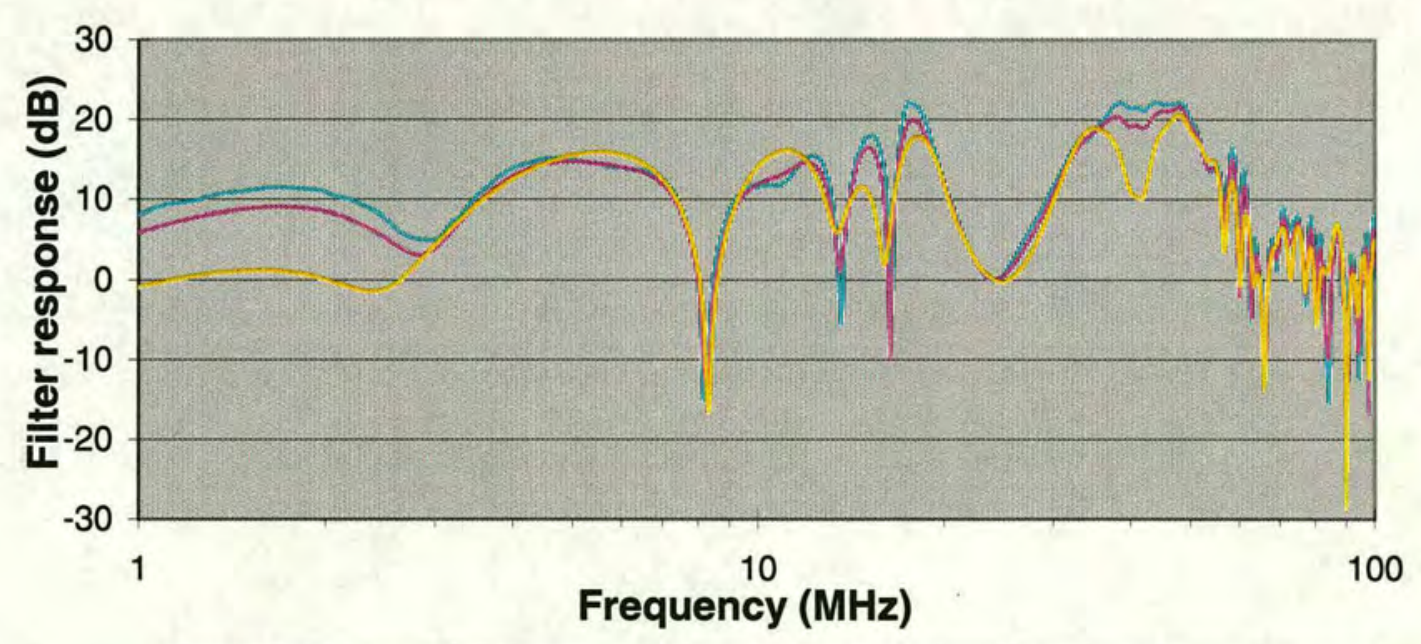

Unwindowed
Windowed with a Hann window twice the length of the impulse response.
Windowed with a Hann window the same length of the impulse response.

Figure 4.17 An example sigmoid based pseudo inverse filter, truncated with varying length Hann windows 


\subsection{Comparison with other filters}

It is useful to compare the sigmoid based pseudo inverse functions with the equivalent functions that define other filters such as the matched filter, the inverse filter, the phase correction filter, the square root filter and Wiener MMSE filters. Figure 4.18 gives a comparison with filters described by $\mathbf{X}(\omega)=\mathbf{H}(\omega)^{(\mathrm{n}-1) / \mathrm{n}}$. Figure 4.19 gives a comparison with some Wiener MMSE filters.

It can be clearly seen that each sigmoid based filter lies between the matched filter and the inverse filter. For frequencies with low signal level, the sigmoid based filters will give less gain than the inverse, $4^{\text {th }}$ root, and square root filter. Often they will give less gain than even the phase correction filter (i.e. they will actually attenuate these frequencies).

Sigmoid PI1, Sigmoid PI2, Sigmoid PI3, and Sigmoid PI5 are all defined in Table 4.1.

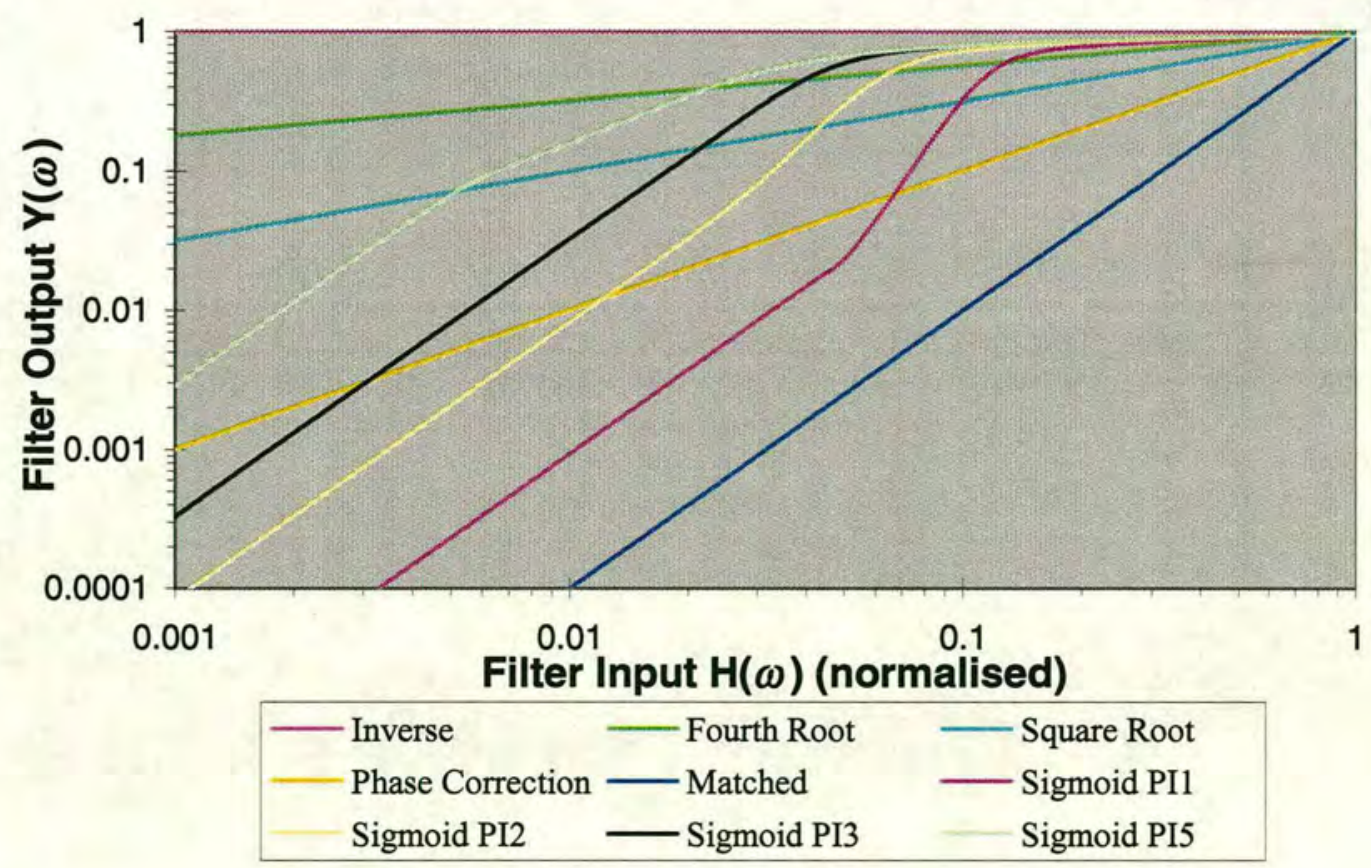

Figure 4.18 Sigmoid filter output functions for a noise free impulse as input, compared with $X(n-1) / n$ filters 


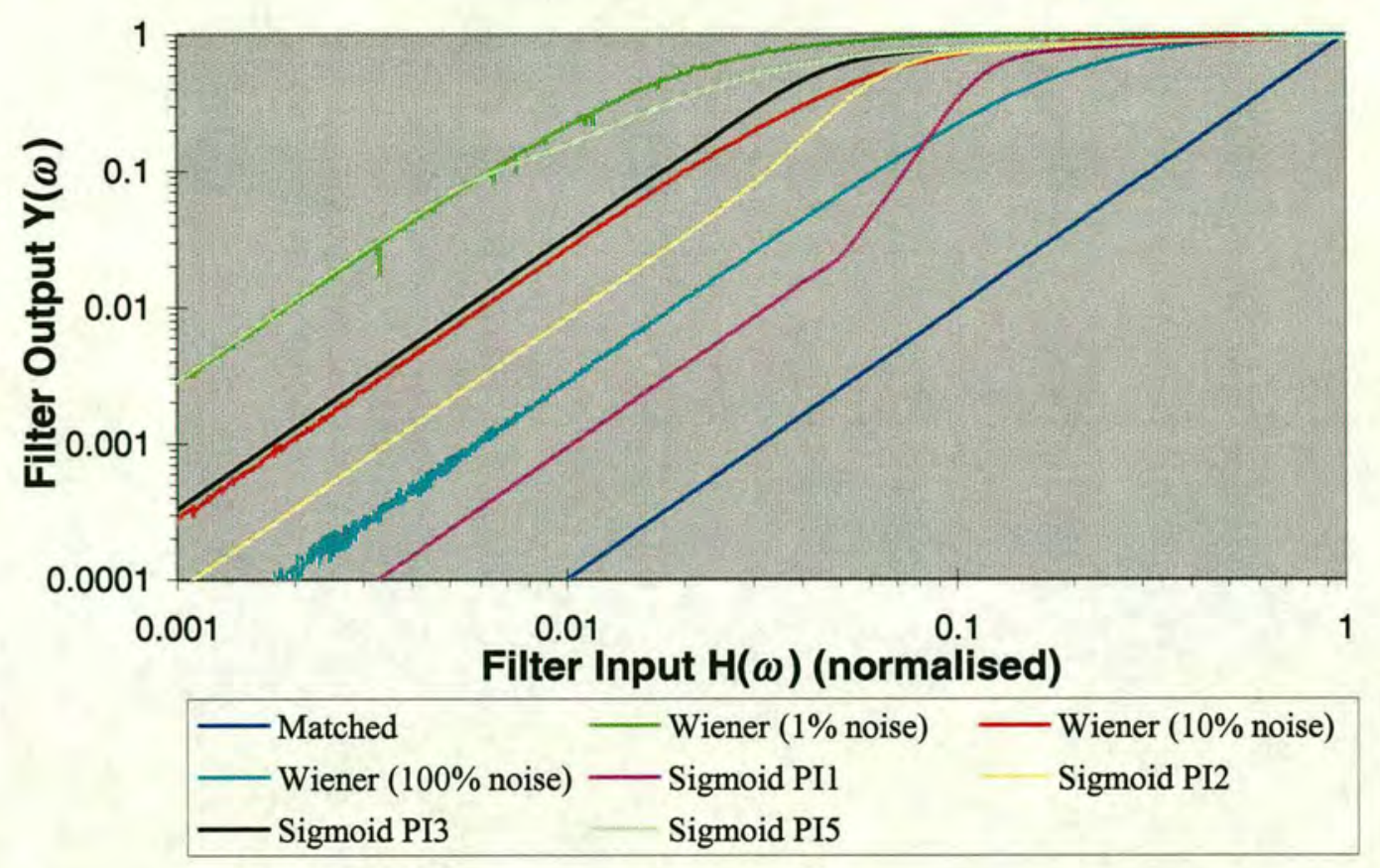

Figure 4.19 Sigmoid filter output functions for a noise free impulse as input, compared with Wiener filters

It can be seen that the sigmoid based pseudo inverse filters are able to produce functions that are similar (though not identical) to the Wiener filter. All the curves in Figure 4.19 resemble the inverse filter for large frequency components (right hand side of graph) and have the same gradient as the matched filter for smaller frequency components (left hand side of graph).

\subsection{Conclusion}

The two different example designs presented illustrate how the sigmoid based pseudo inverse filter can be used to produce filters that trade off between the inverse filter and the matched filter.

Some degree of care is required in the choice of the filter parameters, but the modified algorithm presented will allow a much wider range of acceptable values. 
The filter described in this chapter is implemented in the software on the accompanying CD (It is implemented both with, and without the proposed modification). Instructions for the operation of the program that implements the design and application of this filter is described in Appendix C. 


\section{Chapter $5 . \quad$ UltRAsound Setup}

\subsection{Introduction}

Multiple sets of ultrasound data and images are analysed in this thesis. They come from several principal sources:

- intravascular ultrasound collected in vitro from human coronary arteries;

- intravascular ultrasound collected in vivo from rabbits;

- near perfect plane reflectors imaged with the intravascular ultrasound system;

- a phantom imaged with a phased array, general purpose ultrasound imaging system;

- near perfect plane reflectors imaged with a phased array, general purpose ultrasound imaging system.

This chapter details the data collection systems and procedures used in acquiring this data. The near perfect plane reflectors were imaged with the same transducers as the other images (for both intravascular and phantom).

\subsection{IVUS data collection system}

This section outlines the setup that was used to collect IVUS data in vitro, in vivo, and with perfect reflectors [21]. 


\subsubsection{In vitro setup}

The IVUS data collection in vitro was performed with a setup as shown in Figure 5.1. A saline solution is flowing through the artery. This is needed to ensure that there is no air in the system due to the high acoustic impedance of an air-liquid or air-tissue interface.

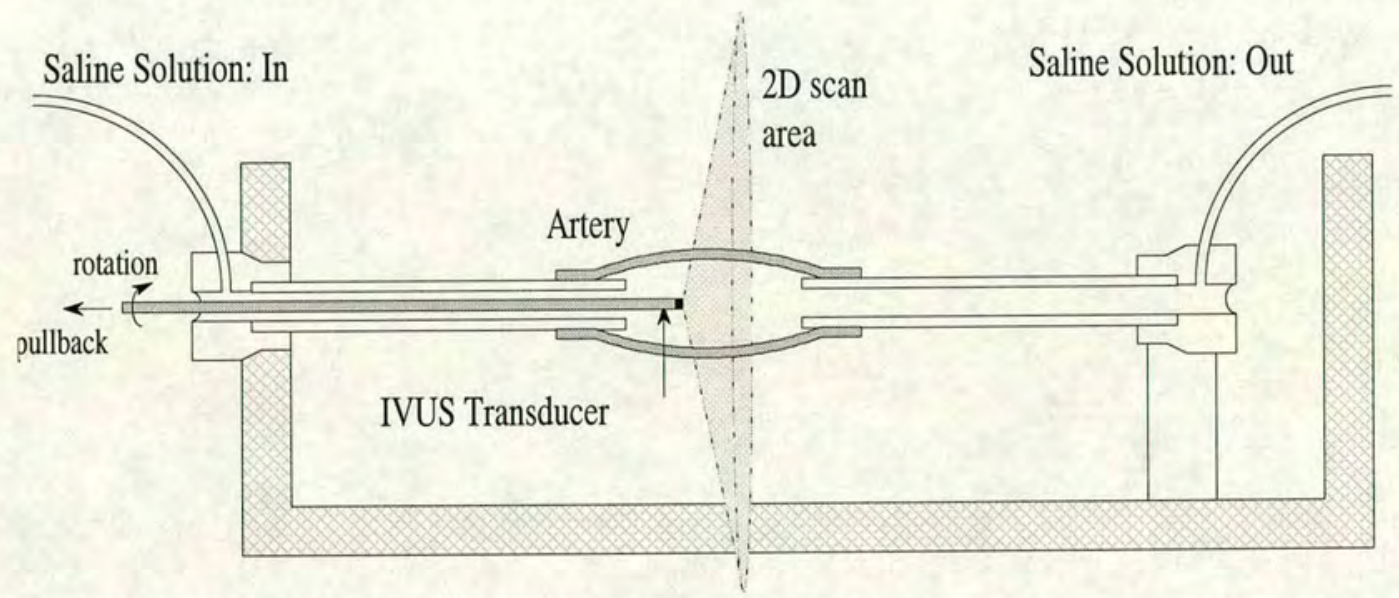

Figure 5.1 In vitro setup for IVUS data collection

\section{The transducers}

The transducers used for the in vitro IVUS data collection were made by the Boston Scientific Company, Watertown, MA, USA. They are 3.5 French $(0.66 \mathrm{~mm}$ diameter), slightly forward looking (about $12^{\circ}$ ), and operate at $30 \mathrm{MHz}$. i.e. $30 \mathrm{MHz}$ is the nominal frequency. The transducers have a bandwidth of approximately 15 $\mathrm{MHz}$ centred around the nominal frequency. They are unfocussed so that the beam does not diverge too much over a wider axial range.

Due to the invasive nature of IVUS imaging and the need for sterility, IVUS transducers are never used on more than one patient in vivo. After in vivo use, they can however be flushed with water and later reused in vitro, and also for perfect reflector scans. In this case, the transducers can be repeatedly reused. 


\section{The scanning format}

The transducer obtains a 2D image at many locations as it is pulled back through the artery. After the transducer obtains a $2 \mathrm{D}$ image, it is pulled back by a controllable distance $(0.2 \mathrm{~mm}$ in this case), and a subsequent $2 \mathrm{D}$ image is obtained. In each dataset, there will be up to 200 pullback positions, (or $2002 \mathrm{D}$ images) covering a distance of $4 \mathrm{~cm}$.

As the transducer scans each $2 \mathrm{D}$ image, it rotates through $360^{\circ}$, in steps of $1.5^{\circ}$, controlled by a stepping motor. This means that 240 different directions will be scanned. These 240 1D scans are then combined to form a $2 \mathrm{D}$ image. This is illustrated in Figure 5.2. Each 1D scan is often called a scanline. One 2D image is constructed for each pullback position.

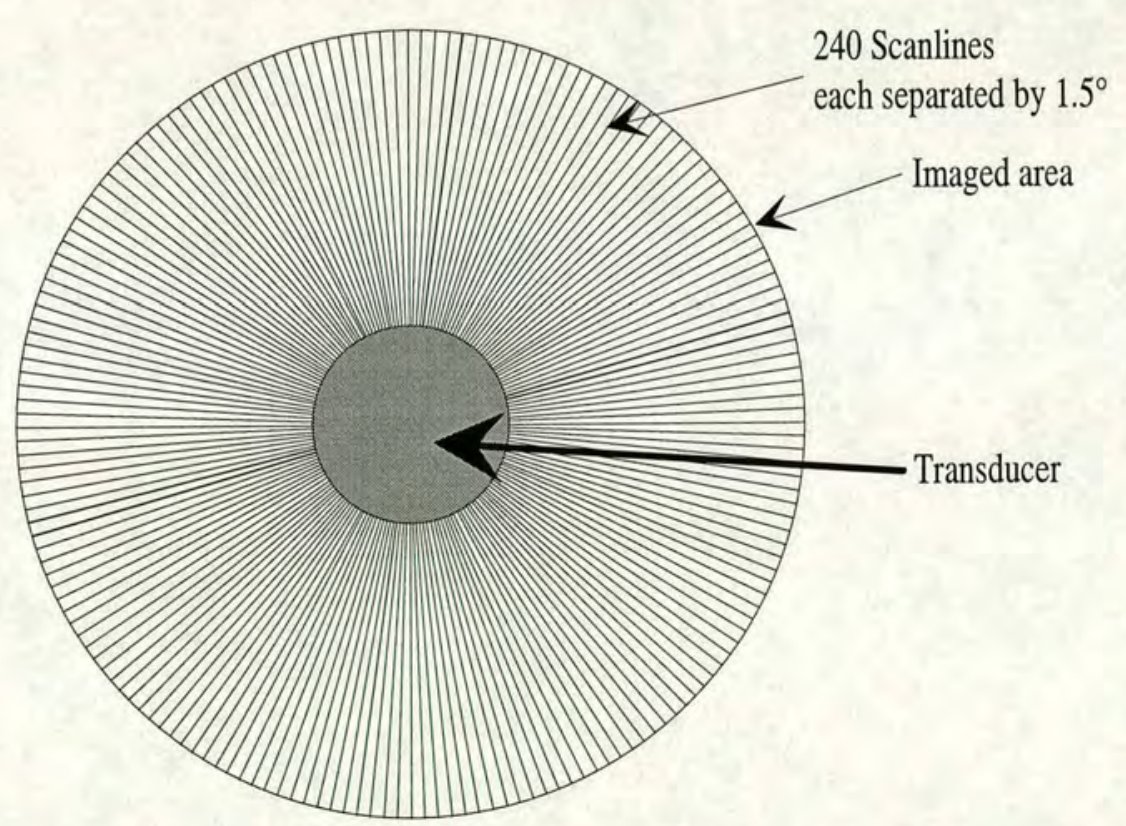

Figure 5.2 Format of IVUS $2 D$ scan constructed from $1 D$ scanlines

In order to construct each 1D scanline, the transducer sends out an ultrasound pulse, and then receives back the reflected signal. The received signal is amplified and digitised with no further processing. It is important to note that the digitised signal was not demodulated to baseband, it was recorded as an RF signal. 


\section{The stored image format}

The received ultrasound signal is digitised at $250 \mathrm{MHz}$ (or one sample every $4 \mathrm{~ns}$ ), with 2048 samples per scanline. Each sample is digitised with a resolution of 8 bits. Initially the data from each $2 \mathrm{D}$ image is digitised and is available as a continuous stream of 8 bit words. This data then has a header attached to it, to produce a greyscale bitmap. Each 2D image is then stored permanently as a greyscale bitmap; which represents a (geometrically distorted) B-mode image.

The bitmap header is defined such that every row of the bitmap corresponds to one 1D scanline within the image [111]. Therefore the bitmaps have dimensions of 240 rows by 2048 columns. An example of this type of image is shown in Figure 5.3. Note the large ringdown signals at the left of the image.

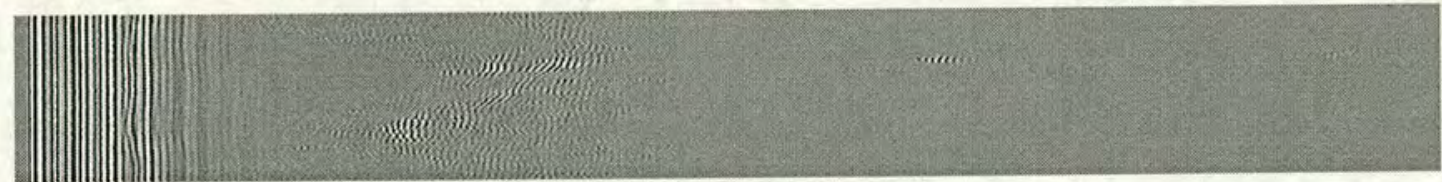

Figure 5.3 Raw IVUS data in bitmap format

Within each row of the bitmap (e.g. Figure 5.3), we can consider that the samples are positioned on a time axis - with time increasing from left to right. Alternatively we can consider that the samples are positioned on a distance axis (i.e. distance from the transducer). Either interpretation is valid, although only the time axis interpretation is completely accurate; the distance interpretation is only an approximation due to the speed of sound varying in different mediums.

The data in each 2D scan image can be scan converted (Hilbert transformed, log transformed and geometrically transformed) to produce an image of the artery at the given pullback position, as shown in Figure 5.4. 


\subsubsection{In vivo setup}

The in vivo IVUS data presented and analysed in this thesis is not from human patients; it is exclusively from rabbits. The images come from the coronary arteries of the rabbits. The rabbits had no arterial disease but had significant amounts of lipid build-up inside the arteries as a result of being fed a high fat diet. These rabbits were bred (and imaged) for research purposes at the Western General Hospital in Edinburgh.

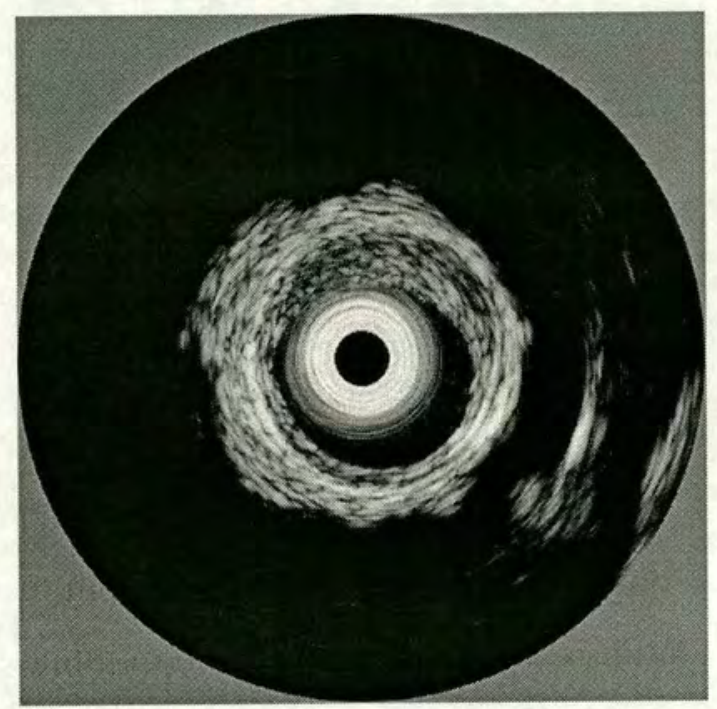

Figure 5.4 IVUS data after being processed for display

Although typical human patients would have significant arterial disease (such as atherosclerosis) the rabbit arteries are still able to provide a good test case for IVUS imaging. Importantly, the rabbit arteries are large enough that most of the ultrasound reflected from the artery does not lie in the ringdown region of the image.

The data format and transducer operation was identical to the in-vitro setup, except that the path of the transducer during pullback was (probably) not a straight line, and the data recording was ECG gated so that the recordings always occurred on the same phase of the cardiac cycle. 
The main set of images presented in this thesis come from the aorta of a rabbit, starting from the renal artery branch point. After a pullback covering 2 to $4 \mathrm{~cm}$, a coil was inserted to cause a thrombus (blood clot) to develop. Images were taken during this period but are not presented here. A full pullback was repeated after the development of the thrombus.

\subsubsection{Perfect reflector setup}

The near perfect reflector used with the IVUS transducer was a crown glass block. This makes a near perfect plane reflection. The block was optically flat to within $1 / 27$ th of a wavelength (at the nominal ultrasound frequency of $30 \mathrm{MHz}$ ) and was approximately $2.5 \mathrm{~cm}$ square, and $1.5 \mathrm{~cm}$ deep. The setup is shown (viewed from above) in Figure 5.5.

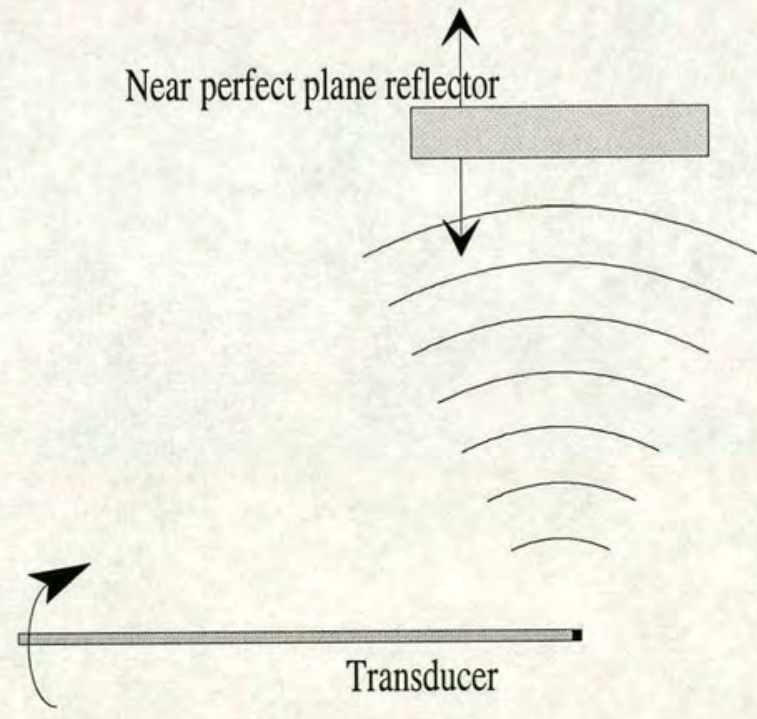

Figure 5.5 Setup for imaging a perfect reflector (IVUS transducer)

The perfect reflector image was obtained with the same IVUS transducers that were used for each IVUS dataset. The transducer rotates through a full circle, obtaining 240 scan-lines through the rotation. For collecting the perfect reflector data the transducer was still rotated through $360^{\circ}$, therefore only a small number of the 240 scan lines will have received a reflection from the perfect reflector. 
The following parameters could be adjusted when obtaining a perfect reflection:

- the distance from the transducer to the perfect reflector;

- the gain of the system prior to digitisation.

The system gain had to be adjusted so that the signal would not saturate the digitiser. (In some cases the digitiser did saturate).

It was not necessary to have the transducer directed exactly perpendicular to the reflector. (In medical imaging, the surfaces are never perpendicular). Acceptable perfect reflections could be obtained for quite a wide variety of angles (approximately up to 15 degrees or 10 scan-lines either side of perpendicular). This is due to the width of the reflector $(2.5 \mathrm{~cm}$ square), and its distance from the transducer (ranging between 3 and $8 \mathrm{~cm}$ ).

\subsection{Phased array (phantom) data collection}

The ultrasound system used for this imaging was an Acuson 128XP/10, together with a Tomtec machine to obtain 3D scans by mechanically rotating the transducer. A separate PC was used to digitise the received signal.

The phantom and transducer were arranged as shown in Figure 5.6. The phantom was fully contained within the imaged area of the transducer.

\subsubsection{In vitro phantom setup}

\section{The transducer}

The transducer used was supplied with the Acuson $128 \mathrm{XP} / 10$. It had a 128 element, 1D phased array, and gave a viewing angle of $77^{\circ}$. It uses 131 radial scanlines to construct each 2D image (a central line plus 65 more each side of centre). Thus the scanlines are separated by $0.59^{\circ}$. The transducer used had an adjustable nominal 
operating frequency. For all data presented here, the frequency was set to $2.5 \mathrm{MHz}$. Due to commercial secrecy, no further information is available on the details of the transducer.

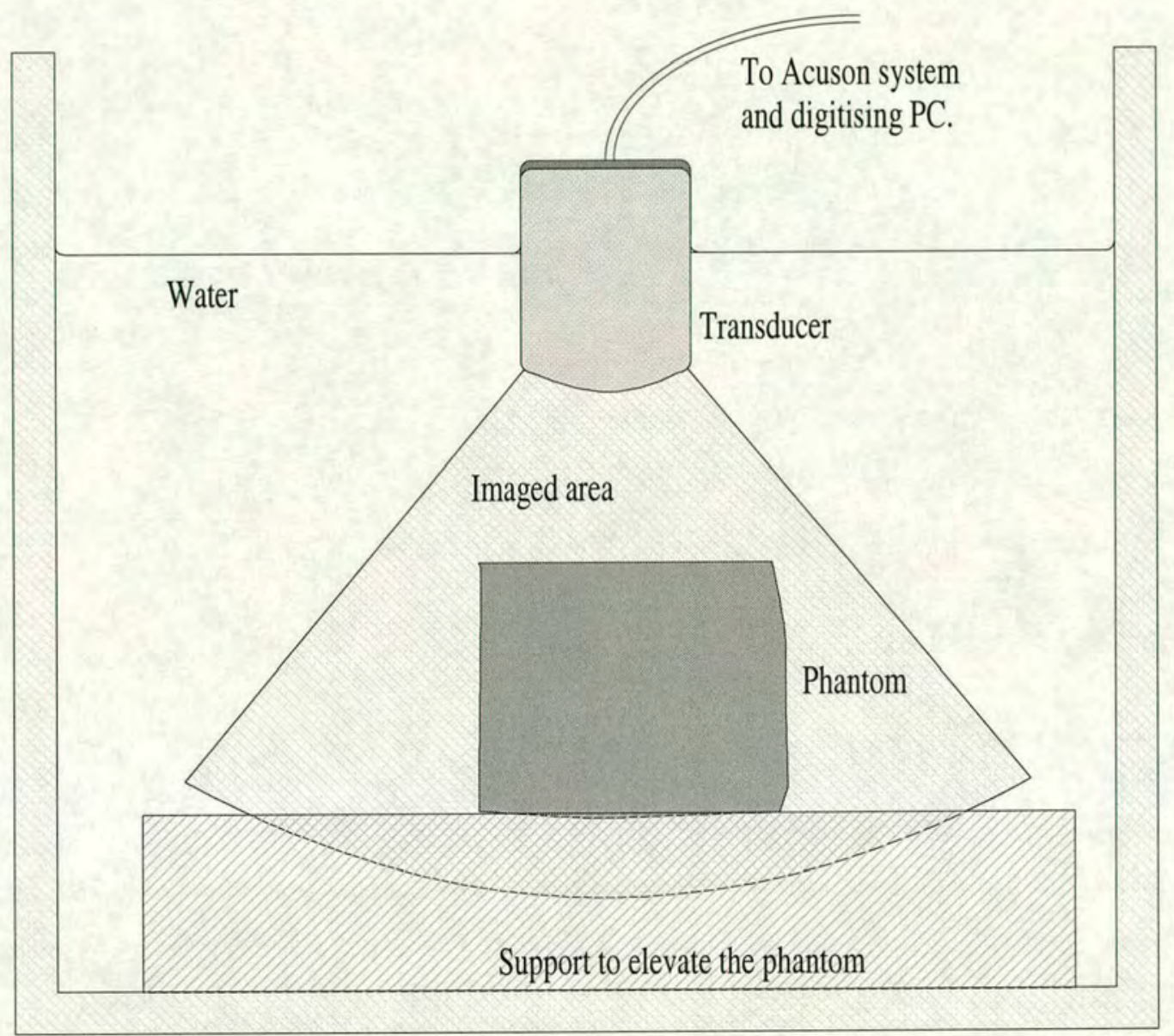

Figure 5.6 Setup of phantom for data collection

\section{The scanning format}

As the Tomtec machine rotated the transducer, successive $2 \mathrm{D}$ images were obtained. The Tomtec rotational device was set up to rotate through a half circle $\left(180^{\circ}\right)$ in 36 steps. ( $5^{\circ}$ for each step). Due to the symmetry of the imaged area in each $2 \mathrm{D}$ scan, it is sufficient to rotate the transducer through $180^{\circ}$ only, to obtain a full $3 \mathrm{D}$ volume scan. Any two scans that are $180^{\circ}$ apart will be images of the same area (although the 
image will be reversed). Because the phantom does not move with time, any scans $180^{\circ}$ apart will be identical (except for noise and image reversal).

For each 1D scan, the ultrasound signals received by the phased array were delayed, summed, and amplified, inside the Acuson 128XP/10, and were then sent to a PC, which digitised the signals. The delay and sum steps correspond to receive beam steering and focussing. Repeating this for 131 different beam directions constructs a $2 \mathrm{D}$ image. The digitised signal was not demodulated to baseband, it was recorded as an RF signal.

\section{The stored image format}

The data was digitised by a separate PC at $20 \mathrm{MHz}$ (or $50 \mathrm{~ns}$ ), with 4096 samples per scanline. Each sample is digitised with a resolution of 12 bits.

Initially the data from each $2 \mathrm{D}$ image is digitised and is available as a continuous stream of 16 bit words. Each 16 bit word contains 12 bits of data, followed by 4 padding zeros. Since each 2D image contains 131 scanlines, it follows that every 2D image consists of 1073152 bytes $(131 \times 4096 \times 2)$.

The data is presented from the digitising $\mathrm{PC}$ as a continuous block of data, with successive $2 \mathrm{D}$ images appended to each other. There was also a partial 2D image appended at the end, which was discarded. The data is firstly split into sections that are each 1073152 bytes long (i.e. split into individual 2D images).

Each 2D image is then manipulated by taking each 12 bits of data and padding it into a 24 bit word. The 24 bit data then has a header attached to it, to produce a truecolour (24 bit) bitmap. The bitmap header is defined such that every row of the bitmap corresponds to one 1D scan within the image. Each successive row is a successive 1D scan. Therefore the bitmaps have dimensions of 131 rows by 4096 columns. An example of this type of image is shown in Figure 5.7. In Figure 5.7 the data has been truncated to 8 bits and displayed as a greyscale image. The most significant 8 bits 
were used. The image has also been cropped and contrast enhanced to allow greater detail to be observed.

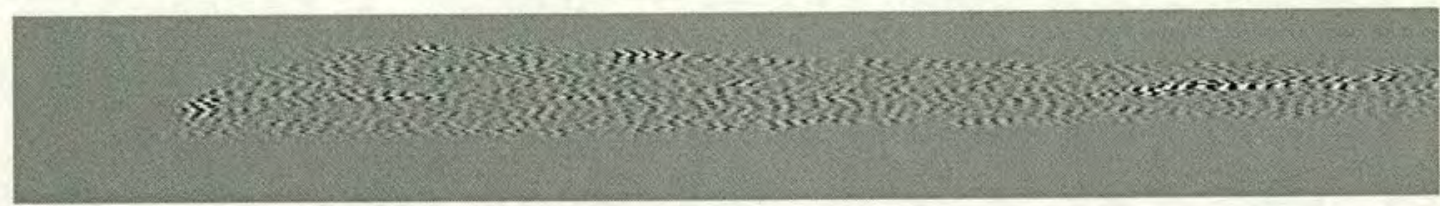

Figure 5.7 Raw phased array data in bitmap format

In each dataset, there were often 37 or 38 separate $2 \mathrm{D}$ images. The Tomtec rotated through a half circle in 36 steps, so the images at the start and end will be repeated (and reversed) images. Therefore the $37^{\text {th }}$ and $38^{\text {th }}$ images were discarded.

Figure 5.8 shows the ultrasound data after being scan converted. This corresponds with the data shown in raw form in Figure 5.7. This comparison of the image given by the Acuson system and the image given by my scan conversion routine shows that my routine gives acceptable results. The surrounding grey area is artificially coloured since that area was not scanned.
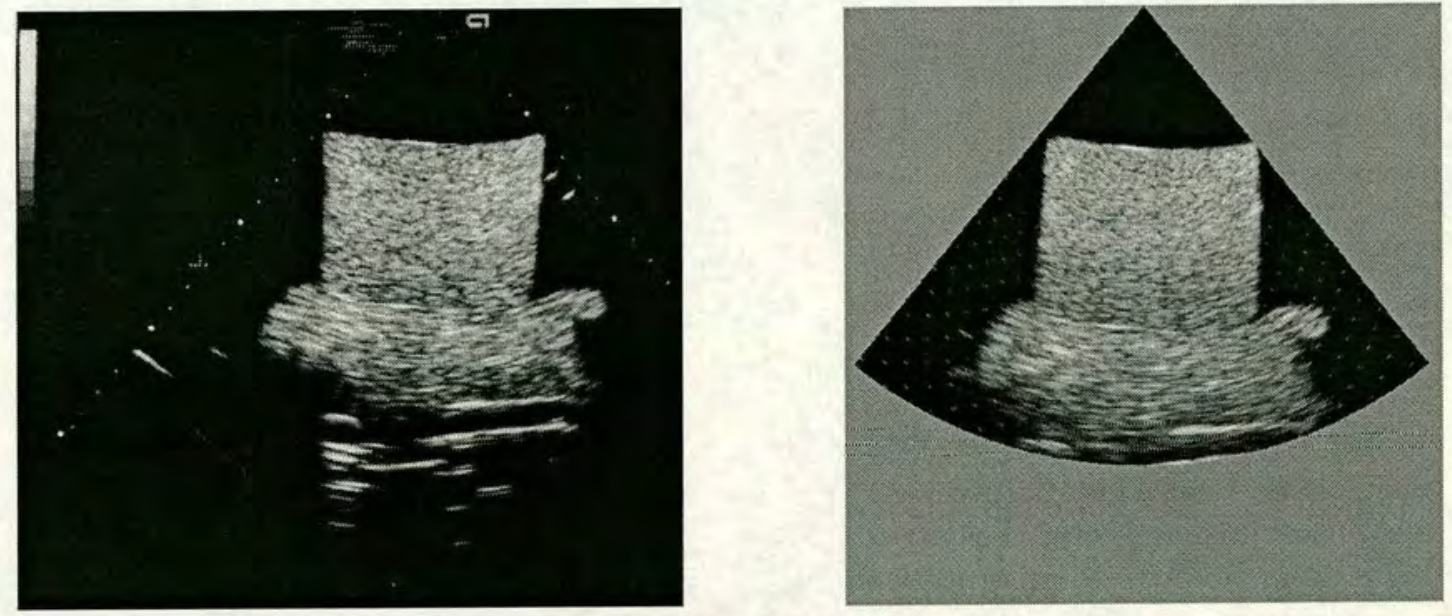

Figure 5.8 Phantom image after being processed for display (left as displayed by Acuson system), (right as reconstructed by myself) 


\section{The Phantom}

The phantom was a (roughly) cubical block of tissue mimicking material. It was approximately $4 \mathrm{~cm}$ cubed. The area immediately below the phantom appears similar in structure (by visual inspection) but is in fact the supporting structure which was several thin kitchen dishcloths. This section of the image is not of interest here.

\subsubsection{Perfect reflector setup}

The near perfect reflector used with the Acuson system was made of steel. It was circular, approximately $46 \mathrm{~mm}$ in diameter and $2 \mathrm{~mm}$ thick. The surface was visibly not completely flat (it had a scratched painted surface). By visual inspection the surface was estimated to be smooth to within approximately one tenth of a millimetre (100 micrometres).

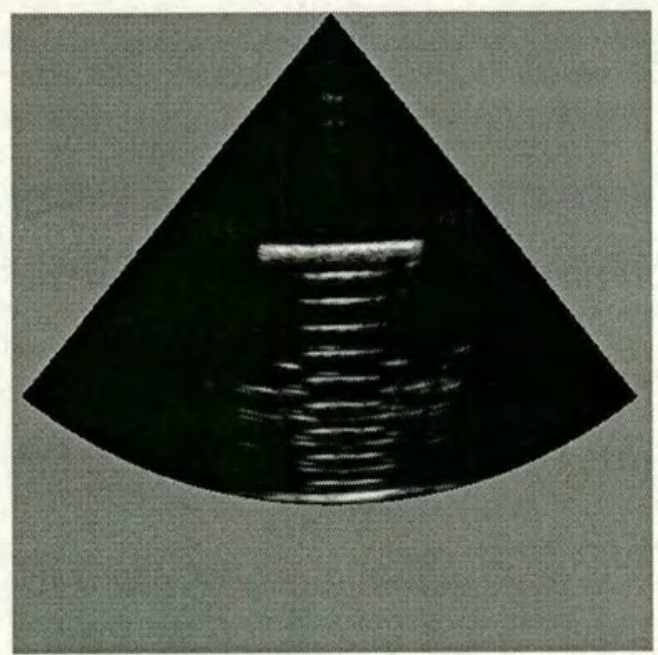

\section{Figure 5.9 Reconstructed image of the perfect reflector used}

The plane reflector did not cover the entire width of the imaged area. (It did cover more than half of the area). The setup for imaging the perfect reflector was identical to the setup for the phantom, with the phantom replaced with the reflector. A reconstructed image of the near perfect reflector is shown in Figure 5.9 which clearly 
shows the width of the perfect reflector. Figure 5.9 also gives some appreciation of the variation in the angle of incidence between the reflector and the ultrasound.

\subsection{Conclusion}

This chapter has described the setups used for each set of data presented in this thesis.

The data and images in this thesis come from only two imaging systems - the single element IVUS system, and the phased array general purpose ultrasound imaging system. In each of these systems, images have been obtained of near perfect plane reflectors, and also of objects that transmit ultrasound (real arteries in vivo and in vitro, and a tissue mimicking phantom).

All of the perfect reflectors used were plane reflectors. Although many researchers use wire reflectors, it is also valid to use a plane reflector, as this gives an accurate representation of the beam characteristics averaged across the whole beamwidth.

The work presented in this thesis was performed on the data in its raw "RF" state, i.e. prior to scan conversion. However, some results are presented after scan conversion for the purpose of interpretation, since this is the standard format for clinical display. 


\section{Chapter 6. \\ RESULTS OF PSEUDO \\ INVERSE FILTERS ON ULTRASOUND \\ NEAR PERFECT REFLECTIONS}

\subsection{Introduction}

This chapter describes the effects of a sigmoid based pseudo inverse filter on a near perfect reflection of an IVUS system's ultrasound signal. The signal that is received as the scanned image of the perfect reflector is shown in Figure 6.1. The circled area indicates the region of interest.

Figure 6.1 presents the signal as a 2 dimensional B-mode image, in which the reflector is visible for only 15 scan lines due to the transducers rotation. The image has not been geometrically corrected, demodulated or log transformed.

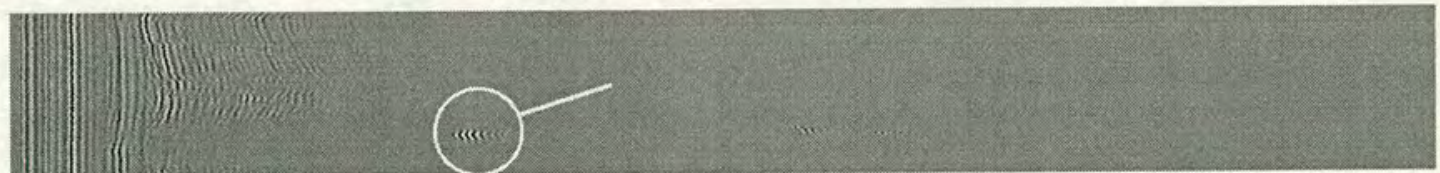

Figure 6.1 The perfect reflector as a $2 D$ image of $R F$ ultrasound intensity

Figure 6.2 shows an A-mode plot (without demodulation) of one particular scan line from Figure 6.1. In this case it happens to be the $186^{\text {th }}$ scanline (counting from the top of the image, with the first line being counted as line zero). There are a total of 240 lines (or rows) in the image, numbered from 0 to 239.

Line 186 was selected because it gave the largest reflected signal from the perfect reflector - after discarding those scanlines where the signal had been clipped due to being too large for the digitiser. 


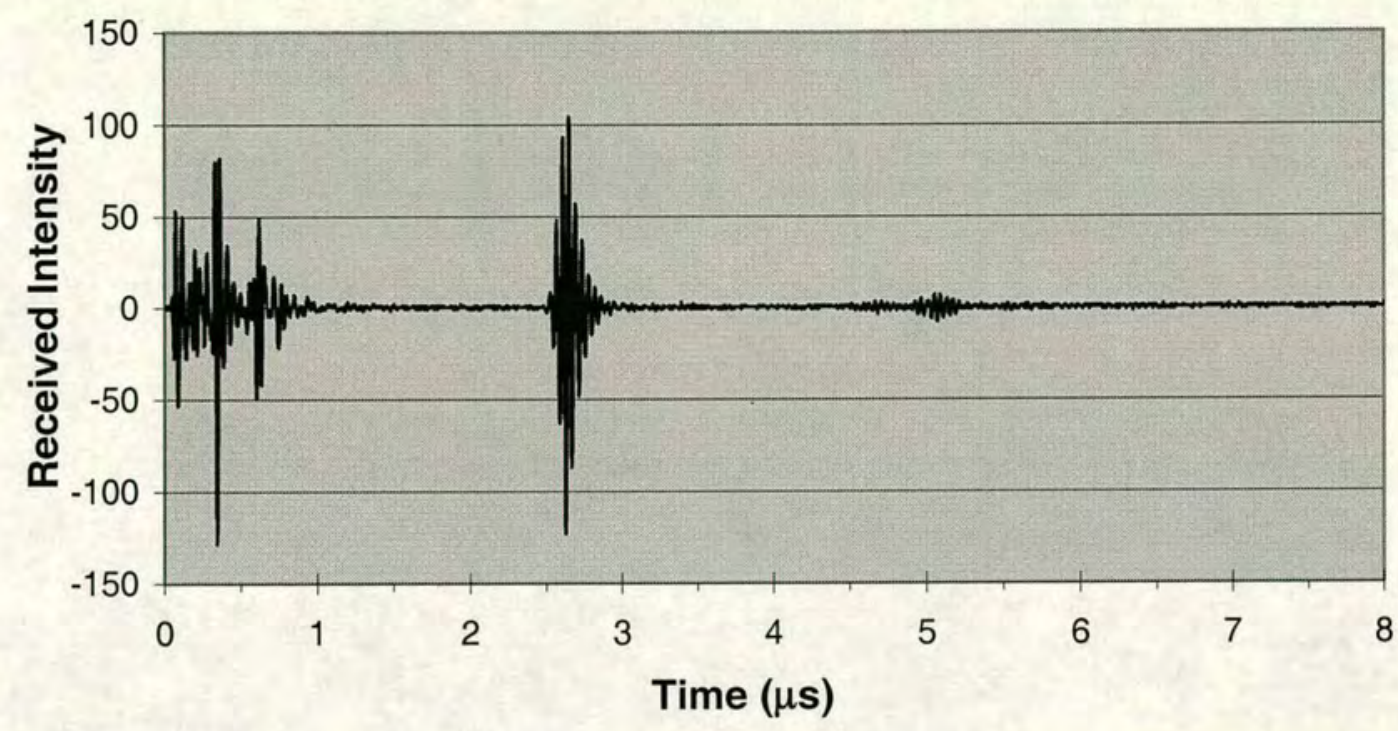

Figure 6.2 The received intensity from a perfect reflector

In Figure 6.2 the left most part of the graph represents the ringdown signal from the transducer, and the near perfect reflection is found between (approximately) $2.6 \mu \mathrm{s}$ and $3.0 \mu \mathrm{s}$. A second (much reduced) perfect reflection can be seen starting at around $5.2 \mu \mathrm{s}$. This signal has probably been reflected by the plane reflector initially, then reflected again by the transducer, and finally reflected a second time by the plane reflector before returning to the transducer to be detected. Thus it is a doubled reflection or a ghost surface.

Figure 6.3 shows the main reflection in Figure 6.2 on an enlarged scale. The region shown corresponds to the range $t=2.4 \mu$ s to $t=3.2 \mu$ s in Figure 6.2.

All results presented in this chapter are the results of applying filters to the signal of Figure 6.2. They will be presented in the same format as Figure 6.3 for easy comparison with the unfiltered case.

It is important to note that the filters were derived using from the same signal that they are being applied to, although the signal was truncated as the first step in deriving the filter. 


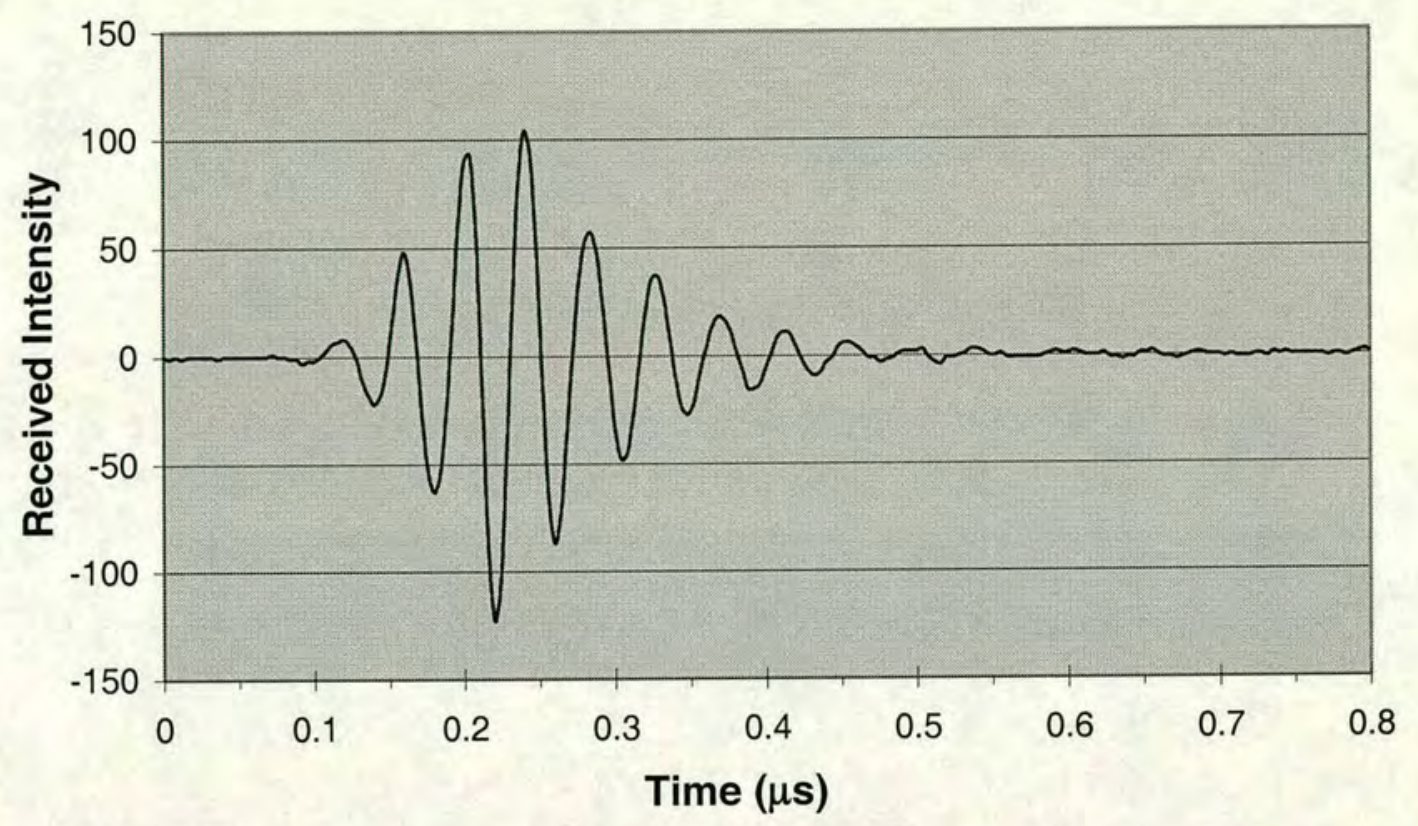

Figure 6.3 The primary pulse received from a perfect reflector

\subsection{Comparison of filtered near perfect reflections with different parameter values}

The graphs presented in this section all represent near perfect reflections that have been filtered with sigmoid based pseudo inverse filters. Different filter parameter values have been used to show the effect that parameter changes have.

The filter results presented here are for the four filters described in Table 4.1. They are presented in order of increasing similarity to the inverse filter. They have the frequency responses shown in Figure 6.4.

In all cases presented in this chapter, the filters use the modified sigmoid based pseudo inverse function given in equation 4.4. Each filter tap sequence has also been Hann windowed, with a length three times as long as the impulse response. 


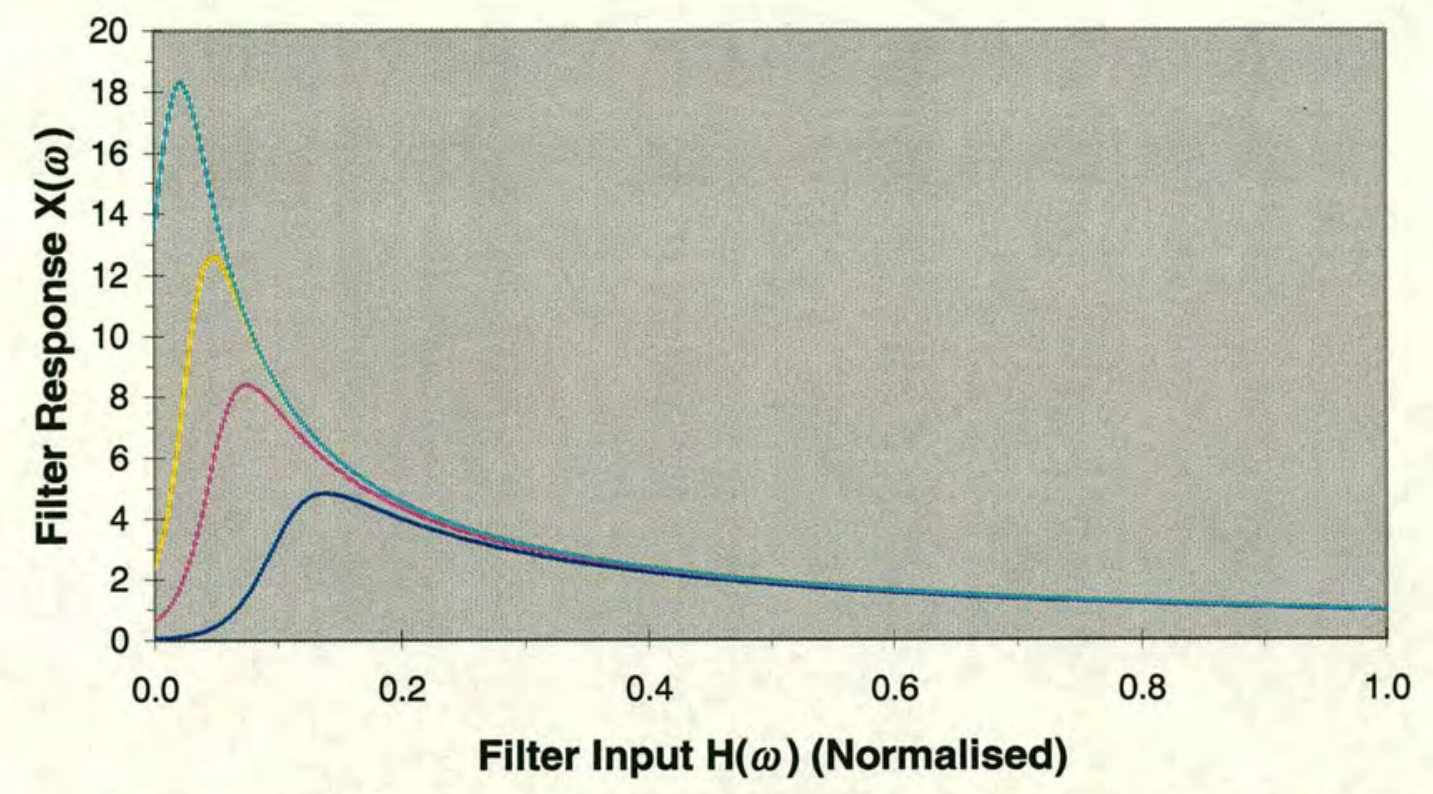

- Sigmoid PI1 $(\mathrm{G}=60 \mathrm{~T}=0.1 \mathrm{~d}=0.05)$
Sigmoid PI3 $(\mathrm{G}=100 \mathrm{~T}=0.03 \mathrm{~d}=0.02)$ Sigmoid PI2 $(\mathrm{G}=80 \mathrm{~T}=0.05 \mathrm{~d}=0.03)$
Sigmoid PI5 $(\mathrm{G}=100 \mathrm{~T}=0.01 \mathrm{~d}=0.02)$

Figure 6.4 The sigmoid based pseudo inverse filter transfer functions presented in this chapter

\subsection{1. "Sigmoid PI1" $(\mathrm{G}=60, \mathrm{~T}=\mathbf{0 . 1}, \mathrm{d}=\mathbf{0 . 0 5})$}

The Sigmoid PI1 filter is the most removed from an inverse filter of the four filters presented in this chapter. The result of applying this filter to the near perfect reflection signal in Figure 6.2 is shown in Figure 6.5.

After the near perfect reflection is filtered with the Sigmoid PI1 filter, the duration of the pulse has reduced from approximately $0.3 \mu$ s to approximately $0.2 \mu$ s. However there is an apparent increase in the level of noise in the surrounding regions. 


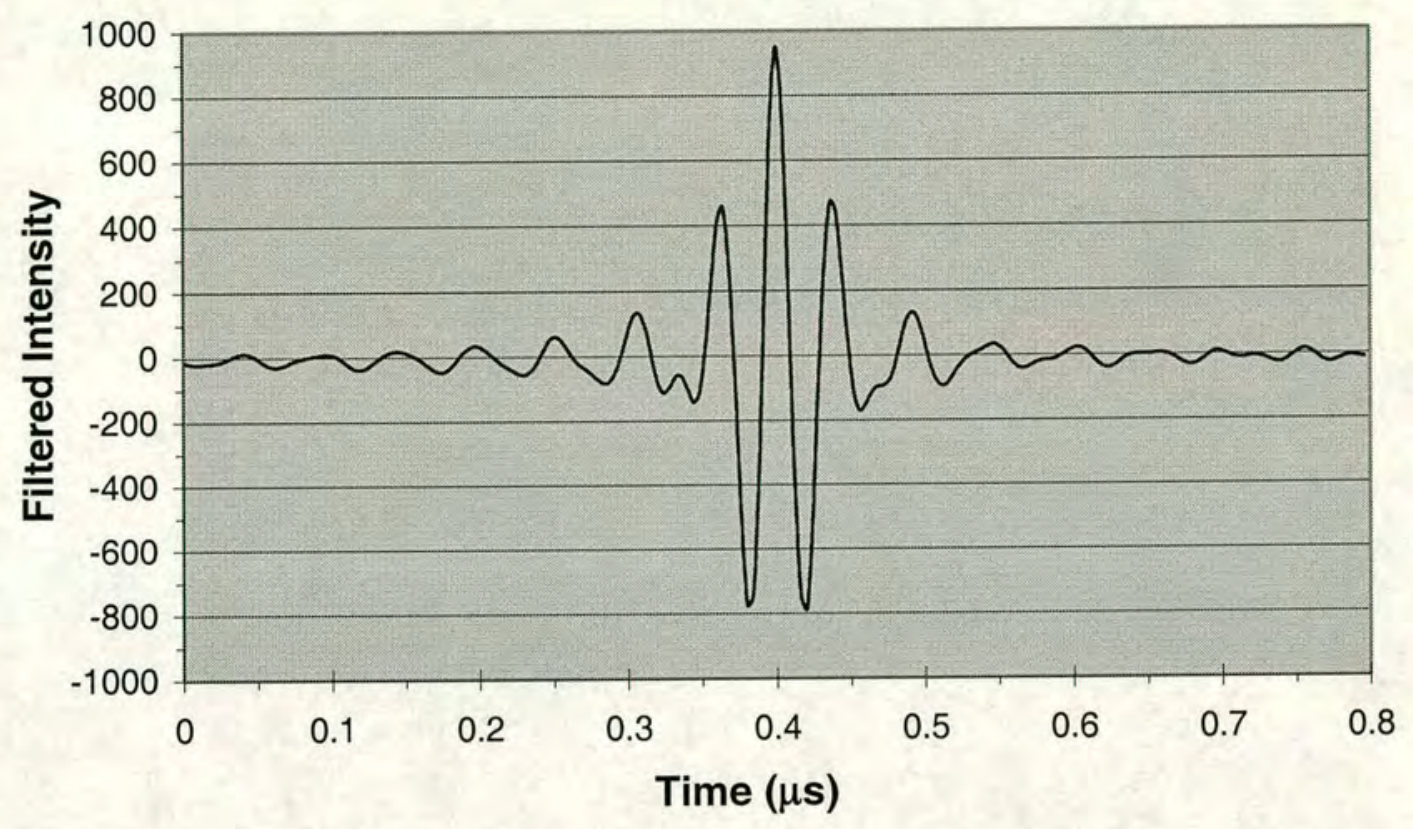

Figure 6.5 Near perfect reflection filtered with modified sigmoid based pseudo inverse filter Sigmoid PII $(G=60, T=0.1, d=0.05)$

\subsection{2. “Sigmoid PI2" $(G=80, T=0.05, d=0.03)$}

The Sigmoid PI2 filter is the second most removed from an inverse filter of the four filters presented in this chapter. The result of applying this filter to the near perfect reflection signal in Figure 6.2 is shown in Figure 6.6.

After the near perfect reflection is filtered with the Sigmoid PI2 filter, the duration of the pulse has reduced from approximately $0.3 \mu$ s to approximately $0.15 \mu \mathrm{s}-\mathrm{a}$ significant reduction. However there is also an apparent small increase in the level of noise in the surrounding regions. The level of noise however does not seem to be greater than the filter with parameters $G=60, T=0.1, d=0.05$, which is somewhat surprising. This may be due to the fact that by allowing a wider bandwidth of signals in the pseudo inversion process, the filter taps may have become closer to a dirac delta function - concentrating more energy around a central point, which results in the filter being less influenced by the applied Hann window function. 


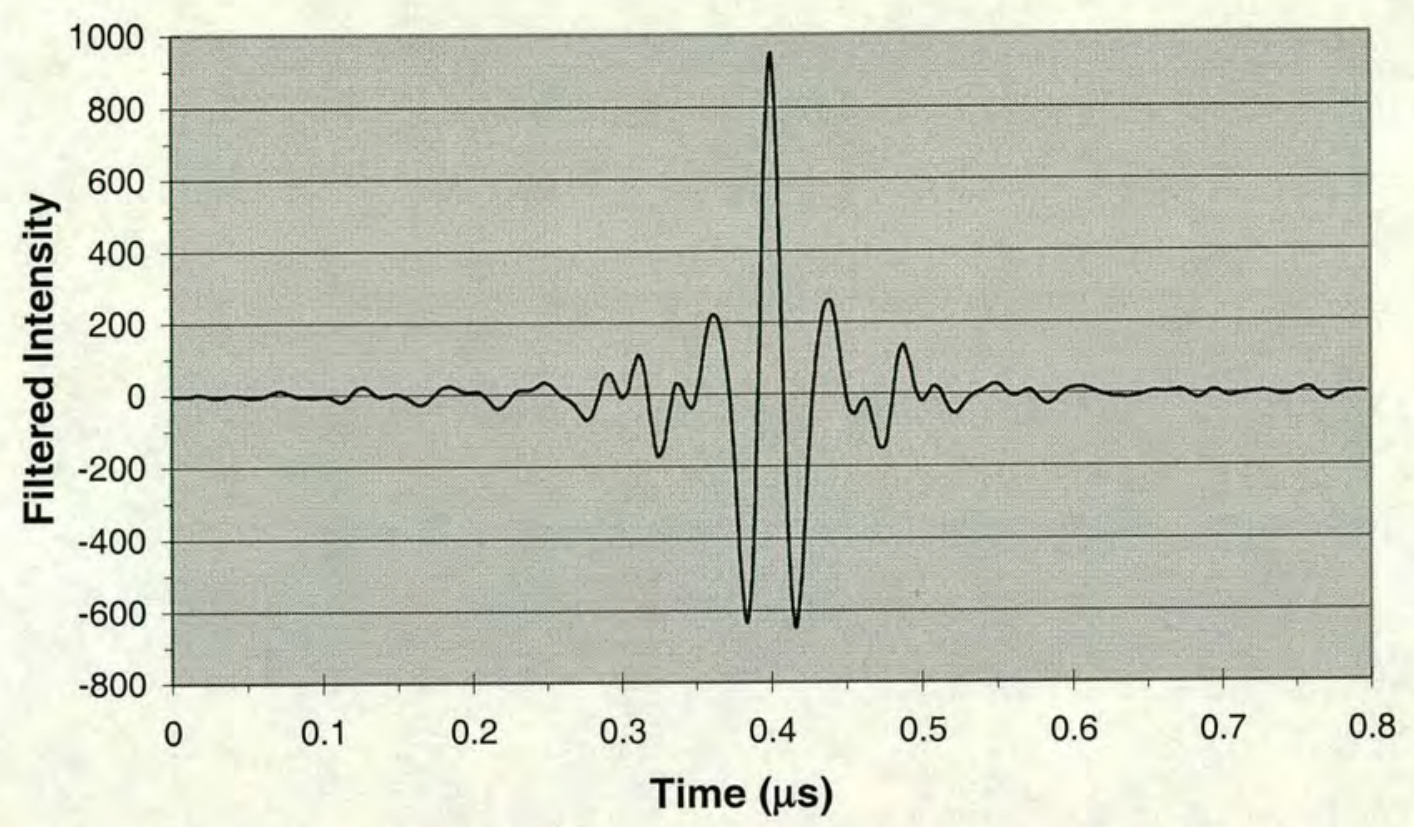

Figure 6.6 Near perfect reflection filtered with modified sigmoid based pseudo inverse filter Sigmoid PI2 ( $G=80, T=0.05, d=0.03)$

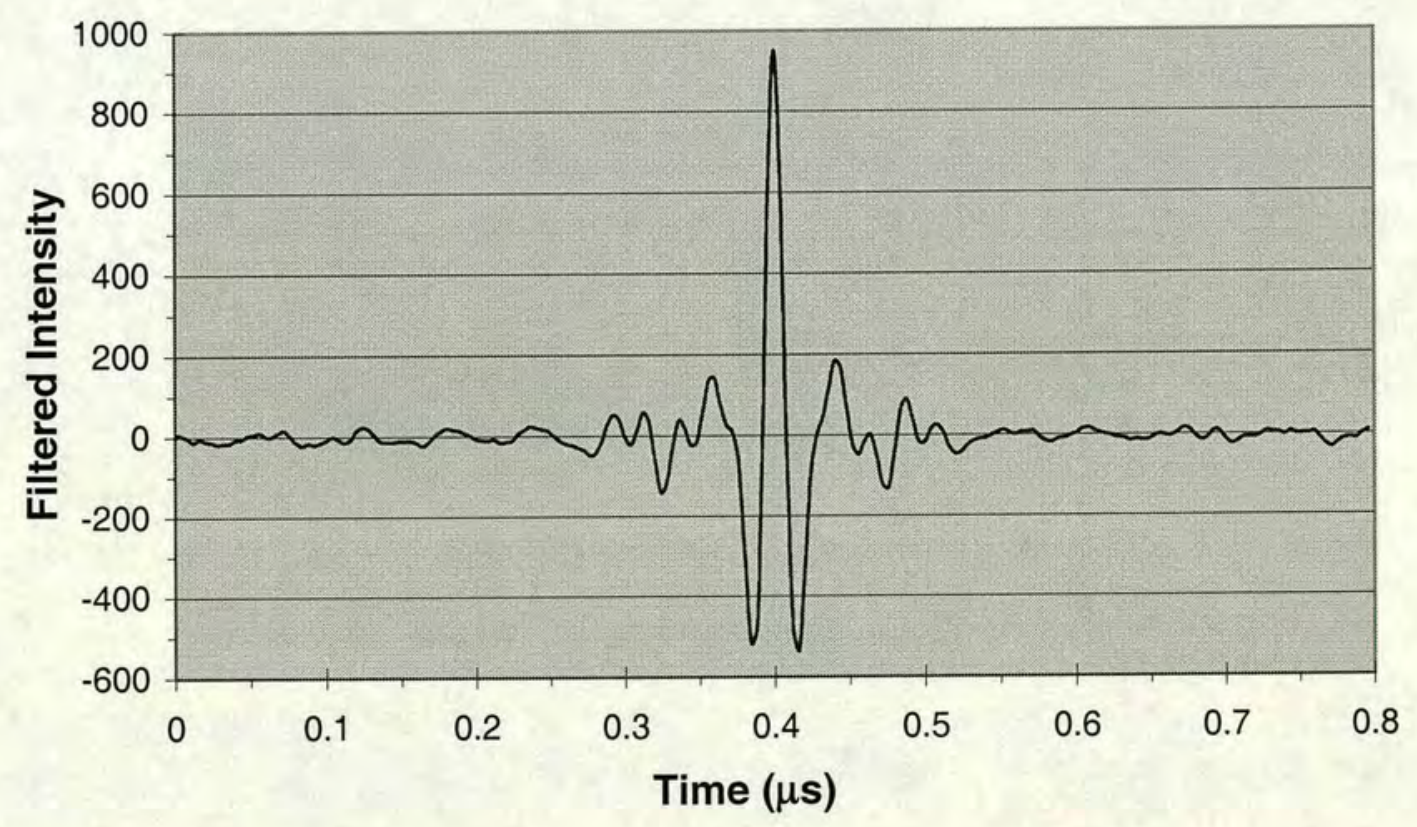

Figure 6.7 Near perfect reflection filtered with modified sigmoid based pseudo inverse filter Sigmoid PI3 $(G=100, T=0.03, d=0.02)$ 


\subsection{3. "Sigmoid PI3" $(G=100, T=0.03, d=0.02)$}

The result of applying this filter to the near perfect reflection signal in Figure 6.2 is shown in Figure 6.7.

After the near perfect reflection is filtered with this filter, the duration of the pulse has reduced from approximately $0.3 \mu$ s to approximately $0.15 \mu \mathrm{s}$ - a significant reduction. However there is also an apparent small increase in the level of noise in the surrounding regions.

When the output signal in Figure 6.7 is compared with the output signal in Figure 6.6 , it can be noted that the magnitude of the side lobes has reduced slightly. However the pulse duration has not reduced significantly.

\subsection{4. "Sigmoid PI5" $(\mathrm{G}=100, \mathrm{~T}=\mathbf{0 . 0 1}, \mathrm{d}=\mathbf{0 . 0 2})$}

The Sigmoid PI5 filter is the closest to an inverse filter of the four sigmoid based pseudo inverse filters presented here. The result of applying this filter to the near perfect reflection signal in Figure 6.2 is shown in Figure 6.8.

After the near perfect reflection is filtered with the Sigmoid PI5 filter, the duration of the pulse has reduced from approximately $0.3 \mu$ s to approximately $0.1 \mu \mathrm{s}-\mathrm{a}$ significant reduction. The size of the side lobes has also reduced significantly. However there is also an apparent small increase in the level of noise in the surrounding regions. In particular the noise seems to contain more high frequency components than was the case with the three previous filters. This is likely to be a problem when applying this filter to medical ultrasound images, as the high frequency noise will be amplified which may degrade the images. 


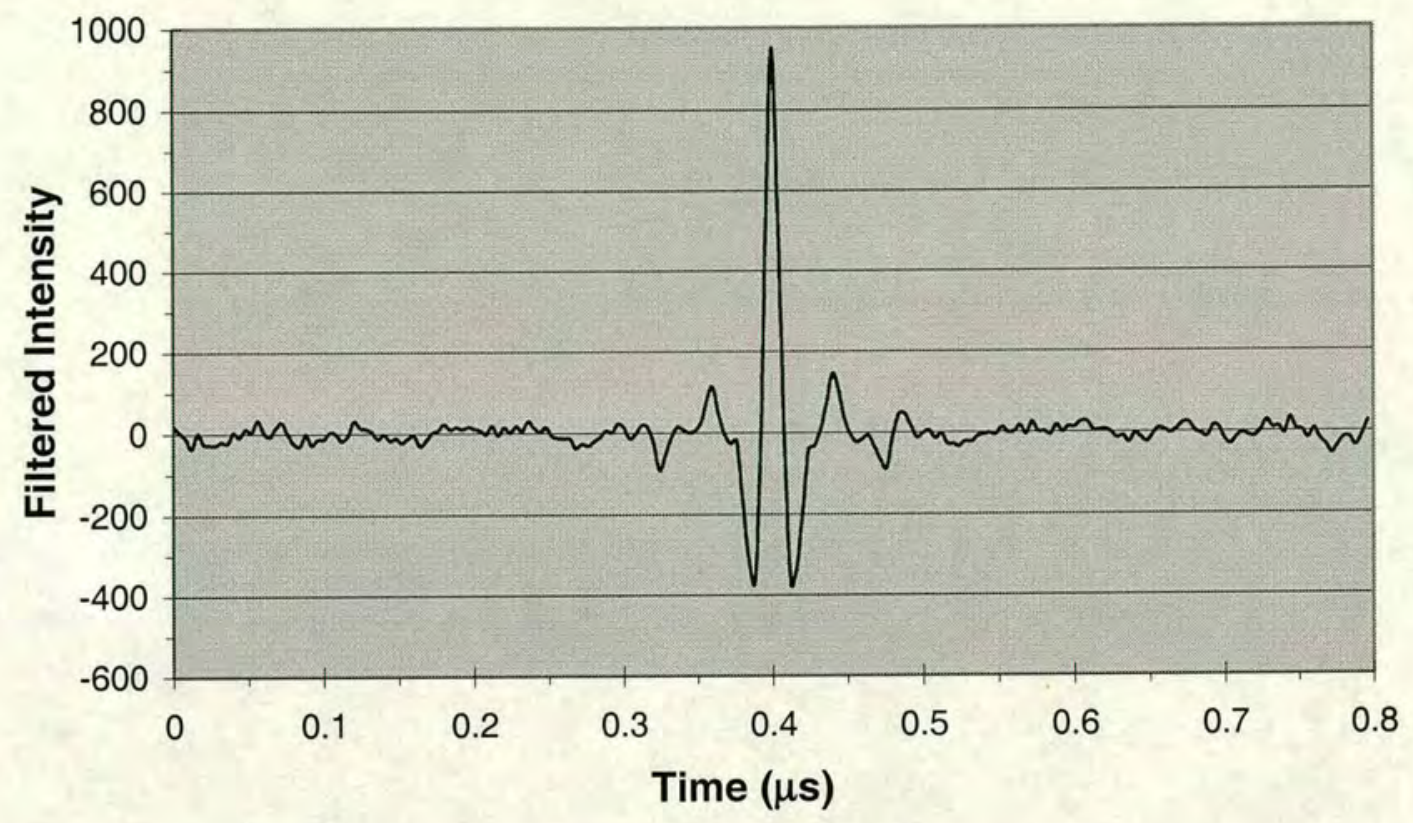

Figure 6.8 Near perfect reflection filtered with modified sigmoid based pseudo inverse filter Sigmoid PI5 ( $G=100, T=0.01, d=0.02)$

\subsection{Comparison with other filters}

It is useful to compare the results of applying sigmoid based pseudo inverse filters to perfect reflections, with the equivalent outputs of other filters such as the matched filter, the inverse filter, the phase correction filter, the square root based filter and Wiener MMSE filters.

Figure 6.9 shows a near perfect reflection after being filtered with a matched filter. This filter is optimal for signal to noise ratio, but clearly lengthens the duration of the pulse. When applied to medical ultrasound images, this spreading effect will result in a blurring of the image, with a reduction in the axial resolution. Therefore this is a poor filter for enhancing the axial resolution of ultrasound imaging systems.

Figure 6.10 shows a near perfect reflection after being filtered with an inverse filter. Clearly the pulse now has reduced in length dramatically. It is important to note that although this result appears to be very good, this is due to the fact that the near perfect reflection being filtered is the same one that the filter was derived from. 
When a different reflection is used, the noise may increase, and the output would appear to degrade significantly.

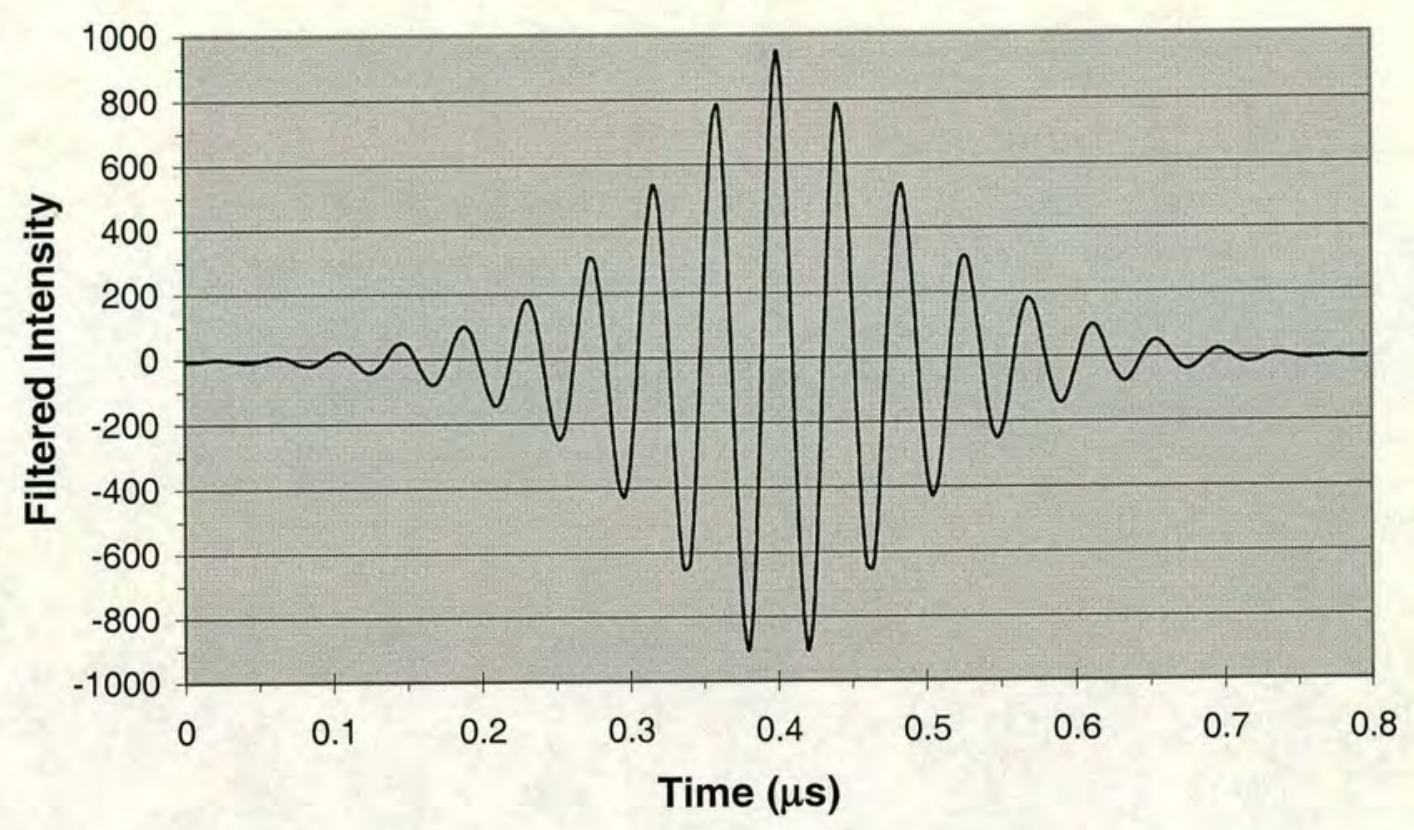

Figure 6.9 Near perfect reflection filtered with a matched filter

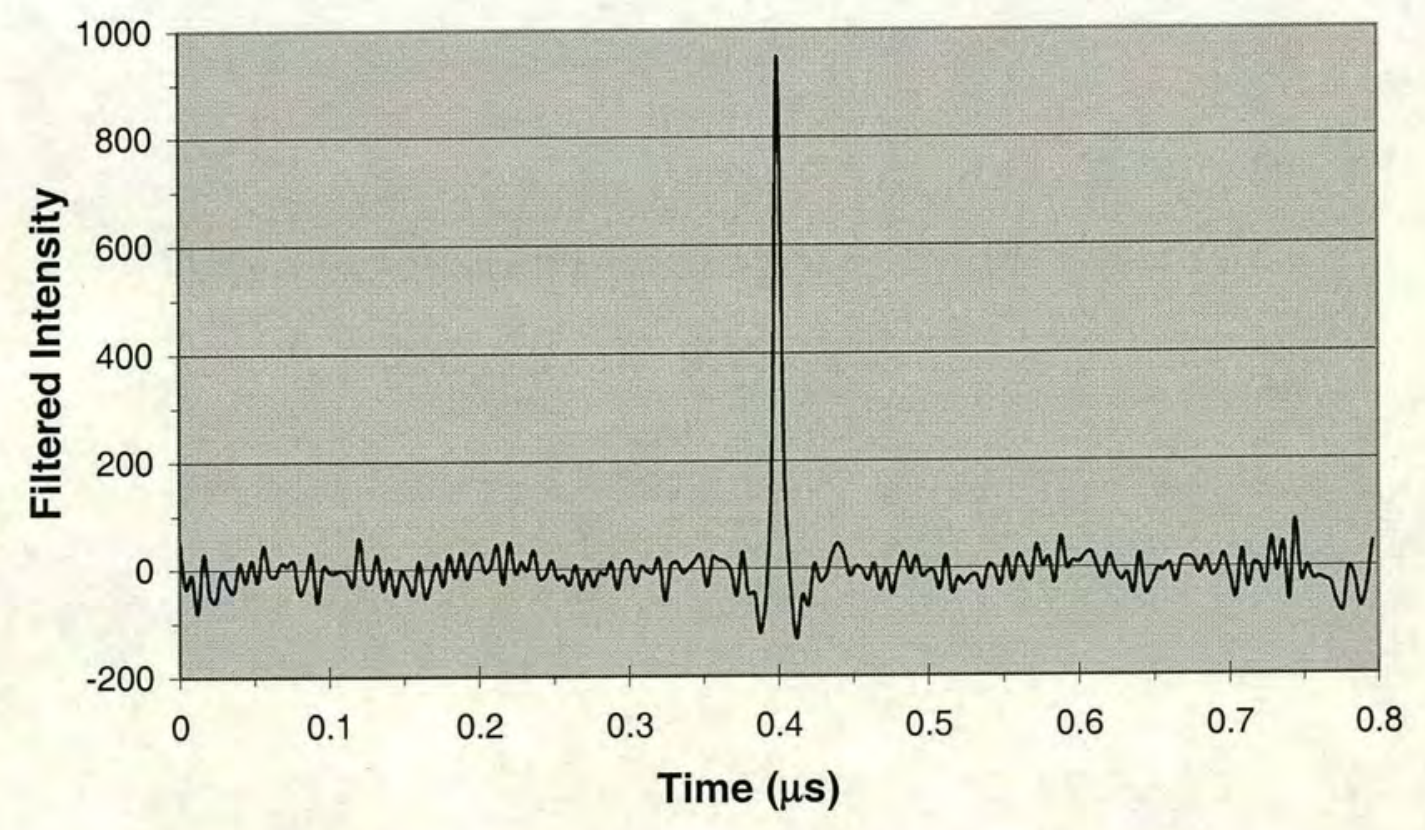

Figure 6.10 Near perfect reflection filtered with an inverse filter 
Note the addition of high frequency components in the noise floor of Figure 6.10. These high frequency components are weak in the signal and amplifying them with the filter is likely to cause high noise levels to result when more complex data is filtered. Therefore this filter is included because it forms a theoretical limit of all pseudo inverse filters - not because it will produce good results in ultrasound imaging.

Figure 6.11 shows a near perfect reflection after being filtered with a phase correction filter. The duration of the pulse has not reduced (c.f. Figure 6.3), although it has changed its shape.

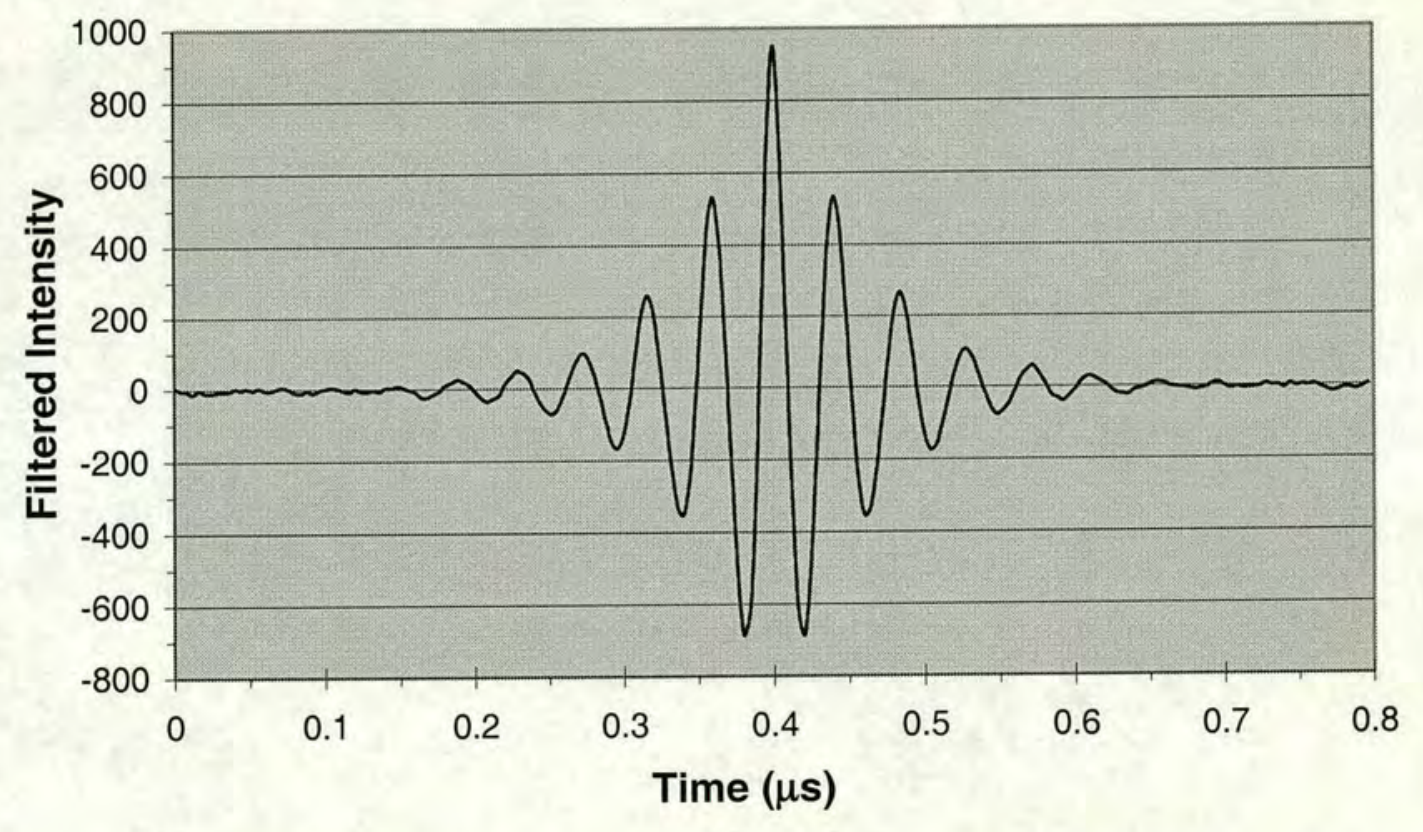

Figure 6.11 Near perfect reflection filtered with a phase correction filter

Figure 6.12 shows a near perfect reflection after being filtered with a square root based filter. The pulse duration has been reduced from approximately $0.3 \mu$ s to approximately $0.2 \mu$ s. Like the inverse filter, there are significant levels of high frequency noise present. 


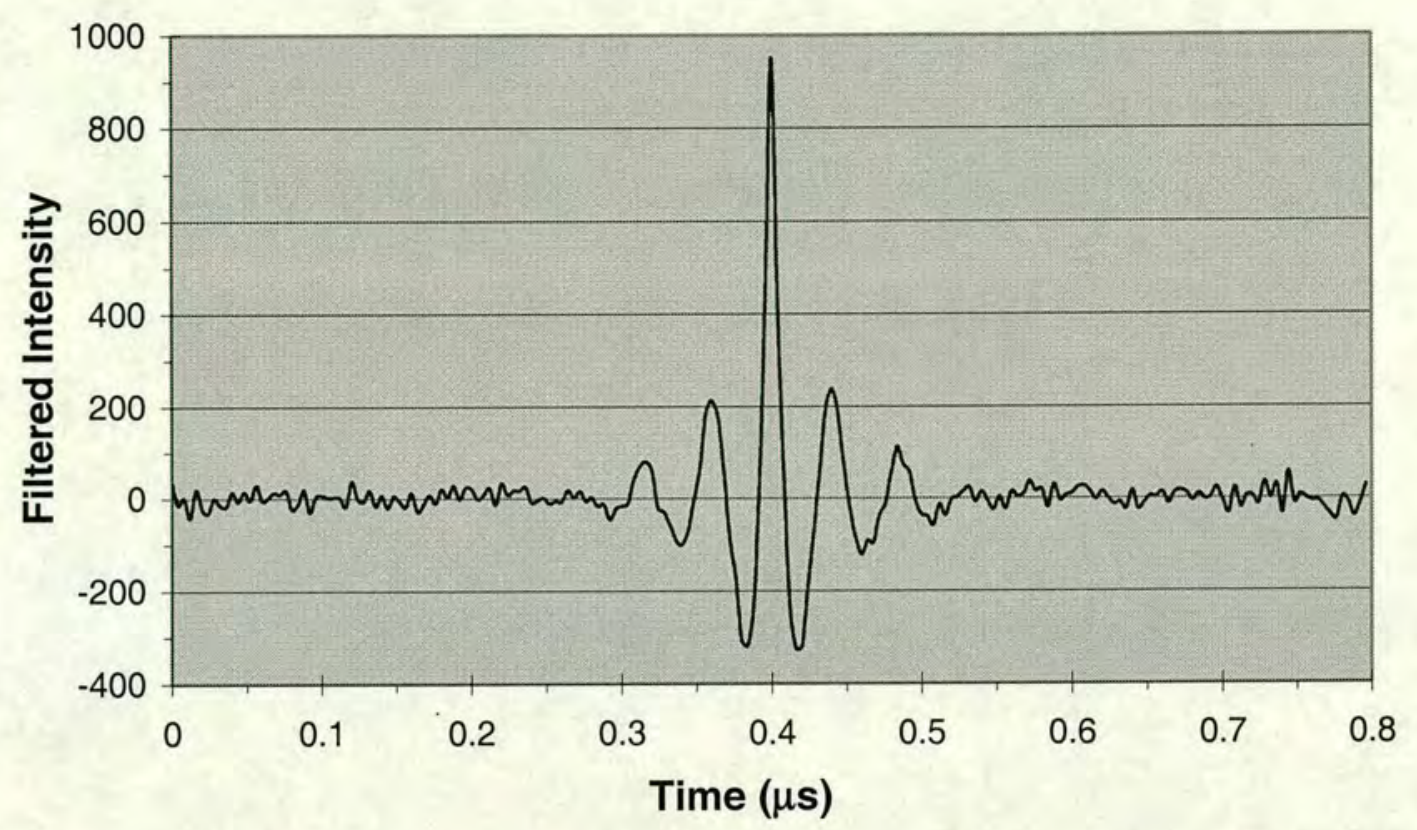

Figure 6.12 Near perfect reflection filtered with a square root based filter

\subsubsection{Comparison with Wiener filters}

Figure 6.13 shows a near perfect reflection after being filtered with a Wiener filter that was designed assuming the noise power to be $100 \%$ of the signal power $(\mathrm{S} / \mathrm{N}=1)$. This makes the filter well removed from an inverse filter; this can be seen in the lack of high frequency noise. The pulse duration has been reduced from approximately $0.3 \mu \mathrm{s}$ to approximately $0.2 \mu \mathrm{s}$. This is a similar result to the Sigmoid PI1 filter.

Figure 6.14 shows a near perfect reflection after being filtered with a Wiener filter that was designed assuming the noise power to be $10 \%$ of the signal power. This makes the filter fairly closely matched with PI3 $(G=100, T=0.03, d=0.02)$. There is not much high frequency noise present, and the pulse duration has been reduced from approximately $0.3 \mu \mathrm{s}$ to approximately $0.1 \mu \mathrm{s}$ - similar to the Sigmoid PI2 and Sigmoid PI3 filters.

Figure 6.15 shows a near perfect reflection after being filtered with a Wiener filter that was designed assuming the noise power to be $1 \%$ of the signal power. This 
makes the filter fairly closely matched with PI5 ( $G=100, T=0.01, d=0.02)$. There is a significant level of high frequency noise present, and the pulse duration has been reduced from approximately $0.3 \mu$ s to approximately $0.1 \mu$ s or less - similar to the Sigmoid PI5 filter.

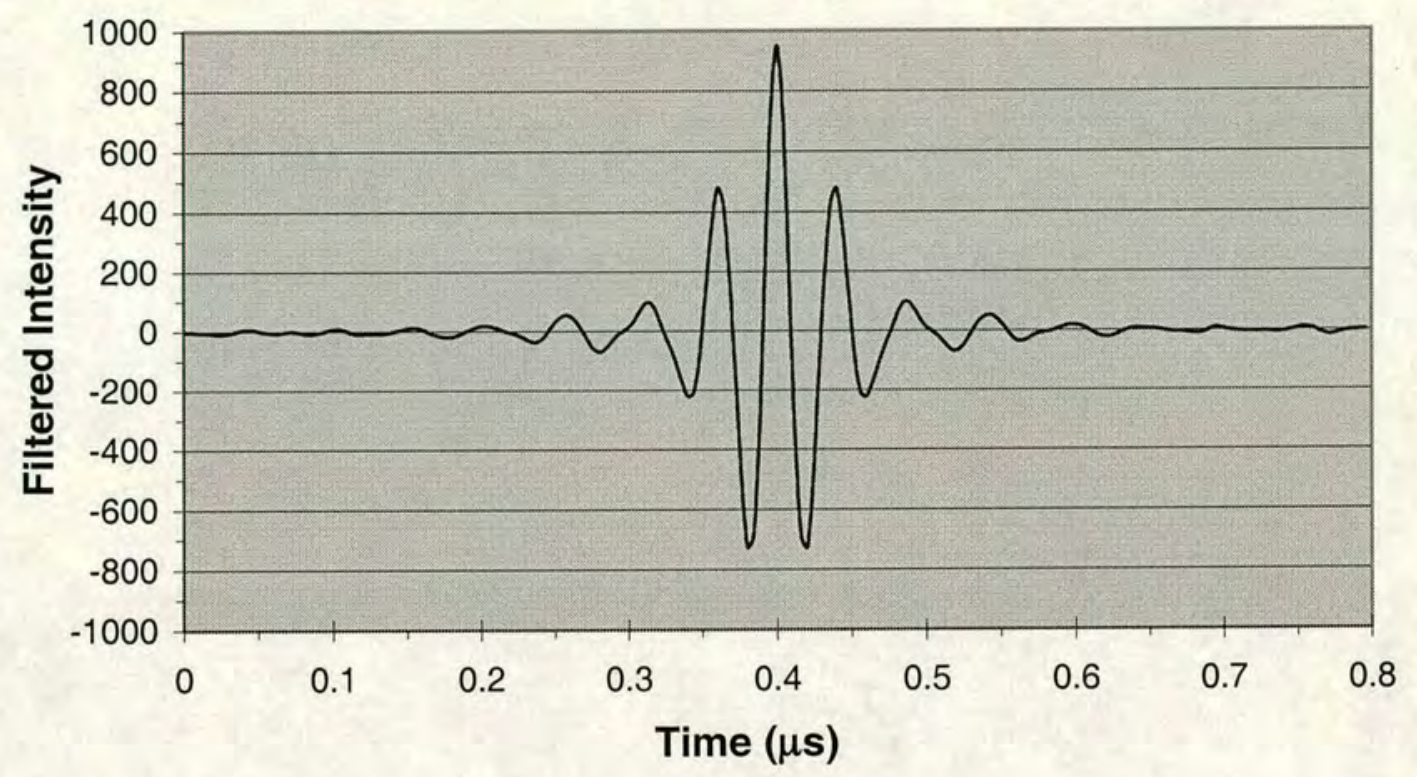

Figure 6.13 Near perfect reflection filtered with a Wiener filter designed for a noise power level of $100 \%$ of the signal power

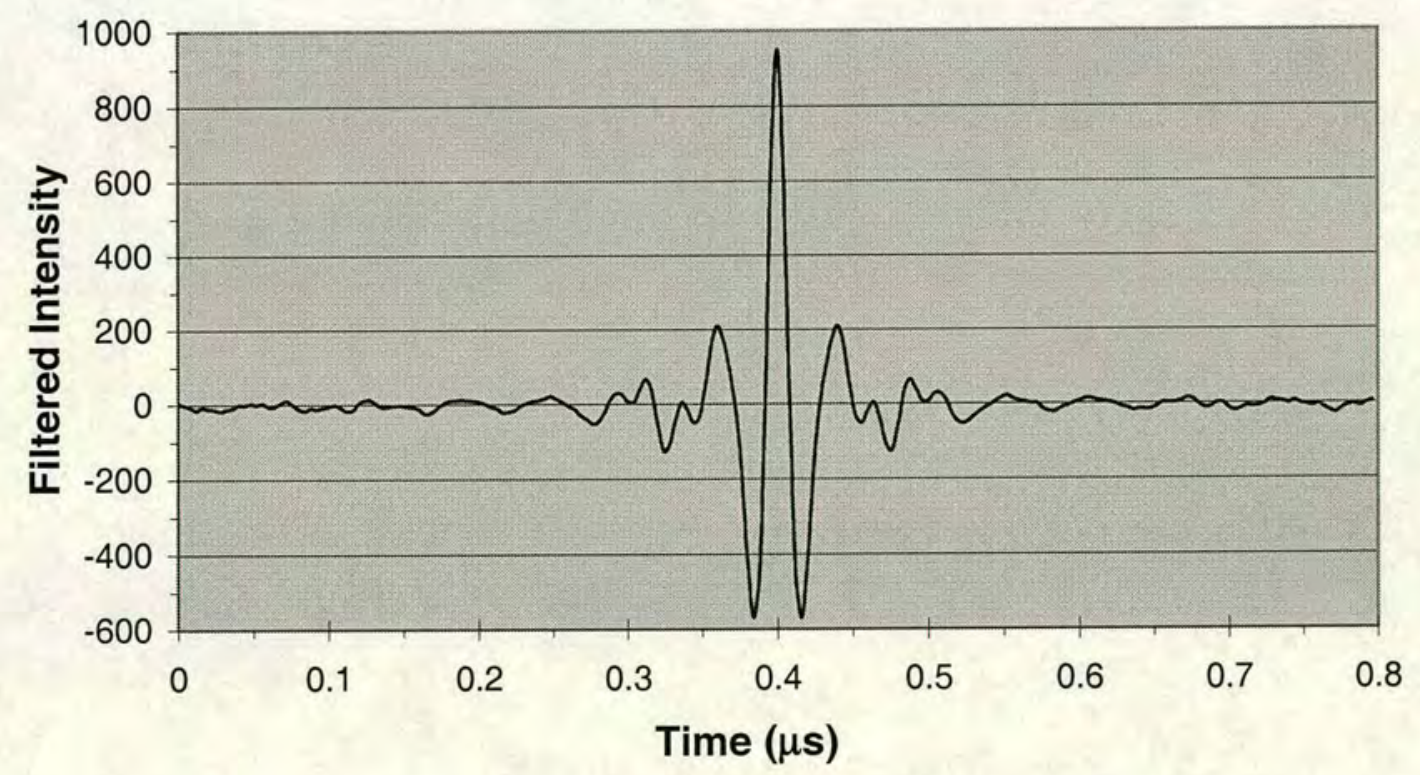

Figure 6.14 Near perfect reflection filtered with a Wiener filter designed for a noise power level of $10 \%$ of the signal power 


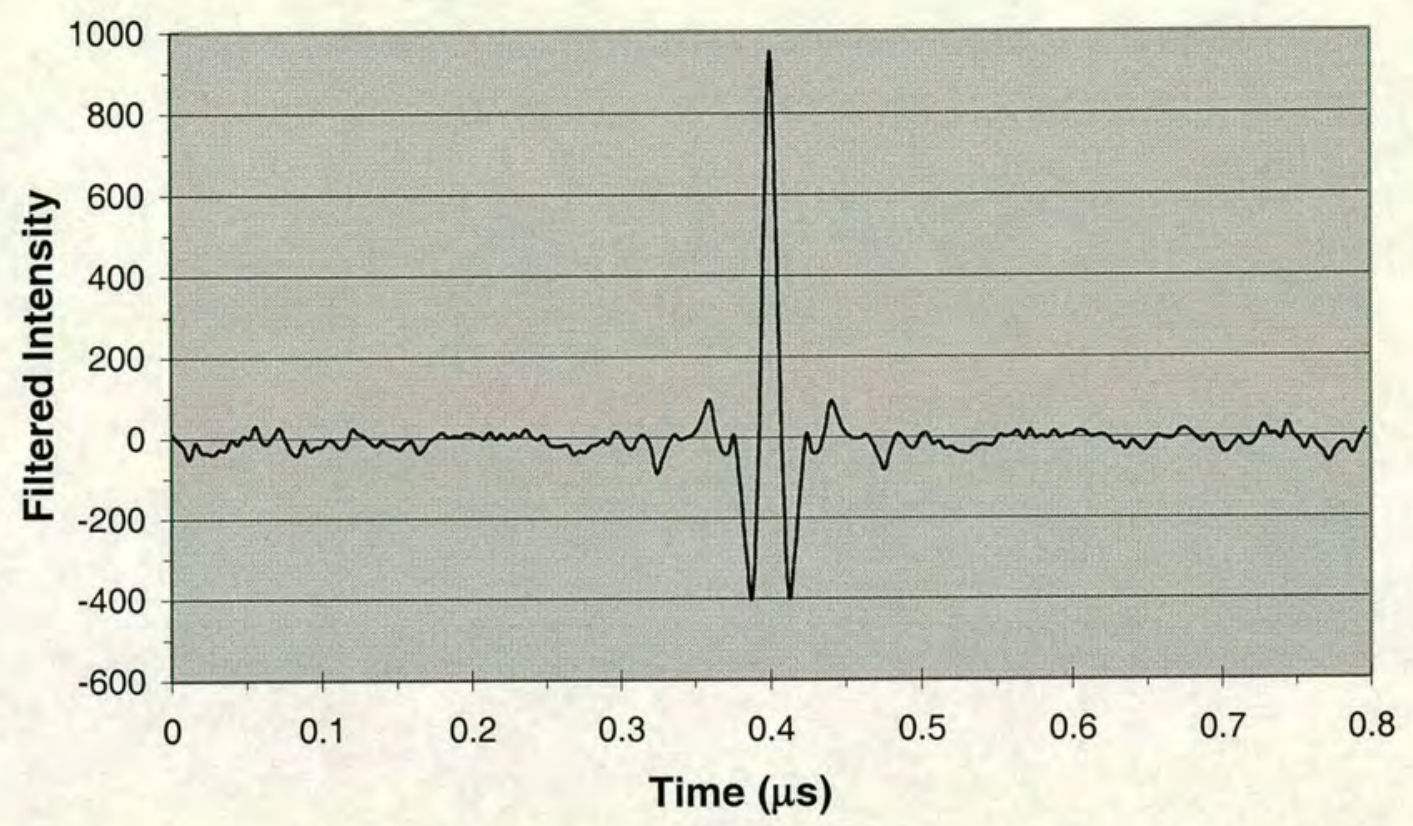

Figure 6.15 Near perfect reflection filtered with a Wiener filter designed for a noise power level of $1 \%$ of the signal power

\subsubsection{Signal to noise ratio analysis}

It is useful to analyse the resulting signal to noise ratio after filtering a near perfect reflection as this will give some indication of one measure of the filters' performance.

It must be noted that this test is somewhat artificial since the signal being filtered is from a near perfect reflector and not from biological objects. This will cause filters that are sensitive to noise (e.g. the inverse filter) to perform better in this test than they will in a biomedical setting. This is because the signal being filtered here is as near to perfect as is possible, and hence should have less noise than will be found in a biomedical image. However this analysis is still worthwhile, despite this limitation, because in a biomedical setting there is a high number of objects that weakly reflect ultrasound; this means that there is no region in a biomedical image where noise alone can be measured without the presence of some residual signal. 
The noise level in each of the filtered near perfect reflections was measured by using the root mean square (rms) value in the regions outside the main oscillations of each waveform (measured from Figure 6.3 and from Figure 6.5 through to Figure 6.15). The signal level was measured as the peak value of each waveform. The resulting signal to noise ratios yielded by each filter are shown in Figure 6.16.

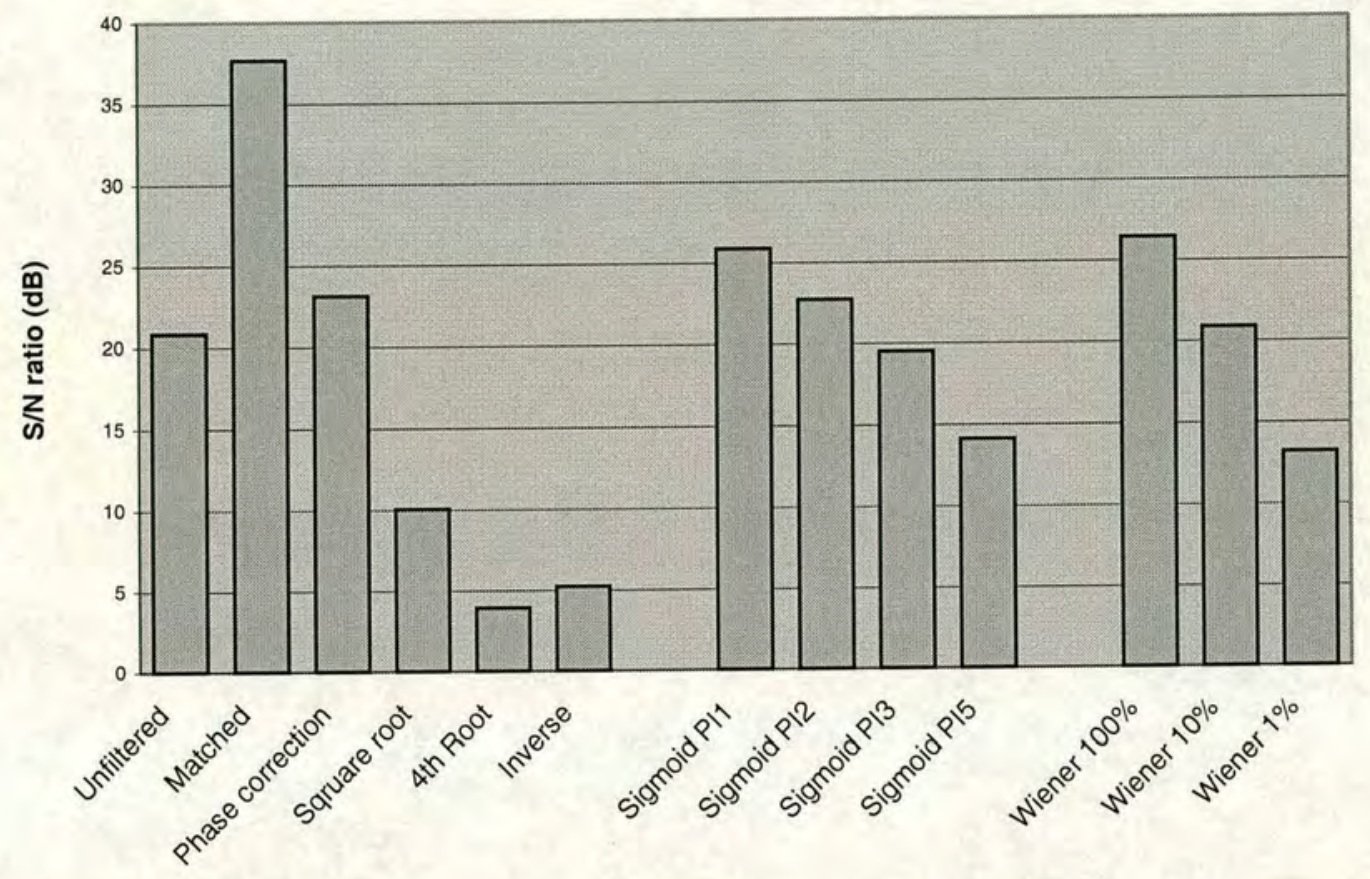

Figure 6.16 Signal to noise ratios for different filters applied to a near perfect reflection

It is clear from Figure 6.16 that the inverse filter and the fourth root filter are the most sensitive to noise, they have yielded they lowest signal to noise ratios. This is consistent with their design procedure - they amplify low level Fourier components more than any of the other filters.

The matched filter has performed better than every other filter in its signal to noise ratio. This is not surprising since the matched filter is theoretically optimal for improving the signal to noise ratio on a stationary signal. It must be recalled that the 
signal to noise ratio is only one component of the filter's performance - the matched filter also degrades the axial resolution of the images.

The Wiener filters have performed well in this test. As the noise level in the Wiener filter design is raised, to bring the Wiener filter closer to the matched filter, the signal to noise ratio that is yielded also approaches that given by the matched filter. Correspondingly, as the noise level is lowered, the resulting signal to noise ratio approaches that of the inverse filter.

The sigmoid based pseudo inverse filters have performed in a similar fashion to the Wiener filters. They have generally performed a trade off between the matched and inverse filters and have yielded a similar signal to noise ratio to the Wiener filters.

\subsection{Conclusion}

Although the near perfect reflection being filtered is also the assumed system impulse from which the filters are derived, it is useful to compare the performance of different filters by filtering the near perfect reflections.

The sigmoid based pseudo inverse filters give results that are comparable with the Wiener filters, and both these are able to trade-off between the theoretical limits of the inverse filter and the matched filter.

The addition of high frequency noise in the inverse filter (and some other filters close to the inverse) indicates that these filters may not give good results when applied to medical images. This is confirmed in the poor signal to noise ratio yielded by the inverse filter - even under the near ideal test conditions of a near perfect reflector. 


\section{Chapter 7.}

\section{RESULTS ON}

PHANTOM IMAGES

\subsection{Introduction}

The results presented in this chapter are from applying sigmoid based pseudo inverse filters to ultrasound images of a tissue mimicking phantom. The transducer used was the phased array system described in Chapter 5 (section 5.3 on page 97). A near perfect plane reflector was also imaged with the same transducer.

Using a phantom will allow analysis of the pseudo inverse filters with a well known structure. The phantom presents a known shape, with a known surface at the top. This allows me to apply the filter and know what the resulting image should (ideally) be. In particular there should be a sharply defined peak in the ultrasound signal, caused by the sharp transition in density between the phantom and the surrounding water. This is useful since in medical images there is a degree of uncertainty about the precise nature and details of the target object in the image. The phantom image presents an opportunity to test the sigmoid based pseudo inverse filter with this uncertainty removed.

\subsection{Application of P.I. filters to phased arrays}

When applying pseudo inverse filters to ultrasound data from a phased array transducer it is important to note that each scanline must be treated separately. That is, the user must design and use a different filter for each scanline (although the same algorithm can be used). 


\subsubsection{Phased array transducer characteristics}

The filters presented here are designed using the scanlines of a perfect reflector image as a measure of the system impulse response for each scanline. The scanlines of the perfect reflector image are different in three ways:

- the angle between the scanline and the reflector varies between successive scanlines (see Figure 7.1);

- the distance to the reflector is different for different lines $($ distance $=d / \operatorname{Cos}(\theta))$ where $d$ is the distance from the transducer to the reflector measured normal (or perpendicular) to the reflector, and $\theta$ is the angle of the scanline from the normal (see Figure 7.1);

- the beamforming is different for each scanline - both on transmit and receive.
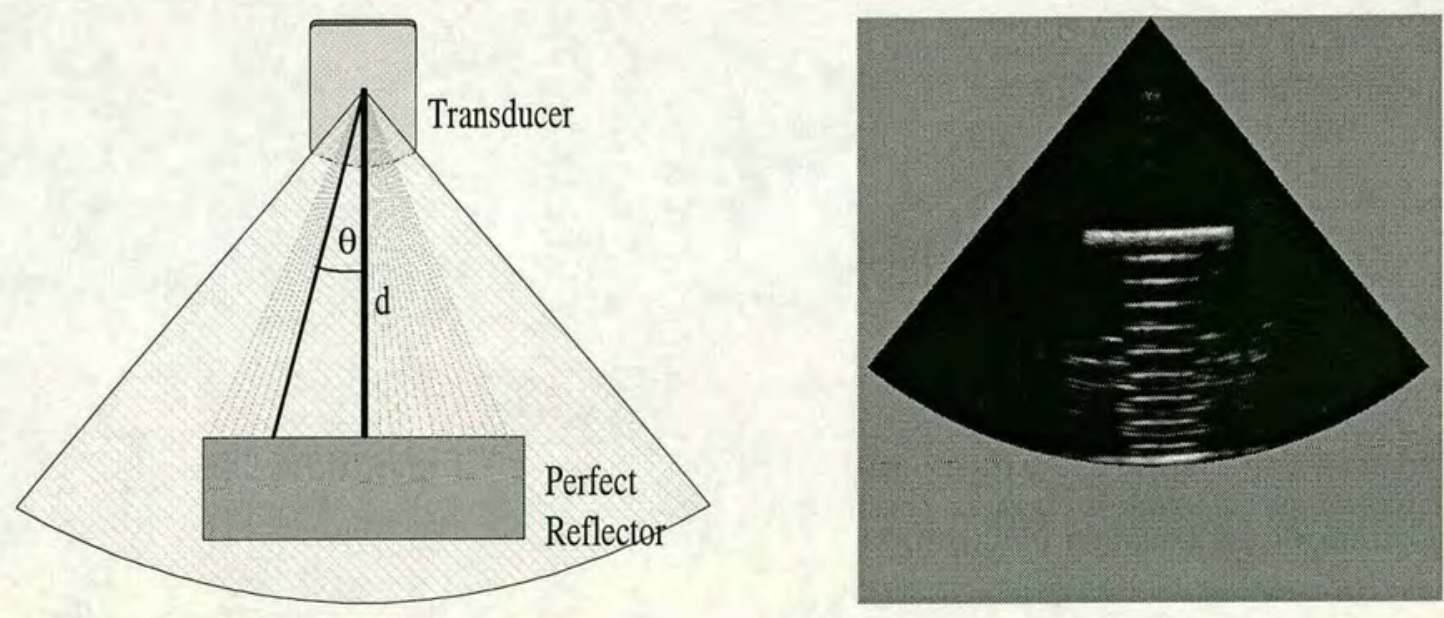

Figure 7.1 The angular and distance variation in the perfect reflector image - the setup (left) and the reconstructed image (right)

The first two differences are due entirely to the geometry of the scanning setup. The distance anomaly was removed by time aligning the scanlines using a cross-correlation. This does not affect the underlying theory or practice of pseudo inverse filter design or application. The amount that each scanline is shifted to remove the distance anomaly is determined by cross-correlating each scanline (in 
turn) with the central scanline (line 65). The location of the maximum value within the cross-correlation vector provides a good estimate of the magnitude to the distance difference between the cross-correlated scanlines. This technique works well with perfect reflector images studied here to within (at worst) a few digitised samples.

The angle anomaly has been ignored at this stage, although this is possibly a small source of error and may warrant further investigation at some future time. It is reasonable to ignore this error because the variations in angle are the same (to a first approximation) for the perfect reflector and the phantom. Also, the changes in angle have not significantly reduced the magnitude of the perfect reflections.
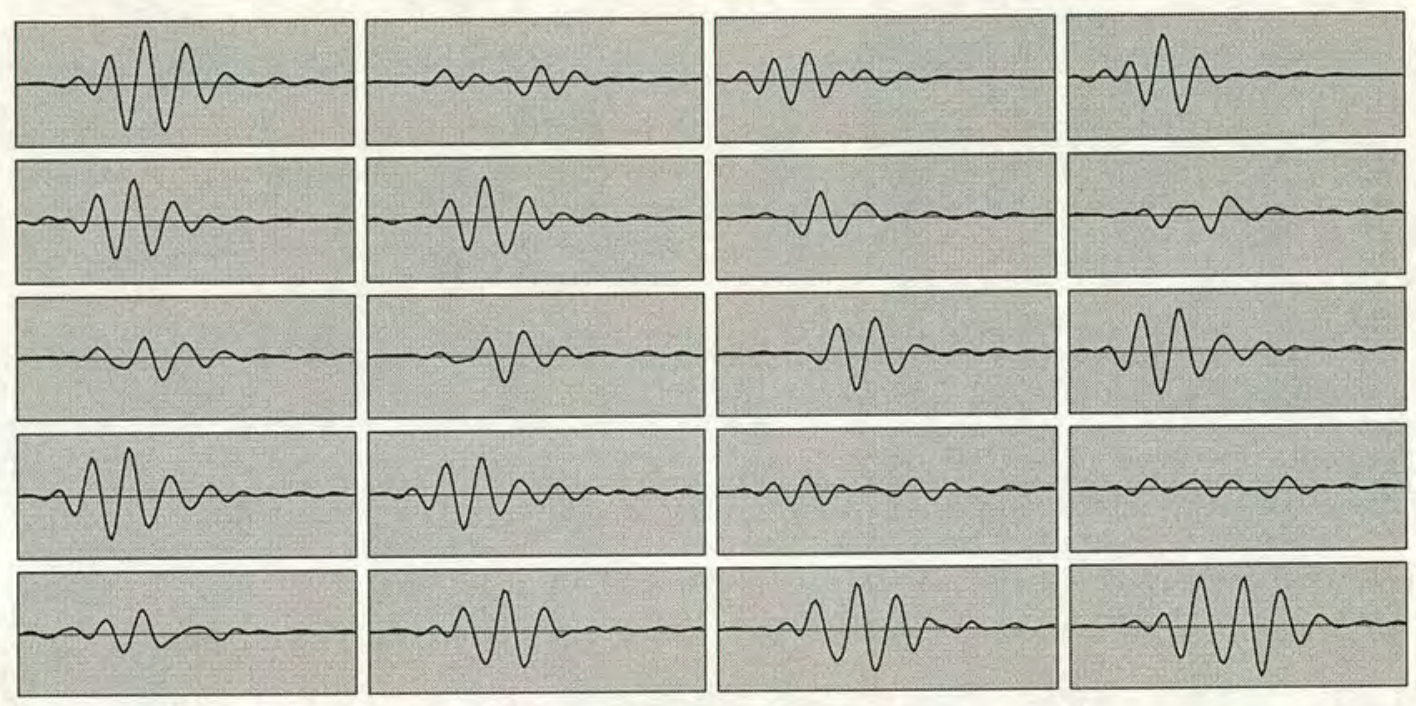

Figure 7.2 Impulse responses of sequential scan lines from a phased array transducer

Figure 7.2 is a sample of what the transducer receives for some different scan lines reflected from a near perfect plane reflector (approximately normal to the central scan line). Reading left to right, then top to bottom, corresponds to adjacent scan lines. The top left graph is the central scan line for the transducer. It can be seen that the characteristics of the received ultrasound signal vary with different scanlines. Although the transducer beamforming patterns cause changes in signal amplitude, it is also clear that some scanlines away from the normal have similar amplitudes to those scanlines at the normal. Therefore it is reasonable to conclude that changing 
the angle of incidence does not significantly attenuate the signal, and that the variations in amplitude are due primarily to the characteristics of the phased array.

The graphs in Figure 7.2 are all on the same (arbitrary) linear scales for both X (time) and Y (received ultrasound intensity) axes. The signals shown are RF ultrasound, the signals have not been demodulated to baseband.

The difference between adjacent scan lines is one source of visual artefacts in medical ultrasound images from phased array transducers. This also creates difficulties in processing the ultrasound signals. To further assess the differences between each scan line, the spectra of the above impulses are shown in Figure 7.3.
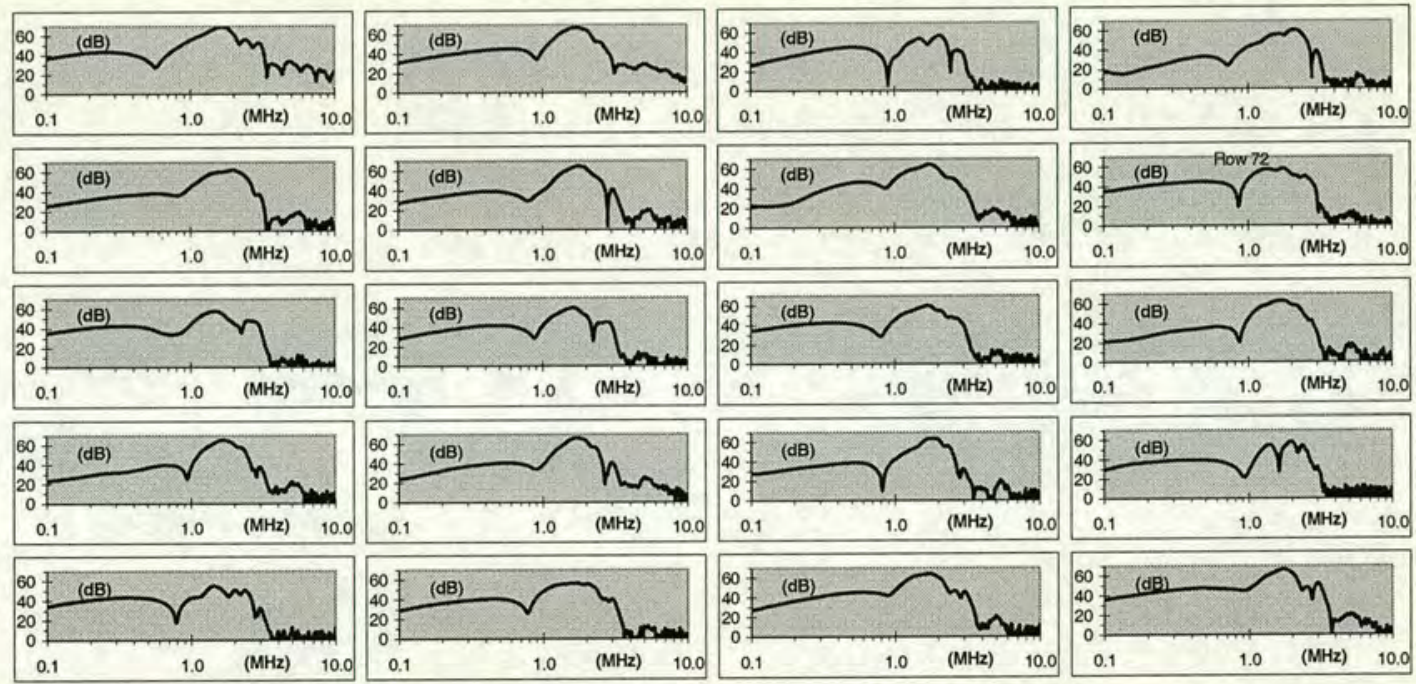

Figure 7.3 Power spectra of the impulse response of sequential scan lines from a phased array transducer

A large range in signal power is evident from the spectra of the various scan-lines (there is over $10 \mathrm{~dB}$ variation in the peak heights). Note also that some spectra contain nulls which present problems to standard inverse filter techniques. The signals do however appear to have a greater similarity in the frequency domain (magnitude) than they do in the time domain in that the overall spectral envelope is the same shape (ignoring the nulls which are caused by destructive interference). 
This suggests that the phase of each Fourier component has a significant variation from line to line.

The graphs in Figure 7.3 are plotted on logarithmic scales, each graph is $70 \mathrm{~dB}$ full scale. Closer inspection (not shown) yields the generalisation that the noise floor in these signals is $40 \mathrm{~dB}( \pm 3 \mathrm{~dB}$ ) below the peak signal level, (where the noise is taken as being the signal level from $4 \mathrm{MHz}$ to $10 \mathrm{MHz}$ ). The noise below $0.8 \mathrm{MHz}$ is substantially higher. The $-40 \mathrm{~dB}$ noise floor is consistent for most scan lines.

\subsubsection{Phased array variance with axial distance}

The observed pulse of ultrasound from a phased array transducer varies significantly with axial distance due to the interference pattern constructed by the many elements in the phased array. Pulses from single element transducers vary less with axial distance. To construct an optimised pseudo inverse filter for any particular axial distance in a phased array system, the designer must measure the system impulse response at that distance. If a designed filter is used at an axial distance different from where it was designed for, then the different system impulse response at that distance will cause the filter's performance to degrade. This leads to a significant difficulty with applying any type of pseudo inverse filter to phased array ultrasound systems - ideally the impulse response needs to be known at every axial distance. This problem is addressed further in section 7.5.

In this chapter, the perfect reflector data (or the system impulse response) is utilised from one distance only (to be precise, one distance for any given scanline, the distance will vary slightly with different scanlines). The phantom to which the filters are applied in this chapter was cubical, and the top surface (closest the transducer) is the principal surface of interest. Unfortunately the distance from the transducer to the top surface of the phantom is not equal to the distance from the transducer to the perfect reflector (compare Figure 5.8 with Figure 5.9). Therefore the results of the filters presented in this chapter are inherently non-optimal. However the results will give a first approximation of the filter's performance. 


\subsection{Image reconstruction procedure}

The images are recorded as two dimensional uncorrected B-mode images. The filters are applied (in the axial dimension only) to the received signal. The image is then scan converted. Scan conversion involves firstly a Hilbert transform for demodulation, secondly a geometric correction using bilinear interpolation, and thirdly a logarithmic transform. The images and signals presented in this chapter are all reconstructed in exactly the same way (except for the different filters that are applied), and are scaled so that the full range of the image represents $50 \mathrm{~dB}$.

The geometric transform was performed so that the reconstructed image covered an angle of $77^{\circ}$. This angle was measured from a sample image printed out by the Acuson 128XP/10 system. This sample image can be seen in Figure 5.8 (left).

The scan converted images are smaller than the uncorrected (received) image, which means the signal is subsampled during the geometric transform - because of the subsampling, the signal is low pass filtered prior to the geometric transform. The LPF in this case is a simple 8 point summation filter (i.e. an 8 point FIR filter with taps $=\{1 / 8,1 / 8,1 / 8,1 / 8,1 / 8,1 / 8,1 / 8,1 / 8\})$.

The validity of this reconstruction procedure is verified in Figure 5.8 which shows that my reconstructed image is similar (exempting the artificial background colour) to the reconstructed image given by the Acuson $128 \mathrm{XP} / 10$ system that was used to obtain the images.

\subsubsection{The example image used}

In this chapter the results of various filters applied to a single example image are presented. The example image was chosen randomly from the available dataset and is shown in Figure 7.4 (the chosen image is frame 9 of 37). The highlighted scanline in Figure 7.4 is the $72^{\text {nd }}$ scanline; this is subject to closer inspection in the following sections. The filters were derived from the perfect reflector data set. It is important to 
remember that the perfect reflector covered only half the beam, so it is not possible (with the data available) to construct separate filters for all scanlines of the image. Only those scanlines close to the centre of the image are able to have filters designed for them (roughly scanlines 30 to 95 out of 131 total scanlines).

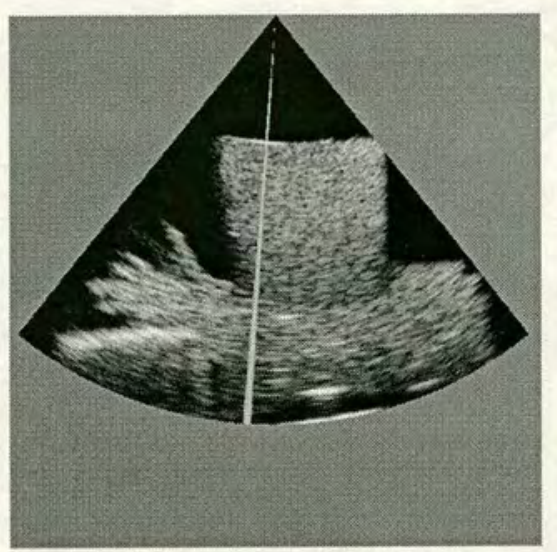

Figure 7.4 The example image with a chosen scanline highlighted (the $72^{\text {nd }}$ scanline)

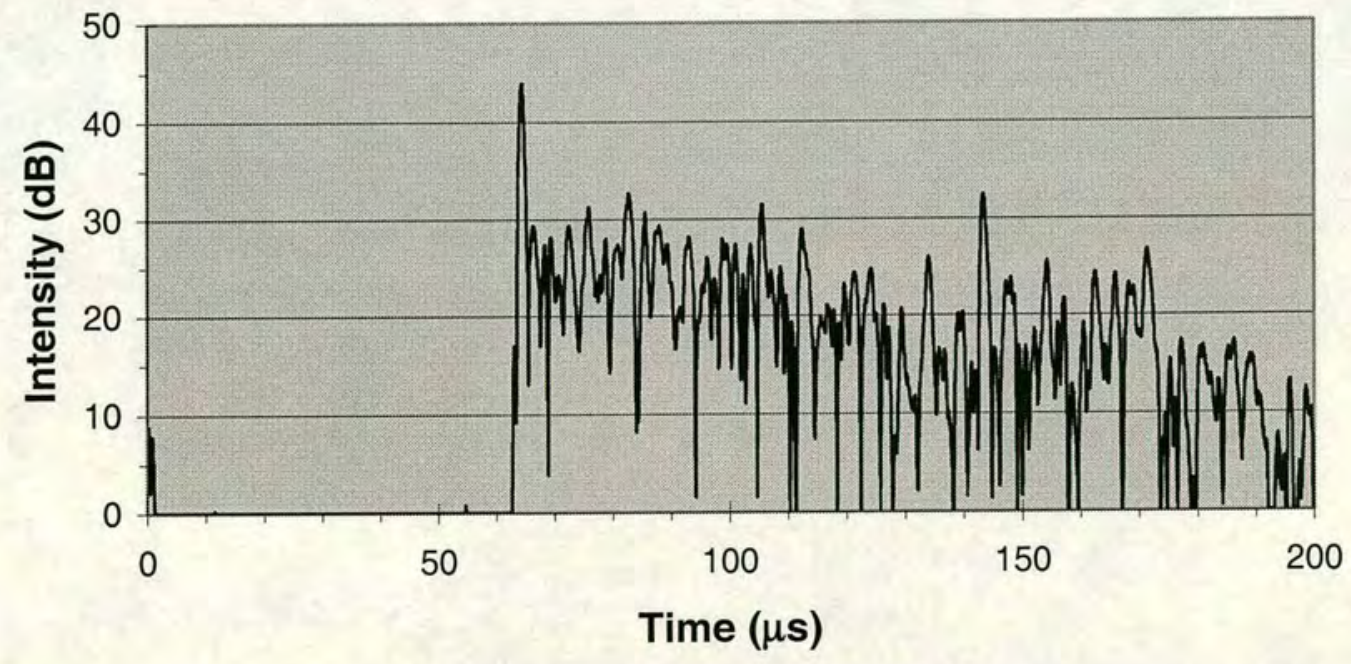

Figure 7.5 The $72^{\text {nd }}$ scanline (after demodulation with a Hilbert transform)

The $72^{\text {nd }}$ scanline is shown in Figure 7.5 after being demodulated with a Hilbert transform. In Figure 7.5 the ultrasound signal reflected by the topmost surface of the phantom is received at $t=64 \mu \mathrm{s}$. This is the principal signal of interest here. The 
bottom surface of the block reflects ultrasound signals that are received at approximately $t=140 \mu \mathrm{s}$. All signals received after $t=140 \mu \mathrm{s}$ are reflected from the material that was supporting the phantom, and are not considered here. The main signal of interest is more clearly visible in Figure 7.6, which is an enlarged version of Figure 7.5

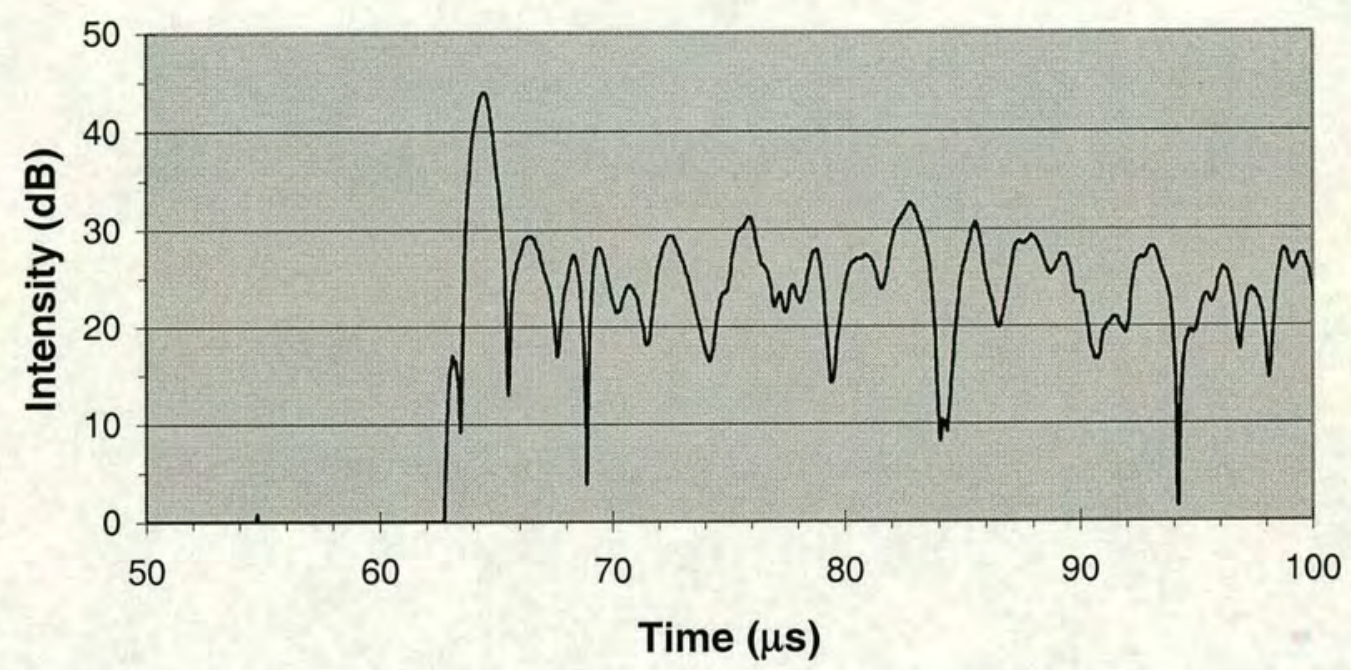

Figure 7.6 The phantom edge in the $72^{\text {nd }}$ scanline (after demodulation with a Hilbert transform)

\subsection{Images filtered with sigmoid based PI filters}

The example image shown in Figure 7.4 was filtered with the four Sigmoid based pseudo inverse filters presented in Table 4.1. The filters were designed using an image of a near perfect plane reflector, imaged with the same transducer. A separate filter was designed for each scanline, and applied only to the scanline for which it was designed. 


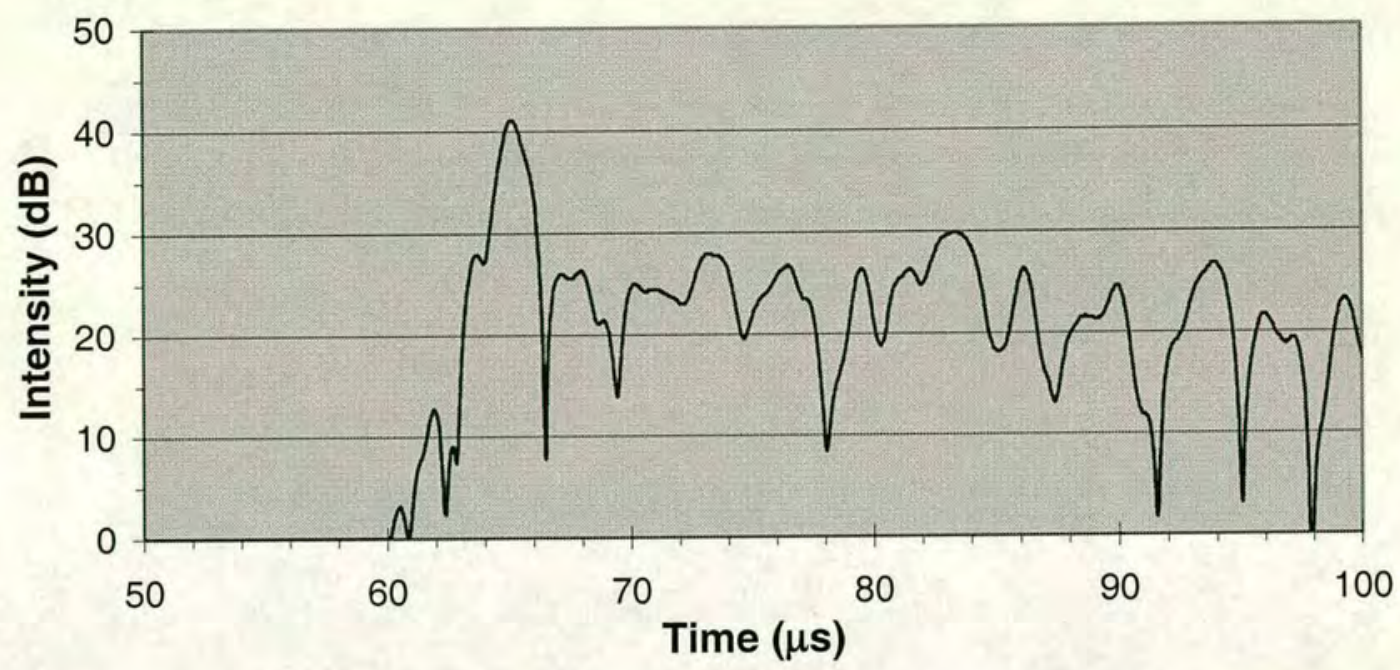

Figure 7.7 The $72^{\text {nd }}$ scanline filtered with Sigmoid PII (after demodulation with a Hilbert transform)

Figure 7.7 shows the $72^{\text {nd }}$ scanline after it was filtered with the filter "Sigmoid PI1", and demodulated with a Hilbert transform. Comparing Figure 7.7 with Figure 7.6, it is clear that the main lobe at $t=64 \mu \mathrm{s}$ is wider than in the unfiltered case when measured 10 to $20 \mathrm{~dB}$ below the peak.

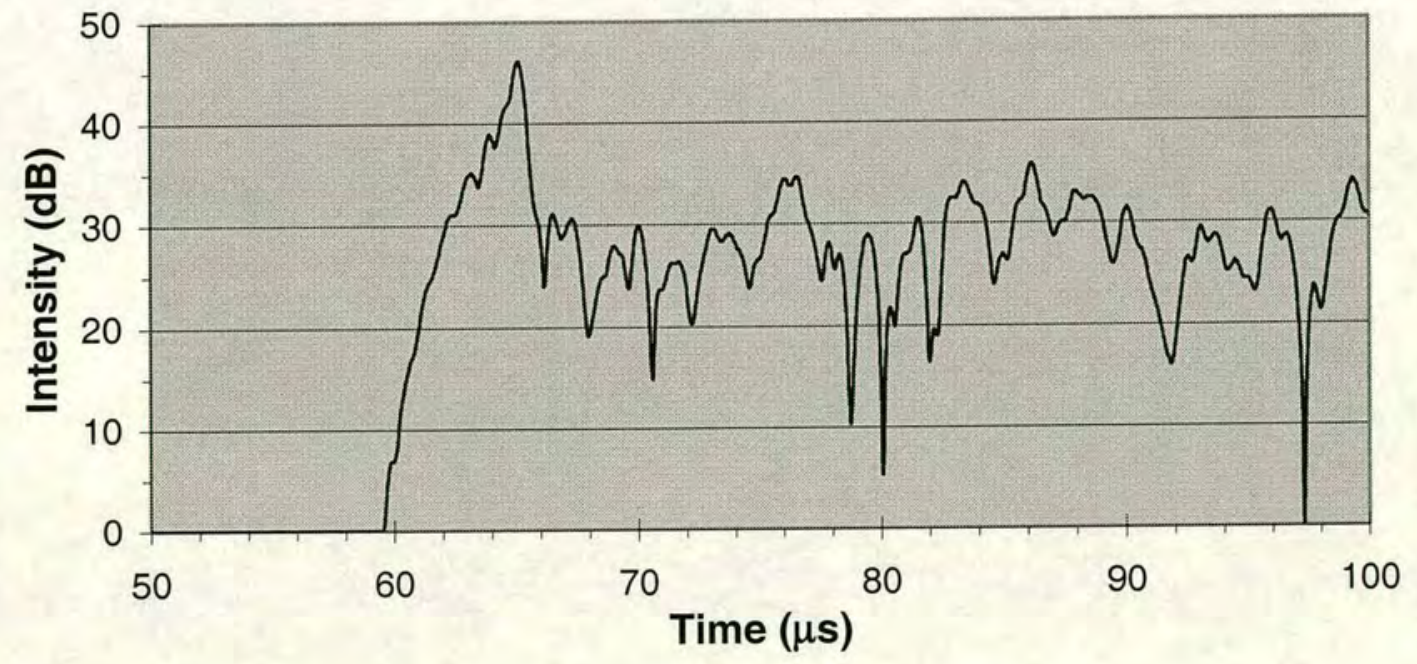

Figure 7.8 The $72^{\text {nd }}$ scanline filtered with Sigmoid PI3 (after demodulation with a Hilbert transform) 
Figure 7.8 shows the $72^{\text {nd }}$ scanline after it was filtered with the filter "Sigmoid PI3", and demodulated with a Hilbert transform. The main lobe at $t=64 \mu \mathrm{s}$ is clearly wider than in the unfiltered case (Figure 7.6) when measured 10 to $20 \mathrm{~dB}$ below the peak, and is wider than after filtering with the filter Sigmoid PI1. However the lobe is narrower when measured $3 \mathrm{~dB}$ below the peak.

In this case the main lobe width has increased (especially on the left hand side) so much that other image details at the location corresponding to $60 \mu$ s on Figure 7.8 could be obscured. This is a significant reduction in image resolution.

The narrowing of the main lobe at the peak would help lead to more accurate detection of the edge location by automated edge extraction algorithms, so this aspect is a good result for this filter.

\subsubsection{Analysis of main lobe width vs. side lobe suppression}

It is useful to observe how the proposed sigmoid based pseudo inverse filters perform with respect to the width of the main lobe. This has been compared with the relative height of the side lobe to right of the main lobe. The width of the main lobe is estimated using linear interpolation between discrete sample points (the points are separated by $50 \mathrm{~ns}$ or $0.05 \mu \mathrm{s}$ ). It has been measured as the full width at half power (i.e. measured $3 \mathrm{~dB}$ below the peak). The measurements are presented in Table 7.1 and are plotted in Figure 7.9.

Although the main lobe width has increased at 10 to $20 \mathrm{~dB}$ below the peak, it has decreased when measured $3 \mathrm{~dB}$ below the peak for the filters Sigmoid PI3 and Sigmoid PI5 (and marginally for Sigmoid PI2). The relative height of the first side lobe to the right of the main lobe has remained approximately the same. Although this result shows some degree of improvement in narrowing the main lobe, the extra lobe width at 10 to $20 \mathrm{~dB}$ below the peak could obscure other image details and thereby reduce the image resolution. This problem is addressed in section 7.5. 


\begin{tabular}{|l|c|c|}
\hline Filter & $\begin{array}{c}\text { Main lobe width }(\mu \mathrm{s}) \\
\pm 10 ~ n s\end{array}$ & $\begin{array}{c}\text { Right side lobe }(\text { dB below main lobe }) \\
\pm 0.1 \mathrm{~dB}\end{array}$ \\
\hline Unfiltered & 707 & 14.6 \\
Sigmoid PI1 & 877 & 13.5 \\
Sigmoid PI2 & 674 & 12.9 \\
\hline Sigmoid PI3 & 568 & 15.0 \\
\hline Sigmoid PI5 & 420 & 13.7 \\
\hline
\end{tabular}

Table 7.1 Main lobe width vs. side lobe suppression

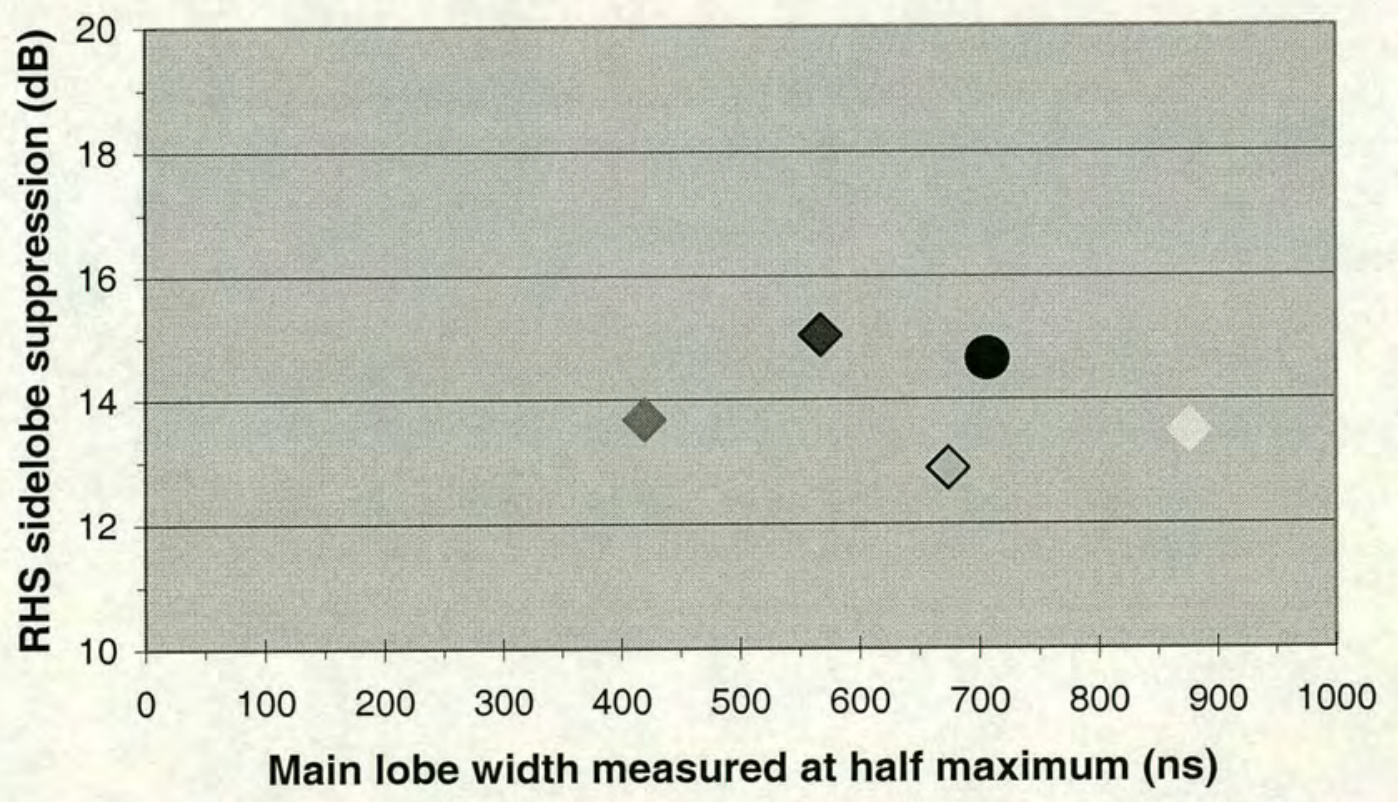

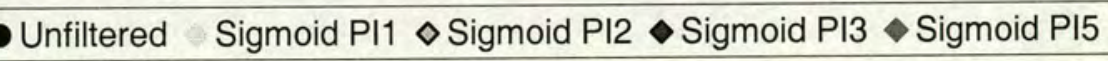

Figure 7.9 Right hand side lobe vs. Main lobe width 


\subsection{Second analysis with neutral phase}

\subsubsection{The problem with signal phase from a phased array}

The analysis of sigmoid based pseudo inverse filters in section 7.4 above is limited by the fact that the axial distance to the phantom is not the same as the axial distance to the near perfect reflector from which the filters were designed. With a phased array system this error will result in significant phase errors (or phase variations), as well as beam spreading (or narrowing) due to focussing, and variations in the locations and frequencies of spectral nulls. Due to the usage of a plane reflector, the beam spreading or narrowing should not be a large source of error.

\section{Phase changes}

The phase changes that occur with propagation in the axial dimension are caused by the complicated interference patterns that are set up by the phased array, and these may cause significant errors in the filtering process. If the phase change of the system impulse response is different from that which the filter is designed for, then the filter will fail to correctly align the phases of the Fourier components. In the worst case the filter could cause pulse spreading by applying incorrect phase changes to the signal.

\section{Spectral nulls}

The variations in spectral nulls that occur with propagation in the axial dimension are also caused by the interference patterns that are set up by the phased array. Spectral nulls will appear at different frequencies at different locations; the spectral nulls that occur at one distance along a given scanline will not necessarily be present at different distances on the same scanline. Thus, if the filter is applied at a different location from where is was designed for, then the filter will incorrectly (or nonoptimally) handle the amplitudes of the Fourier components. 


\subsubsection{A possible solution}

In this section an alternative filter design method (or estimate of the impulse response) is presented for comparison with the filters designed individually for each scanline (in section 7.4). The pseudo inverse filters presented here are Sigmoid based according to equation 4.4 , and the design still uses the data from the near perfect reflection images.

However, in this alternative design the estimate of the system impulse response is altered so that the phase correction (or phase alignment) property of the filter is removed - the filter will not alter the phase of any Fourier component. Also, to avoid the effect of spectral nulls, it is assumed that the underlying spectrum (without interference patterns and associated spectral nulls) is equal at all points along every scanline. This is a plausible assumption since the same transducer elements produce all the scanlines. It is also similar to an approach taken by Delacharte et. al. [112]. To obtain an estimate of the underlying spectrum, the scanlines were averaged; i.e. taking every Fourier component (or frequency) separately, the magnitude of the Fourier components from all of the scanlines were averaged. Due to the limited size of the near perfect reflector used, only about half of the scanlines contribute significantly to the averaged spectrum. However this leaves enough scanlines to obtain a good average with no spectral nulls.

Removing the phase correction property from the filter will make the filter suboptimal, but in this case the filters with the phase correction included are also suboptimal due to the changes in axial distance, so this is a valid comparison to perform. This technique of averaging the scanlines has one further advantage over the previous technique - all scanlines can now be filtered with the same filter, and therefore scan converted images can be reconstructed despite the limitation of the perfect reflector image. 


\subsubsection{Application to the example image}

The filters proposed above were designed and applied to the example phantom image shown in Figure 7.4, using the four Sigmoid based pseudo inverse filter parameter sets presented in Table 4.1. Figure 7.10 shows the example image after being filtered with the filters Sigmoid PI1 and Sigmoid PI3. A visual inspection of the two images in Figure 7.10 shows that for this data there is little difference between the two filters.
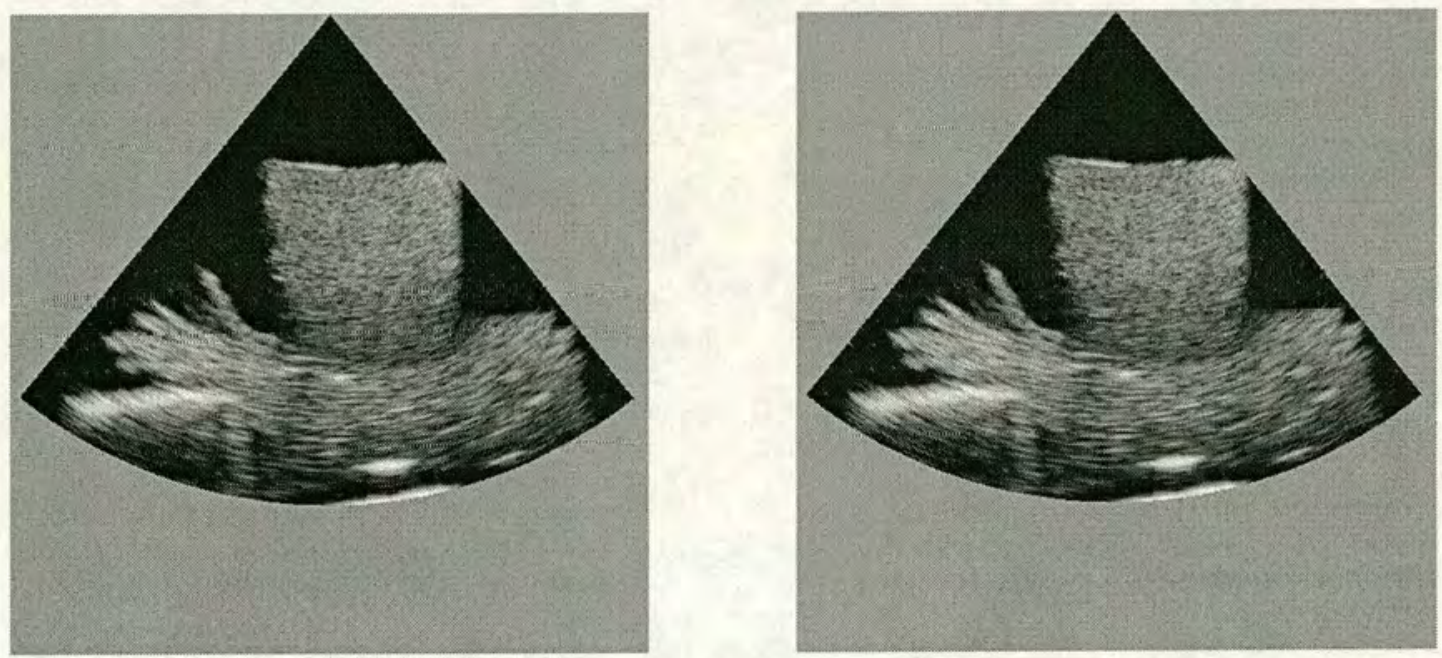

Figure 7.10 The example image filtered with filters Sigmoid PI1 - left, and Sigmoid PI3 - right (designed with an averaged spectrum and no phase correction)

Figure 7.11 shows the $72^{\text {nd }}$ scanline after it was filtered with the filter Sigmoid PI1, and demodulated with a Hilbert transform. Comparing Figure 7.11 with Figure 7.6, it is clear that the main lobe at $t=64 \mu \mathrm{s}$ is narrower than in the unfiltered case when measured $3 \mathrm{~dB}$ below the peak, and is similar when measured 10 to $20 \mathrm{~dB}$ below the peak. The main lobe is narrower than in the case of the filter designed specifically for the $72^{\text {nd }}$ scanline (Figure 7.7). There also seems to be significantly more high frequency components in the demodulated signal produced with this new filter. 


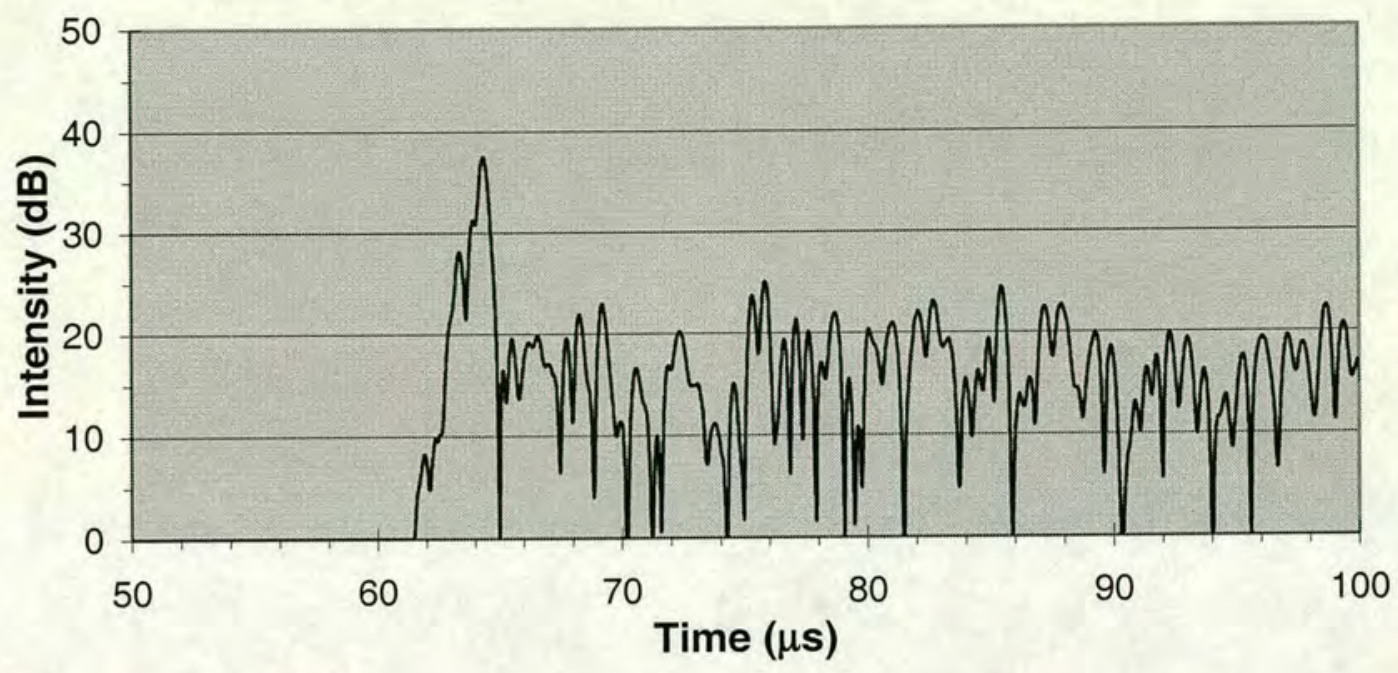

Figure 7.11 The $72^{\text {nd }}$ scanline filtered with Sigmoid PII designed with an averaged spectrum and no phase correction (after demodulation with a Hilbert transform)

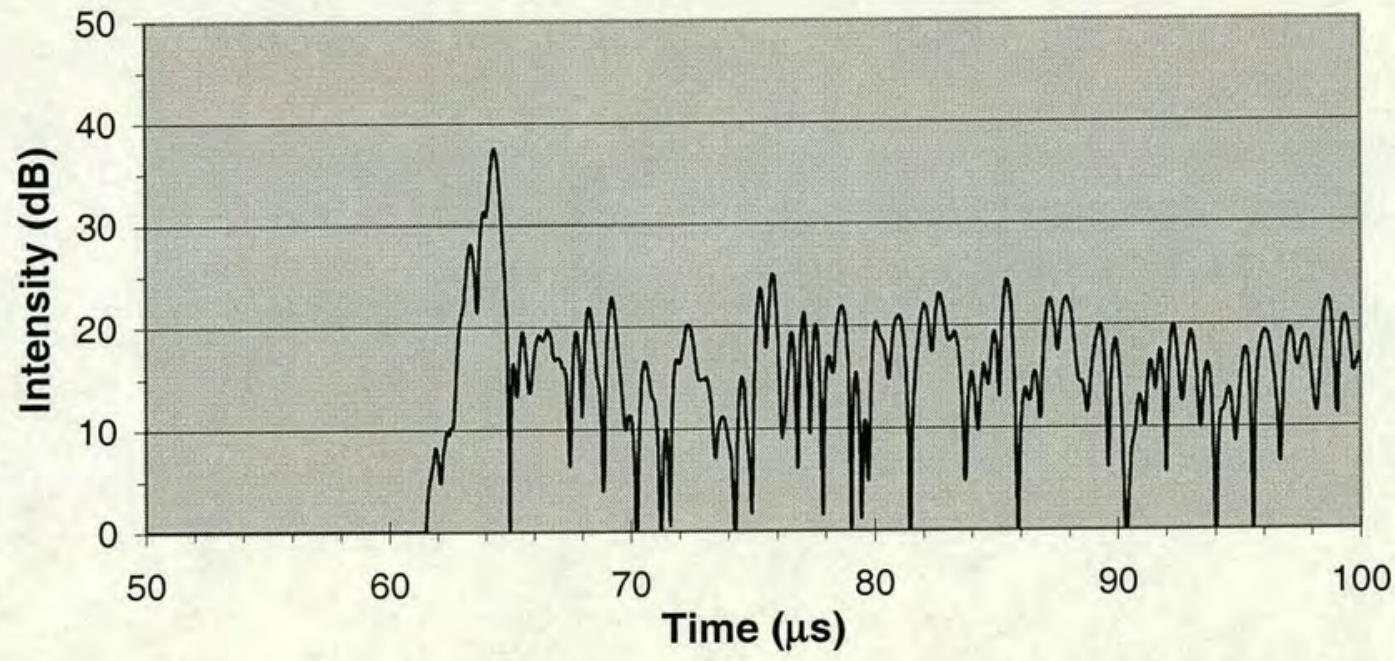

Figure 7.12 The $72^{\text {nd }}$ scanline filtered with Sigmoid PI3 designed with an averaged spectrum and no phase correction (after demodulation with a Hilbert transform)

Figure 7.12 shows the $72^{\text {nd }}$ scanline after it was filtered with the filter "Sigmoid PI3", and demodulated with a Hilbert transform. Comparing Figure 7.12 with Figure 7.5, it is clear that the main lobe at $t=64 \mu \mathrm{s}$ is again narrower than in the unfiltered case when measured $3 \mathrm{~dB}$ below the peak, and is similar when measured 10 to $20 \mathrm{~dB}$ 
below the peak. As with the "Sigmoid PI1" filter, the main lobe is narrower than in the case of the filter designed specifically for the $72^{\text {nd }}$ scanline (Figure 7.8).

\subsubsection{Analysis of main lobe width vs. side lobe suppression}

As with the filters designed specifically for each scanline, the width of the main lobe is measured at the $3 \mathrm{~dB}$ point using linear interpolation between samples, and this is compared with the relative height of the side lobe to right of the main lobe. The measurements are presented in Table 7.2 and are plotted in Figure 7.13.

From Table 7.2 and Figure 7.13 it is clear that all four Sigmoid based pseudo inverse filters have produced a significant improvement in the width of the main lobe narrowing it from around $700 \mathrm{~ns}$ to $400 \mathrm{~ns}$, a reduction of approximately $40 \%$. All four filters have also suppressed the side lobe to the right of the main lobe, by approximately $3 \mathrm{~dB}$. Although this is not a large reduction in the height of the side lobe, it is a significant result that narrowing the main lobe has not resulted in an increase in the side lobe height.

Comparing Figure 7.13 with Figure 7.9 it is apparent that averaging the spectrum and removing the phase correction from the filter has significantly improved the performance of the sigmoid based pseudo inverse filter. This is true for the case of a phased array transducer with image surfaces at differing distances from the perfect reflector distance. It is reasonable to assume that for a single element transducer, this technique will yield no advantages, since the complicated interference pattern that gives rise to the spectral nulls and the large phase changes is due entirely to the interference pattern created by the many separate transmitting elements in the phased array transducer. 


\begin{tabular}{|l|c|c|}
\hline Filter & $\begin{array}{c}\text { Main lobe width }(\mu \mathrm{s}) \\
\pm 10 \mathrm{~ns}\end{array}$ & $\begin{array}{c}\text { Right side lobe (dB below main lobe }) \\
\pm 0.1 \mathrm{~dB}\end{array}$ \\
\hline Unfiltered & 707 & 14.6 \\
\hline Sigmoid PI1 & 429 & 18.0 \\
\hline Sigmoid PI2 & 402 & 18.0 \\
\hline Sigmoid PI3 & 429 & 18.0 \\
\hline Sigmoid PI5 & 411 & 17.2 \\
\hline
\end{tabular}

Table 7.2 Main lobe width vs. side lobe suppression for filters with an averaged spectrum and no phase correction

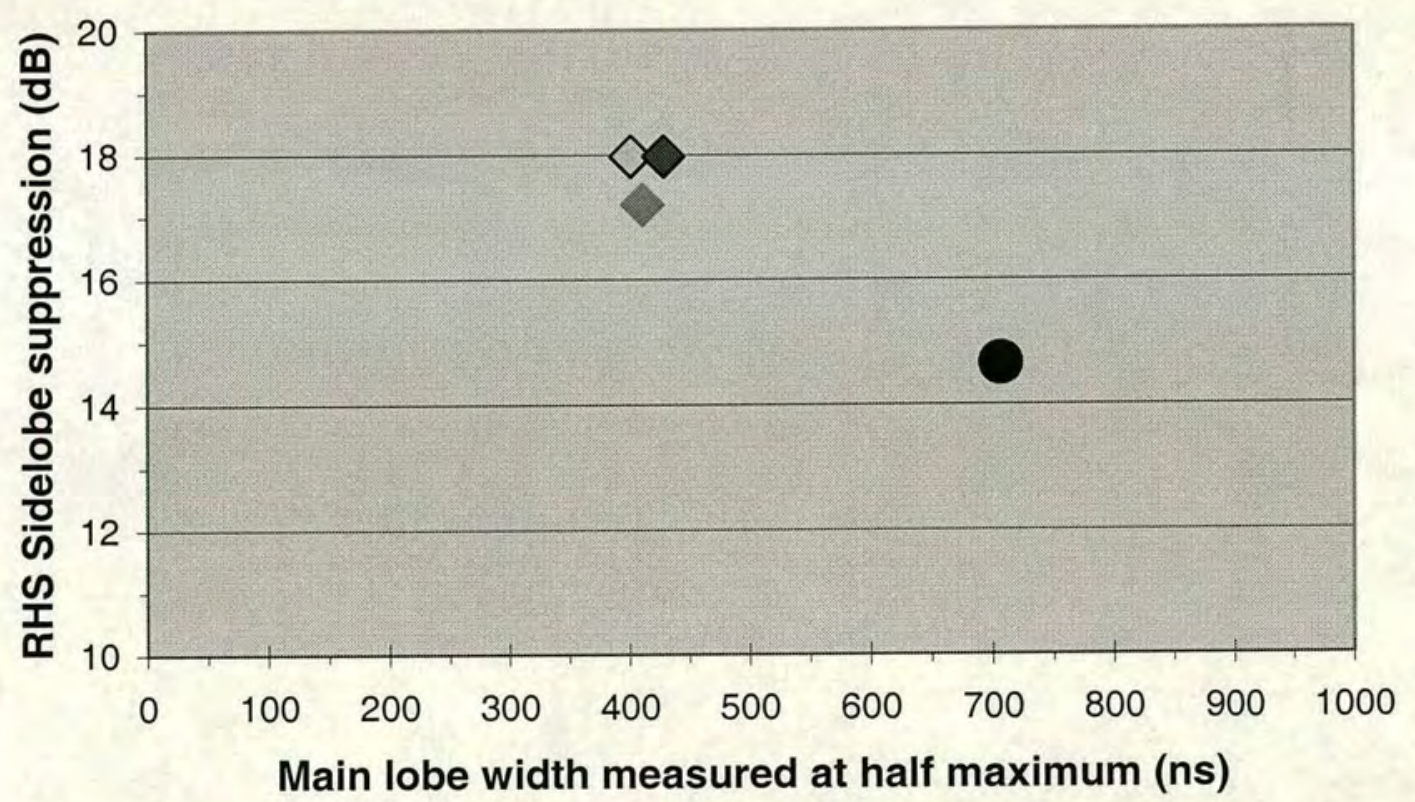

Unfiltered Sigmoid PI1 $\diamond$ Sigmoid PI2 $\diamond$ Sigmoid PI3 $\diamond$ Sigmoid PI5

Figure 7.13 Right hand side lobe vs. Main lobe width for filters designed with an averaged spectrum and no phase correction

Note that the filters Sigmoid PI1 and Sigmoid PI3 are co-incident in Figure 7.13. 


\subsection{Conclusion}

Sigmoid based pseudo inverse filters have been applied successfully to a phantom image obtained with a phased array transducer. They have succeeded in narrowing the width of a lobe representing a reflection from a surface of the phantom when measured $3 \mathrm{~dB}$ below the peak, but they have not narrowed the lobe when measured $10 \mathrm{~dB}$ (or further) below the peak.

A modified sigmoid based pseudo inverse filter has been presented, which is aimed at avoiding the phase and interference problem when applying any filter to a phased array system. It uses no phase correction, and uses the average of several reflections to obtain an estimate of the spectral envelope. This technique removes the need for measuring the impulse response at every axial distance, and has proven to produce results that are favourable (especially when compared to using the impulse response measured at a different axial distance).

This application of the sigmoid based pseudo inverse filter has proven that the filter does indeed provide beneficial results on ultrasound images by improving the resolution of surfaces (or edges). This is a useful result since when applying filters to IVUS images (or any medical images) there exists an uncertainty about the precise structure, shape and texture of the target object. Utilising a phantom has allowed this uncertainty to be removed. 


\section{Chapter 8.}

RESULTS OF

PSEUDO INVERSE FILTERS ON IVUS ULTRASOUND IMAGES

\subsection{Introduction}

This chapter presents the results of applying sigmoid based pseudo inverse filters to images from a medical IVUS system.

The IVUS images presented here are from rabbits, not humans. They are similar to IVUS images of human arteries in most regards, and particularly in that the rabbit arteries are approximately the same diameter as human arteries - hence the structures of interest lie in a similar axial range, and have similar degree of interference with the transducer ringdown. One area where the rabbit IVUS images may be different from images of human arteries is that the rabbit arteries may be less diseased than is typical for images of human patients - since IVUS is seldom performed on humans with healthy arteries. These rabbit IVUS images will provide a realistic test of the applicability of the filters proposed in this thesis.

Comparisons are provided with other filters including the matched, inverse, phase correction, square root based, and Wiener MMSE filters.

\subsection{Image reconstruction procedure}

The images are received as two dimensional uncorrected B-mode images. The filters are applied (in the axial dimension only) to the received signal. The image is then scan converted. Scan conversion is done with; firstly a Hilbert transform for 
demodulation, secondly geometric correction using bilinear interpolation, and thirdly a logarithmic transform. The images presented in this chapter are all reconstructed in exactly the same way (except for the different filters that are applied). All images in this chapter are scaled so that the full range of the image represents a dynamic range of $50 \mathrm{~dB}$.

The geometric transform was performed so that the reconstructed image covered an angle of $361^{\circ}$. Although the reconstructed images covered an area of $360^{\circ}$, it is important for the appearance of the image that there should be no gap between the first and last line. This was avoided by spreading the angles slightly so that there was no blank region remaining. The error introduced by this method is small, and introduces no distortion in the axial dimension (which is the focus of my thesis).

The scan converted images are smaller than the uncorrected (received) image, which means the signal is subsampled during the geometric transform - because of the subsampling, the signal is low pass filtered prior to the geometric transform. The LPF in this case is a simple 8 point summation filter (i.e. an 8 point FIR filter with taps $=\{1 / 8,1 / 8,1 / 8,1 / 8,1 / 8,1 / 8,1 / 8,1 / 8\})$.

\subsubsection{The example image used}

In order to accurately compare the performance of the different filters, each different filter has been applied to the same IVUS image - which is shown in Figure 8.1. This image shows the aorta of a rabbit with a second (branching) artery visible in the lower section of the image.

In Figure 8.1 there are five areas of interest marked. These areas of interest contain features that are clinically useful and are enhanced (or resolved) by some of the filters presented here. The areas highlight the following features:

1. A thin vessel wall.

2. Two transition regions in close proximity. 
3. A thick vessel wall.

4. A double edge - although the second (outer) edge is not visible in Figure 8.1. In this instance the outer edge is not clinically useful but serves as an example of resolving multiple edges in close proximity.

5. An edge, obscured with "speckle". The edge in this instance is not clinically useful, but serves as a test for edge extraction from within "noise".

The widths of arterial walls are clinically important as a measure of the build-up inside the artery.

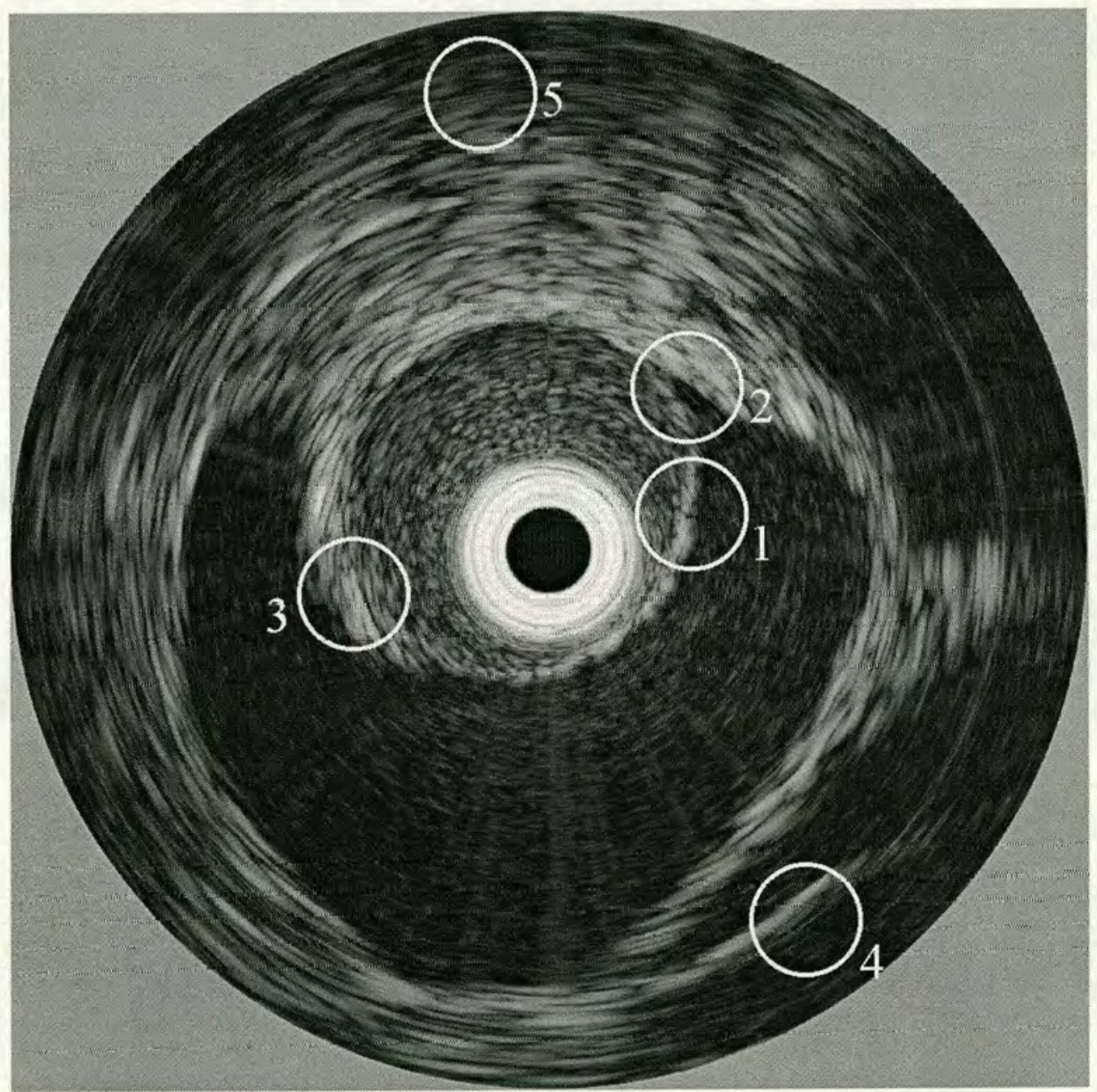

Figure 8.1 The example image with no filtering applied 


\subsection{Regions and lines of interest}

Figure 8.2 shows the example image with five lines of interest highlighted. These lines of interest are selected because they each run through a given region of interest. The lines of interest for each region are 52, 30, 172, 98, and 232 for regions $1,2,3$, 4 , and 5 respectively.

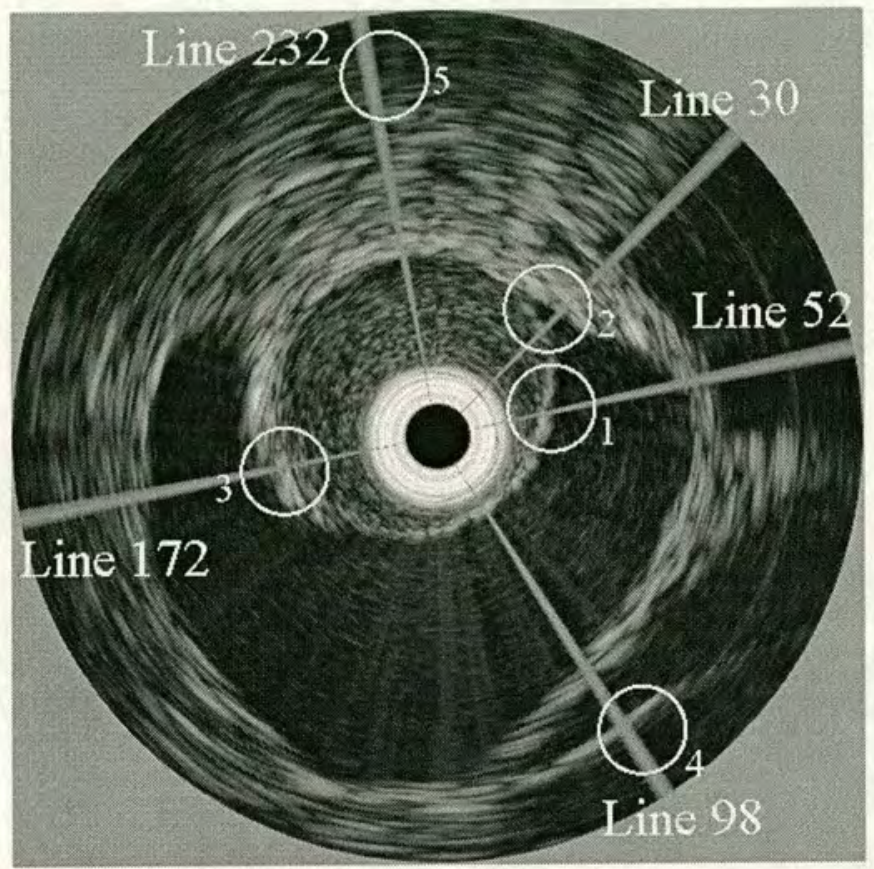

Figure 8.2 Example image with lines of interest highlighted

\subsection{Images filtered with sigmoid based PI filters}

Here the four sigmoid based pseudo inverse filters as defined in Table 4.1 are presented, each applied to the image in Figure 8.1.

Figure 8.3 shows the image of Figure 8.1 after being filtered with the filter Sigmoid PI1. Note that the edge in region 1 appears to be more sharply defined, and hence it is easier to pinpoint its precise location (when compared with Figure 8.1). As will be shown in section 8.6 this apparent increase in edge sharpness is due to the improved 


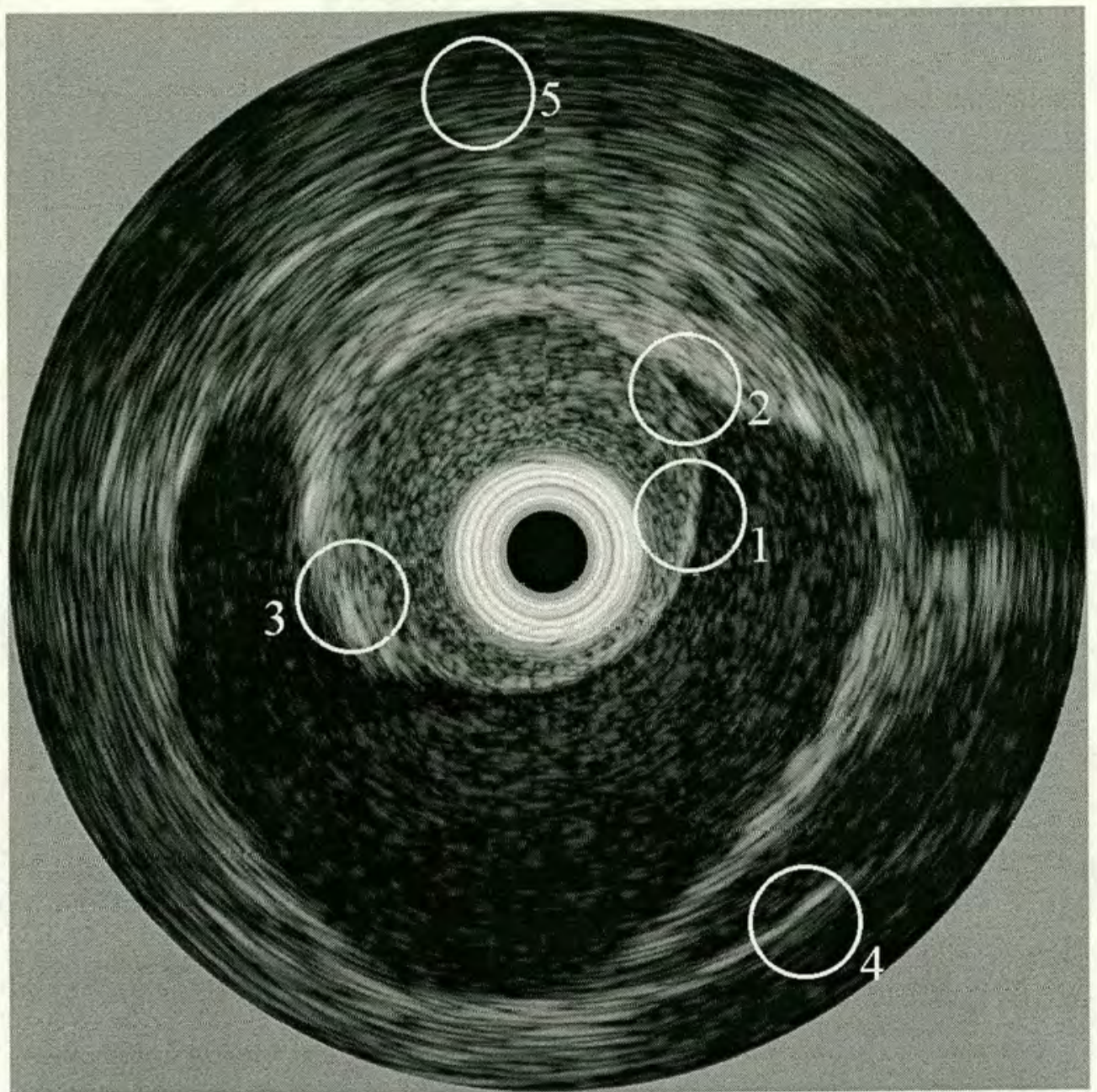

Figure 8.3 Example image after being filtered with filter Sigmoid PII

contrast resolution, and not to the edge itself becoming narrower. In fact the edge has broadened slightly, but the contrast between the regions either side of the boundary has increased. In particular the side lobes immediately beside the edge have been suppressed - by approximately $8 \mathrm{~dB}$ on the right hand side and $1.5 \mathrm{~dB}$ on the left hand side. For visual analysis, this is a clear improvement over the unfiltered image. This improvement (and the importance of contrast resolution) has been verified by visual inspection by cardiologist Dr. Nick Palmer of the Western General Hospital, Edinburgh. However an automated edge extraction algorithm might now locate the edge position with less accuracy due to its extra width. This result suggests that the filter Sigmoid PI1 may have characteristics that are too close to a matched filter for 
this application, and better results may be obtained with filters with characteristics that are closer to an inverse filter.

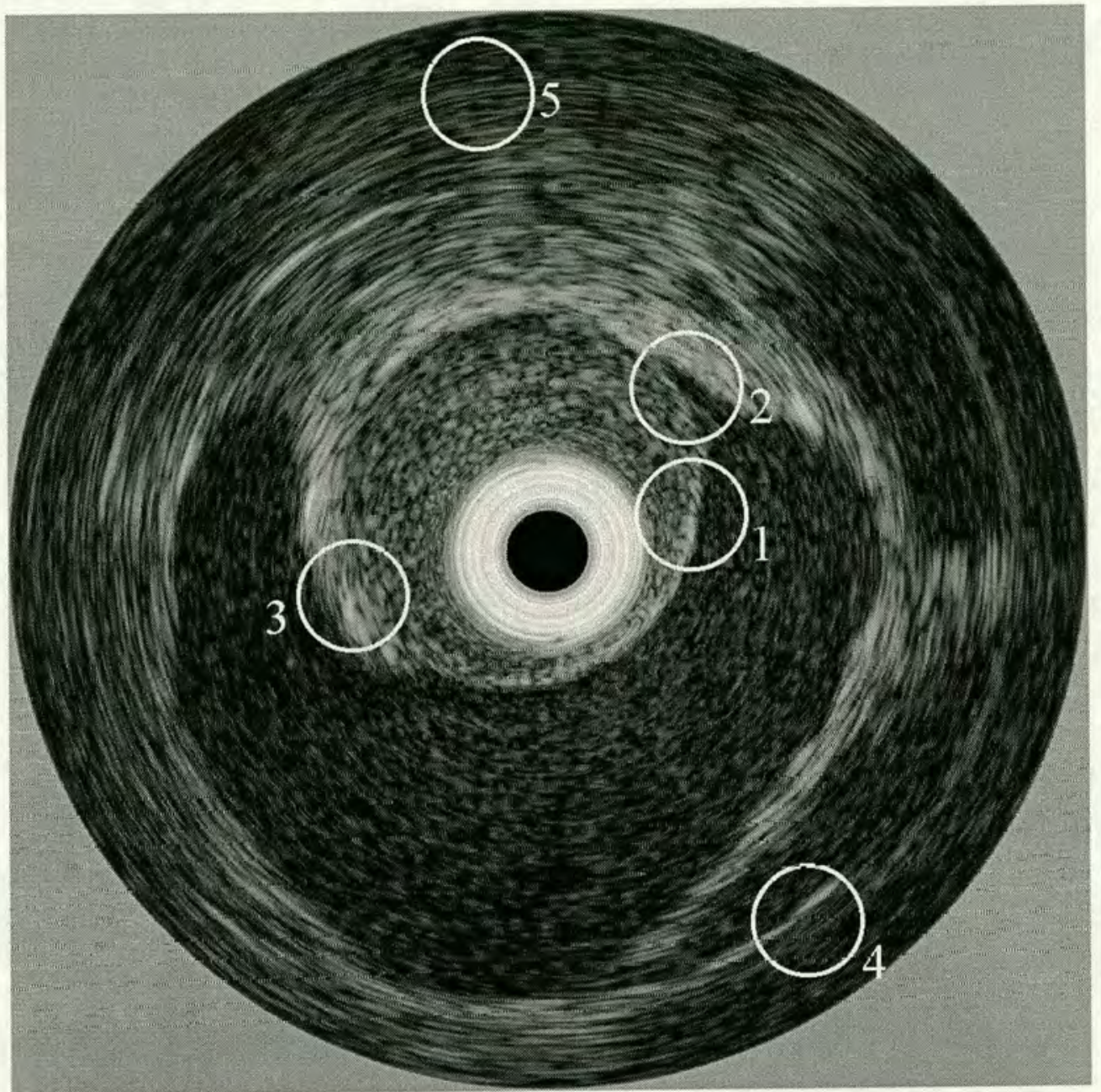

Figure 8.4 Example image after being filtered with filter Sigmoid PI2

In Figure 8.4, the edge in region 1 again appears to be more sharply defined, and hence it is easier to pinpoint its precise location (when compared with Figure 8.1). Like the image in Figure 8.3, the edge is slightly wider than the unfiltered case, and there is also an improvement in the contrast resolution in region 1 . The side lobe immediately to the right of the edge has been suppressed by approximately $3 \mathrm{~dB}$. For visual analysis, this is an improvement over the unfiltered image. For the application of an automated edge extraction algorithm, it is worth noting that although the $3 \mathrm{~dB}$ point the edge is wider than it was in the unfiltered case, when measured at the $1 \mathrm{~dB}$ 
point, the peak has narrowed. Therefore an automated edge extract algorithm should locate the edge position with greater accuracy.

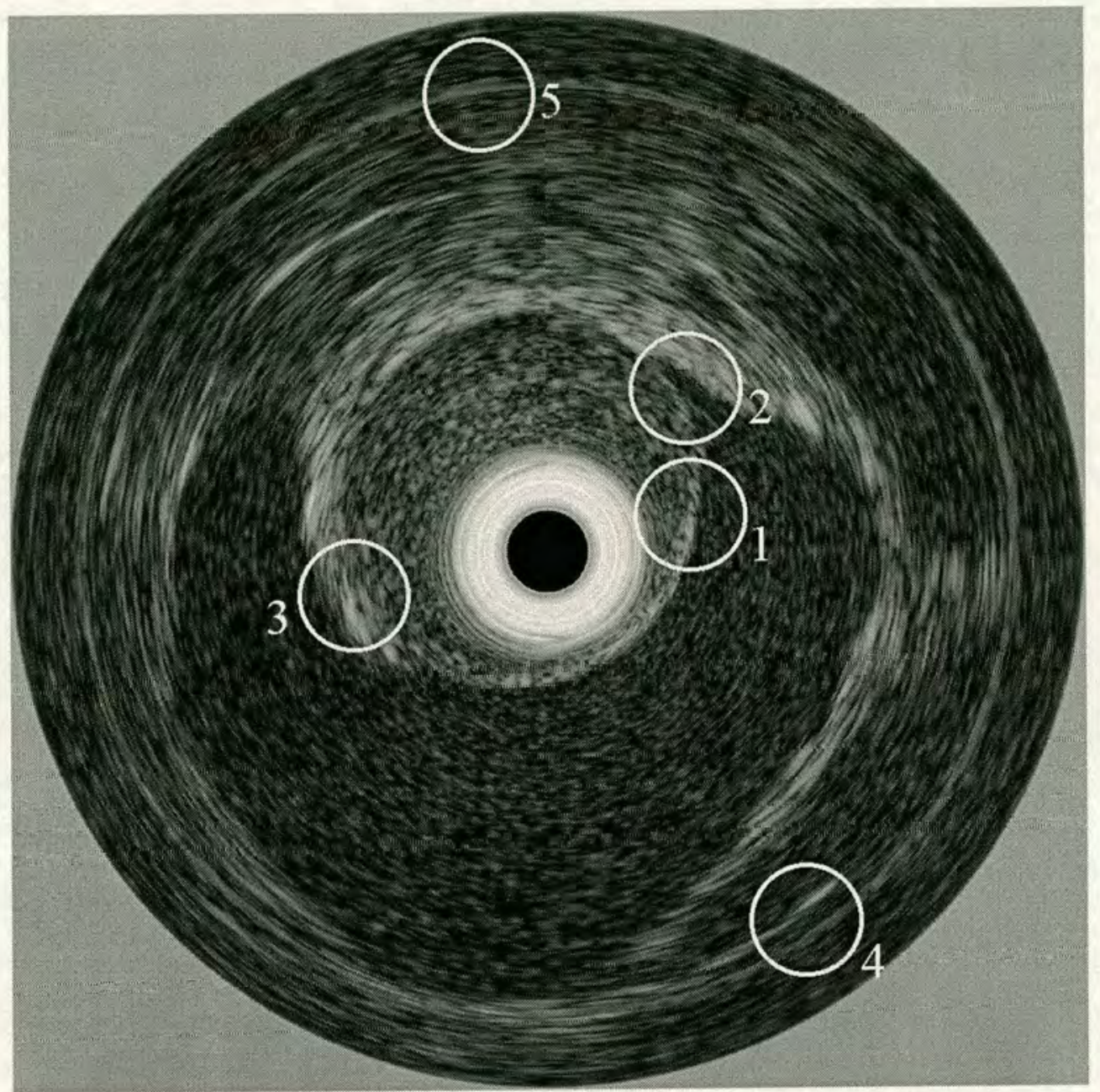

Figure 8.5 Example image after being filtered with filter Sigmoid PI3

In Figure 8.5 , the edge in region 1 again appears to be more sharply defined, and hence it is easier to pinpoint its precise location (when compared with Figure 8.1). Unlike Figure 8.3 and Figure 8.4, there is no improvement in the contrast resolution in region 1. For visual analysis, the precise location of the edge is more clearly determined, however the certainty that the edge exists is not increased over the unfiltered case. The width of the edge when measured at the $3 \mathrm{~dB}$ point is the same as for the unfiltered case. However the width when measured at the $1 \mathrm{~dB}$ point is significantly narrower. Therefore an automated edge extraction algorithm would now 
locate the edge position with greater accuracy due to its reduced width (compared with unfiltered case, or after filtering with Sigmoid PI1, or Sigmoid PI2).

Note that the double edge in region 4 is visible after filtering with Sigmoid PI3. Also, the edge in region 5 is now visible.

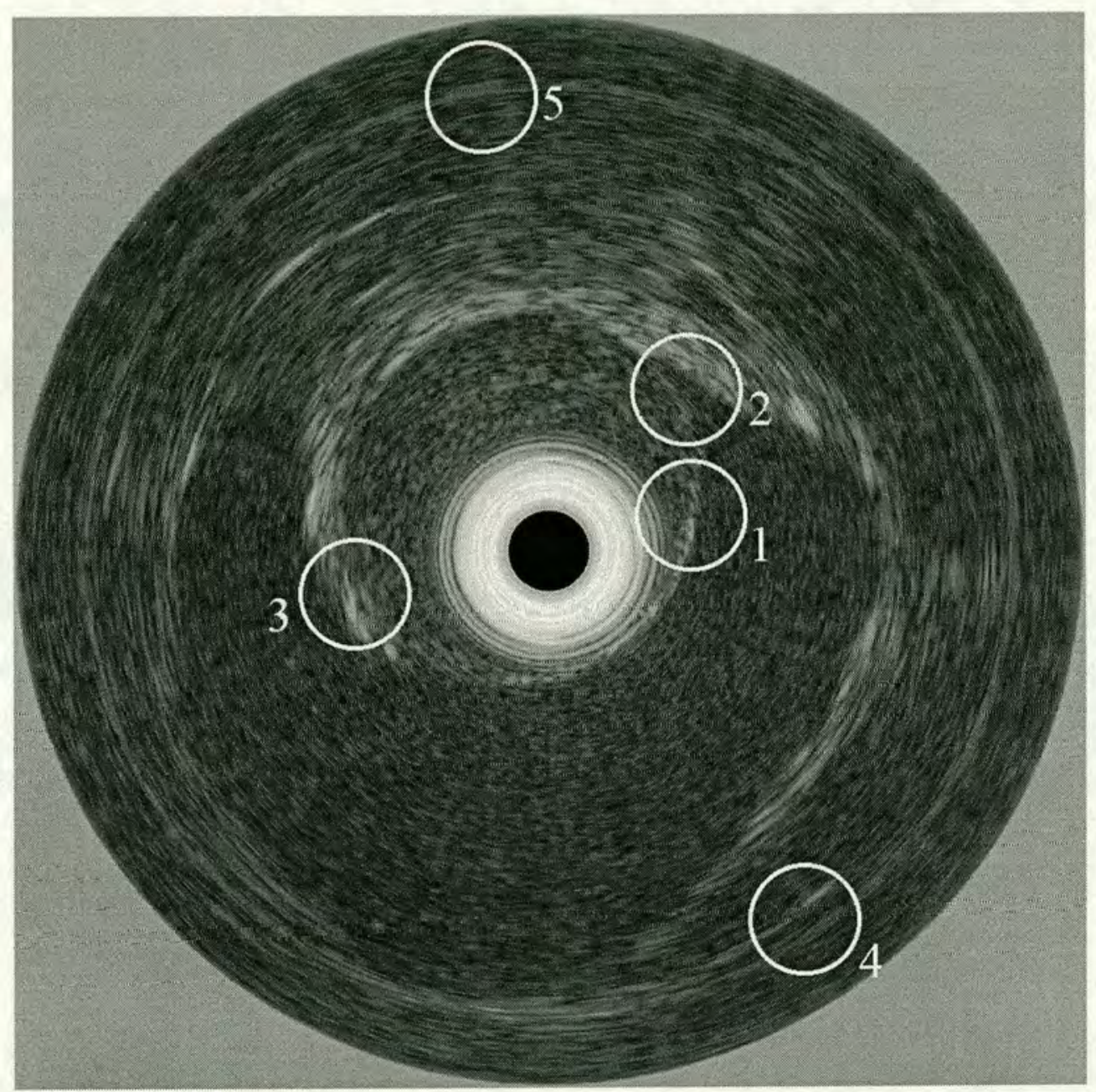

Figure 8.6 Example image after being filtered with filter Sigmoid PI5

In Figure 8.6, the edge in region 1 again appears to be more sharply defined, and hence it is easier to pinpoint its precise location (when compared with Figure 8.1). There is a clear reduction in the contrast resolution in region 1. For visual analysis, the precise location of the edge is more clearly determined, however the certainty that the edge exists is reduced when compared with the unfiltered case. An 
automated edge extraction algorithm would now locate the edge position with greater accuracy due to its reduced width (compared with unfiltered case, or after filtering with the filters Sigmoid PI1, Sigmoid PI2 or Sigmoid PI3). The greatly increased noise level in the image suggests that this filter may have characteristics that are too close to an inverse filter. Better results may be obtained with a filter with characteristics closer to a matched filter.

\subsection{Images filtered with other filters}

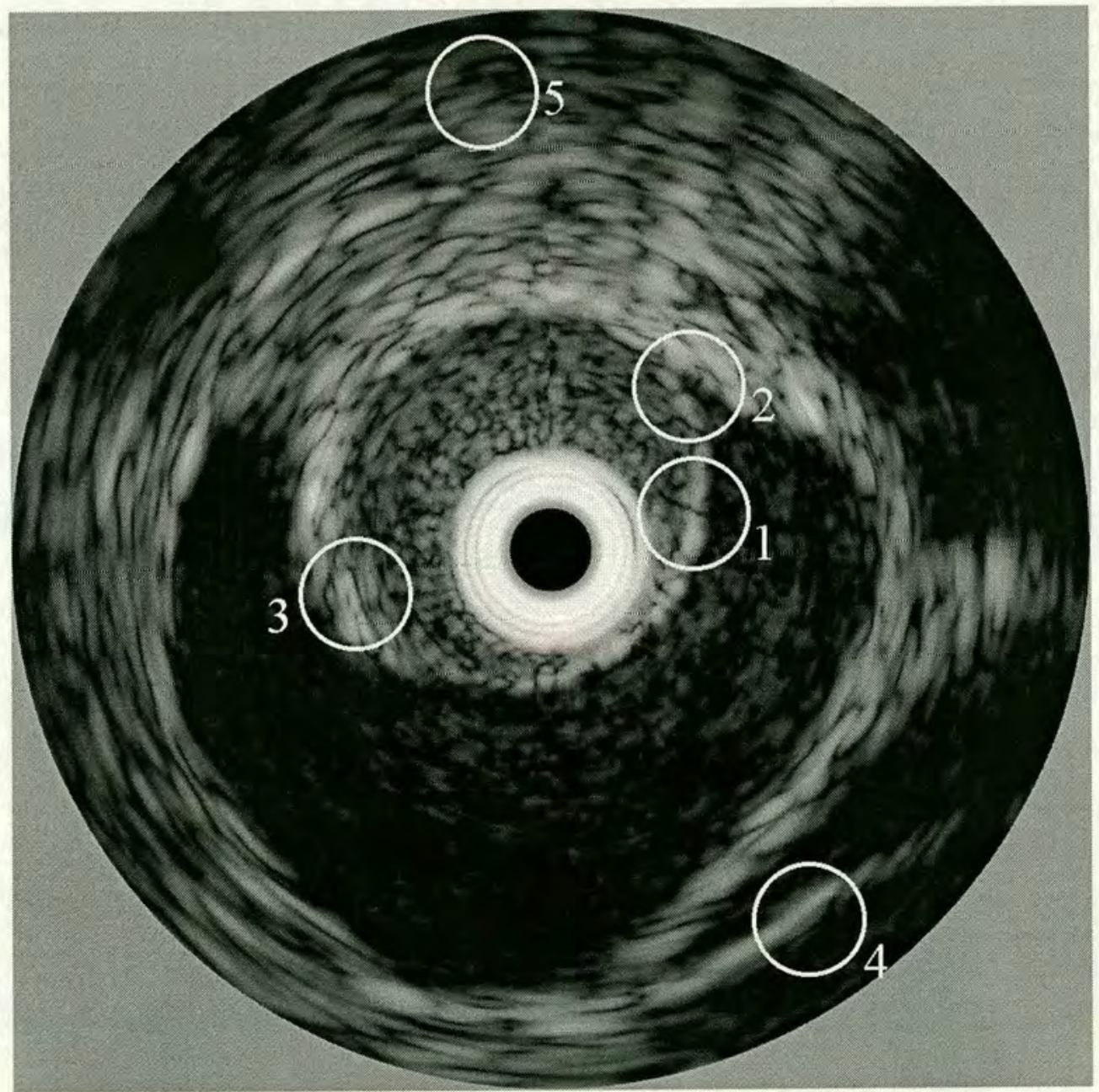

Figure 8.7 Example image after being filtered with a matched filter 
In Figure 8.7, the edge in region 1 appears to be significantly blurred, and hence it is more difficult to pinpoint its precise location (when compared with Figure 8.1). There is a significant increase in the contrast resolution in region 1 . However the significant blurring of the image (when compared with the unfiltered case) means that the matched filter is not viable for ultrasound imaging applications.

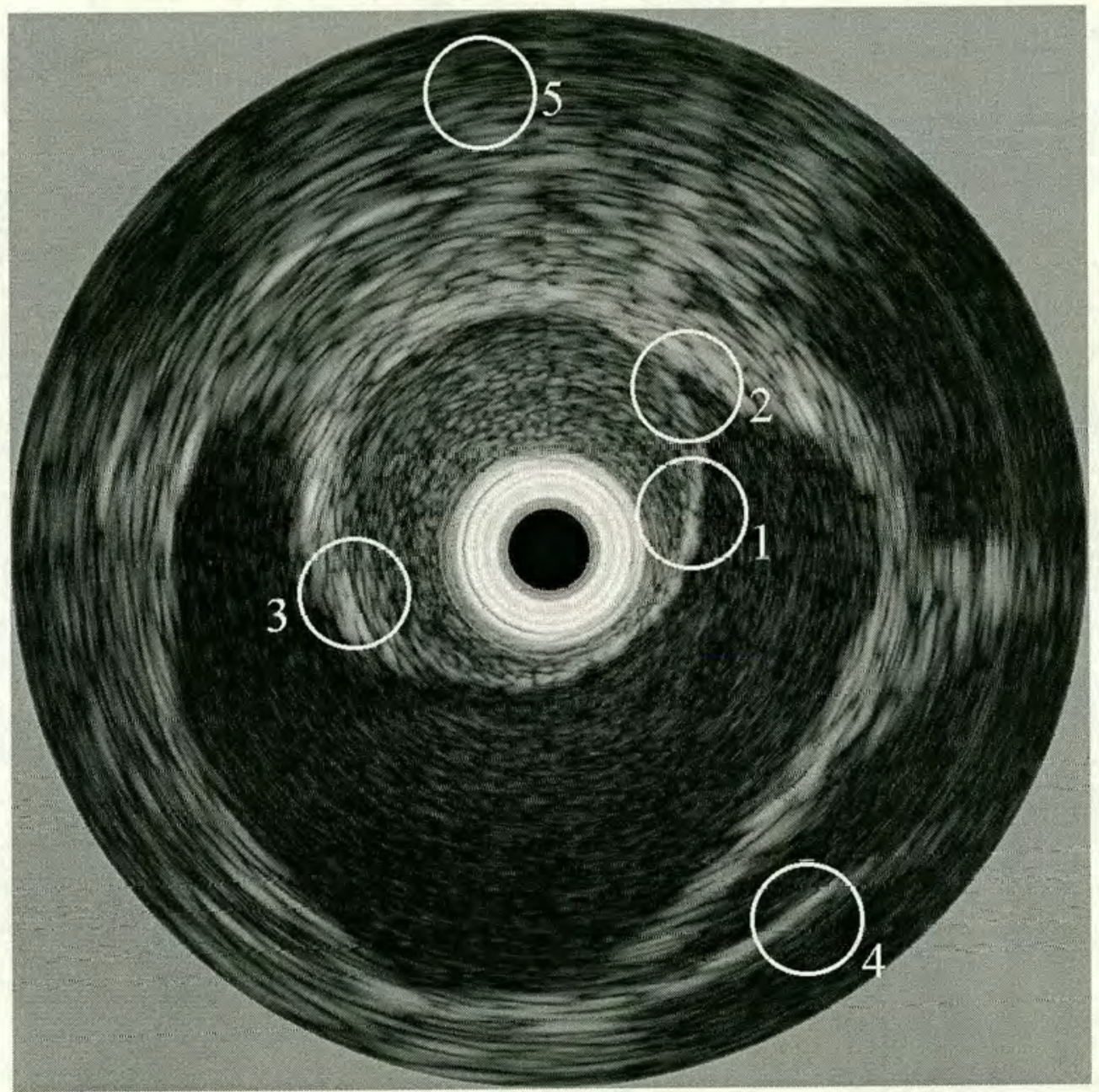

Figure 8.8 Example image after being filtered with a phase correction filter

In Figure 8.8, the edge in region 1 appears to be very similar to the image in Figure 8.1). This indicates that the phase correction or phase alignment characteristic of the filters has not caused any improvement in the image quality. The improvements in the images must therefore be principally due to the amplitude changing effects of the filters. 


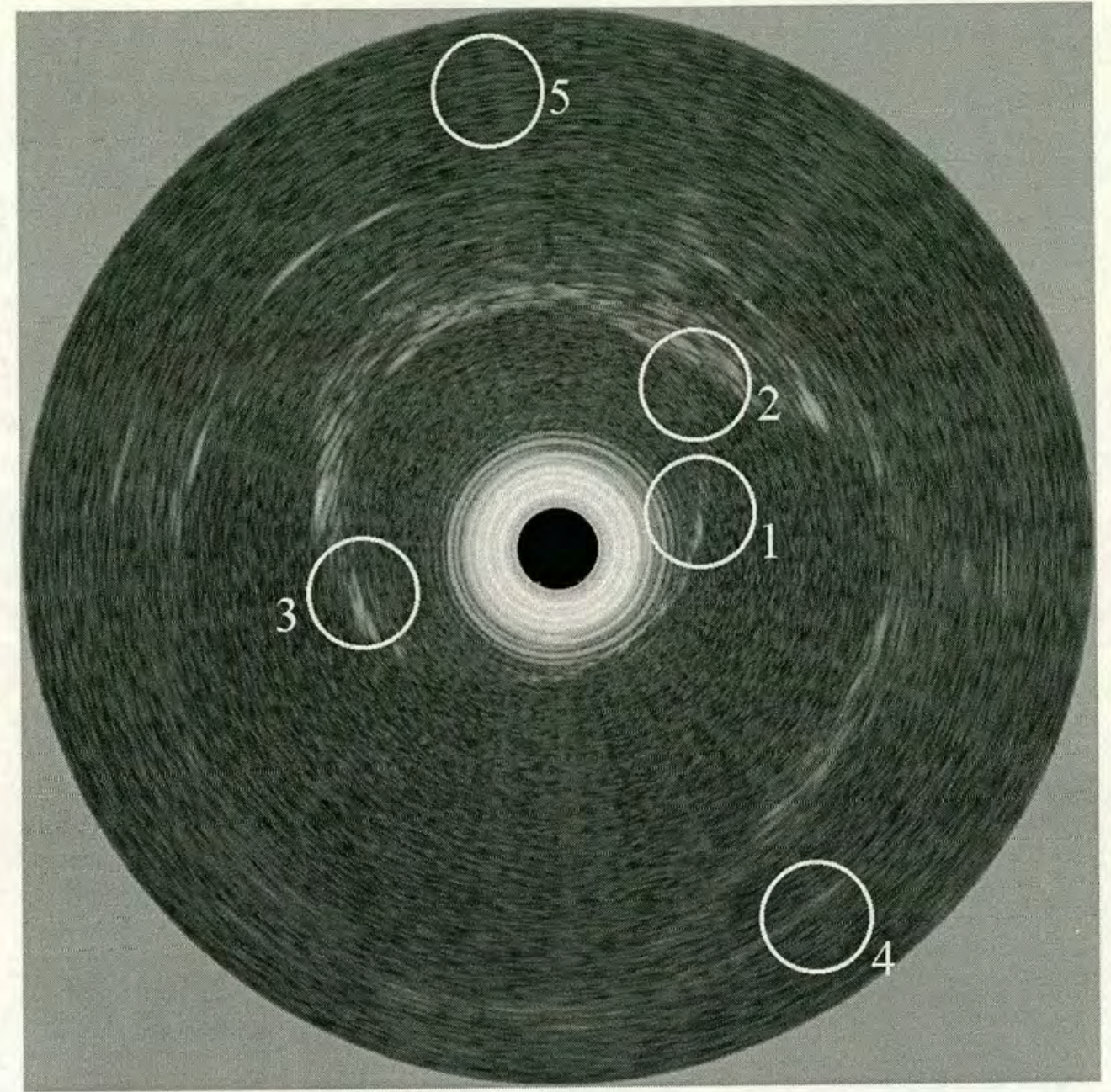

Figure 8.9 Example image after being filtered with a square root based filter

In Figure 8.9, the edge in region 1 is very difficult to see, and hence it is difficult to pinpoint its precise location, and difficult to determine whether the edge exists or not. The contrast resolution in the image is greatly reduced - this result makes this filter a poor choice for ultrasound imaging applications.

The $4^{\text {th }}$ root filter performs similarly to the square root filter, except that the image contrast is reduced further, making analysis even more difficult. 


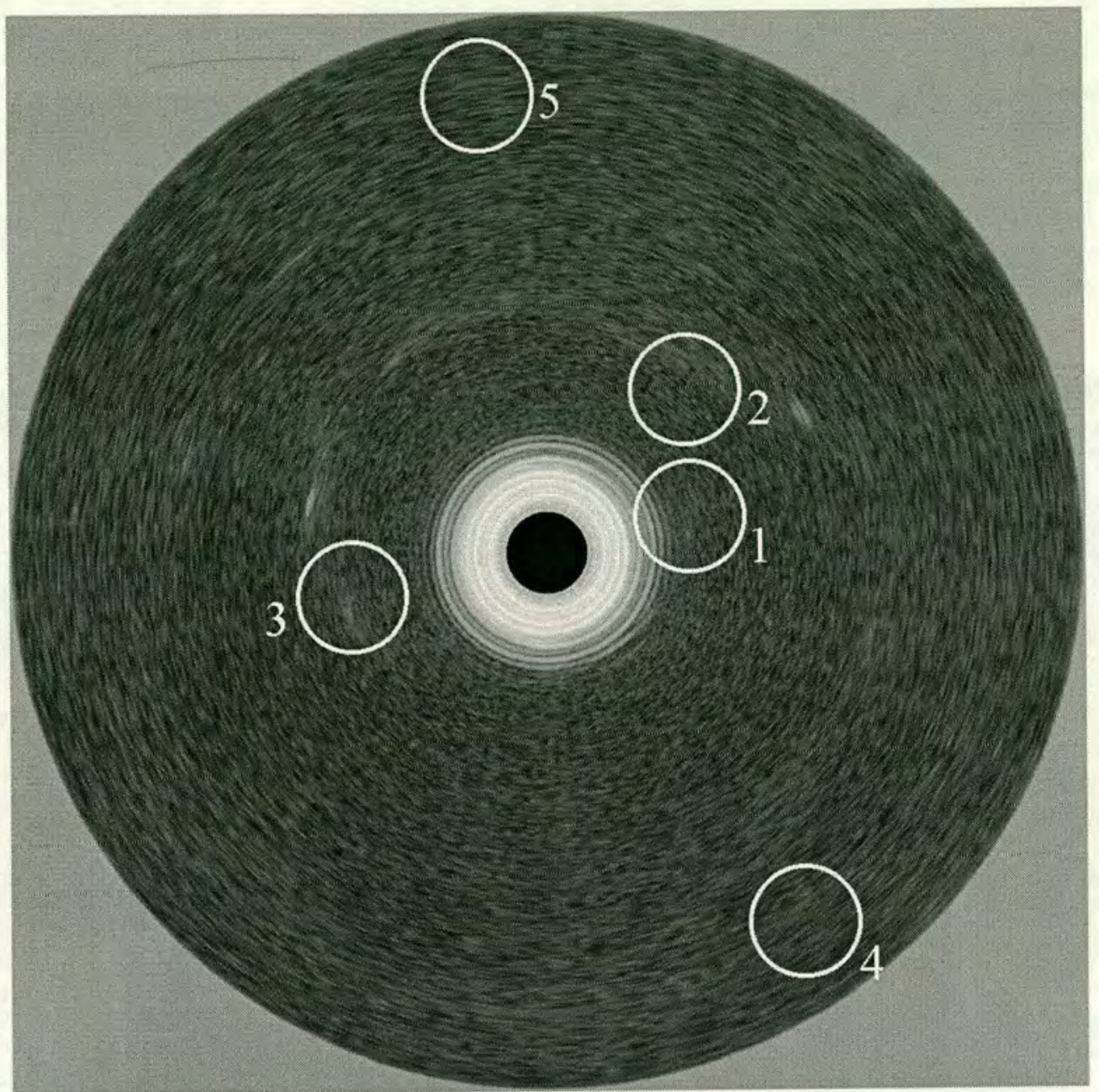

Figure 8.10 Example image after being filtered with an inverse filter

In Figure 8.10, the edge in region 1 is invisible, and hence it is impossible to pinpoint its precise location. The contrast resolution in the image is almost entirely destroyed - this result means that this filter is not viable for ultrasound imaging applications. 


\subsubsection{Images with Wiener filters}

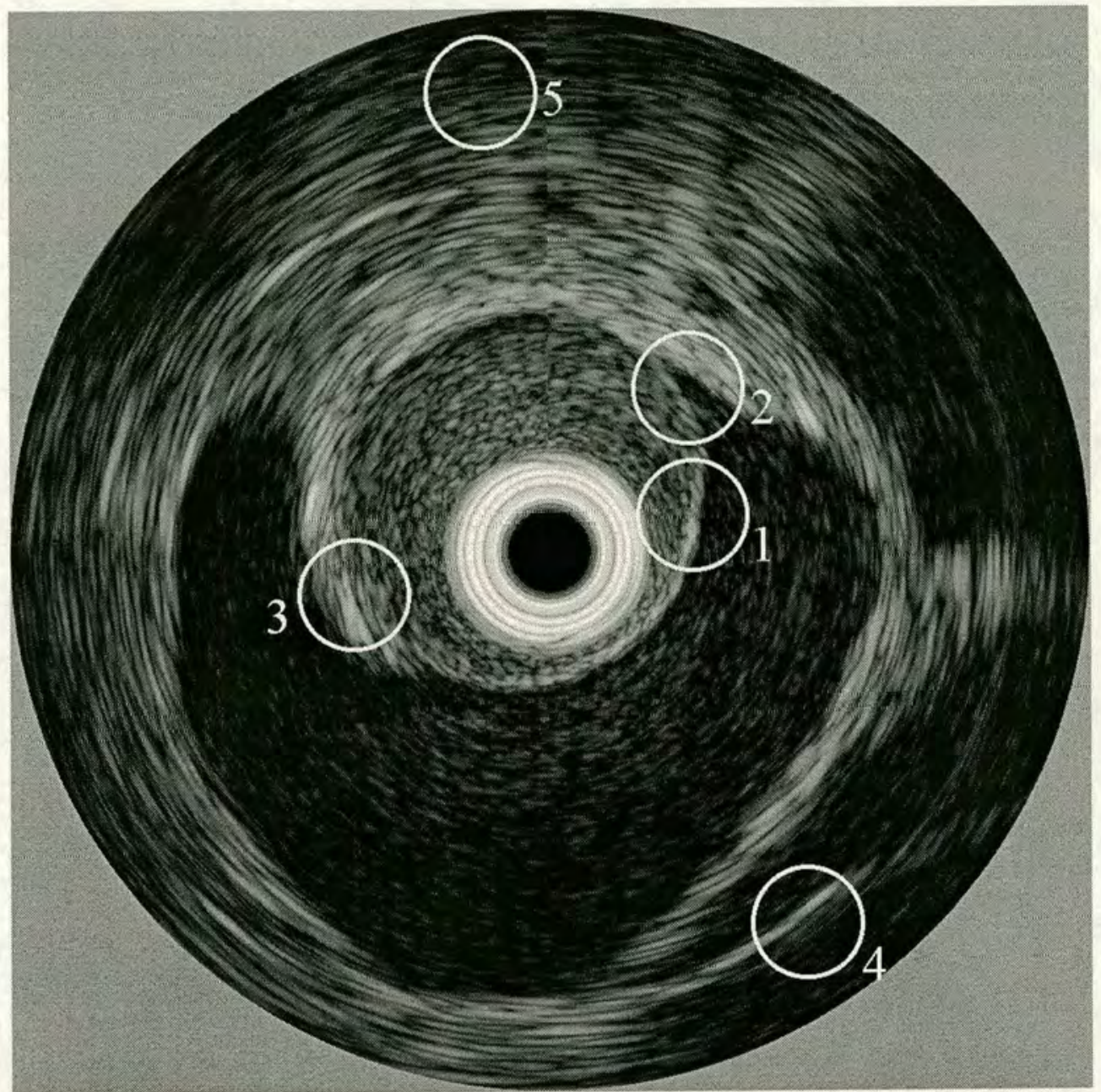

Figure 8.11 Example image after being filtered with a Wiener filter designed for $100 \%$ noise power

The Wiener filter designed for $100 \%$ noise was found earlier to have a performance similar to the filter Sigmoid PI1. The edge width in region 1 has increased slightly but the extra contrast resolution has made the edge more prominent. This is again a similar result to the filter Sigmoid PI1. 


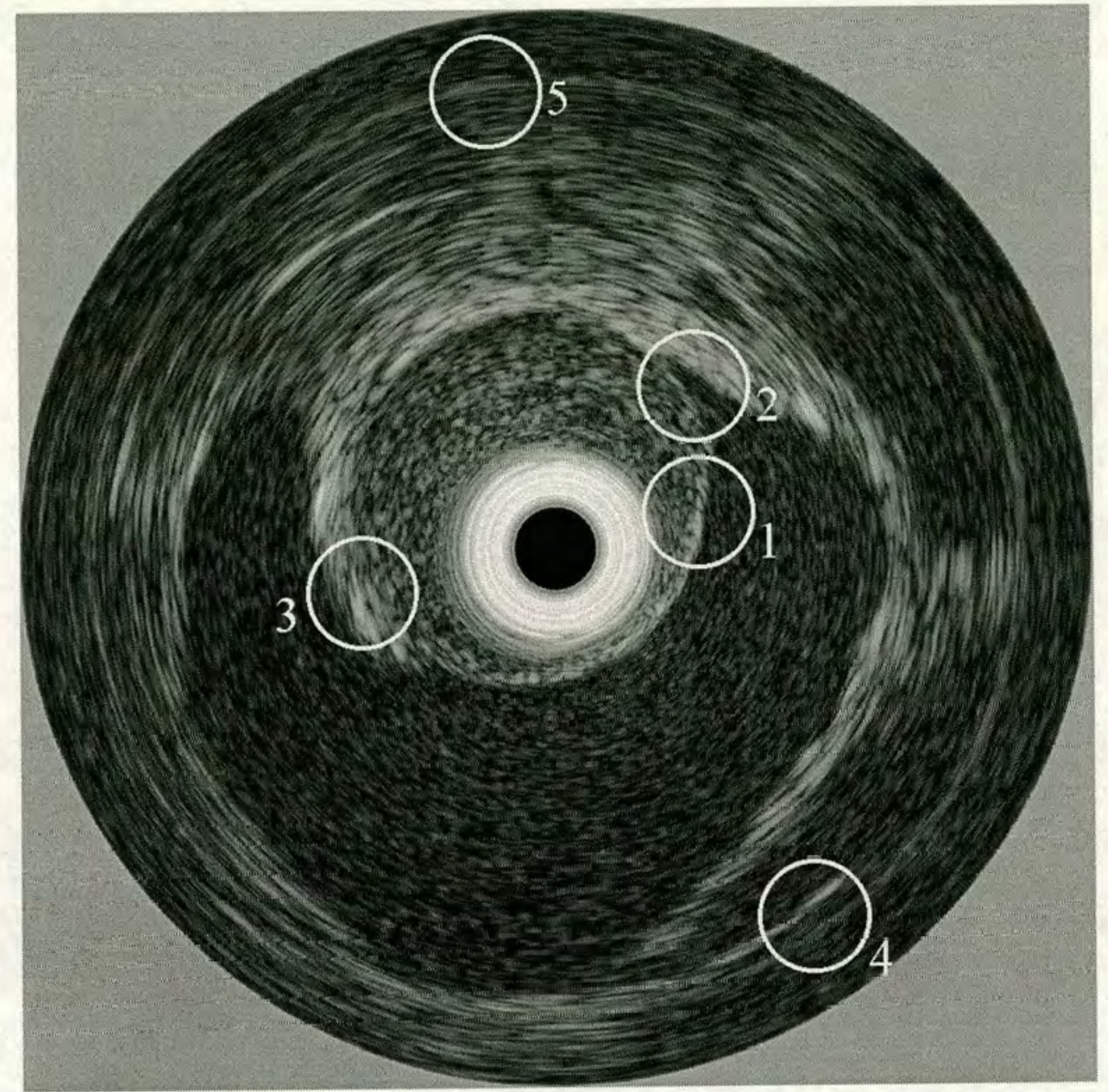

Figure 8.12 Example image after being filtered with a Wiener filter designed for $10 \%$ noise power

After filtering with the Wiener filter designed for $10 \%$ noise, the edge width in region 1 has increased slightly but the extra contrast resolution has again made the edge more prominent. This result is similar to the results of applying the filters Sigmoid PI2 and Sigmoid PI3. 


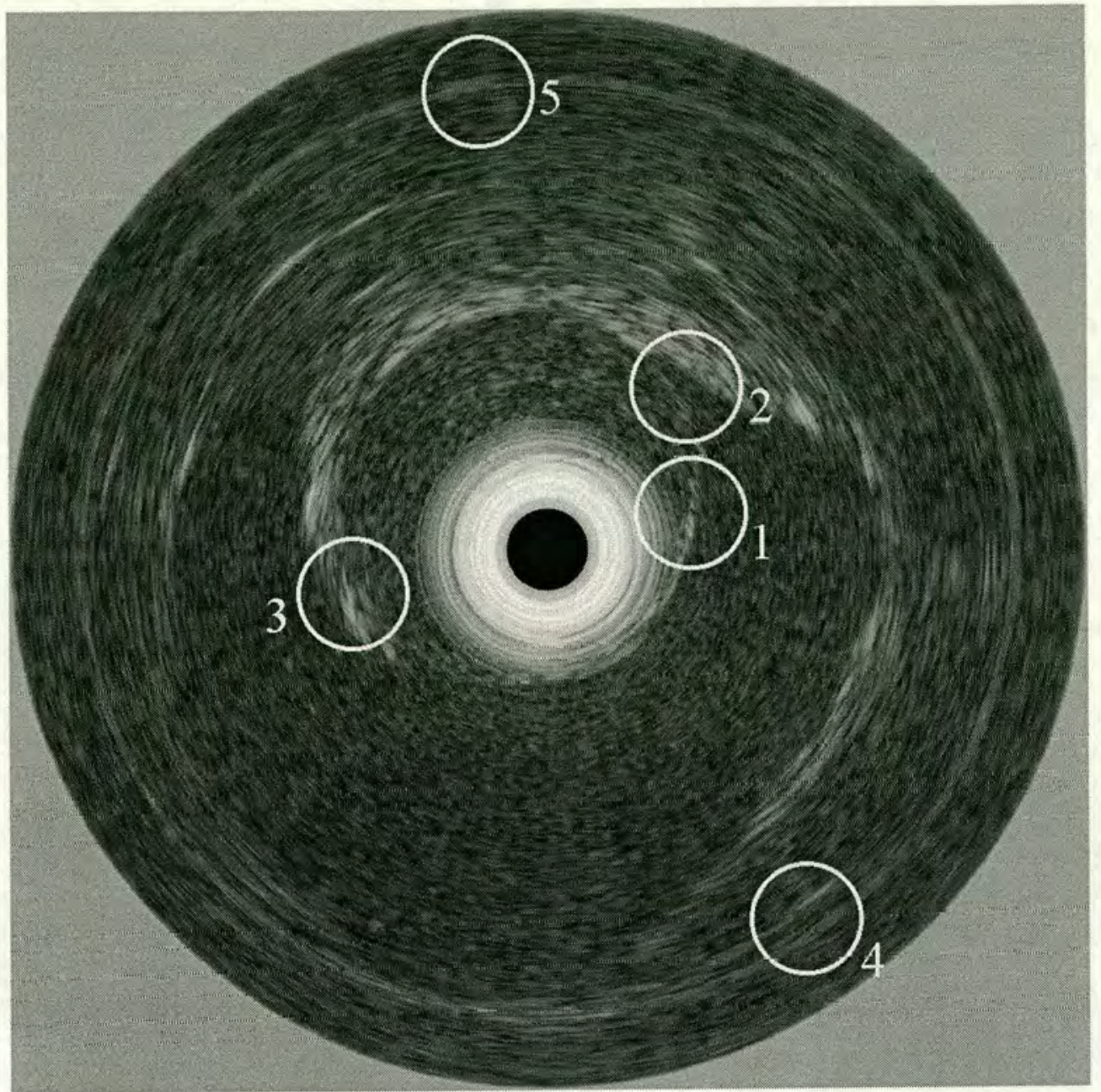

Figure 8.13 Example image after being filtered with a Wiener filter designed for $1 \%$ noise power

After filtering with the Wiener filter designed for $1 \%$ noise, the edge width in region 1 has decreased nearly as much as after filtering with the filter Sigmoid PI5. Also, the contrast resolution has decreased nearly as much as it did after filtering with the filter Sigmoid PI5. This has caused the edge to be less prominent for visual analysis, although its location can be more precisely determined - either visually or by an automated edge extraction algorithm. This is similar to the result of applying the filter Sigmoid PI5. 


\subsection{Thin edge analysis}

Region 1 in the example image (Figure 8.1 to Figure 8.13) contains a thin arterial wall, whose precise location is clinically useful information. This information would also be useful if surfaces were being extracted for constructing a surface rendered 3D image.

Figure 8.14 shows the greyscale intensity (or magnitude) of the reconstructed image with no filtering applied (i.e. Figure 8.1). This shows the $52^{\text {nd }}$ scanline of the received signal with the $\mathrm{x}$-axis covering the range contained within the circle marking region 1 . The $y$-axis is the reconstructed greyscale intensity (or magnitude), where $0-255$ is the total image range, which corresponds to $50 \mathrm{~dB}$.

The feature of interest here is the peak located at time $=0.85 \mu \mathrm{s}$. This is the signal returned from the inner surface of the thin arterial wall.

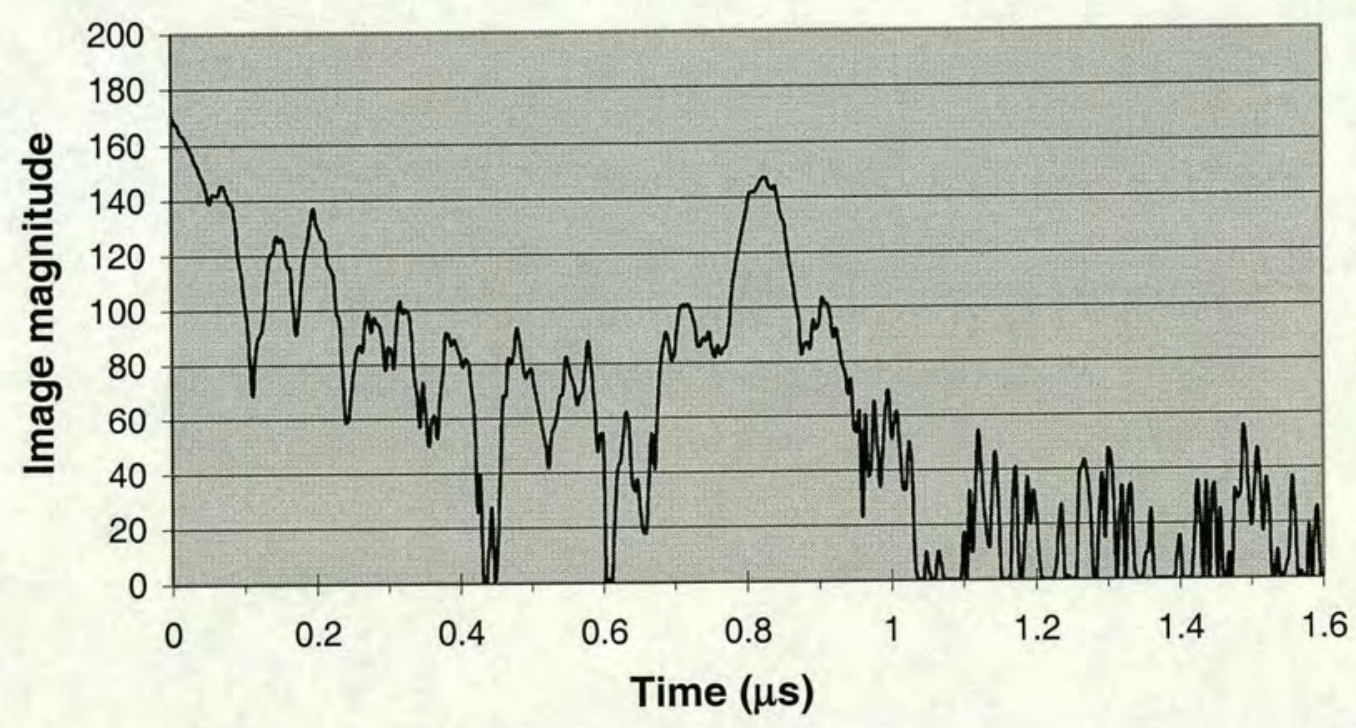

Figure 8.14 Intensity of reconstructed image in region 1 - with no filtering applied 


\subsubsection{Analysis of filter Sigmoid PI1}

Figure 8.15 shows the reconstructed intensity (intensity on the same scanline over the same range as in Figure 8.14) after being filtered with sigmoid based pseudo inverse filter Sigmoid PI1. Note that the filter was applied prior to scan conversion.

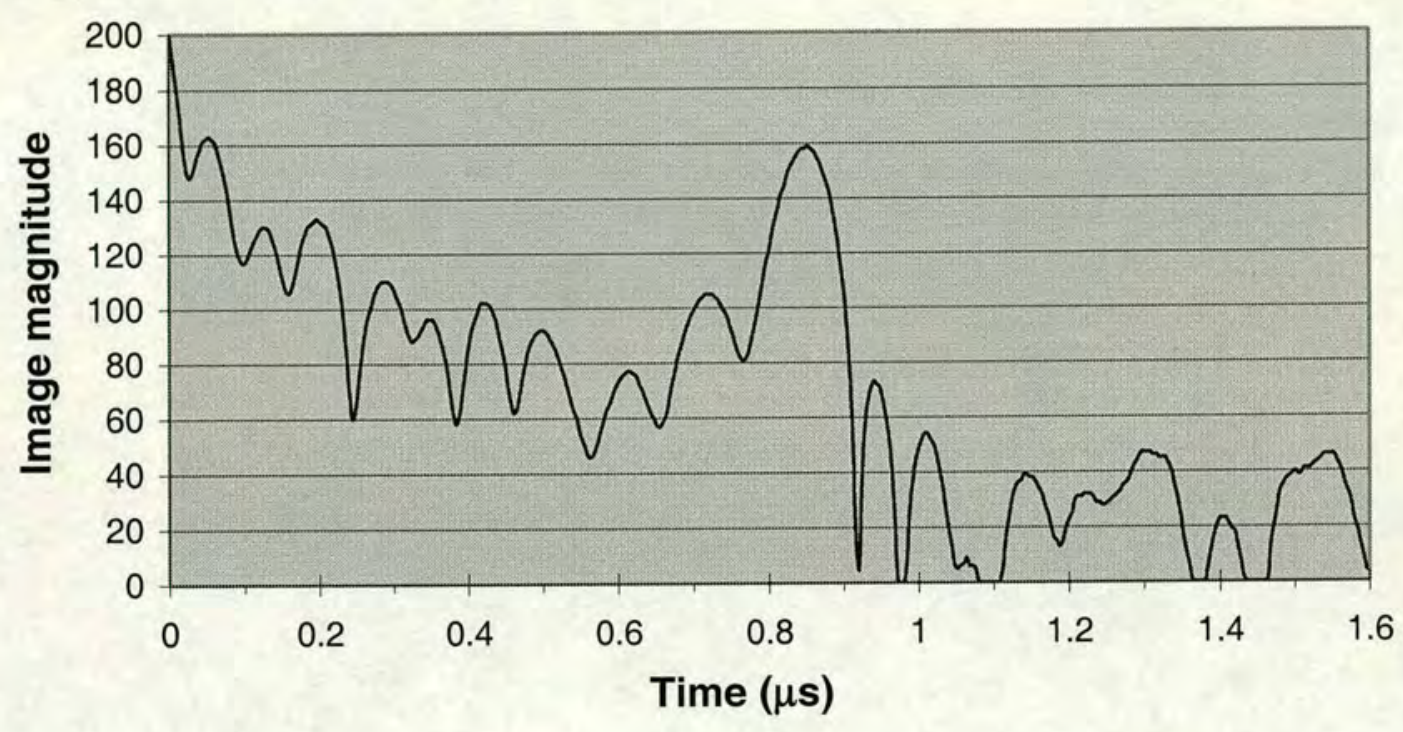

Figure 8.15 Intensity of reconstructed image in region 1 - filtered with filter Sigmoid PII

Comparing Figure 8.15 with the unfiltered case in Figure 8.14, it is apparent that the height of the first side lobe to the right of the main lobe has been reduced in magnitude. In the unfiltered image, the difference in height between the main lobe and the first side lobe on the right is 44 greyscale units or $8.6 \mathrm{~dB}$. $(8.6=44 \times 50 /$ 256). In the image filtered with the filter Sigmoid PI1, the difference is 86 greyscale units or $16.8 \mathrm{~dB}$. On this measure the filtering has made a significant improvement to the image.

It is also apparent that the first side lobe on the left of the main lobe has been reduced in magnitude (with respect to the magnitude of the main lobe). In the unfiltered image the difference between the main lobe and the first side lobe on the left is 46 greyscale units or $9.0 \mathrm{~dB}$. In the image filtered with the filter Sigmoid PI1, the 
difference is 54 greyscale units or $10.5 \mathrm{~dB}$. This is an improvement in the image, but it is of a lesser magnitude than the right hand side lobe.

However in this case, the filtering has increased the width of the main lobe (measured at $3 \mathrm{~dB}$ below the peak) by approximately $15 \%$. This is a reduction in image quality. It is reasonable to conclude that the filter Sigmoid PI1 has characteristics that are significantly removed from an inverse filter; it has broadened the pulse and reduced the noise as a matched filter would do (although a matched filter would broaden the pulse to a much greater extent).

\subsubsection{Analysis of filters Sigmoid PI2, PI3, and PI5}

The following figures present the same region of the example image after being filtered with the filters Sigmoid PI2 (Figure 8.16), Sigmoid PI3 (Figure 8.17), and Sigmoid PI5 (Figure 8.18). As the filters become closer to an inverse filter and more removed from a matched filter, the main lobe width reduces, but the resulting image becomes more noisy and the side lobe magnitude increases (relative to the main lobe).

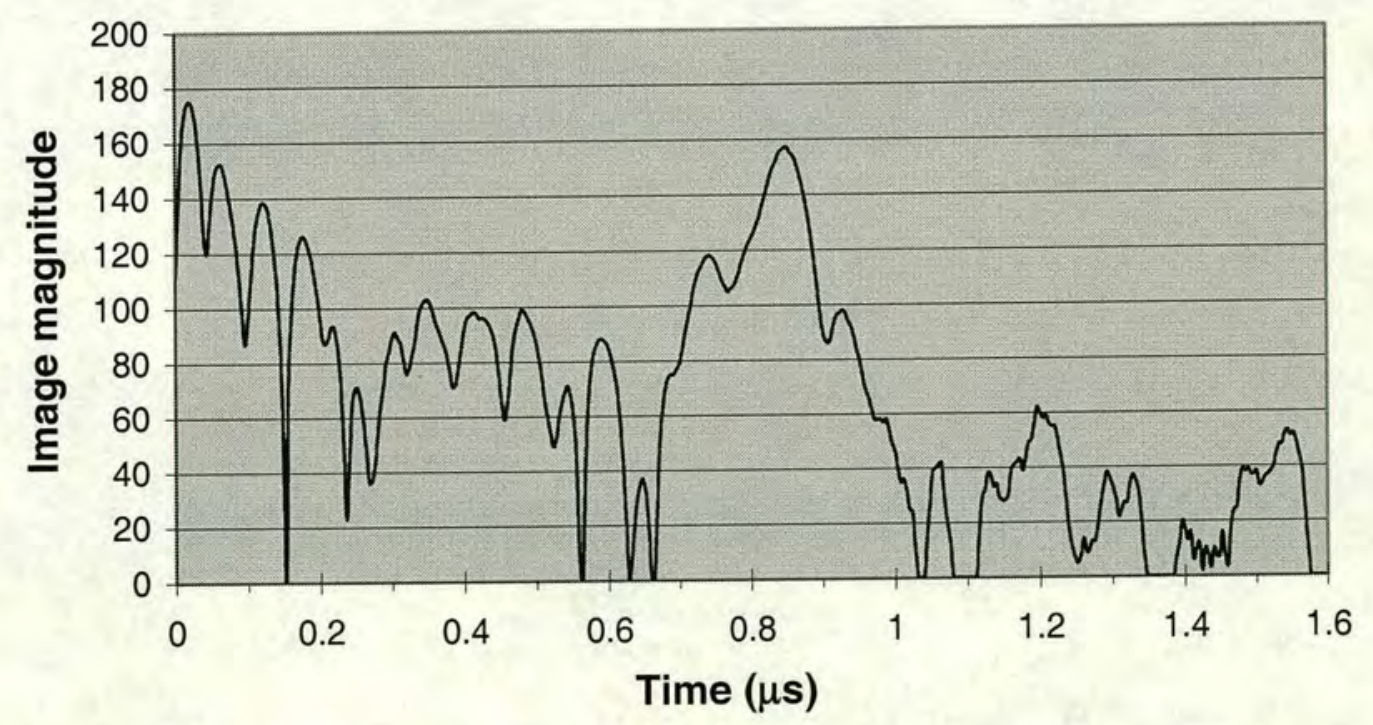

Figure 8.16 Intensity of reconstructed image in region 1 - filtered with filter Sigmoid PI2 


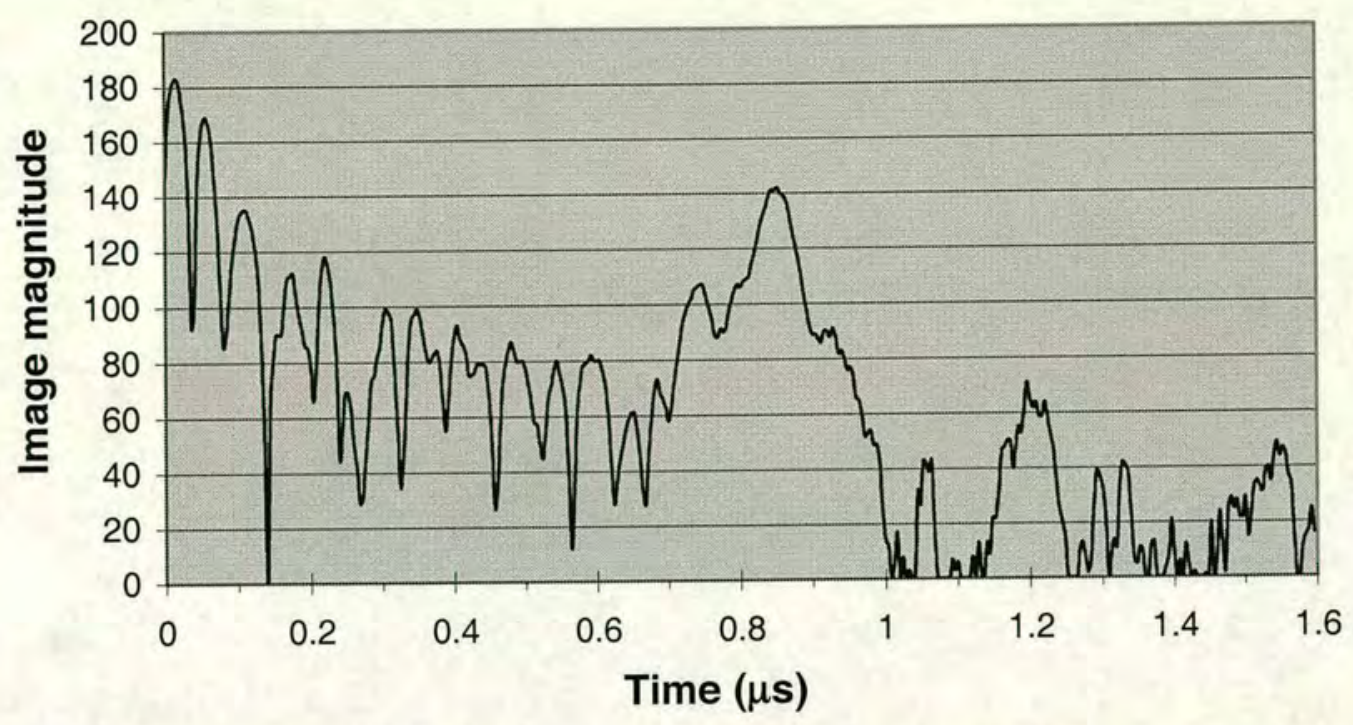

Figure 8.17 Intensity of reconstructed image in region 1 - filtered with filter Sigmoid PI3

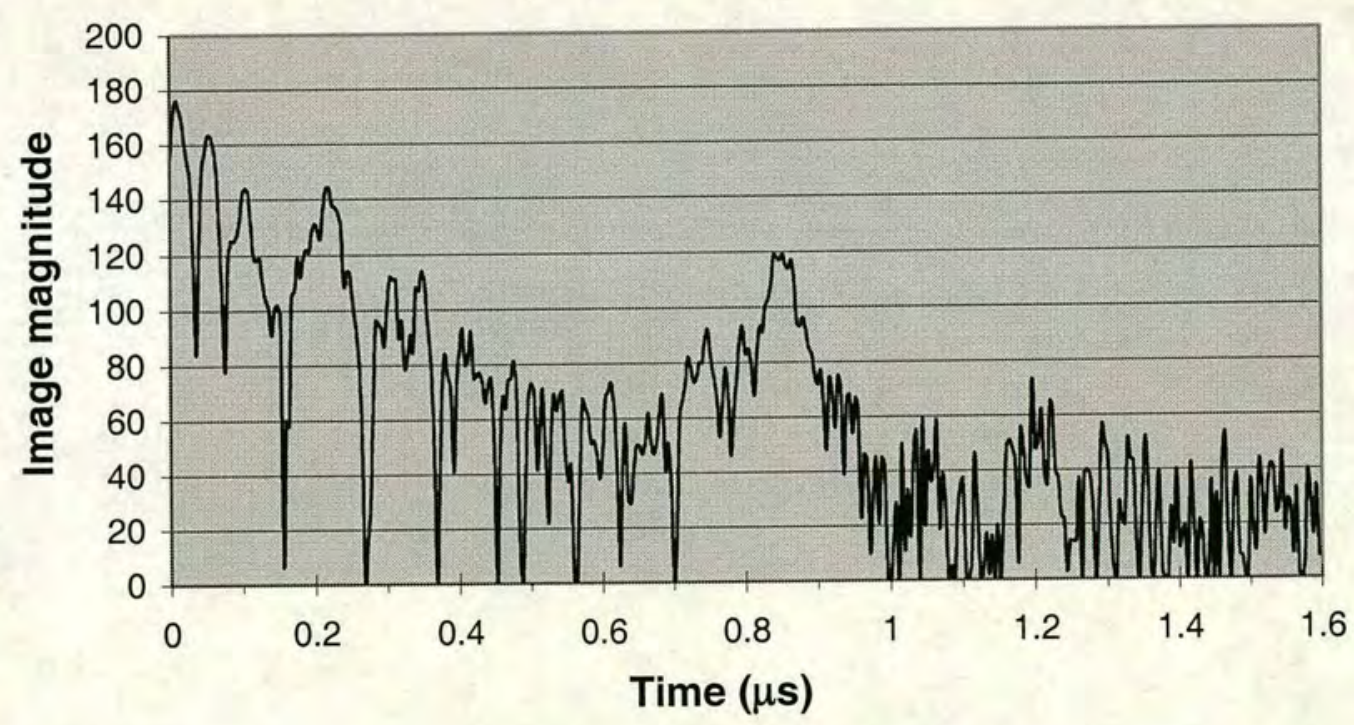

Figure 8.18 Intensity of reconstructed image in region 1 - filtered with filter Sigmoid PI5

\subsubsection{Analysis of main lobe width vs. side lobe suppression}

Pseudo inverse filters are able to trade off the characteristics of a matched filter with an inverse filter. The inverse filter will narrow the main lobe width but increase the 
observed noise level, and the matched filter will reduce the noise level but increase the main lobe width. It is useful to observe how the proposed sigmoid based pseudo inverse filters perform with this trade off. It is also useful to compare the proposed filters with the performance of other filters. The measurements of the main lobe width and the difference in magnitude between the main lobe and the left and right side lobes are presented in Table 8.1. The figures from Table 8.1 are plotted in Figure 8.19 (left side lobe) and Figure 8.20 (right side lobe).

The width of the main lobe is estimated using linear interpolation between discrete sample points (the points are separated by $4 \mathrm{~ns}$ ).

From the results in Figure 8.19 and Figure 8.20 it is clear that on the right hand side lobe, the pseudo inverse filters have made a significant improvement over the unfiltered case. E.g. the filter Sigmoid PI3 yields the same main lobe width as the unfiltered case, but has superior side lobe suppression, and the filter Sigmoid PI5 yields a narrower main lobe but has reduced side lobe suppression (and greater noise levels) than the unfiltered case.

For the left hand side lobe, the improvement over the unfiltered case is not dramatic - the unfiltered case lies above the line of the pseudo inverse filters. This means that although the pseudo inverse filters can trade off main lobe width with noise or side lobe suppression, their performance is such that they cannot get a better "suppression/lobe width" ratio than the unfiltered case.

In comparison with other pseudo inverse filters, the sigmoid based pseudo inverse filters performs creditably. The filter performs better than the square root and $4^{\text {th }}$ root based filters, and performs almost as well as the Wiener filters. 


\begin{tabular}{|c|c|c|c|}
\hline Filter & $\begin{array}{c}\text { Main lobe width }(\mu s) \\
\pm 1 n s\end{array}$ & $\begin{array}{l}\text { Left side lobe ( } \mathrm{dB} \\
\text { below main lobe) } \\
\quad \pm 0.1 \mathrm{~dB}\end{array}$ & $\begin{array}{c}\text { Right side lobe ( } \mathrm{dB} \\
\text { below main lobe) } \\
\pm 0.1 \mathrm{~dB}\end{array}$ \\
\hline Unfiltered & 50.6 & 9.0 & 8.6 \\
\hline Sigmoid PI1 & 60.2 & 10.5 & 16.8 \\
\hline Sigmoid PI2 & 52.3 & 7.6 & 11.5 \\
\hline Sigmoid PI3 & 50.6 & 6.8 & 10.0 \\
\hline Sigmoid PI5 & 34.9 & 5.0 & 4.5 \\
\hline
\end{tabular}

Table 8.1 Main lobe width vs. side lobe suppression

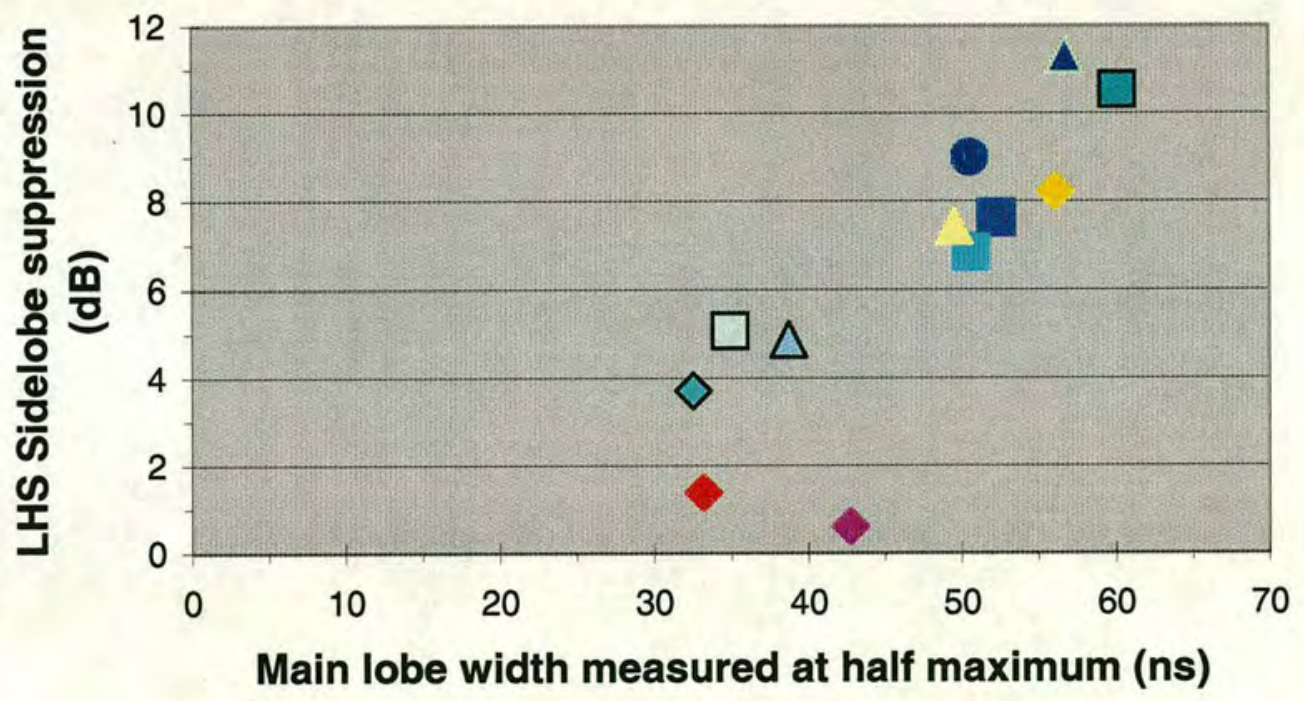

\begin{tabular}{|c|c|c|c|}
\hline - Unfiltered & Phase & $\diamond$ Sqrt & 4th Root \\
\hline 口PI5 & Wiener $100 \%$ & Wiener $10 \%$ & $\Delta$ Wiener $1 \%$ \\
\hline
\end{tabular}

Figure 8.19 Left hand side lobe vs. Main lobe width ${ }^{*}$

\footnotetext{
" In Figure 8.19 and Figure 8.20 the matched filter is not plotted, because after matched filtering the
} main lobe was broadened to the extent that the side lobes were no longer distinguishable. 


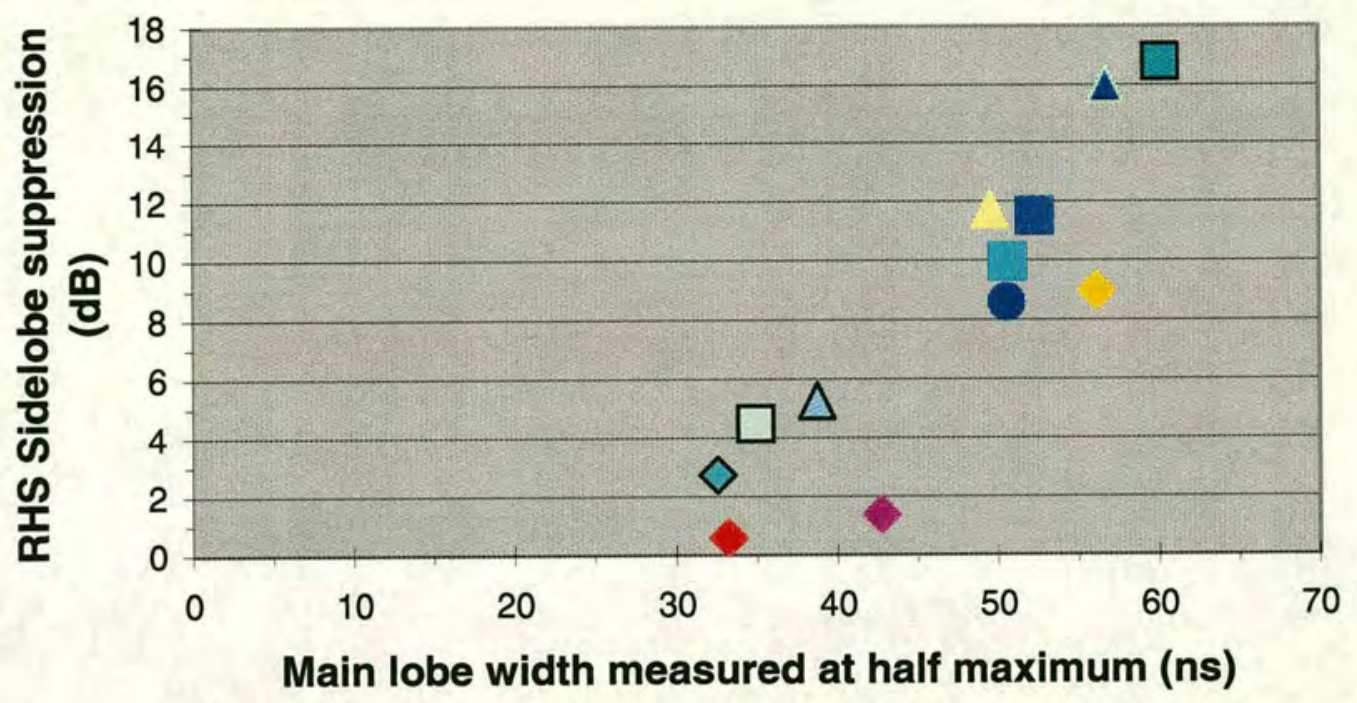

\begin{tabular}{llll}
\hline Unfiltered & Phase & $\diamond$ Sqrt & 4th Root \\
Inverse & $\square \mathrm{PI} 1$ & $\mathrm{PI} 2$ & $\mathrm{PI3}$ \\
$\square \mathrm{PI5}$ & $\star$ Wiener100\% & Wiener10\% & $\Delta$ Wiener1\% \\
\hline
\end{tabular}

Figure 8.20 Right hand side lobe vs. Main lobe width

\subsection{Double edge analysis}

The separation of double edges, or surfaces, is an important criteria of image resolution. Region 4 in the example image (Figure 8.1 to Figure 8.13), shows a double edge which some of the pseudo inverse filters have been able to separate.

Figure 8.21 shows the greyscale intensity of the reconstructed image with no filtering applied (i.e. corresponding to Figure 8.1). This shows the $98^{\text {th }}$ scanline of the received signal with the $\mathrm{x}$-axis covering the range contained within the circle marking region 4 . The $y$-axis is the reconstructed greyscale intensity, where $0-255$ is the total image range, which corresponds to $50 \mathrm{~dB}$.

The features of interest here are the major peak located at time $=0.45 \mu \mathrm{s}$, and the secondary peaks such as those located at time $=0.35 \mu \mathrm{s}$, and at time $=0.57 \mu \mathrm{s}$. The secondary peaks are not clearly separated in Figure 8.21 - but they are after application of some pseudo inverse filters. 


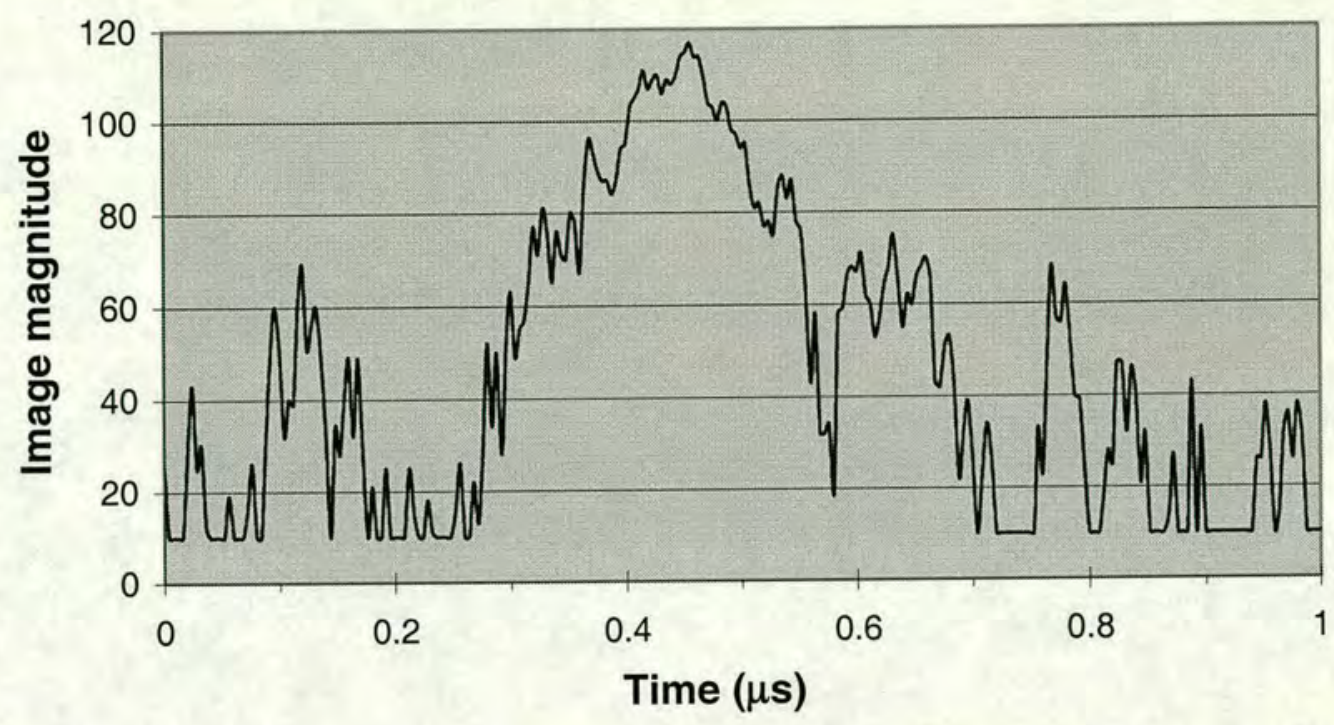

Figure 8.21 Intensity of reconstructed image in region 4 - with no filtering applied

The following figures present the same region of the example image after being filtered with the filters Sigmoid PI1 (Figure 8.22), Sigmoid PI2 (Figure 8.23), Sigmoid PI3 (Figure 8.24), and Sigmoid PI5 (Figure 8.25). As the filters become closer to an inverse filter, and more removed from the matched filter, the main lobe width (at time $=0.45 \mu \mathrm{s}$ ) reduces, and the separation and definition of the secondary peaks improves.

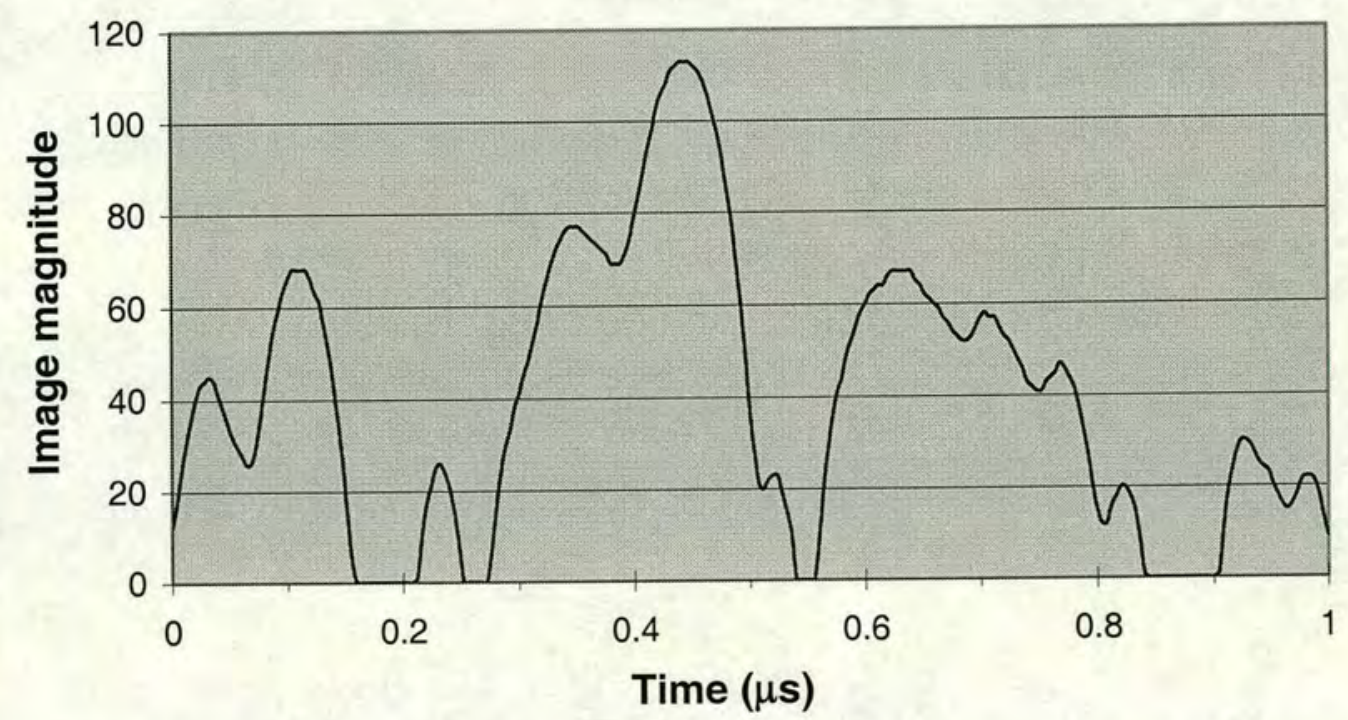

Figure 8.22 Intensity of reconstructed image in region 4 - filtered with filter Sigmoid PII 
As explained earlier, the filter Sigmoid PI1 (see Figure 8.22) is significantly removed from an inverse filter and has only slightly increased the separation between the peaks at time $=0.45 \mu \mathrm{s}$ and time $=0.35 \mu \mathrm{s}$.

The filter Sigmoid PI2 (see Figure 8.23) has significantly improved the separation between the peaks at time $=0.45 \mu \mathrm{s}$ and time $=0.35 \mu \mathrm{s}$; the separate peaks are clearly resolvable.

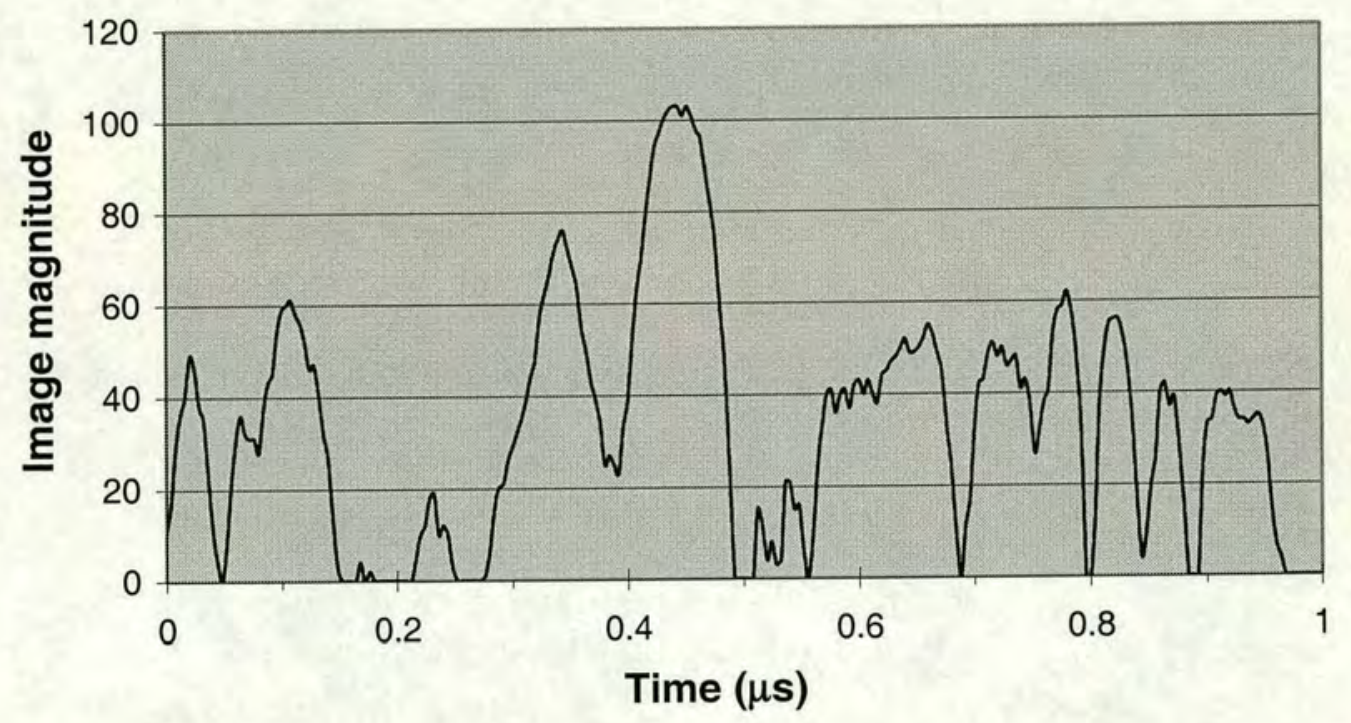

Figure 8.23 Intensity of reconstructed image in region 4 - filtered with filter Sigmoid PI2

The filter Sigmoid PI3 (see Figure 8.24) has improved the separation between the peaks at time $=0.45 \mu$ s and time $=0.35 \mu$ s to a greater degree than the filter Sigmoid PI2. Also, there is some significant resolution of other peaks such as those at time $=$ $0.55 \mu \mathrm{s}$ and time $=0.57 \mu \mathrm{s}$.

The filter Sigmoid PI5 (see Figure 8.25) has improved the separation between the peaks at time $=0.45 \mu \mathrm{s}$ and time $=0.35 \mu \mathrm{s}$ but they are no longer clearly discernible above the apparent noise floor. It is not entirely clear whether the apparent noise is actually noise or signal that has reflected from weakly scattering objects, but it is reasonable to assume that much of it is indeed noise. However the fact that the 
principal peaks are no longer clearly discernible leads to the conclusion that the filter Sigmoid PI5 is too close to an inverse filter for this application.

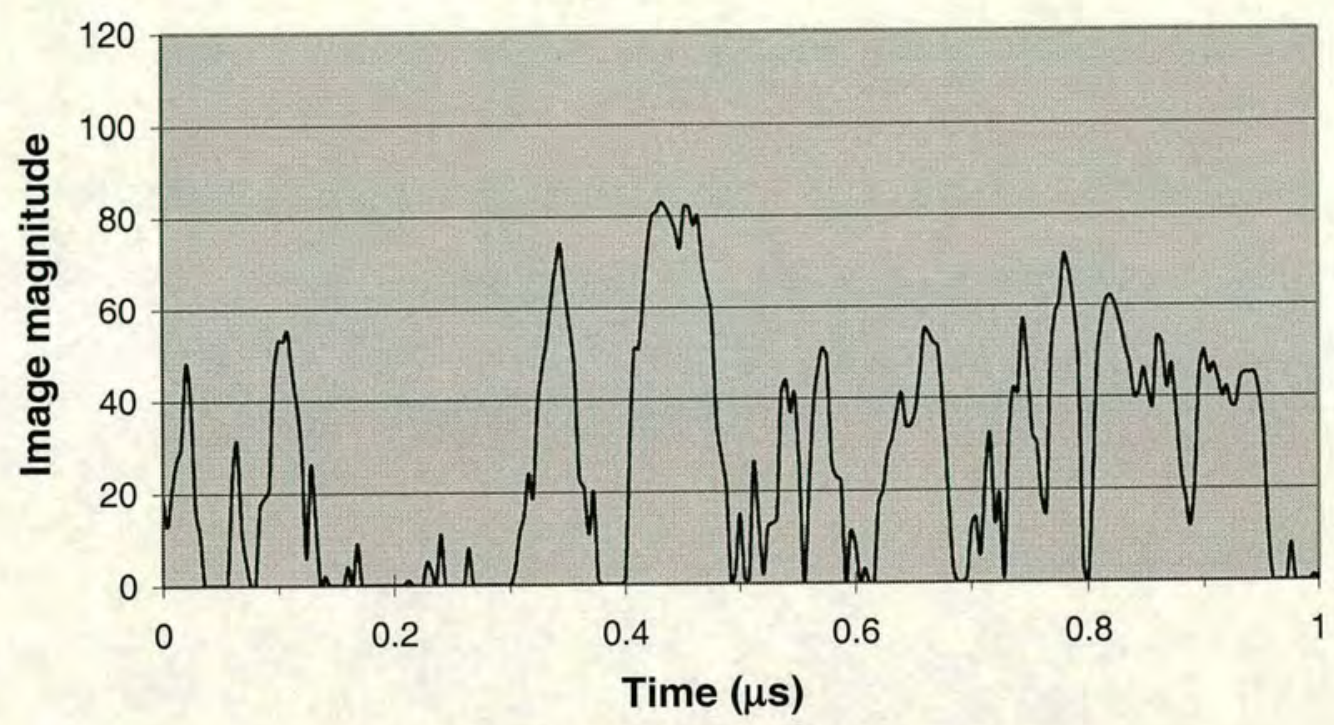

Figure 8.24 Intensity of reconstructed image in region 4-filtered with filter Sigmoid PI3

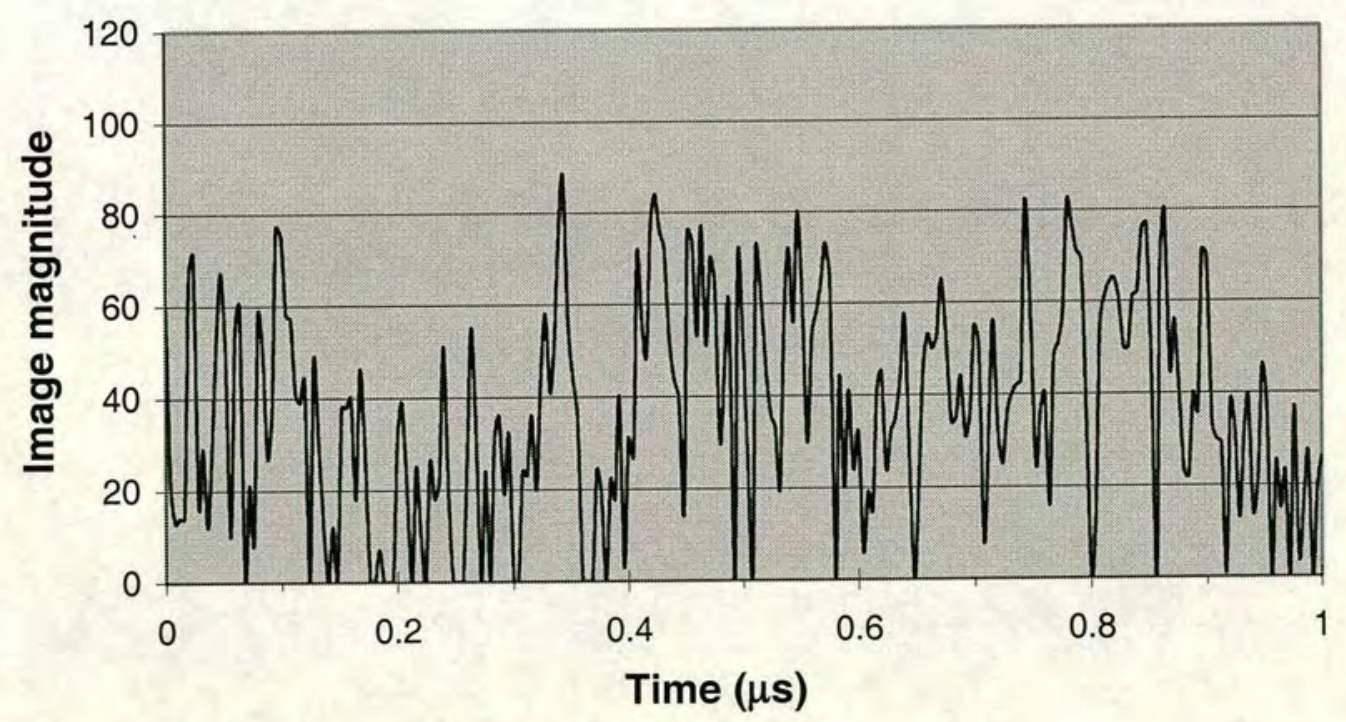

Figure 8.25 Intensity of reconstructed image in region 4 - filtered with filter Sigmoid PI5 


\subsection{Conclusion}

The application of pseudo inverse filters to IVUS images has been demonstrated and proven to enhance the images. The optimal parameters for the pseudo inverse filters have not been found. It is clear that applying a pseudo inverse filter will produce a better IVUS image than either the inverse filter or the matched filter. The matched filter loses too much image resolution and the inverse filter increases the noise too much.

The results of applying pseudo inverse filters to a thin arterial wall have shown that the apparent definition of the edge location is improved - either by enhancing the edge magnitude relative to the noise or by reducing the width of the edge (or both). This is a significant result, allowing greater resolution or noise reduction in 2D Bmode images, and also greater accuracy of surface locations (or existence) in 3D surface rendered images.

The results of applying pseudo inverse filters to a double edge within the image show that pseudo inverse filters are able to resolve multiple edges or surfaces in close proximity. This result proves again that the application of pseudo inverse filters to IVUS ultrasound allows greater resolution (or noise reduction) in 2D B-mode images, and also greater accuracy in establishing the existence (or not) of surfaces for displaying in 3D surface rendered images.

The sigmoid based pseudo inverse filter has yielded images that have a resolution and noise performance that is superior to all of the filters it has been compared with, except for the Wiener MMSE filters. The Wiener MMSE filters and the sigmoid based PI filters have performed at a very similar level, which is a good result for the sigmoid based filters since the Wiener filters are theoretically optimal for minimising the expected MSE for a signal in the presence of AWGN. The AWGN noise assumption does not hold here, and the MSE is not necessarily the best constraint to minimise, but the Wiener filters still provide a good trade off between the noise suppressing matched filter and the resolution enhancing inverse filter. For the 
sigmoid based pseudo inverse filters to perform on a par with the Wiener filters shows that the sigmoid based filters are producing good results. The sigmoid based filters have in some cases produced images that are superior to the images produced by the Wiener filters, such as having less ringdown (Sigmoid PI5 c.f. Wiener filter for $1 \%$ noise), and having greater contrast between double edges (Sigmoid PI3 c.f. Wiener filter for $10 \%$ noise). The optimal filter parameters for both sets of filters (Sigmoid and Wiener) have not been established. 


\section{Chapter 9.}

\section{CONCLUSION}

\subsection{Introduction}

This chapter summarises the main findings of this thesis, and the work reported herein. The aim of the work was to apply an existing filter to a new area, that is a sigmoid based pseudo inverse filter, applied to medical ultrasound images.

In applying the sigmoid based pseudo inverse filter to medical ultrasound images, the design of the filter has been modified. These modifications are original and have not been previously presented.

The following sections of this chapter highlight the main results of this work, and discuss how it might be applied in a clinical setting - including a discussion of the variation in computational complexity between different filters. Ideas for further research are also discussed.

\subsection{The main results of this thesis}

A pseudo inverse filter is proposed for application to medical ultrasound imaging. This filter is based on a sigmoid function. It was initially proposed for application to foetal PCG signals. The definition of the filter design algorithm has been modified, to adapt it for application to the new area - medical ultrasound imaging. The proposed modifications for various situations include the increased attenuation of small Fourier components, and optionally the removal of phase adjustments, and spectral averaging prior to filter design. Further modifications could be easily implemented because of the design approach used. 
The proposed sigmoid based pseudo inverse filter has been applied to ultrasound in three forms: near perfect reflections; phantoms; and rabbit IVUS. The filter has been shown to improve the quality of the images while providing a trade off between contrast resolution and axial resolution.

The proposed filter enhances medical ultrasound images, making the extraction of information easier and more accurate, either for visual inspection in a clinical setting, or in preparing the ultrasound data for surface extraction (for displaying $3 \mathrm{D}$ images).

\subsection{Application in a clinical environment}

These filters have not (yet) been implemented in a clinical setting, but they could be either by the design of an add-in hardware card to perform the filtering in real time, or by post processing (maybe on a PC) to alter the image after it has been captured and displayed in a conventional format. The post processing option will be the easier to implement, but is also less useful for visual inspection - clinicians are accustomed to real time visualisation and this makes their inspections much more efficient. In order to implement these filters in real time, specialised hardware will be required.

For such an application, it is worth noting that for visual inspection in clinical environments, clinicians usually adjust controls such as contrast, brightness, and even TGC while displaying the images - so as to get the best view of the details that they are interested in at the time. Ideally, any implementation of a sigmoid based pseudo inverse filter would allow manual adjustment of the filter parameters (especially the threshold). This would maximise the clinical benefit.

All of the work presented here has been performed as post processing, working on the digitised RF ultrasound signal. The filters presented here have not been implemented in either a clinical or real time environment. 


\subsubsection{Computational complexity}

It is useful to consider the computational complexity of the filters proposed here, and also the complexity of the filters that have been used as comparisons in the results chapters. The length of the filter, or the number of filter taps, will determine how many computations are required to implement the filter for every sample point that is obtained.

In the case of the filters applied to the IVUS data, the system's impulse response was 146 samples long (i.e. after 146 samples it had decayed to a level below the digital quantisation level). The required length any given filter depended on how close the filter was to an inverse filter. The matched filter requires exactly 146 filter taps, while the inverse filter is well approximated by a filter with 438 taps (i.e. three times as long as the impulse response).

For any sigmoid based pseudo inverse filter, the required number of taps is in between the number required for a matched filter, and the number required for an inverse filter (e.g. 230 taps are required for a sigmoid PI3 filter). This number varies slightly when the filter becomes closer to a matched filter or to an inverse filter.

Wiener filters are often designed to be three times as long as the impulse response making them somewhat more computationally expensive than the sigmoid based filters. However the Wiener filters can often be adequately approximated with a shorter tap series - with a length similar to the sigmoid based filters (e.g. for the IVUS system, a Wiener filter designed for $10 \%$ noise can be approximated by a filter with 230 taps - the same number as for the near equivalent sigmoid PI3 filter).

The computational complexity involved in designing the filters is less important than the complexity of implementing the filter - since the design is performed only once while the implementation is performed on a continuous repeating basis. However the design of a sigmoid based pseudo inverse filter requires slightly less computation than the design of an equivalent Wiener filter. Thus the sigmoid based pseudo inverse 
filter approach can be seen to offer a very attractive technique for use in an IVUS system.

\subsubsection{Implementing a practical system}

To implement a filter such as the sigmoid based filter in a clinical environment would require the use of dedicated digital signal processing (DSP) hardware. The DSP unit would have to take as input the digitised data (in RF format), and give the filtered digitised data as output. The DSP unit would be inserted into the ultrasound system immediately after the digitiser, and before envelope detection and image display. In a phased array system, the filter could be implemented either before or after the delay and sum beamforming operation.

For every digitised sample, every tap in the FIR filter must be computed - 230 taps in the case of the IVUS system considered in this thesis. If this were to be done in real time for the IVUS system, then the DSP unit would be required to make 230 multiplication operations every $4 \mathrm{~ns}$ - an overall operating rate of $57.5 \mathrm{GHz}$. This is not currently realisable. However a filtering system may be able to be implemented for other ultrasound imaging systems that have a slower sample rate, although a high degree of parallel processing is probably still required.

A further alternative is to implement the system on a non-real time basis. In this case each frame of data could be stored in temporary memory, and then filtered by a DSP unit while the user waits. The filtered result would then be stored and displayed. In this case the image could not be updated on the screen in real time, however this type of system could still be useful in a clinical setting for "sharpening" a captured image - for the purpose of taking more accurate clinical measurements. 


\subsection{Further work}

One area that could be pursued in further research is to find a means of optimising the filter parameters. However this would probably be different for different applications (e.g. visual analysis and 3D surface extraction). This would probably involve determining the level of noise in the image. However this is difficult since much of the unwanted signal that is received is signal that has been reflected from uninteresting objects and features such as cellular boundaries. For this reason the noise is often referred to as being multiplicative - which is true only if the signal from unwanted objects is classed as noise.

\subsection{Conclusion}

The sigmoid based pseudo inverse filter has been demonstrated as a viable alternative to the Wiener filter, and other pseudo inverse filters, for application to medical ultrasound images. Furthermore, the sigmoid based technique is an easily extendible technique, as demonstrated in the modifications proposed for the phased array phantom processing. In particular this technique is more easily extended and modified than is the case for the Wiener filter.

The modification of this filter, and its application to a new area (medical ultrasound imaging) constitutes a new and original contribution to knowledge. 


\section{Appendix A. BILINEAR INTERPOLATION}

\section{A.1. The problem}

During the scan conversion process, it is necessary to convert the ultrasound data from a polar (or cylindrical) co-ordinate system to a rectangular co-ordinate system.

The ultrasound data is measured as a series of 1D scans, which are combined to form $2 \mathrm{D}$ scans. The $1 \mathrm{D}$ scans are sampled in time, and are geometrically arranged on a polar co-ordinate system as shown in Figure A.1. The samples are regularly spaced on polar axes (radius $r$ and angle $\theta$ ).
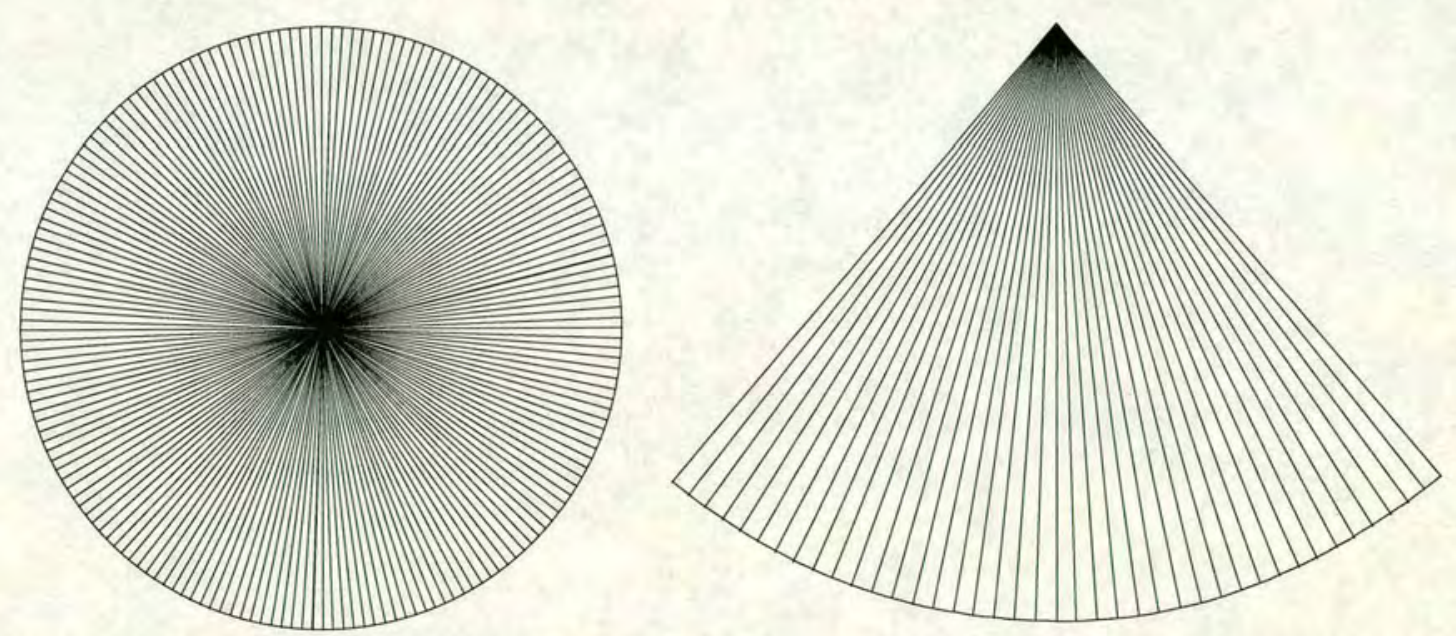

Figure A.1 Possible arrangements of $1 D$ scanlines on a polar grid

In order to reconstruct a $2 \mathrm{D}$ image on rectangular co-ordinates from the polar coordinate based data, a bilinear interpolation algorithm is used. This problem is illustrated for a polar grid in Figure A.2. The separation of samples (or scanlines) in angle is defined as $\Delta \theta$, and the separation of samples in radial distance (originally in time) is defined as $\Delta \mathrm{r}$. 


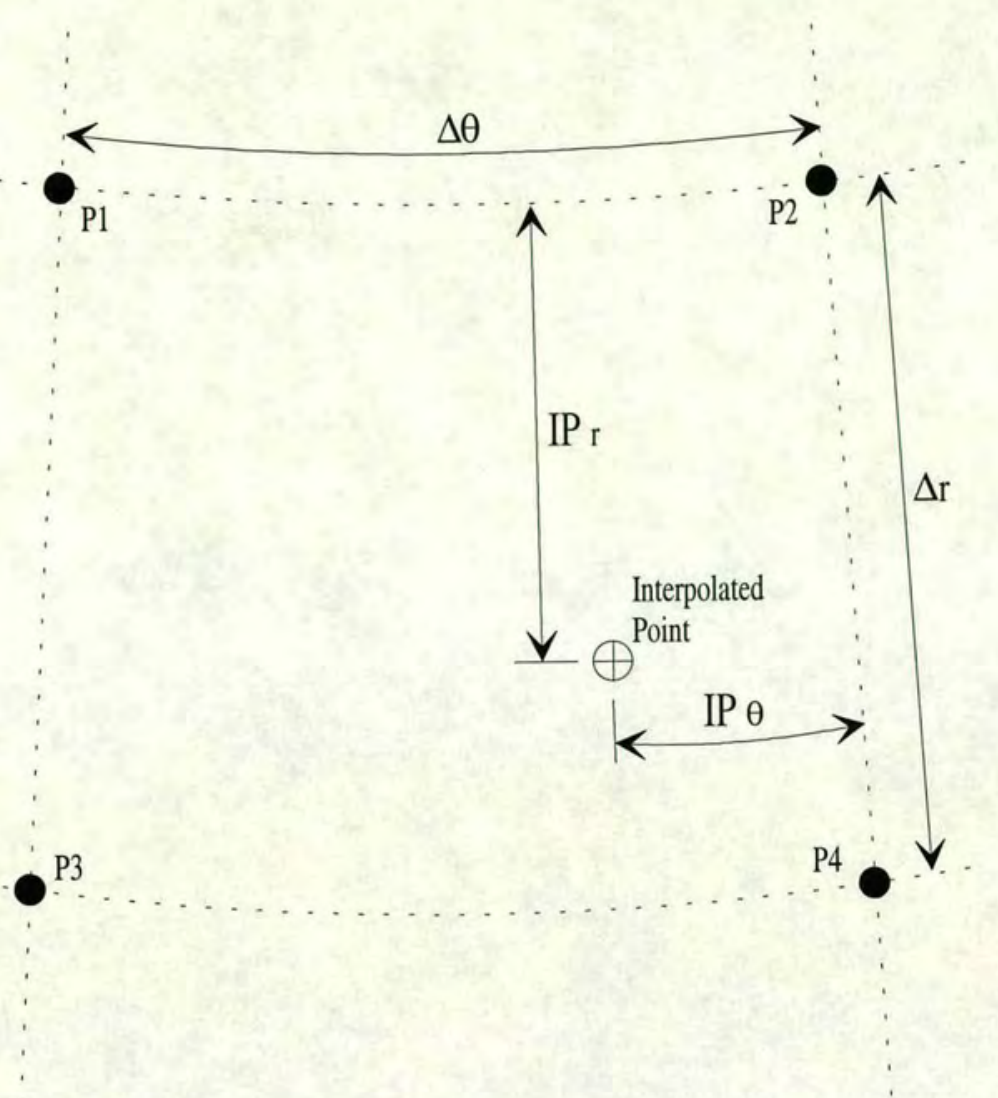

Figure A.2 Bilinear interpolation problem on a polar grid

\section{A.2. Definition of the bilinear interpolation}

In Figure A.2 the point for which we wish to perform the interpolation, is labelled as the "Interpolated Point". The four points that surround the interpolated point - for which samples have been obtained - are labelled P1, P2, P3, and P4.

The radial distance to the interpolated point is a distance $\mathrm{IP}_{\mathrm{r}}$ greater than the radial distance to the points $\mathrm{P} 1$ and $\mathrm{P} 2$. The angular offset to the interpolated point is $\mathrm{IP}_{\theta}$ greater than the angular offset to the points P2 and P4. The angular offset is defined here to be increasing with clockwise movement.

Note that the units of $\mathrm{IP}_{\mathrm{r}}, \mathrm{IP}_{\theta}, \Delta \mathrm{r}$, and $\Delta \theta$, are not important - it is only the ratios that matter. 
The bilinear interpolation defines the value of the interpolated point according to equation A.5. The value of each of the four surrounding points contributes to the value of the interpolated point according to the location of the interpolated point.

$$
\begin{aligned}
& \text { Value }(I P)= \frac{I P_{\theta}}{\Delta \theta} \cdot\left(1-\frac{I P_{r}}{\Delta r}\right) \cdot \operatorname{Value}(P 1)+\left(1-\frac{I P_{\theta}}{\Delta \theta}\right) \cdot\left(1-\frac{I P_{r}}{\Delta r}\right) \cdot \operatorname{Value}(P 2)+ \\
& \frac{I P_{\theta}}{\Delta \theta} \cdot \frac{I P_{r}}{\Delta r} \cdot \operatorname{Value}(P 3)+\left(1-\frac{I P_{\theta}}{\Delta \theta}\right) \cdot \frac{I P_{r}}{\Delta r} \cdot \operatorname{Value}(P 4)
\end{aligned}
$$

\section{A.3. An alternative approach}

In order to aid the understanding of equation A.5, it may be useful to consider the bilinear interpolation defined in equation A.5 as a two stage linear interpolation. Firstly, in the angular direction, the percentage of $\left(\operatorname{IP}_{\theta} \div \Delta \theta\right)$ is used to interpolate between $\mathrm{P} 1$ and $\mathrm{P} 2$, and (separately) between $\mathrm{P} 3$ and P4, see equations A.6 and A.7. This yields two intermediate points $\mathrm{P}_{1-2}$ and $\mathrm{P}_{3-4}$. Then secondly, in the radial direction, the percentage $\left(\mathrm{IP}_{\mathrm{r}} \div \Delta \mathrm{r}\right)$ is used to interpolate between the two intermediate points $\mathrm{P}_{1-2}$ and $\mathrm{P}_{3-4}$, see equation $\mathrm{A} .8$.

$\operatorname{Value}\left(P_{1-2}\right)=\left(\frac{I P_{\theta}}{\Delta \theta}\right) \cdot \operatorname{Value}(P 1)+\left(1-\frac{I P_{\theta}}{\Delta \theta}\right) \cdot \operatorname{Value}(P 2)$

$\operatorname{Value}\left(P_{3-4}\right)=\left(\frac{I P_{\theta}}{\Delta \theta}\right) \cdot \operatorname{Value}(P 3)+\left(1-\frac{I P_{\theta}}{\Delta \theta}\right) \cdot \operatorname{Value}(P 4)$

$\operatorname{Value}(I P)=\left(1-\frac{I P_{r}}{\Delta r}\right) \frac{I P_{r}}{\Delta r} \cdot \operatorname{Value}\left(P_{1-2}\right)+\left(\frac{I P_{r}}{\Delta r}\right) \cdot \operatorname{Value}\left(P_{3-4}\right)$

Combining equations A.6, A.7, and A.8 will yield equation A.5. 


\section{Appendix B.}

\section{ENCLOSED SOFTWARE}

The work in this thesis was carried out using a variety of software. All software for implementing the algorithms presented in this thesis was written by myself and is described in this and subsequent appendices.

The software applications (or programs) are written in two languages - Delphi (i.e. Pascal) and IDL. All programs including source code are reproduced on the compact disc attached to this thesis. The CD is formatted to be read by an IBM compatible PC.

The software programs are:

- a program for filter building and manipulation (the primary application). This program is called "Phaser.exe" and is described in Appendix C;

- a program for bitmap colour transforms. This program is called "Color256.exe" and is described in Appendix D;

- a program for extracting numerical ultrasound intensity data from bitmap files. This program is called "Bmp2Text.exe" and is described in Appendix E;

- programs for scan converting images (written in IDL). There are several scan conversion programs and they are all described in Appendix F.

In the next appendices are instructions describing the basic operation of these programs. These are not intended as full user guides, but as a source of information on how to get started with each piece of software, what some of its capabilities are, and how to do some of the more commonly performed tasks.

The first three programs all require Windows95 or Windows NT4 in order to run. 


\section{Appendix C.}

\section{SOFTWARE FOR \\ IVUS PROCESSING}

This is the principal program used within this thesis, and it implements all the algorithms presented except for the scan conversion and Hilbert transform algorithms.

This program is approximately 11,400 lines of Pascal code - according to the compiler generated line count. This is in addition to the RAD design of the user interface which is excluded from the compiler generated line count, and the visual component library (VCL) that is supplied with Delphi 3. This represents many months of programming effort.

\section{C.1. Main function of program}

The program is designed to be able to construct any of the filters used or presented in this thesis, and to apply those filters to ultrasound data. Any filter or ultrasound data can be graphed in the time domain or frequency domain.

At any stage of filter construction, the data can be saved to a file and reloaded at a later time. All data is saved as truecolour (24-bit) bitmap files. All data loaded is also expected to be truecolour bitmaps. For using 256 colour greyscale bitmaps, see Appendix $\mathrm{D}$ for instructions on converting the file to a truecolour bitmap.

\section{C.2. How to make and apply a sigmoid based filter}

In order to construct and apply a filter you need to have the following files: a near perfect reflection image, and an image that needs to be filtered. 


\section{C.2.1. Constructing the filter}

To construct the filter you should use the menu command "File | Open", which will allow you to choose a file from disk - choose the bitmap file that contains the near perfect reflection image.

You may at this point choose to utilise only one scanline from your image (this is generally wise with single element transducers but unwise with phased array transducers). If you choose this, then select a suitable line (i.e. a line with a large but not clipped reflection). You can find a suitable line by graphing each line of the image (by repeatedly using the menu option "Graph | Graph next line"). Having selected an appropriate line, choose the menu option "Settings | Options", choose the "greyscale" tab, and set the "ActiveRow" to be the chosen row number. Finally select the menu option "Averaging | Replicate current row". The bitmap will now have every row or scanline being identical to your chosen row or scanline. The bitmap could be saved at this point using the menu option "File | Save As...".

\section{Windowing the impulse response}

If the scanlines contain multiple reflections then you might choose to clean up the signal by applying a window function over the perfect reflections. Choose the menu option "Settings | Options", choose the "Windowing" tab, and select your window limits (in units of pixels along the $\mathrm{x}$-axis of the image) and the window shape. Then choose the menu option "Filtering | Window function". Again, the bitmap could be saved at this point.

\section{Fourier transforming}

The sigmoid based filters are constructed in the frequency domain. Choose menu option "FFT $\mid$ FFT $\rightarrow$ Bmp". This will apply an FFT to each row of the image. It is a good idea to save the image to a disk file at this point with a $f f t$ extension. 
You may choose to remove the phase correction (or phase alignment) property of the filter. In order to do this, you should select the menu option "FFT | Linearize the Phase $\rightarrow$ Bmp". You may also choose to average the spectrums (this is a good idea only if you have "linearized the phase"). To do this, you must select firstly the menu option "Averaging | Average all rows", and then the menu option "Averaging | Replicate current row". (Note, the active row on the "Greyscale" tab of the "Settings | Options" dialog must not be set to the either of the two highest rows in the image for this particular step).

\section{Sigmoid based pseudo inversion}

Choose menu option "Settings | Options", and choose the "Pseudo Inverse" tab to select the parameters of the sigmoid based pseudo inverse equation (Gain $G$, Threshold $T$, offset $d$ ). You must also specify the maximum value in the image (i.e. the image of the FFT). If you don't know this value, you can get it after performing a (trial run) pseudo inversion, then enter the maximum value here, and repeat the pseudo inversion (see below).

The tickbox "Force zero gain at zero signal" will choose between the original Sigmoid equation (equation 4.1, for this option leave the tickbox empty) and my modified equation (equation 4.4 , for this option leave the tickbox ticked).

At the bottom of the "Pseudo Inverse" tab are several quick buttons which will set up predefined parameter sets - including the filters Sigmoid PI1, PI2, PI3 and PI5 presented in this thesis.

Click OK to save your parameters and exit the options dialog.

To perform the pseudo inversion, choose the menu option "Pseudo Inverse / Make filter by amplitude". This will perform the sigmoid based pseudo inverse function. Note that when this operation finishes, you will be told what the maximum value in the image was. You may choose to use this information in the filter setup in the options dialog. 


\section{Inverse Fourier transforming}

In order to make the filter taps, the inverted FFT must be returned to the time domain. Do this by selecting the menu option "FFT $\mid$ IFFT $\rightarrow$ Color BMP".

You now have your filter taps. However you may wish to truncate the filter taps by applying a window function to them (see "Settings | Options", "Windowing" tab and menu option "Filtering | Window function"). Note that the filter taps will be positioned in the bitmap at the position "ImageWidth - position of perfect reflection".

You must save your filter taps in order to use them (menu option "File | Save As..."). The file extension . pi can be used for pseudo inverse filter files.

\section{C.2.2. Applying the filter}

In order to apply the filter, the filter must be saved to a file. You must also have a truecolour bitmap of the image you want to be filtered. Load the image to be filtered by choosing the menu option "File | Open".

Ensure that the settings in Windowing tab (of the "Settings | Options" dialog) specify that the low cutoff and high cutoff are set to values which encompass all of the original perfect reflection. These parameters are used to truncate the filter taps (to save computation time). The filter taps that are used are those points in the filter bitmap rows that fall between the columns 'ImageWidth - High cutoff' and 'ImageWidth - Low cutoff'. Where ImageWidth is the width of the filter bitmap (determined automatically by the program).

Perform the filter operation by selecting the menu option "Filtering | Filter Row by Row from Filter BMP | with output enlarged" (or use the output reduced option if you find the output values are so large that the bitmap pixels saturate - they saturate at $2^{23}$ or 8.4 million). You can confirm the filter output by graphing the resulting rows or scanlines - choose the menu option "Graph | Raw data". 


\section{C.3. Other program functions}

\section{C.3.1. Creating a Wiener filter}

You can create a Wiener filter by using the menu option "Wiener | Create Wiener Filter". The options for the Wiener filter are set by selecting the menu option "Settings | Options", and choosing the "Wiener" tab. The Wiener filter (as a bitmap image) that is produced by this operation is a time reversal of what the filter should be. In order to fix this, the filter needs to be saved (as a bitmap) and reversed left to right. Paint Shop Pro can do this using the menu option "Image | Mirror". Alternatively Microsoft Paint for Windows95 can do it using the menu option "Image | Flip/Rotate" and choosing "flip horizontal".

\section{C.3.2. Creating a Square root filter}

This is the same as creating a sigmoid based inversion filter, except that the step for the sigmoid based pseudo inversion should be replaced by selecting the menu option "Rooter|Make square root filter"

\section{C.3.3. Creating a phase correction filter}

This is the same as creating a sigmoid based inversion filter, except that the step for the sigmoid based pseudo inversion should be preceded by selecting the menu option "FFT | Set Amplitude to Unity"

\section{C.3.4. Creating a matched filter}

To create a matched filter, use Paint Shop Pro or Microsoft Paint, and reverse (left to right) the near perfect reflection bitmap. You may still choose to clean up the image 
by applying a window function to the bitmap. See the section Windowing the impulse response in section C.2.1.

\section{C.3.5. Creating an inverse filter}

This is the same as creating a sigmoid based pseudo inverse filter, except that inside the "Settings | Options" dialog, on the "Pseudo inverse" tab you should press the quick button labelled "True Inverse". 


\section{Appendix D. SOFTWARE FOR BITMAP COLOUR TRANSFORMS}

This software is useful for converting bitmaps into truecolour. The data that come from some transducers is 8 bit. Attaching a bitmap header can make this an 8-bit greyscale image. This program will convert that to a 24-bit greyscale image, or to a 24-bit red image. The motivation for a "red" image is that the filter generation program in Appendix $\mathrm{C}$ will be able to use smaller numbers as a result of this transformation. Consequently the bitmap pixels will saturate less easily.

This program is approximately 2,500 lines of Pascal code -in addition to Delphi's VCL.

\section{D.1. Operating the program}

You can load any bitmap file using the menu option "File | Open". Then you can save the file in an alternative format using the menu option "File | Save As...". When saving you have a choice of three formats:

- greyscale (8-bit);

- truecolour (24-bit greyscale);

- truecolour (24-bit) red.

It is not possible to convert directly from a truecolour grey image to a 24-bit red image or vice versa.

The IVUS processing program in Appendix C can process bitmaps saved in either of the two available truecolour formats. 


\section{Appendix E. SOFTWARE FOR EXTRACTING DATA FROM BITMAPS}

This program is useful for extracting the ultrasound data from a bitmap file, so that it can be plotted on a graph. This program will save a row (or multiple rows) from a bitmap as a text file, which can then be imported into MS Excel, (and most likely into any major spreadsheet or graphing package). This program is approximately 2,500 lines of Pascal code - in addition to Delphi's VCL.

\section{E.1. Operating the program}

Firstly, select the menu option called "Options!". This allows you to specify which row of the bitmap you will save, and how many rows you will save. It also allows the delimiting character to be chosen (using a tab character works well for MS Excel).

Next you must open a bitmap file using the menu option "File | Open". You can then save the desired row from the bitmap, as delimited text by choosing the menu option "File | Save As...".

\section{E.2. The output format}

The saved file will be able to be imported into MS Excel as a text file. It contains each saved row of the bitmap as a column of numbers. Each number represents the value of a corresponding pixel in the bitmap. Within each bitmap row (or text file column), the leftmost pixel is written at the top of the column in the text file, and the rightmost pixel is at the bottom of the column in the text file. There will be one column for every bitmap row saved, plus an index column. Successive columns are separated by the delimiting character chosen in the "Options!" dialog box. 


\section{Appendix F.}

IDL SOFTWARE FOR SCAN CONVERSION

There are three programs used within this thesis for processing ultrasound images with the application IDL (Integrated Development Language). They cannot be run without an installation of IDL (with a valid user license). These programs perform scan conversion for ultrasound images, both IVUS and phantoms. A separate program also will perform only the Hilbert transform and log transform. Each program is around 380 lines of code and is described in the following sections.

\section{F.1. The different IDL programs}

\section{F.1.1. IVUSScanConvert.pro}

This program will perform a scan conversion on a bitmap, and will use a geometric correction that is consistent with the geometry of the IVUS data collection system used in this thesis. Principally, this means that the data is assumed to cover an acquisition angle of $360^{\circ}$. The program can be easily altered to use various sizes of output image, and also different dynamic ranges in the output image. The default is for the output images to be 512 x 512 pixels, and have a dynamic range of $50 \mathrm{~dB}$. To change this default you must alter the program code.

\section{F.1.2. PhantomScanConvert.pro}

This program will perform a scan conversion on a bitmap, and will use a geometric correction that is consistent with the geometry of the phased array data collection system used in this thesis for scanning the phantom. Principally, this means that the 
data is assumed to cover an acquisition angle of $77^{\circ}$. Otherwise the program is identical to IVUSScanConvert.pro.

\section{F.1.3. HilbertTransform.pro}

This program makes no assumptions about the geometry format of the data. Each row in the bitmap is treated independently. Each row is Hilbert transformed, and log transformed to a dynamic range of $50 \mathrm{~dB}$. The output image is always the same size as the input image. The program can be easily altered to use different dynamic ranges in the output image. The default is for the output image to have a dynamic range of $50 \mathrm{~dB}$.

\section{F.2. Characteristics shared by the IDL programs}

The three IDL programs described above all share some characteristics (as well as much code). These characteristics are:

6. The input image to all three programs must be an uncompressed bitmap. The bitmap can be an 8 bit greyscale image, or it can be a truecolor bitmap such as those produced by other programs used in this thesis for IVUS processing (Appendix C) and for Bitmap colour transforms (Appendix D).

7. The output image is always stored in a TIFF file format. This can be easily converted to a bitmap using the shareware software Paint Shop Pro (PSP).

8. The resulting bitmap (after conversion with PSP) will have been flipped upside down. PSP can flip the image back again if you choose.

9. The program will process multiple files at once. Any bitmap file with a.$b m p$ extension in the directory (chosen by the user at runtime) will be processed (or converted) providing there is not a file in that directory with the same name and a .tif extension. 


\section{Appendix G. OTHER ENCLOSED FILES}

\section{G.1. Applications}

Because of the reference made within this thesis, an evaluation version of the program Paint Shop Pro (PSP) is included on the accompanying CD. This program is shareware, produced by the company JASC. Inc. and is reproduced on this CD with the permission of JASC. Inc.

\section{G.2. Ultrasound data samples}

\section{G.2.1. Phantom samples}

The accompanying $\mathrm{CD}$ includes two full image sets of the phantom, plus some images of a near perfect reflector obtained with the same transducer. All of this data is on the accompanying CD in the directory "Phased Array Phantom Data".

The two image sets of the phantom each cover $180^{\circ}$ - which covers the whole target volume. These images are in the subdirectories "Block1" and "Block2" respectively. The perfect reflection images are in the subdirectory "Perfect Reflections".

In order to aid the readers understanding of the geometry involved in the phantom, a video file is also included in the subdirectory "Block1\Video". This file presents the successive frames of the Block1 image set, in the sequence that they were acquired (i.e. as the transducer was rotated through $180^{\circ}$ ). 


\section{G.2.2. IVUS samples}

The accompanying CD includes one full set of images from an IVUS pullback, plus some images of a near perfect reflector obtained with the same transducer. All of this data is on the accompanying CD in the directory "Intravascular Ultrasound Data".

The IVUS pullback data is in the subdirectory "Pullback A of Rabbit 1". The perfect reflection images are in the subdirectory "Perfect Reflections".

In order to aid the readers understanding of the geometry involved in the IVUS data and the imaged aorta, a video file is also included in the subdirectory "Pullback A of Rabbit 1IVideos". This file presents the successive frames of the "Pullback A" image set, in the sequence that they were acquired (i.e. as the transducer was pulled backwards through the aorta). There are multiple versions of this file, with the filename indicating the type of filter that was applied to the images. The original data is in the file called "Unfiltered.avi". 


\section{Appendix H. ORIGINAL PUblications}

Parts of the work and results presented in this thesis have been previously published.

The publication, which is included here is:

- Young, W. F. and McLaughlin, S. "Pseudo Inverse Filter Design for improving the Axial Resolution of Ultrasound Images". This was published in the IEEE Tencon Conference on Speech and Image Technologies for Computing and Telecommunications, 1997, Vol. 2, p703-6. ISBN 0780343654. 


\title{
PSEUDO-INVERSE FILTER DESIGN FOR IMPROVING THE AXIAL RESOLUTION OF ULTRASOUND IMAGES
}

\author{
Warren F. Young and Steve McLaughlin
}

\section{Department of Electrical Engineering}

The University of Edinburgh, King's Buildings, Mayfield Road, Edinburgh EH9 3JL Tel: 01316505577 Fax: 01316506554 Email: wfy@ee.ed.ac.uk

\begin{abstract}
he resolution of medical ultrasound systems can e improved using filtering techniques. We present filter for improving the axial resolution that pproximates an inverse filter but which is less ensitive to noise than a standard inverse filter naking it suitable for use with ultrasound. This ilter produces a superior image resolution to that rained by matched filtering, and also shows dvantages over a Wiener optimal filter, and other ilters presented in literature.
\end{abstract}

\section{INTRODUCTION}

$\mathrm{n}$ this paper we present a technique for designing a ilter that improves the resolution of an ultrasound ystem, by approximating an inverse filter. We how that the results from such a filter provide uperior resolution to that from a matched filter.

\section{.1 The Problem}

Accurate measurements and the precise location of listinguishing features in medical ultrasound maging are important for clinical decision making 1] (e.g. foetal dimensions for measuring growth; he thickness of plaques in intravascular ultrasound IVUS]; and carotid thickness measurements).

When the ultrasound transducer is 'hit', it emits a oroadband impulse, with a centre frequency of ypically 2 to $30 \mathrm{MHz}$, and lasting several cycles see Figure 1. The duration of this pulse limits the axial resolution of the image, reducing the accuracy of clinical measurements made from the resulting mages - whether the image is presented in $2 \mathrm{D}$ or 3D.

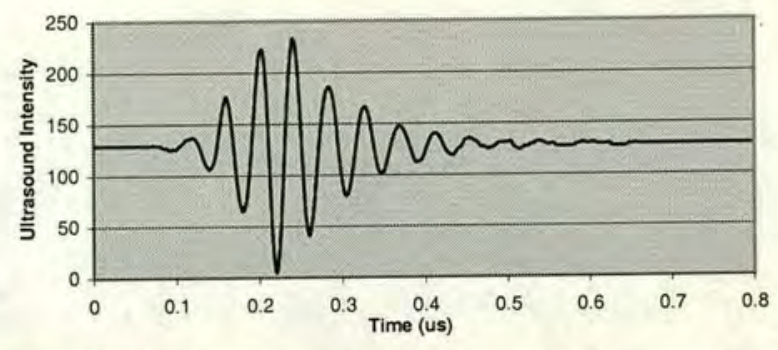

Figure 1 Typical pulse from a single element IVUS transducer.

\subsection{Other Filtering Techniques}

Conventional filtering techniques for spiking, deconvolution or inverse filtering can reduce the effective impulse duration in systems that have high signal to noise ratios. However ultrasound systems have poor signal to noise ratios. A pseudoinverse filter can overcome these limitations, and offer a significant improvement to the axial resolution.

Other researchers have presented techniques for achieving various forms of pseudo-inverse filters.[2][3]

\section{PSEUDO-INVERSE FILTER DESIGN}

We present a sigmoid based pseudo-inverse filter, tailored to the characteristics of the ultrasound transducer. This filter is designed to be tolerant of noise, while still performing a pseudo frequency inversion. This filter was first presented in the analysis of foetal heart ECG[4][5].

\subsection{Design algorithm}

We consider the pulse reflected from a near perfect plane reflector to be a near perfect estimate of the system impulse response.

The filter is constructed in the frequency domain.

The Fourier components of the system impulse 
esponse are normalised and then pseudo-inversed ccording to equation 1 .

$$
I .(\omega)=\frac{\operatorname{Arg}\left(F^{*}(\omega)\right) \cdot \operatorname{Sig}(G \cdot(|F(\omega)|-T))}{|F(\omega)|+d}
$$

Where $\operatorname{Sig}(x)$ is a sigmoid function, with values anging between 0.0 and 1.0 ; defined as:

$$
\operatorname{sig}(x)=\frac{1}{1+e^{-x}}
$$

n equation $1, G$ is a gain factor (typically 10 to 00 ), $\mathrm{T}$ is a threshold value, typically $1 \%$ to $10 \%$ of he normalised $\mathrm{F}(\omega)$ components, and $\mathrm{d}$ is a small tabilising factor (typically 0.01 to 0.05 ). The igmoid function provides a smooth transition etween an inverse filter (for large Fourier components) and a matched filter (for very small Fourier components)

The filter taps are formed by subsequent conversion back into the time domain by using an nverse Fourier transform.

\subsubsection{Selecting parameter values}

Varying the design parameters (particularly the hreshold and the gain) yields differing pseudonverse functions as shown in Figure 2.
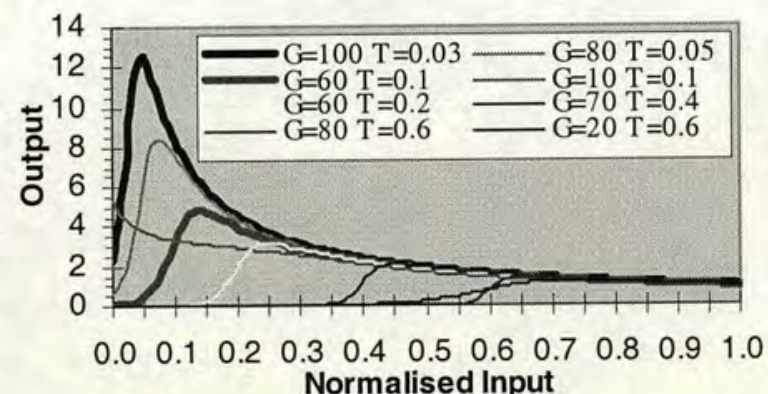

Figure 2 Sigmoid based pseudo inverse functions

We found good results were obtained with design parameters of $50 \leq \mathrm{G} \leq 150, \quad 0.01 \leq \mathrm{T} \leq 0.1$, and $0.01 \leq \mathrm{d}<0.05$. These ranges result in filters that are sufficiently insensitive to noise (for the IVUS data studied here) while giving good image enhancement. We present results for two sets of design parameters. 1) $\mathrm{G}=100, \mathrm{~T}=0.03, \mathrm{~d}=0.02$, and 2) $\mathrm{G}=60, \mathrm{~T}=0.1, \mathrm{~d}=0.05$ (see thicker lines in Figure 2).

\subsection{Example Filter Design}

This filter is designed for an IVUS system - its impulse response is shown in Figure 1, and its power spectrum is shown in Figure 3. We use the design parameters given above.

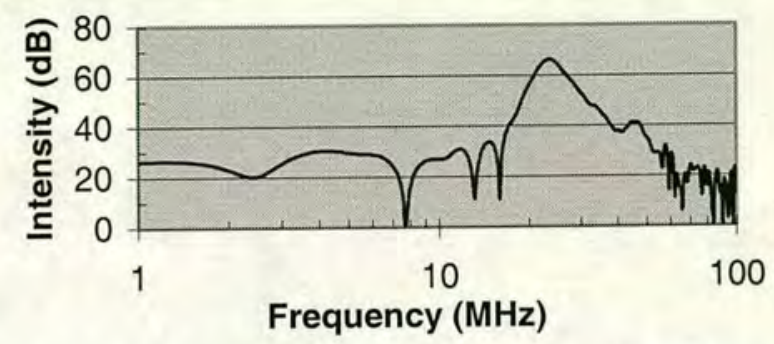

Figure 3 Power spectrum of IVUS system impulse response.

Each Fourier component is normalised, and then sigmoid pseudo-inversed using equation 1 . The gain function of the two resulting filters is shown in Figure 4. Note that the gain function is close to an inverse within the passband, and is close to a level shifted matched filter outside the passband.

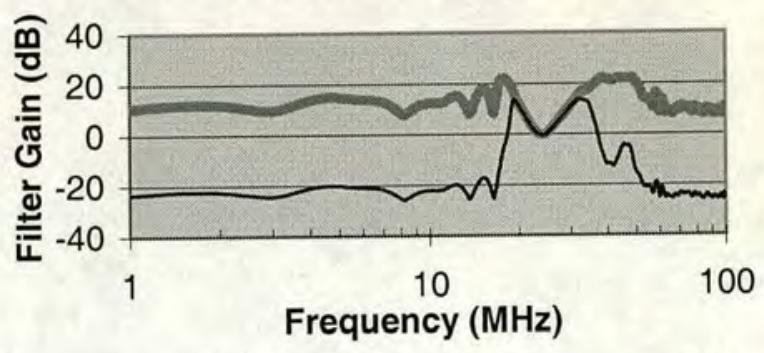

Figure 4 Transfer function for two sigmoid pseudo-inverse filters.

Multiplying the system impulse response and the filter response gives the power spectrum shown in Figure 5.

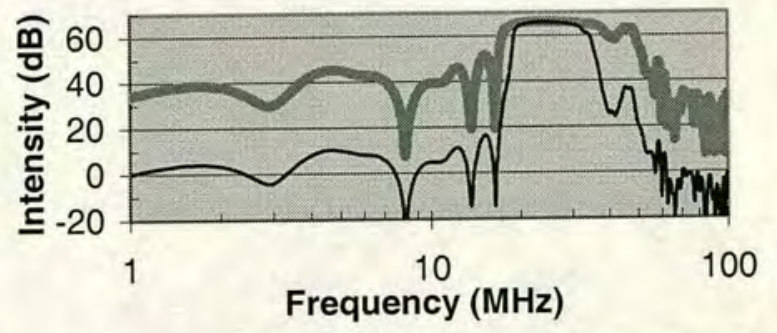

Figure 5 Power spectra after applying two sigmoid pseudoinverse filters to the system impulse response. 


\section{RESULTS OF PSEUDO-INVERSE FILTERS ON ULTRASOUND NEAR PERFECT REFLECTIONS}

igure 6 shows the IVUS system impulse response f Figure 1 after being filtered with the first xample filter designed in section 2.2. The eduction in the main pulse width is significant.

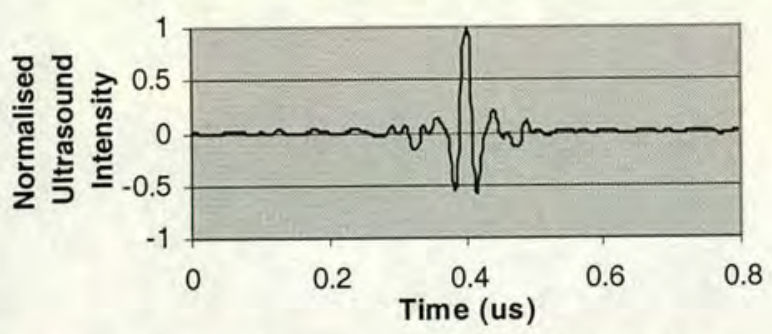

igure 6 IVUS system impulse response filtered with sigmoid seudo-inverse filter $(G=100, T=0.03, d=0.02)$

\section{RESULTS OF PSEUDO-INVERSE FILTERS ON ULTRASOUND IMAGES}

The Intravascular ultrasound (IVUS) data resented here is from the same single element ransducer used in section 2. 240 radial scan lines are used to make up the image (the scan lines being separated in angle by $1.5^{\circ}$ ). The data is presented nere in a scan-converted format. The scan conversion involved a Hilbert transform (for envelope detection), then interpolation, a log ransform and geometric correction to make the inal image. All images presented have a dynamic ange of $50 \mathrm{~dB}$.

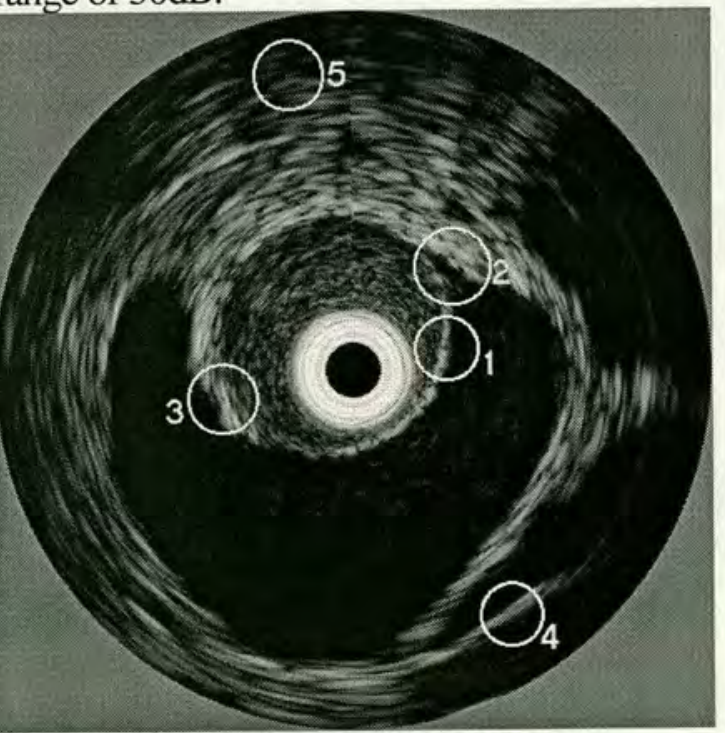

Figure 7 IVUS image from a rabbit - unfiltered.
The five marked regions of interest are:

1. A thin vessel wall whose precise location is clinically useful information.

2. Two transition regions in close proximity. The precise distance between the boundaries is clinically useful information.

3. A thick area of echogenic material, whose width is clinically useful information.

4. A double edge - although the second (outer) edge is not visible in this image. In this instance the outer edge is not clinically useful but serves as an example of resolving multiple edges in close proximity.

5. An edge, obscured with "speckle". The edge in this instance is not clinically useful, but serves as a test for edge extraction from within "noise".

\subsection{The Filters used}

The filters are applied only in the axial dimension, prior to scan conversion.

The filters compared here are:

1. A sigmoid based pseudo-inverse filter $(\mathrm{G}=100$, $\mathrm{T}=0.03, \mathrm{~d}=0.02$ ), as designed in section 2 .

2. A matched filter,

Other filters not compared are a Wiener optimal filter, a filter for aperture based phase correction only, a filter based on an SVD based approach [2], and a filter utilising square root frequency based pseudo-inversion with aperture based phase correction.[6]

\subsection{Comparison with unfiltered image}

In the five areas of interest above it is clear that a superior resolution has been obtained.

The width of the edge in area 1 is narrower, allowing more accurate estimation of its precise location. (This is useful for clinical diagnosis, and also for edge detection algorithms used in automatic area/volume estimations and in surface based three dimensional reconstructions).

In areas 2 and 3 the edges have become slightly more defined. 


\section{Appendix I. SIGMOID BASED FILTER}

The sigmoid based pseudo inverse filter was first presented by Basil and Dripps. The following pages (all of Appendix I) are reproduced from H. Basil's Ph.D. thesis [80], for convenient access for the reader.

These pages describe the motivation for, and the thinking behind the original proposal of the sigmoid based pseudo inverse filter.

The pages reproduced here have been renumbered here for continuity within this thesis - the pages were originally numbered as pages 62 to 76 inclusive in H. Basil's thesis. 


\subsubsection{Pseudo-Inverse Filter}

An original contribution to time domain beat-to-beat estimators, namely the PseudoInverse Filter (PIF), is detailed in this section. The advocated new algorithm derives the weights of a partially equalising filter which improves the ability to detect HPW occurrences. The pseudo-inverse filter algorithm is based on the following model: 
The assumption is that oscillatory waveforms observed at the maternal abdomen, identified as the first and second HPW's, emanate from the fetal heart as a train of unipolar impulses. In travelling through the fetal and maternal abdominal structures (organs) to the abdominal surface and through the transducer, these impulses suffer attenuation and dispersion (Fig. 3.13). This implies that an observed HPW pulse is the impulse response of this described model.

Based on such a model, the transfer function between the impulse train source and the abdominal surface has a non-flat amplitude and a non-linear phase response.

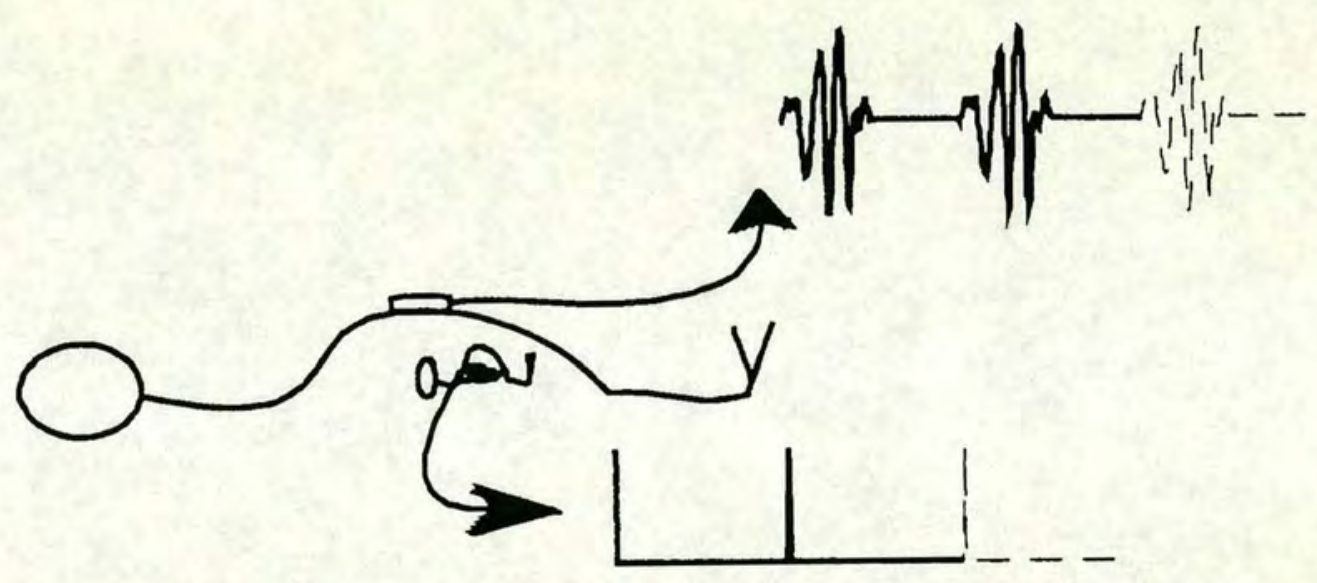

Figure 3.13 Illustration of both patient and fetus depicting the adopted model for HPW generation. Impulses initiated at the fetal heart (lower trace) are dispersed by the path to the transducer (upper trace).

Figure 3.14 shows a typical sampled first HPW reflecting the impulse response of the adopted model. While the second HPW is subjected to the same spatial filtering described above (through fetal and maternal organs), the underlying physical event has a different constitution. In consequence, both its time and spectral shapes are dissimilar to that of the first HPW (see chapter 2). Therefore a similar model but with 
different dispersion characteristics must be assumed for the second HPW. The approach described here concentrates on extracting first HPW impulses from the maternal abdominal signals. A similar approach can be adopted for the second HPW extraction.

The advocated pseudo-inverse filter approach attempts to equalise both amplitude and phase responses of the first HPW. Therefore the reconstructed impulse train should, except for a processing delay imposed by the filter, pin-point the onset of systole events. While PIF processing is performed in the time domain, the derivation of the filter coefficients is achieved using a spectral estimate of the first HPW.

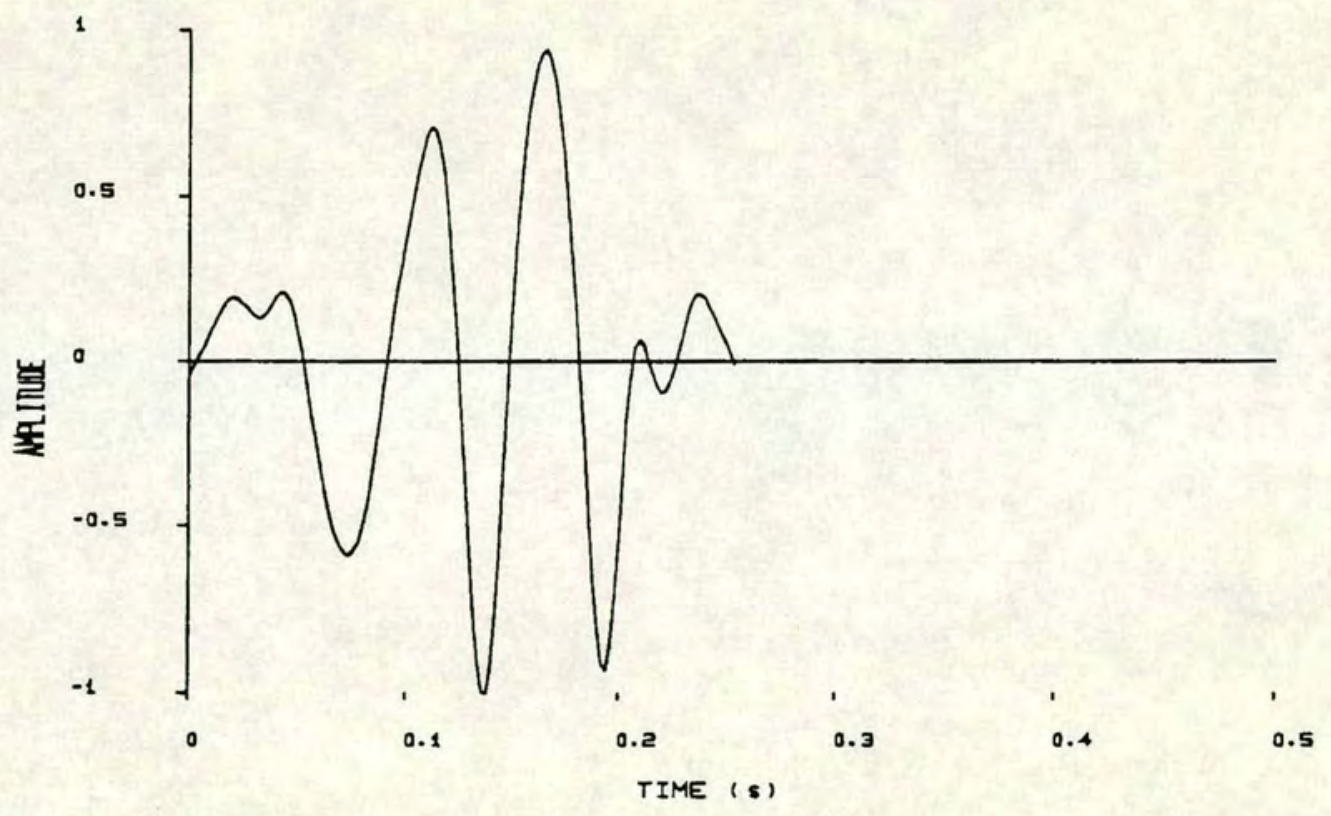

Figure 3.14 Example showing the assumed impulse response of fetal and maternal abdominal structures. This trace is obtained by averaging 10 consecutive first heart pulse waves. 


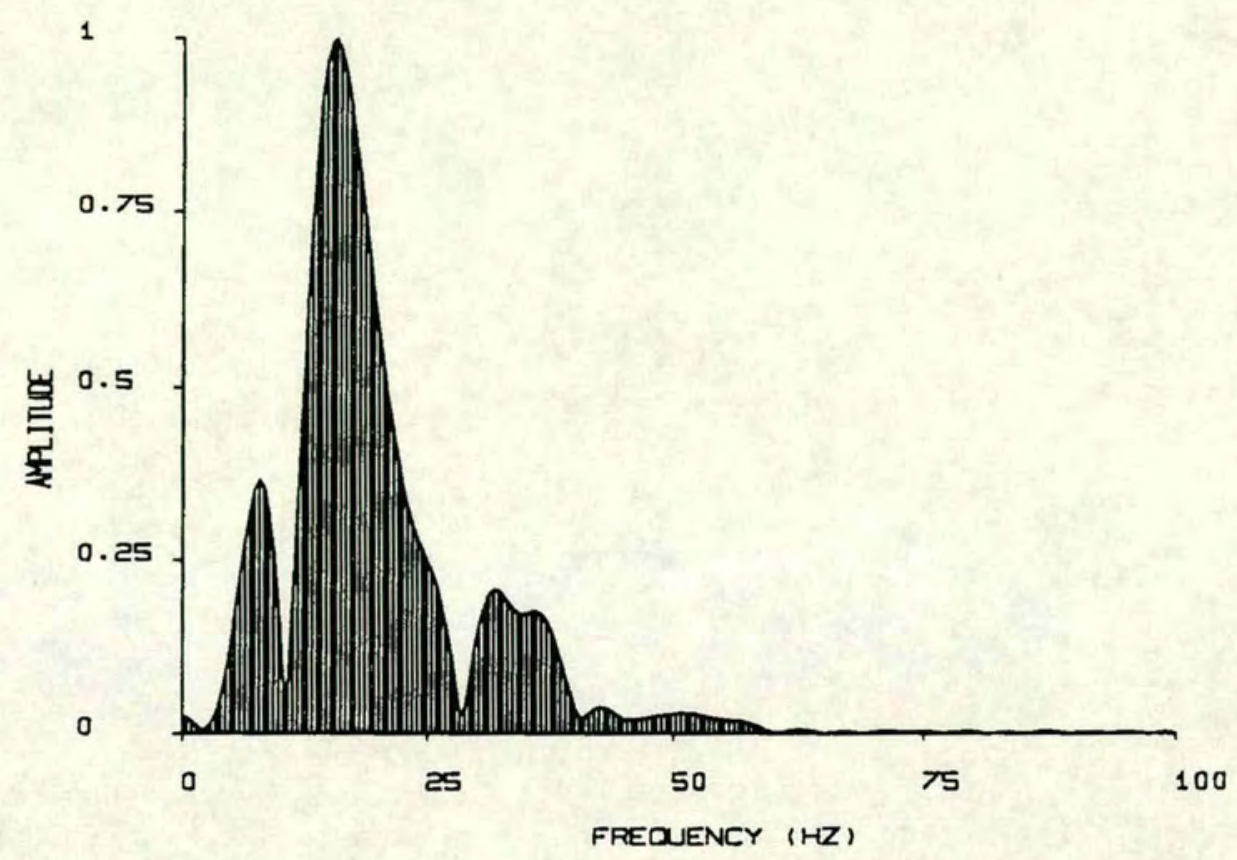

Figure 3.15 Linear spectral magnitude response of the assumed PIF model.

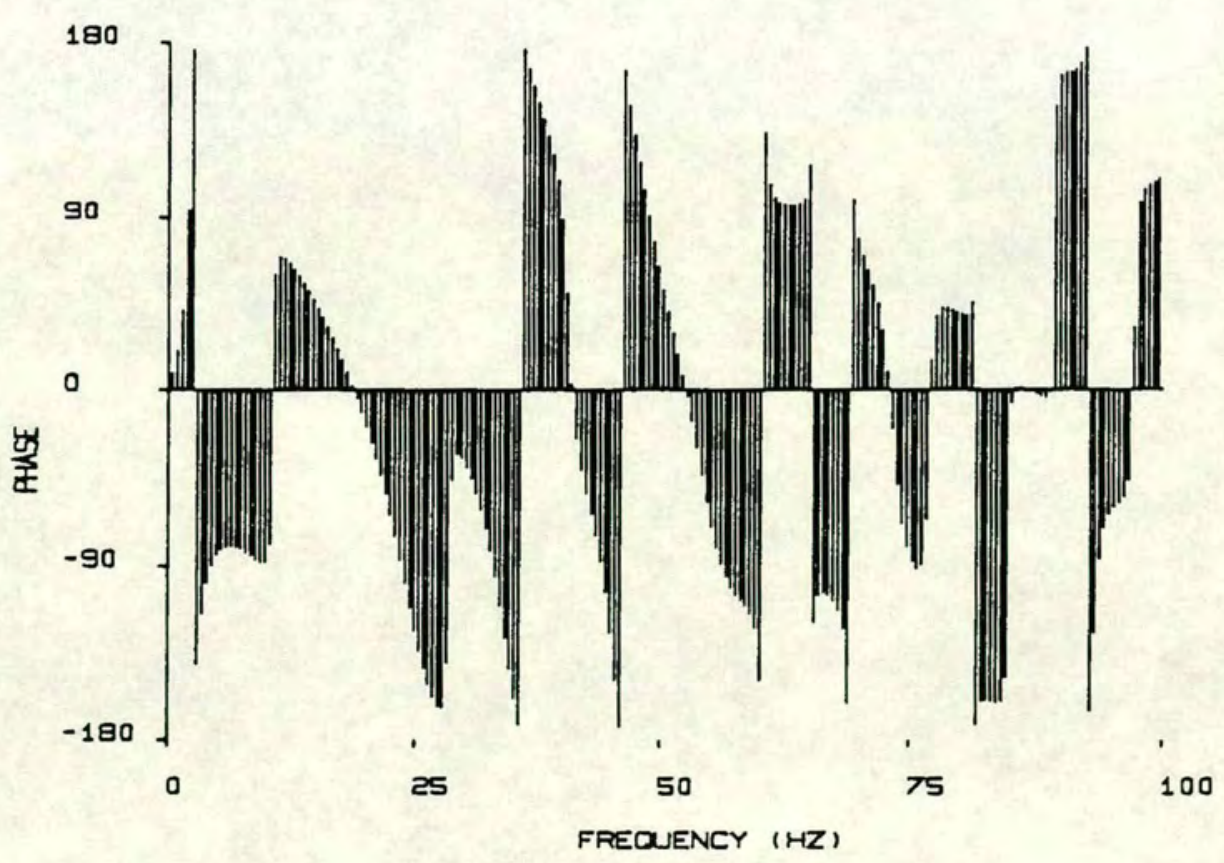

Figure 3.16 Phase response of the assumed PIF model. 
In attempting to extract FHR on a beat-to-beat basis, signal processing algorithms can utilise two main approaches, correlation filtering or inverse filtering. All methods commonly construct a template which is used for subsequent processing. In the case of correlation, the sampled time series is correlated with the obtained template weights to provide a global peak per HPW event. In the inverse filter case, the coefficients are derived using the template. This requires a computationally intensive algorithm which is sensitive to both noise and spectral shape (Least squares detailed later).

The pseudo-inverse filter algorithm presented here provides a simpler and faster approach to obtaining an approximate solution to the inversion problem. The sensitivity of true inverse filters to signal non-stationarities can also be dealt with by programming robustness into the coefficient generation algorithm. The PseudoInverse Filter algorithm is detailed:

\subsubsection{Pseudo-Inverse Filter Algorithm}

The first step in implementing this algorithm is to obtain a template of the first HPW - effectively a time averaged series (TAS) ("Template Forming" is dealt with later). The complex Fourier spectrum of this template is computed and both amplitude $|\operatorname{TAS}(\omega)|$ and phase $\operatorname{Arg}(\operatorname{TAS}(\omega))$ vectors are extracted. The first vector is then normalised with respect to the largest component thus retaining only the relative amplitudes of the constituent components. The second vector, $\operatorname{Arg}(\operatorname{TAS}(\omega))$, provides the phase relationship that exists between the spectral components of the template. For the purposes of the model adopted, this time averaged series represents an averaged impulse response of the feto-maternal organ structures, and therefore its frequency spectrum shows the associated amplitude and phase distortions (Fig. 3.15 
and 3.16). Averaging is necessary to avoid basing the model on a single HPW pulse which may contain either interfering signals (such as fetal movements) or excessive background noise.

In the case of a noiseless signal (consequently noiseless TAS template), the equalisation (deconvolution) is achieved by inverting the TAS spectrum (eq. 3.5).

$\mathrm{H}(\omega) \quad=\quad \frac{1}{\operatorname{TAS}(\omega)^{*}}$

where $H(\omega)$ is the required inverse function and TAS $(\omega)^{*}$ is the complex conjugate of the Fourier transform of the template. A classical drawback with true inverse filters (eq. 3.5) is that they are only optimal in the complete absence of additive noise (where they are the Weiner optimum solution). A further limitation which is relevant to HPW signals, is the extreme sensitivity of inverse filters to non-stationarities involving low amplitude frequency components.

A compromise is proposed for the pseudo-inverse filter which takes into account inverse filtering, emphasis of background additive noise and mild signal nonstationarity. This is achieved by setting a threshold limit on the inverted spectrum of the template: Any amplitude falling below this predetermined threshold is not inverted, in fact it is suppressed even further. In order to avoid consequent large discontinuities in the pseudo inverse spectrum, a soft threshold transition between suppression and inversion is imposed on the transform. The resulting smooth pseudoinverse spectrum is used to obtain the weights of an equalising FIR filter by inverse Fourier transformation. The smooth transition requirement between suppression and inversion is to limit the number of filter taps. A suitable function for a smooth transition over the decision boundary is the sigmoid function $\operatorname{Sig}()$ (eq. 3.6). The corresponding transfer function is shown in Figure 3.17. Before applying the sigmoid 
function, the TAS $(\omega)$ elements are normalised with respect to the largest spectral component.

$\operatorname{Sig}(x)=\frac{1.0}{1.0+e^{-x}}$

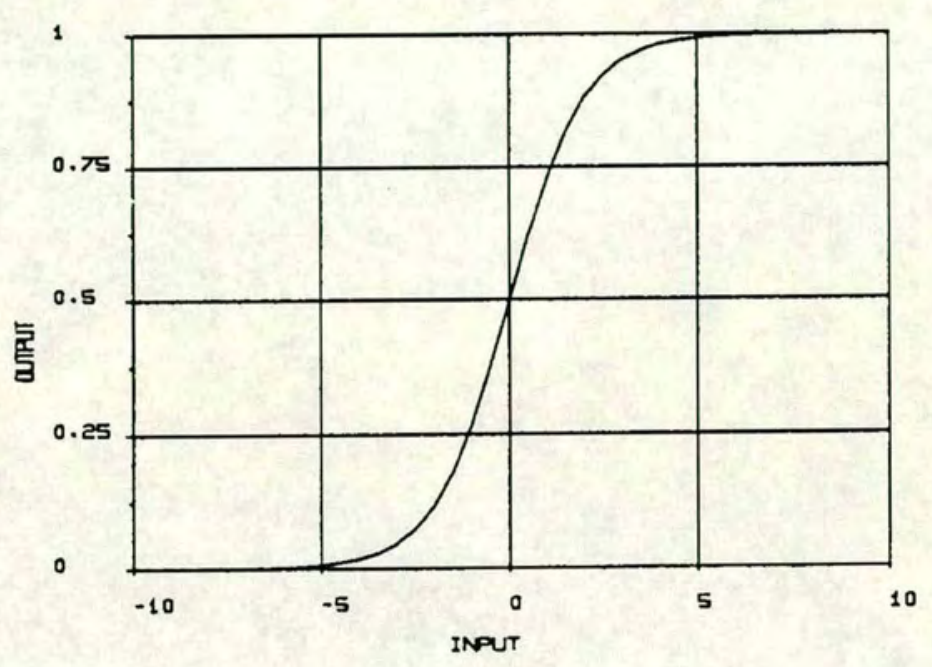

Figure 3.17 Sigmoid function mapping.

The equation of spectral pseudo-inverse mapping is given:

$H(\omega)=\frac{\operatorname{Arg}\left(\operatorname{TAS}(\omega)^{*}\right) \cdot \operatorname{Sig}(\mathrm{G} \cdot(|\operatorname{TAS}(\omega)|-\mathrm{T}))}{|\operatorname{TAS}(\omega)|+d}$

where,

$\operatorname{Arg}():$ Argument function applied to the conjugate of TAS $(\omega)$;

Sig(): Sigmoid function employed to provide a smooth cut-off transition;

$\mathrm{T}$ : Threshold determining the position of the transition between inversion and suppression, it has a range of $[0 . .1]$; 
G: Gain factor determining the slope (softness) of the sigmoid function, its useful range is [10 .. 90];

d: Small constant used to stabilise the denominator, typically $d=0.05$
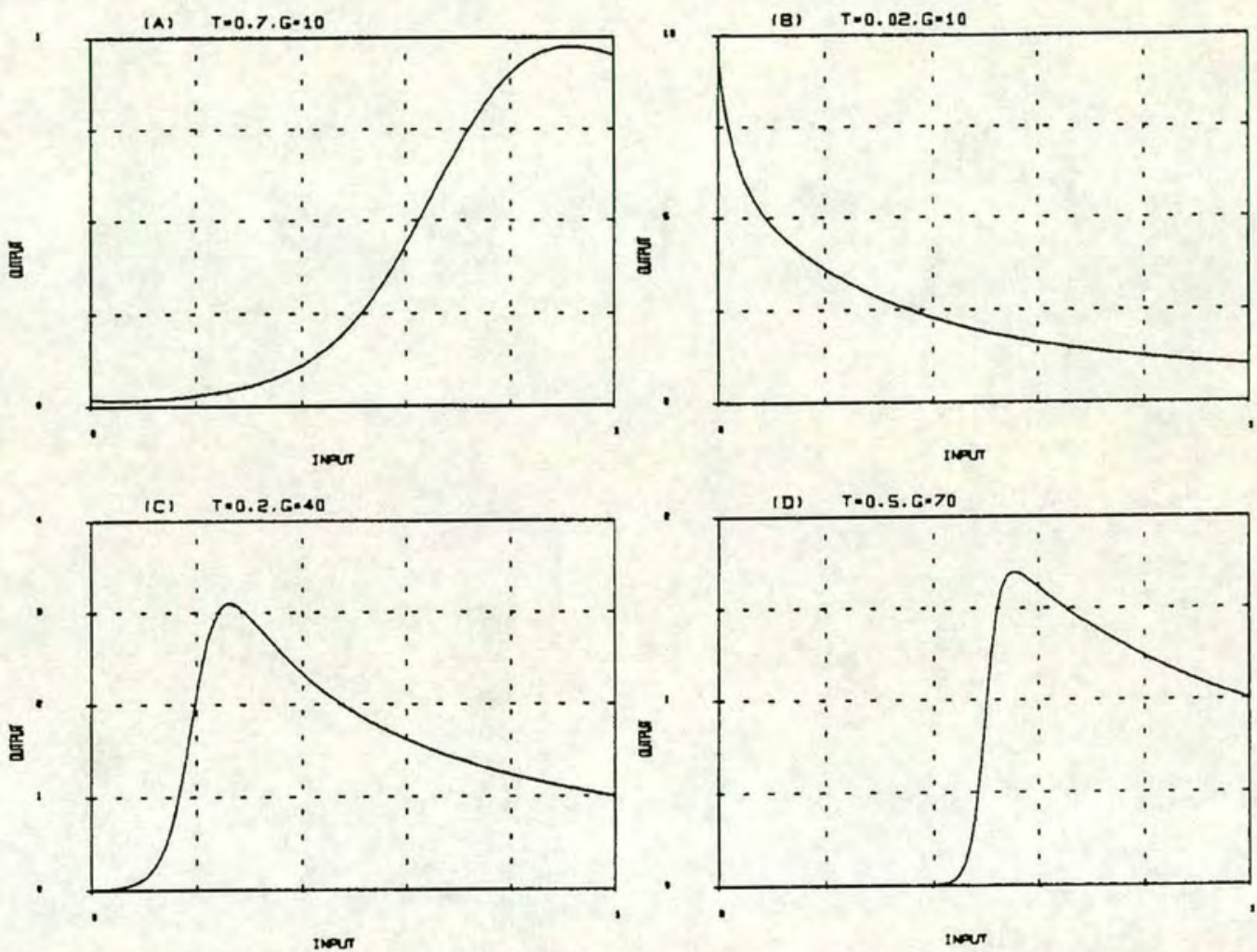

Figure 3.18 Typical magnitude mappings (eq. 3.7), $d$ is set to 0.05. $X$ and $Y$ axis are the input and output magnitudes respectively for the different $G$ and $\mathrm{T}$ parameter settings. The input amplitudes are normalised (range $[0 . .1])$, and the $\mathrm{Y}$ axis have different scales.

Figure 3.18 illustrates four examples showing the effect of changing the $T$ and $G$ parameters on the magnitude mapping (eq. 3.7). For a medium value of $T$ and a small value of $\mathrm{G}$, for example 0.7 and 10 respectively (fig. 3.18.A ), $|\mathrm{H}(\omega)|$ approximates a correlator (which results from a 1:1 magnitude mapping slope). For small values of $T$ and of $G(0.02$ and 10 respectively, fig. 3.18.B) the mapping leads to an 
approximation to a true inverse filter. However, the above two examples are extreme cases, and normally a reasonably low threshold is selected $(\mathrm{T}=0.2)$ and a mid-range gain factor $(\mathrm{G}=40)$ (Fig. 3.18.C). This provides a mapping which effectively inverts all spectral amplitudes of the TAS template lying between 0.2 and 1 , while spectral components with amplitudes lower than 0.2 are suppressed.

In cases of large interference noise components, the threshold $\mathrm{T}$ is raised accordingly (example: Fig. 3.18.D), although it will be shown in the following chapter that signals with high levels of noise are ultimately unusable for diagnostic purposes.

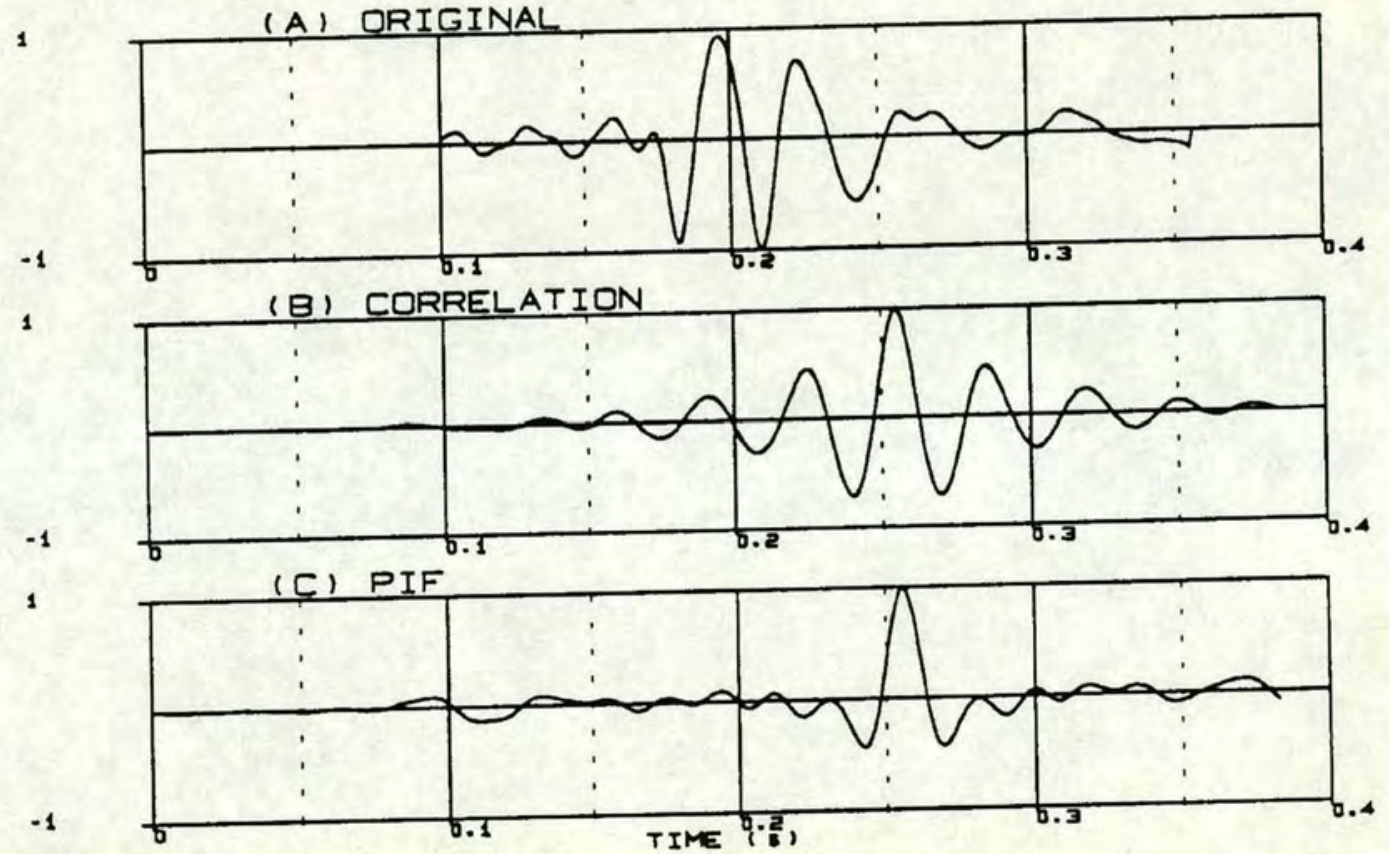

Figure 3.19 (A) Example of HPW pulse.

(B) Pulse correlation.

(C) Pseudo-inverse filter is used to provide partial equalisation.

Recall that $\mathrm{H}(\omega)$ is the magnitude and phase of the desired (equalising) filter. Hence computing the inverse Fourier transform of $\mathrm{H}(\omega)$ provides the impulse response of 
the above filter (also the weights of its transversal filter realisation). These weights can now be convolved with the sampled HPW time series thus providing a partially equalised pulse train denoting the onset of the systolic intervals. Figure 3.19.C shows the PIF equalisation output in response to a single instance of noiseless first HPW signal. Some oscillations are still evident in the PIF equalised pulse (fig. 3.19.C). These are due to the partial equalisation of the signal spectrum. However, the PIF discrimination factor, in this case, is $8: 1$ compared to $2: 1$ for the corresponding correlator output (fig. 3.19.B). The discrimination factor is defined here as the peak impulse amplitude over the peak sidelobe amplitude.

The PIF equalised spectrum illustrated in figure 3.20.B, shows the smooth transition from equalised to suppressed regions of the spectrum. The spectrum of the equalised pulse (fig. 3.20.C) has linear phase characteristics. 

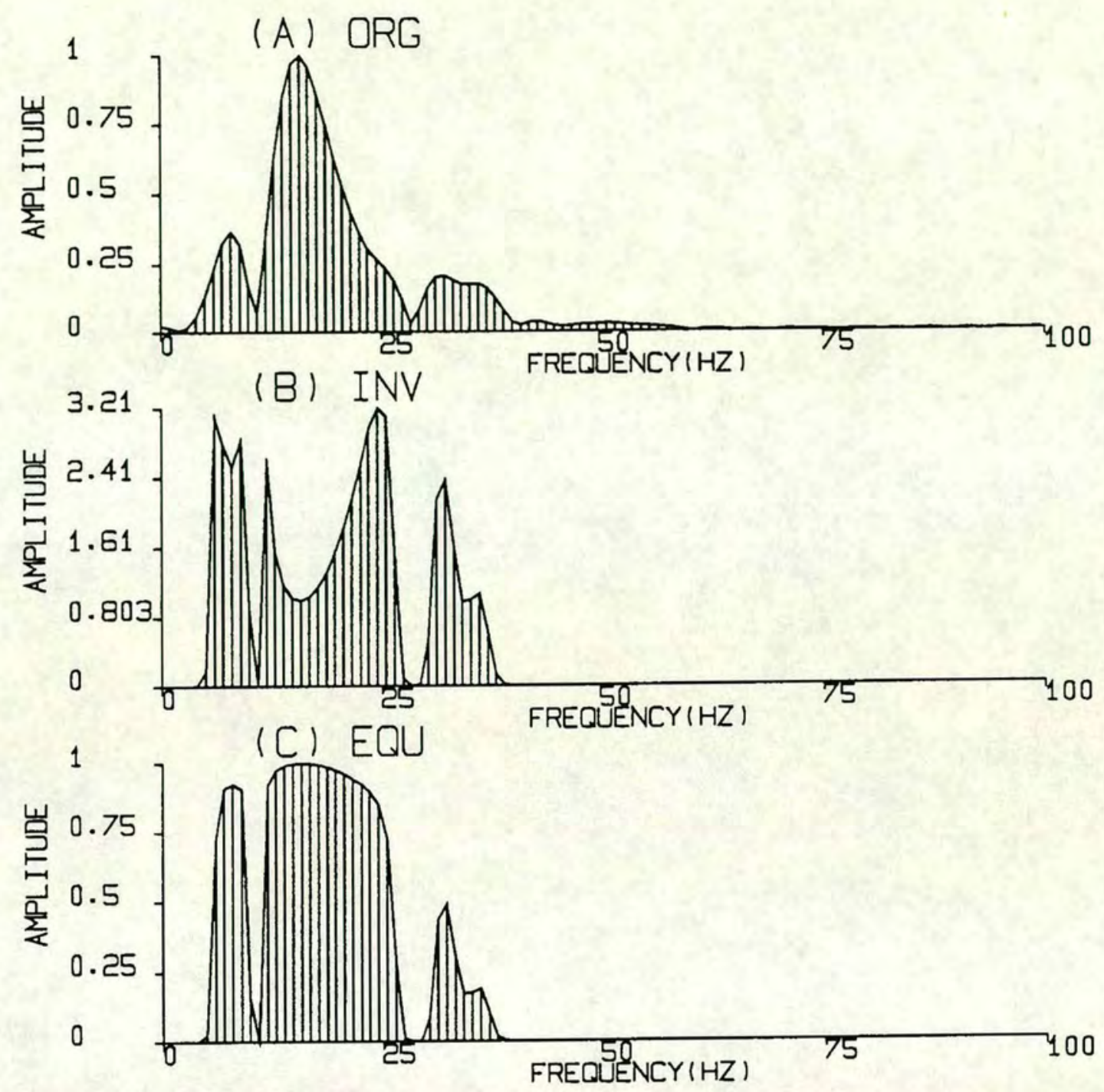

Figure 3.20 Top (A): Linear spectrum of the template; TAS $(\omega)$

Middle (B): The pseudo-inverse spectrum; $\mathrm{H}(\omega)$

Bottom (C): Spectrum of partially equalised pulse.

Conflicting requirements of fully inverting the sampled HPW's, suppressing the interfering noise and limiting the number of PIF filter coefficients influence the setting of the $\mathrm{T}$ and $\mathrm{G}$ parameters. From experience of using the PIF algorithm, it was found that a gain factor $\mathrm{G}=40$ provides a reasonably smooth transition from suppression to equalisation (hence limiting the spread of the PIF coefficients). It was 
also found that a threshold setting of $\mathrm{T}=0.2$ was large enough to take care of short term signal non-stationarities while leaving sufficient signal spectrum for equalisation therefore affording a better equalised impulse shape for a detection algorithm.

Applied to real sampled fetal HPW time series (fig. 3.21.A), the PIF equalisation technique demonstrates its capabilities in extracting the onset of systolic interval (fig. 3.21.B). A time delay due to the filtering is systematic and can be accounted for. The results obtained by correlation using the same TAS template are also shown (fig. 3.21.C). 


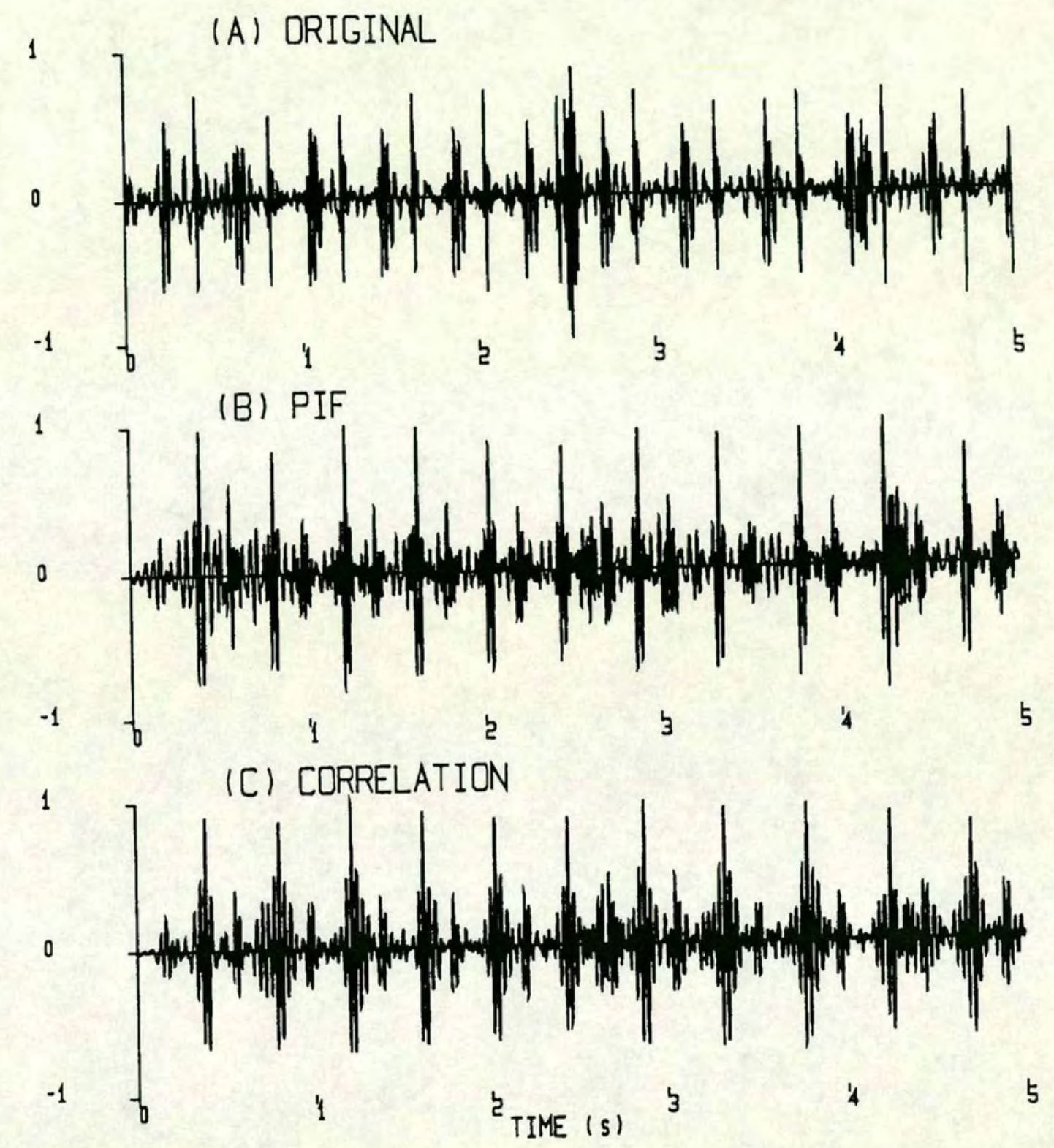

Figure 3.21 A real sampled fetal HPW signal (top trace), PIF equalisation of the first HPW (middle trace) and, correlation of the first HPW template (bottom trace). 


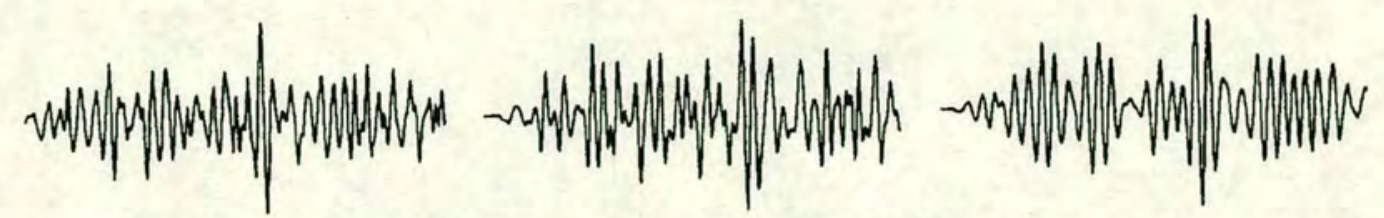

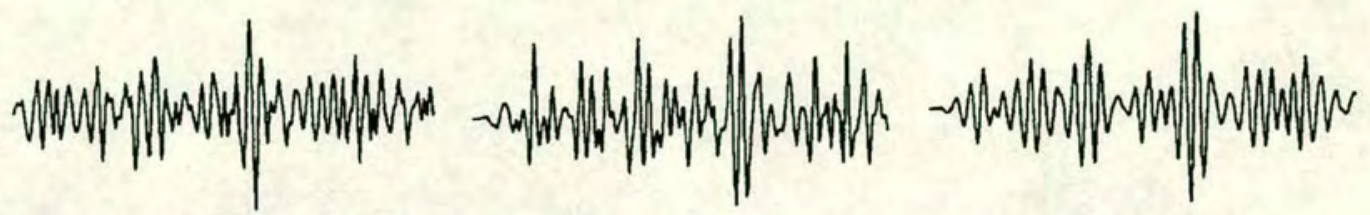

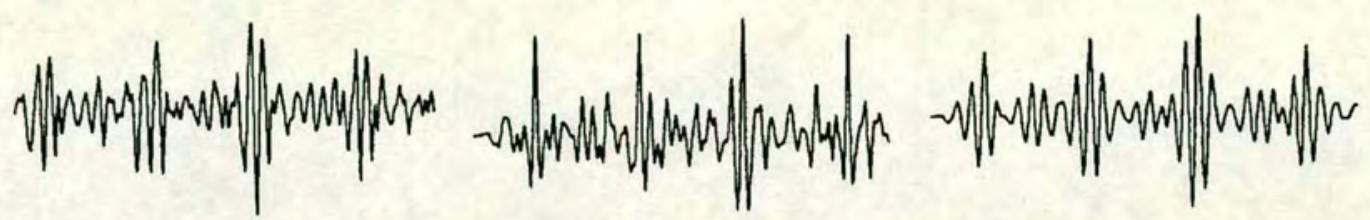

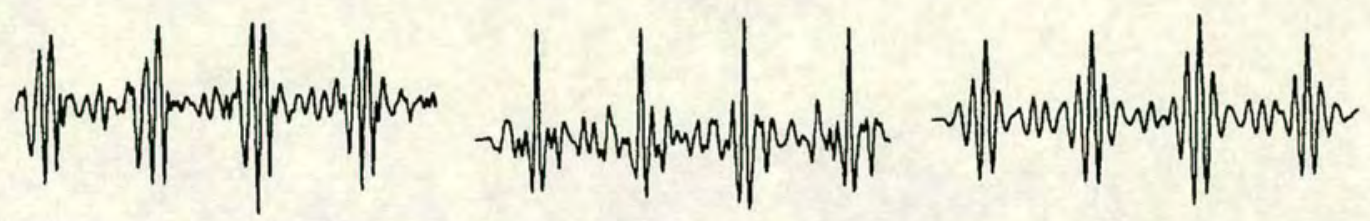

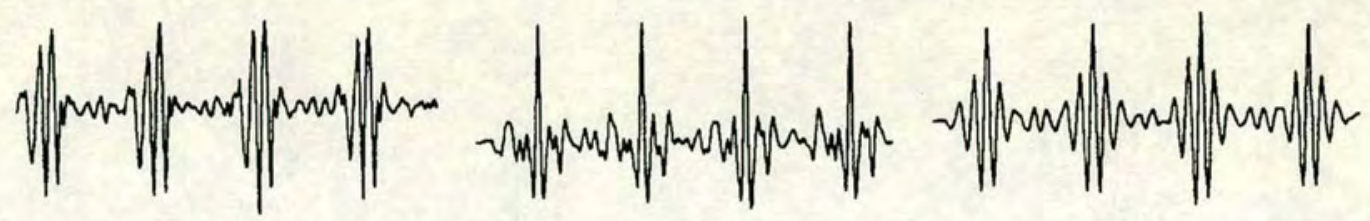

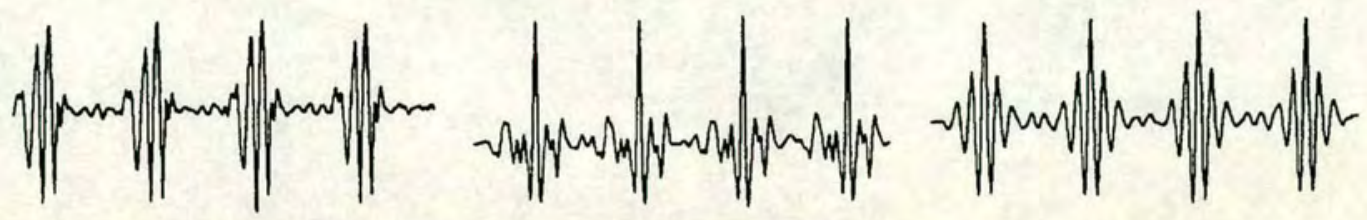

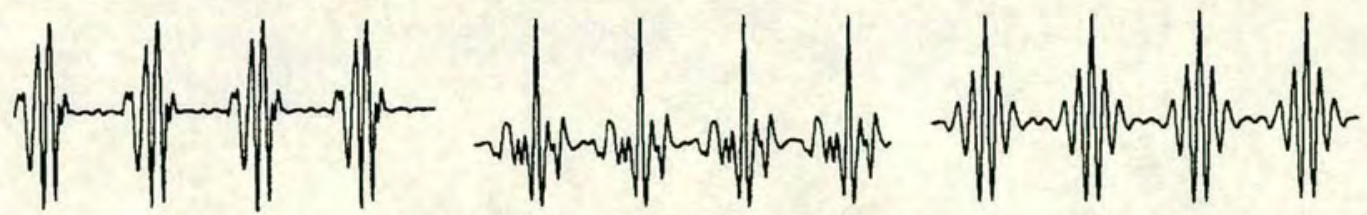

Figure 3.22 Simulated first HPW time series with increasing additive noise from bottom to top, the unprocessed trace is shown on the left side. The traces in the centre and on the right result from PIF and correlation processing respectively. 
In order to justify the PIF equalisation approach, a pictorial subjective comparison with the correlation algorithm is included. This comparison uses a simulated HPW signal vector to which noise is added at progressively higher levels. Only a limited amount of pictorial examples can be shown, and therefore performance conclusions may not be drawn. A more formal simulation for the performance assessment of estimation algorithms is provided in chapter four. It is clear however from figure 3.22 that PIF provides single, prominent, local peaks for the detection of systole events. Correlation on the other hand, while improving the SNR, results in pulse spreading (ringing) when the TAS template is oscillatory in nature (which is normally the case). 


\section{REFERENCES}

1 Abbit, P. L. "Ultrasound. A Pattern Approach", McGraw-Hill Inc. 1994. ISBN 007000031 X.

2 Wells, P. N. T. "The safety of Diagnostic Ultrasound", British Journal of Radiology, Supplement No. 20. The British Institute of Radiology, London, 1987. ISBN 090474914 6. ISSN 00071285

3 Greenhalgh, R. M. "Vascular Imaging for Surgeons", W. B. Saunders Company Ltd, 1995. ISBN 0 $70202105 \mathrm{X}$.

4 Krass, S.; Brennecke, R.; Voigtlaender, T.; Staehr, P.; Rupprecht, H. J. and Meyer, J., "Assessment of Image Quality of Intracoronary Ultrasound Systems with Tissue-Equivalent Vessel Phantoms", Computers in Cardiology, 1994. p289-92. ISBN 081866570 X.

5 Morley, P.; Donald, G. and Sanders, R. "Ultrasonic sectional anatomy", Churchill Livingstone, 1983. ISBN 0443016909.

6 Hickey, J. and Goldberg, F. "Ultrasound review of Obstetrics and Gynecology", Lippincott-Raven, 1996. ISBN 0397516541.

7 Hobbins, J. C. "Diagnostic Ultrasound in Obstetrics", Churchill Livingstone, 1979. ISBN 04430 80550 .

8 Nyberg, D. A.; Mahony, B. S. and Pretorius, D. H. "Diagnostic Ultrasound of Fetal Anomalies", Mosby-Year Book Inc., 1990. ISBN 0815164394.

9 Schmailzl, K. J. G. and Ormerod, O. "Ultrasound in Cardiology", English translation by O'Neal Wandrey, S. Blackwell Science, 1994. ISBN 0865428026.

10 Dubbins, P. A. and Joseph, A. E. A. "Ultrasound in Gastroenterology", Churchill Livingstone Inc. 1994. ISBN 0443089051.

11 Monaghan, M. J. "Practical Echocardiography and Doppler", John Wiley \& Sons, 1990. ISBN 0 $47192069 \mathrm{X}$.

12 Cavaye, D. M. and White, R. A. "A Text and Atlas of Arterial Imaging: Modern and Developing Technology", Chapman and Hall Medical, 1993. ISBN 0412461501.

13 Cavaye, D. M. and White, R. A. "Intravascular Ultrasound Imaging”, Raven Press Ltd., 1993. ISBN 0881679712 .

14 Steinke, W.; Rautenberg, W. and Hennerici, M. "Cerebrovascular Ultrasonography”, p97-127 in "Vascular Diagnostics, Noninvasive and Invasive Techniques, Periinterventional Evaluations" edited by Lanzer, P. and Rösch J. Springer-Verlag 1994. ISBN 3540579397 and 0387579397.

15 Bendick, P. J. "Correlation of Ultrasonic Image Features with Histologic Characteristics of Atherosclerotic Plaque", Proceedings of IEEE Ultrasonics Symposium, 1990. vol.3, p1357-8. 
16 The Scottish Home and Health Department and Scottish Health Service Advisory Council, "Report on Vascular Surgery Services in Scotland", Her Majesty's Stationery Office, Edinburgh, 1994. ISBN 0114952116.

17 Krass, S.; Brennecke, R.; Vorgtlaender, T.; Kuprat, R.; Staehr, P.; Rupprecht, H. J.; Fisch, A.; Darius, H. and Meyer, J. "Tissue Classification by Texture and Spectral Analysis of Intracoronary Ultrasound Radio Frequency Data", Computers in Cardiology, 1995. p641-3. ISBN 0 780330536.

18 Zheng, Y.; Foley, D. A.; Kinter, T. M. and Greenleaf, J. F. "An automated multispectral pixel and image classification system using texture analysis and neural networks", IEEE Ultrasonics Symposium, 1992, vol.2, p1093-6. ISBN 0780305620.

19 Muzzolini, R.; Yang, Y. H. and Pierson, R. "A framework for the evaluation of texture features with application to diagnostic ultrasound images", IEEE Nuclear Science Symposium and Medical Imaging Conference, 1991, vol. 3, p2201-4. ISBN 0780305132.

20 Chan, K. L. and McCarty, K. "Aspects of the statistical texture analysis of medical ultrasound images", IEE Colloquium on Ultrasound Instrumentation, 1990, vol. 3, p1-3.

21 Nailon, W. H. "Tissue Characterisation from Intravascular Ultrasound using Texture Analysis". A Ph.D. thesis, September 1997. The University of Edinburgh.

22 Nailon, W. H.; McLaughlin, S.; Spender T. and Ramo, M. P. "Textural Analysis of intravascular ultrasound images to determine coronary artery histo-pathology". Proceedings of the International Workshop of Medical and Biological Signal Processing, University of Plymouth Sept 1995. p151-56.

23 Sonka, M.; Liang, W.; Zhang, X.; DeJong, S.; Collins, S. M. and McKay, C. R. "Threedimensional automated segmentation of coronary wall and plaque from intravascular ultrasound pullback sequences", Computers in Cardiology, 1995. p637-40. ISBN 0780330536.

24 Gussenhoven, E. J.; van der Lugt, A.; The, S. H. K.; Li, W.; van Egmond, F. C., Pieterman, H. and van Urk, H. "Peripheral Intravascular Ultrasonography", p481-9 in "Vascular Diagnostics, Noninvasive and Invasive Techniques, Periinterventional Evaluations" edited by Lanzer, P. and Rösch J. Springer-Verlag 1994. ISBN 3540579397 and 0387579397.

25 Vandenberg, J.; Liersch, G.; Hanna, H. and Cameron, J. "Fully automated media and lumen boundary detection in intravascular ultrasound images", Proceedings of the IEEE Southwest Symposium on Image Analysis and Interpretation, 1996. p71-5. ISBN 0780332008.

26 Gronningsaeter, A.; Angelsen, B. A. J.; Heimdal, A. and Torp, H. G. "Vessel Wall Detection and Blood Noise Reduction in Intravascular Ultrasound Imaging", IEEE Transactions on Ultrasonics, Ferroelectrics and Frequency Control, May 1996, Vol. 43, Iss. 3, p359-69. ISSN 0885-3010.

27 Dhawale, P. J.; Rasheed, Q.; Griffin, N.; Wilson, D. L. and Hodgson, J. McB. "Intracoronary ultrasound plaque volume quantification", Proceedings of Computers in Cardiology, 1993. p121-4. ISBN 0818654708 .

28 Wikstrand, J. and Wendelhag, I. "Quantitative Ultrasonography of Carotid and Femoral Arteries", p129-38 in "Vascular Diagnostics, Noninvasive and Invasive Techniques, Periinterventional Evaluations" edited by Lanzer, P. and Rösch J. Springer-Verlag 1994. ISBN 3540579397 and 0 387579397.

29 McDicken, W. N. "Diagnostic Ultrasonics. Principles and use of instruments", Churchill Livingston, $19913^{\text {rd }}$ edition, ISBN 0443041326. 
30 Hausmann, D.; Fitzgerald, P. J. and Yock. P. G. "Coronary Intravascular Ultrasonography", p465-79 in "Vascular Diagnostics, Noninvasive and Invasive Techniques, Periinterventional Evaluations" edited by Lanzer, P. and Rösch J. Springer-Verlag 1994. ISBN 3540579397 and 0 387579397.

31 Ryan, L. K.; Lockwood, G. R.; Starkoski, B. G.; Holdsworth, D. W.; Rickey, D. W.; Drangova, M.; Fenster, A. and Foster, F. S. "A high frequency intravascular ultrasound imaging system for investigation of vessel wall properties", IEEE Ultrasonics Symposium, 1992, vol. 2, p11015. ISBN 0780305620 .

32 Freear, S.; Hoyle, B. S. and Bailey, N. J. "An Intravascular Ultrasound Imaging System", IEE Colloquium on Multiprocessor DSP (Digital Signal Processing) - Applications, Algorithms and Architectures, 1995, vol. 1, p1-5.

33 Benkeser, P. J.; Churchwell, A. L.; Lee, C. and Abouelnasr D. M. "Resolution Limitations in Intravascular Ultrasound Imaging". Journal of the American Society of Echocardiography, 1993. Vol. 6 p $158-65$.

34 Helbing, M. and Orglmeister, R. "Anisotropic filtering for detecting left ventricular borders in echocardiographic images", Proceedings of Computers in Cardiology, 1993, p197-200. ISBN 08186 54708.

35 Gronningsaeter, A.; Angelsen, B. A. J.; Gresli, A. and Torp, H. G. "Blood noise reduction in intravascular ultrasound imaging", IEEE Transactions on Ultrasonics, Ferroelectrics and Frequency Control, Vol. 42, Iss. 2, p200-9. ISSN 0885-3010.

36 Jensen, J. A. "Deconvolution of pulse-echo images", IEE Colloquium on Image Processing in Medicine, 1991, vol. 3, p1-4.

37 Belohlavek, M.; Greenleaf, J. F.; Foley, D. A. and Seward, J. B. "Utility of image enhancement methods in three-dimensional ultrasound reconstruction", Proceedings of IEEE Ultrasonics Symposium, 1991, vol. 2, p1219-22.

38 Herrington, D.; Santago, P.; Johnson, T.; Downes, T.; Braden, G. and Snyder, W. "Image processing and display of 3D intra-coronary ultrasound images", Proceedings of Computers in Cardiology, 1991. p349-52. ISBN 081862485 X.

39 Laban, M.; Oomen, J. A.; Slager, C. J.; Wentzel, J. J.; Krams, R.; Schuurbiers, J. C. H.; den Beer, A.; von Birgelen, C.; Serruys, P. W. and de Feijter, P. J. "ANGUS: a new approach to threedimensional reconstruction of coronary vessels by combined use of angiography and intravascular ultrasound", Proceedings of Computers in Cardiology, 1995, p325-8. ISBN 0780330536.

40 Evans, A. N. and Nixon, M. S. "Temporal methods for ultrasound speckle reduction", IEE Seminar on Texture analysis in radar and sonar, 1993. vol. 1, p1-6.

41 Healey, A. J. and Leeman, S. "Speckle reduction methods in ultrasound pulse-echo imaging", International Conference on Acoustic Sensing and Imaging, 1993, p68-76. ISBN 0852965753.

42 Loupas, T.; McDicken, W. N. and Allan, P. L. "An adaptive Weighted Median Filter for Speckle Suppression in Medical Ultrasound Images". IEEE Transactions of circuits and systems. Vol. 36, No. 1, Jan 1989. p129-35. ISSN 0098-4094.

43 Healey, A. J.; Forsberg, F. and Leeman, S. "Processing Techniques for speckle reduction in medical ultrasound images". IEE Colloquium on 'Image processing in medicine' 1991. Vol. 6. p1-4. 
44 Evans, A. N. and Nixon, M. S. "Speckle Filtering in Ultrasound Images for Feature Extraction", International Conference on Acoustic Sensing and Imaging, 1993. p44-9. ISBN 0852965753.

45 Migeon, B.; Serfaty, V.; Gorkani, M. and Marche, P. "An adaptive smoothing filter for URTURIP images", IEEE Engineering in Medicine and Biology Magazine, 1995, Vol. 14, Iss. 6, p762-5. ISSN. 0739-5175.

46 Schistad, A. and Taxt, T. "Speckle reduction in ultrasound images using temporal and spatial context", Proceedings of IEEE Nuclear Science Symposium and Medical Imaging Conference, 1991, vol. 3, p2210-4. ISBN 0780305132.

47 Desai, R.; Buckey, J.C. and Pearce, J. A. "An orthogonal feature preserving filter", Proceedings of IEEE International Symposium on Circuits and Systems, 1993, vol. 1, p814-7. ISBN 078031281 3.

48 Wickline, S. A.; Miller, J. G.; Recchia, D.; Sharkey, A. M.; Bridal, S. L. and Christy, D. H. "Beyond intravascular imaging: quantitative ultrasonic tissue characterization of vascular pathology", IEEE Ultrasonics Symposium, 1994, vol.3, p1589-97. ISBN 0780320123.

49 Vandenberg, J.; Campbell, D. and Cameron, J. "Arterial imaging techniques and tissue characterisation using fuzzy logic", Proceedings of the 1994 Second Australian and New Zealand Conference on Intelligent Information Systems, p239-43. ISBN 0780324048.

50 Muzzolini, R.; Yang, Y. H. and Pierson, R. "A multiresolution texture segmentation approach with application to diagnostic ultrasound images", Conference Record of the 1991 IEEE Nuclear Science Symposium and Medical Imaging Conference, vol. 3, p2205-9. ISBN 0780305132.

51 Muzzolini, R.; Yang, Y. H. and Pierson, R. "Multiresolution texture segmentation with application to diagnostic ultrasound images", IEEE Transactions on Medical Imaging, March 1993, vol. 12, Iss. 1, p108-23. ISSN 0278-0062.

52 Akiyama, I.; Saito, T.; Nakamura, M.; Taniguchi, N. and Itoh, K. "Tissue characterization by using fractal dimension of B-scan image", IEEE Ultrasonics Symposium Proceedings, 1990, vol. 3 , p1353-5.

53 Kitney, R. I.; Burrel, C.; Moura, L.; Straughan, K. and Rothman, M. T. “3D Characterization of the Arterial Wall Using Intravascular Ultrasound". IEEE Computers in Cardiology Proceedings (Cat No. 91CH3116-1) 1992, p357-60. ISBN 081862485 X.

54 Bramanti, M. "An Improved range-resolution pulse-echo technique by means of vectorialnetowrk-analyzer-based frequency-domain testing", IEEE Transactions of Ultrasonics, Ferroelectrics and Frequency Control, May 1994. Vol. 41, Iss. 3, p418-20. ISSN 0885-3010.

55 Yamada A. and Yamabe H. "Optimal time domain deconvolution for the high-resolution computerized ultrasound echo tomography", IEEE International Symposium on Acoustical Imaging, 1988; Vol. 17, p 341-50; Plenum Press New York 1989.

56 Zhu Q. and Steinberg B. "Deaberration of Incoherent Wavefront Distortion: An Approach Toward Inverse Filtering" IEEE Transactions on Ultrasonics, Ferroelectrics, and Frequency Control, May 1997; Vol. 44, p 575-89. ISSN 0885-3010.

57 Ebbini E. S.; Li, P. and Shen J. “A New SVD-Based Optimal Inverse Filter Design for Ultrasonic Applications", 1993 Ultrasonics Symposium, p 1187-90. ISSN 1051-0117.

58 Shen J.; Erdogan, H. and Ebbini E. S. "An Optimal Inverse Operator Design for Coded Excitation Ultrasound Imaging System", 1994 Ultrasonics Symposium, p 1187-90. ISSN 1051-0117. 
59 Shen J. and Ebbini E. S. "A New Coded-Excitation Ultrasound Imaging System - Part I: Basic Principles", IEEE Transactions of Ultrasonics, Ferroelectrics, and Frequency Control, Vol. 43, January 1996; p. 131-40. ISSN 08853010.

60 Shen J. and Ebbini E. S. "A New Coded-Excitation Ultrasound Imaging System - Part II: Operator design", IEEE Transactions of Ultrasonics, Ferroelectrics, and Frequency Control, Vol. 43, January 1996; p. 141-7. ISSN 08853010.

61 Shen J. and Ebbini E. S. "Real-time $3 D$ pulse-echo ultrasonic imaging with coded-excitation systems", IEEE International Conference on Image Processing, 1996; Vol. 2, p 717-720. Ceuterick B3000 Leuven, 1996. ISBN $078033258 \mathrm{X}$.

62 Nixon, M. S. "Non-invasive ultrasound 3D arterial modelling", IEE Colloquium on Medical Imaging: Image Processing and Analysis, 1992, vol. 3, p1-3.

63 Sonka, M.; Xiangmin Zhang; Siebes, M.; Bissing, M. S.; Dejong, S. C.; Collins, S. M. and McKay, C. R. "Segmentation of intravascular ultrasound images: a knowledge-based approach", IEEE Transactions on Medical Imaging, 1995, vol. 14, Iss. 4, p719-32. ISSN 0278-0062.

64 Taine, M. C.; Herment, A.; Diebold, B. and Peronneau, P. "Segmentation of cardiac and vascular ultrasound images with extension to border kinetics", Proceedings of IEEE Ultrasonics Symposium, 1994, vol. 3, p1773-6. ISBN 0780320123.

65 Meire, H. B.; Dewbury, K. C. and Cosgrove D. O. "Ultrasound teaching cases", Vol. 1 Second edition, John Wiley \& sons, 1987. ISBN 047191486 X.

66 Herrington, D. M.; Johnson, T.; Santago, P. and Snyder, W.E. "Semi-automated boundary detection for intravascular ultrasound", Proceedings of Computer in Cardiology, 1992, p103-6. ISBN 0818635525 .

67 Wenguang, L.; Bosch, J. G.; Zhong, Y.; Gussenhoven, W. J.; Rijsterborgh, H.; Reiber, J. H. C. and Bom, N. "Semiautomatic frame-to-frame tracking of the luminal border from intravascular ultrasound", Proceedings. Computers in Cardiology, 1991, p353-6. ISBN 081862485 X.

68 Bosch, J. G.; van Burken, G.; Schukking, S. S.; Wolff, R.; van de Goor, A. J. and Reiber, J. H. C. "Real-time frame-to-frame automatic contour detection on echocardiograms", Computers in Cardiology, 1994, p29-32. ISBN 081866570 X.

69 Czerwinski, R. N.; Jones, D. L. and O'Brien, W. D., Jr. "An Approach to Boundary Detection in Ultrasound Imaging”. IEEE Ultrasonics Symposium. Vol. 2, 1993. p951-5. ISBN 0780312783.

70 Czerwinski, R. N.; Jones, D. L. and O'Brien, W. D., Jr. "Edge Detection in Ultrasound Speckle Noise”. Proceedings IEEE ICIP 1994.p951-4. ISBN 0818669527.

71 Zhu, Y.; Snyder, W. E.; Braden, G. A. and Herrington, D. M. "A Gradient field metric for quality control of automated Intracoronary Ultrasound Boundaries". IEEE Computers in Cardiology 1994, p285-8. ISBN 081866570 X.

72 Steen, E. and Olstad, B. "Volume Rendering of $3 D$ Medical Ultrasound Data Using Direct Feature Mapping", IEEE Transactions of Medical Imaging Vol. 13, No. 3, Sept 1994. p517-25. ISSN 0278-0062.

73 Hokland, J. and Hausken, T. "Robust Semi-Transparent Volume Rendering of Abdominal 3D Ultrasound Data". IEEE Ultrasonics Symposium Proceedings Vol. 3, Nov 1994. p1567-71. ISBN 0 780320123. 
74 Thune, N. and Olstad, B. "Visualizing 4-D Medical Ultrasound Data", IEEE Visualization Proceedings 1991. p210-5. ISBN 0818622458.

75 State, A.; Chen D. T.; Tector, C.; Brandt, A.; Chen, H.; Ohbuchi, R.; Bajura, M. and Fuchs, H. "Case Study: Observing a Volume Rendered Foetus within a Pregnant Patient". IEEE Visualization Proceedings 1994. p364-8. ISBN 0818666277.

76 Di Mario, C.; Wenguang, L.; Linker, D. T.; de Feyter, P. J.; Bom, N.; Serruys, P. W. and Roelandt, J. R. T. C. "Three-Dimensional Intracoronary Ultrasound Goals and Practical Problems". Chapter from "Intravascular Ultrasound" edited by Roelandt, J.; Gussenhoven, E. J. and Bom, N. Kluwer Academic publishers 1993. p63-77. ISBN 0792323017.

77 Sakas, G.; Schreyer, L. and Grimm, M. "Case Study: Visualization of 3D Ultrasonic Data". IEEE Visualization Proceedings, 1994 p369-73. ISBN 0818666277.

78 Smith, S. W.; Pavy Jr., H. G. and von Ramn, O. T. "High-Speed Ultrasound Volumetric Imaging System - Part I: Transducer Design and Beam Steering". IEEE Transactions on Ultrasonics, Ferroelectrics and Frequency Control. Vol. 38, No. 2, 1991. p100-8. ISSN 0885-3010.

79 Mochizuki T. and Kasai C. "High speed acquisition scanner and three-dimensional display system using ultrasonic echo data". IEEE Ultrasonics Symposium Proceedings. Vol. 3, 1990. p 145962. Cat. No. $90 \mathrm{CH} 2938-9$.

80 Bassil, H. E. "Estimating and Processing Foetal Heart Signals from Phonocardiographic Signals", A PhD thesis, 1991. The University of Edinburgh, p62-87.

81 Scottish home and health department "Obstetric Ultrasound in Scotland", Her Majesty's Stationery Office, Edinburgh, 1988. ISBN 0114934282.

82 Janssen, J. “Clinical Application of Video Image Processing in Cardiac Angiology”, Van Gorcum, Assen, 1989. p13. ISBN 9023223926.

83 Kremkau, F. W. "Diagnostic Ultrasound, principles, instruments, and exercises", 3 rd edition, W. B. Saunders Company, 1989. ISBN 0721628230.

84 Bushong, S. C. and Archer, B. R. "Diagnostic Ultrasound, Physics, Biology, and Instrumentation”, Mosby-Year Book Inc., 1991. ISBN 0801603943.

85 Barnett, E. and Morley P. "Clinical Diagnostic Ultrasound”, Blackwell Scientific Publications, 1985. ISBN 0632008970.

86 Feigenbaum, H. "Echocardiography” fifth edition, Lea and Febiger, 1994. ISBN 0812116925.

87 Alonso, M and Finn, E. J. "Fundamental University Physics, Vol. II, Fields and Waves". AddisonWesley Publishing Company, 1967.

88 Lerski, R. A. “Practical Ultrasound”, IRL Press Limited, 1988, ISBN 1852210680.

89 Quistgaard, J. U. "Signal Acquisition and Processing in Medical Diagnostic Ultrasound", IEEE Signal Processing Magazine, January 1997 Vol. 14, No. 1, p67. ISSN 1053-5888.

90 Bijnens, B.; Herregods, M. C.; Nuyts, J.; Suetnes, P. and Van de Werf, F. "Reconstruction of Echocardiographic Sector Images from Digital Reaiofrequency Data", IEEE Computers in Cardiology, 1994, p453-6. ISBN 081866570 X. 
91 Bovik, A. "On Detecting Edges in Speckle Imagery". IEEE Transactions of acoustics, speech, and signal processing. Vol. 36, No. 10, Oct 1988. p1618-27. ISSN 0096-3518.

92 Harmuth, H. G. "Acoustic Imaging with Electronic Circuits", Academic Press, 1979.ISBN 01201 45715

93 Miller, G. "Invasive Investigation of the Heart - a guide to cardiac catheterisation and related procedures", Blackwell Scientific Publications, 1989. ISBN 063202027 X.

94 Tobis, J. M. and Yock, P. G. "Intravascuar Ultrasound Imaging”, Churchill Livingstone, $1^{\text {st }}$ ed., 1992.

95 Karaman, M.; Kutay, A. and Bozdagi G. "An Adaptive Speckle Suppression Filter for Medical Ultrasonic Imaging”. IEEE Transactions of Medical Imaging. Vol. 14, No. 2, June 1995. p 283-92. ISSN 0278-0062.

96 Li, W. "Image and Signal Processing in Intravascular Ultrasound". A Ph.D. Thesis, June 1997. The University of Rotterdam.

97 Wiener N. "Extrapolation, Interpolation and Smoothing of Stationary Time Series", NDRC report 370, February 1, 1942, published as "Extrapolation, Interpolation and Smoothing of Stationary Time Series with Engineering Applications", MIT Press Cambridge Mass., 1949.

98 Haykin S. "Adaptive Filter Theory" $2^{\text {nd }}$ Edition, Prentice-Hall International Editions. ISBN 013 0055131.

99 Guy, E. V. and Khan, R. H. "Three channel Wiener Filter for Suppression of Speckle Noise in SAR Images of Ocean Scenes", Canadian conference on Electrical Engineering and Computer Engineering, 1994, Vol. 2 p 433-6. ISBN 0780324161.

100 Leung, C. and Lu, W. S. "A Modified Wiener Filter for the Reconstruction of Blurred Images", IEEE Pacific Rim Conference on Communications Computers and Signal Processing Vol. 1, p 166-9. ISBN 0780309715.

101 Liu, Dong-Lai and Waag, Robert C. "Correction of ultrasonic wavefront distortion using backpropagation and a reference waveform method for time-shift compensation" Journal of the Acoustic Society of America, August 1994, p 649-60.

102 Anh, V. V. and Spencer N. M. "Unbiased Wiener Filtering: Nonstationary Processes", Signal Processing 1991, Vol. 25 p 51-68. ISSN 01651684.

103 Shao, L.; Lewitt, R. M.; Karp, J. S. and Contryman, P. "Practical Considerations of Wiener Filtering in PET", IEEE Transactions on Nuclear Science, Vol. 41 Issue 4, p 1560, 1994. ISSN 0018 9499.

104 Shao, L.; Lewitt, R. M.; Karp, J. S. and Muehllehner, G. "Combination of Wiener Filtering and Singular Value Decomposition Filtering for Volume Imaging PET", IEEE Transactions on Nuclear Science, Vol. 42 Issue 4, part 1, p 1228-34, 1994. ISSN 00189499.

105 Karpovsky, M. G. and Trachtenberg, E. A. "Some Optimisation Problems for Convolution Systems Over Finite Groups", Information and Control, Vol. 34, p 227-47, 1977.

106 Trachtenberg, E. A. and Karpovsky, M. G. "Filtering in a Communication channel by a Fourier Transform Over Finite Groups", in "Spectral Technologies and fault detection" edited by Karpovsky, M. G., Orlando: Academic Press, 1985. 
107 Xin, J. and Bilgutay, N. M. "Ultrasonic range resolution enhancement using L1 norm deconvolution”, 1993 Ultrasonics Symposium, Vol. 2, p 711-4. ISBN 0780312783.

108 Bassil, H. E.; Dripps, J. H. and Boddy, K. “Aspects of Phonocardiographic Fetal Heart Monitoring". 2nd Portuguese Congress on Biomedical Engineering, 1990. p10 4 1-10 45.

109 Bassil, H. E. and Dripps, J. H. "Real Time Processing and Analysis of Fetal Phonocardiographic Signals", Clinical Phys Physiol Measurements, 10 Suppl B, 1989. p67-74.

110 Young, W. F. and McLaughlin, S. "Pseudo Inverse Filter Design for improving the Axial Resolution of Ultrasound Images", IEEE Tencon Conference on Speech and Image Technologies for Computing and Telecommunications, 1997, Vol. 2, p703-6. ISBN 0780343654.

111 Nailon, W. N.; McLaughlin, S.; Spencer, T. and Ramo, M. P. "Intravascular Ultrasound Image Interpretation", Proceedings $13^{\text {th }}$ International Conference on Pattern Recognition, IEEE Computer Society, Vol. C, p503-7, 1996. ISBN 0818672765.

112 Delacharte, P.; Tray, D.; Zhigang, S.; Gimenez, G. and Dziedzic, A. "Estimation of the causal impulse response of underwater target", IEEE International Conference of Acoustics, Speech and Signal Processing (ICASSP), 1994. Vol. 2, p385-8. ISBN 0780317750. 